\title{
Blast Hazard Mitigation through Vented Suppressive Shields
}

\author{
by \\ OMAR ABDELALIM \\ A thesis submitted to the Faculty of Graduate and Postdoctoral Affairs in partial \\ fulfillment of the requirements for the degree of \\ DOCTOR OF PHILOSOPHY \\ in \\ Civil and Environmental Engineering Department \\ Carleton University \\ Ottawa-Carleton Institute of Civil and Environmental Engineering \\ Ottawa, Ontario \\ (C) Copyright \\ 2013, Omar Abdelalim
}




\section{Abstract}

Vented Suppressive shield (VSS) containers have traditionally been used for the storage of hazardous materials, especially explosives. The role of VSS containers is to attenuate the blast pressure and impulse outside the container and to eliminate primary fragment hazard associated with accidental explosions. Most VSS containers are typically designed from experience and observations of previous container testing programs or lessons learned from previous accidents. Another method that is used to analyze and design VSS structures is using computational fluid dynamic (CFD) software packages such as AUTODYN that are expensive, has long computational time as well as requires special expertise to get the best use of it.

The aim of this study is to develop a reliable design methodology that may be used to design the elements of the VSS containers without the need of using CFD software packages. The study begins with defining the vent area ratio for the studied VSS sections. AUTODYN was used to calculate the pressure outside several VSS containers with different cases. The obtained data were utilized to develop a set of equations to predict the pressure and impulse outside the container. The pressure values obtained from the equations showed a good correlation with the results obtained from previous experimental results.

The second part of the thesis was studying the pressure profile on the elements of the VSS containers. The pressure profiles on the side wall of different VSS sections were studied using 2D AUTODYN models. Some modifications were made to the Friedlander's waveform equation in order to take into account the effect of internal explosion. The single layer plate was chosen as a control configuration because of the simplicity of its section and geometric coefficients were introduced in order to take into account the effect of different VSS geometric sections. The pressure profiles obtained from the developed equations showed a good correlation with those obtained from AUTODYN.

Finally, a single degree of freedom (SDOF) model was developed to study the structural response of the VSS elements due to the applied blast loading. The SDOF model was able to predict the structural behaviour of the steel VSS elements. The results were compared with those obtained from AUTODYN software and a good correlation was found between the two responses. 


\section{Dedication}

I would like to dedicate this thesis to my late father, Assem Abdelalim who always showed an unparalleled example of excellence every day of his life. His exemplary work in the area of engineering guided me in my academic and professional career. His wisdom, love and encouragement made me the person and engineer that I am today. I am forever grateful for the sacrifices that he made to ensure that I was successful in any path that I might choose in life. 


\section{Acknowledgments}

I would like to express my deepest gratitude to my co-supervisors Professors Braimah and Abd El Halim for their guidance, support and generous advice in completing my thesis. It was a pleasure working under their supervision for the past 5 years. I would also like to thank Professor Ettore Contestabile for serving on my advisory committee, as well as his generous advice. I must acknowledge the financial support provided by NSERC and Carleton University throughout my Ph.D. study.

I would like to thank the faculty members and staff at Carleton University who were always doing their best to offer outstanding working environment to the students. I would like also to thank my friends for their support throughout the years. Everyone I have met in Ottawa has been a great friend and colleague; specifically my dear friends Ahmed Nasser and Hasan Tayyeb for their continuous support.

I extend my appreciation to my lovely great family; especially my late father; Professor Assem Abdelalim, my mother; Azza Aboeldahab and siblings; Ali, Abdelrahman and Aliaa who have given me unlimited love, support and encouragement through the challenging times of my life.

A special thank you to my fiancée; Marwa Elkady. Words cannot describe how lucky I am to have her in my life. She has selflessly given me more than I ever could have asked for. I love you, and looking forward to our lifelong journey.

Thank you all!! 


\section{Table of Contents}

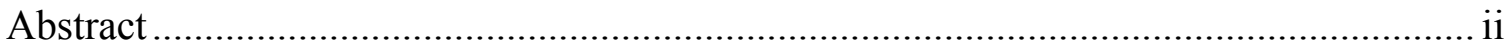

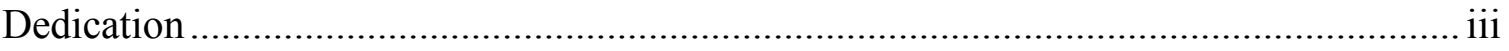

Acknowledgments.................................................................................................... iv

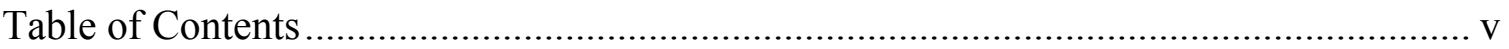

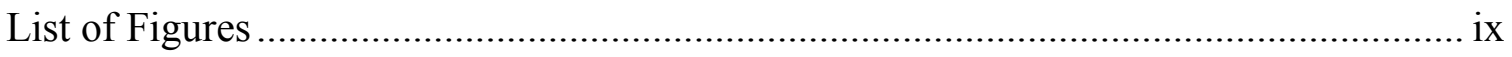

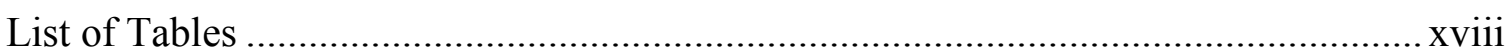

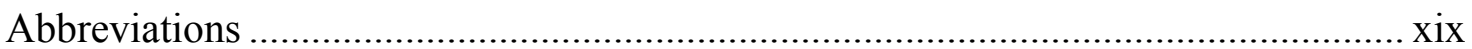

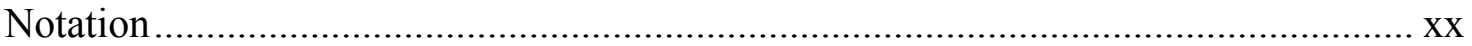

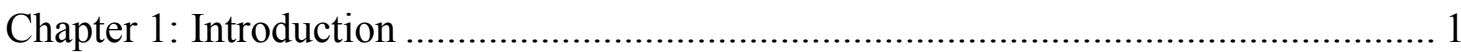

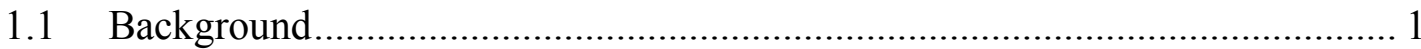

1.2 Research Goals and Approach....................................................................... 2

1.3 Thesis organization ................................................................................... 3

Chapter 2: Literature Review ……………............................................................. 5

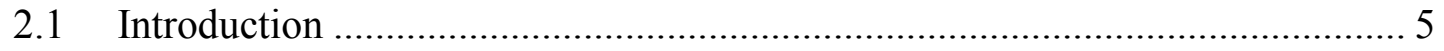

$2.2 \quad$ Classification of Explosions ........................................................................ 5

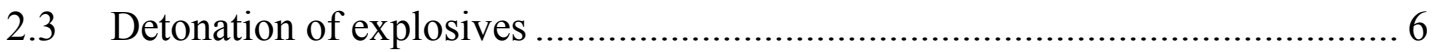

$2.4 \quad$ Reflected Blast Waves........................................................................ 7

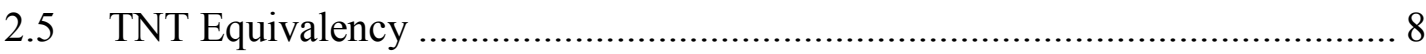

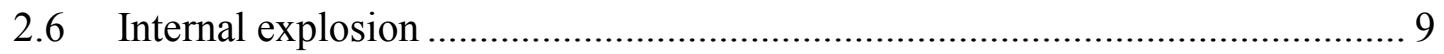

2.7 Explosion Accidents .............................................................................. 13 


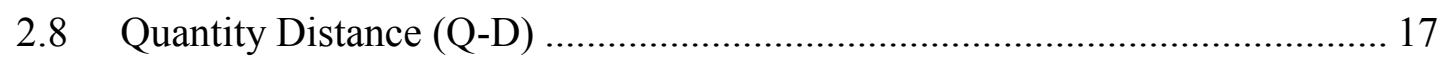

2.9 Storage and transportation of hazardous materials ........................................... 18

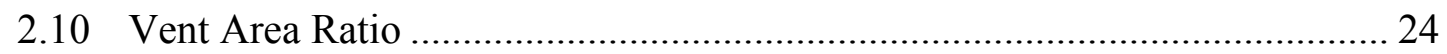

2.11 Calculation of Pressure inside enclosed structures ........................................ 26

2.12 Previous Work on Suppressive Shields ..................................................... 28

2.13 Finite Element Modelling (FEM) ............................................................. 32

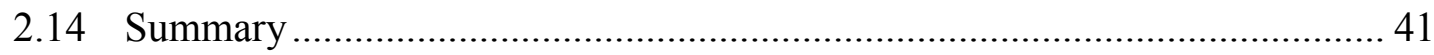

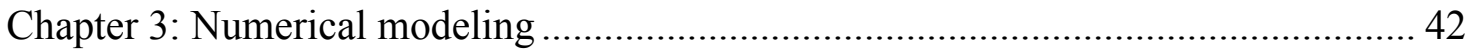

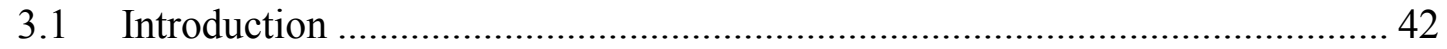

3.2 Model Description (AUTODYN 2D model) ……......................................... 42

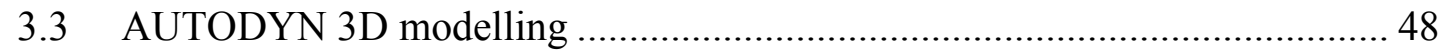

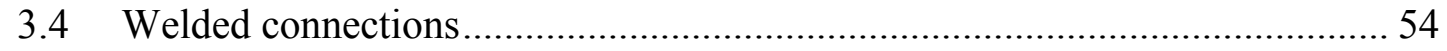

$3.5 \quad$ Summary

Chapter 4: Pressure and Impulse attenuation outside VSS containers........................... 56

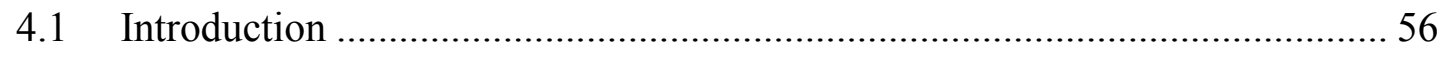

4.2 Vent area ratio for VSS geometric configurations ......................................... 56

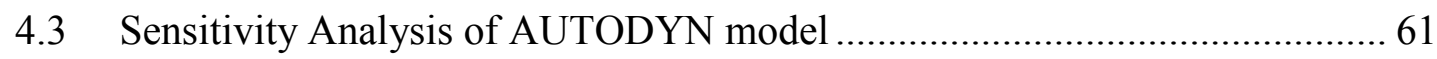

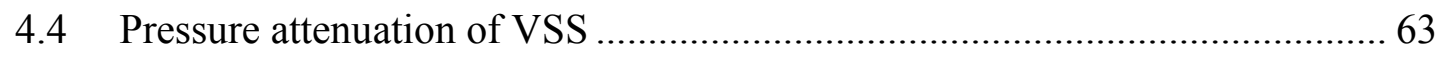

4.5 Factors affecting the pressure outside VSS containers..................................... 64

4.6 Comparison between VSS configurations ………………............................. 73

4.7 Prediction of blast pressure outside vented structures.................................... 76 
Chapter 5: Pressure on Vented Suppressive Shield elements . 85

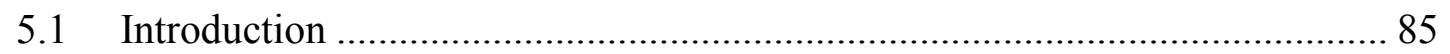

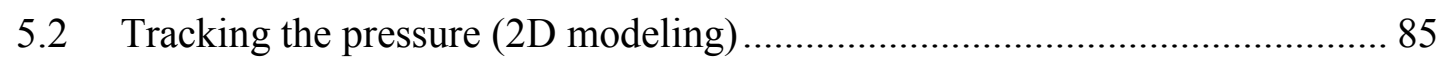

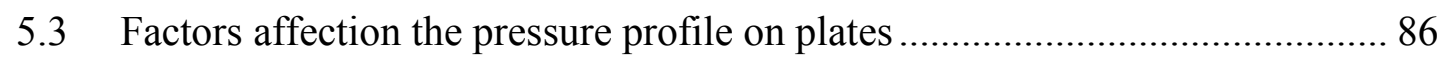

5.4 Empirical equations to calculate the pressure on the side walls of vented Structures 102

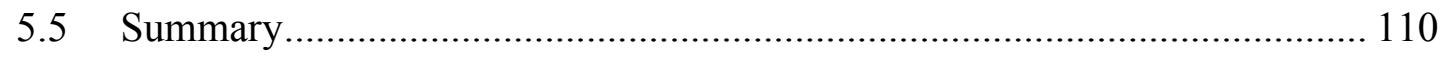

Chapter 6: Structural Design of VSS ................................................................. 112

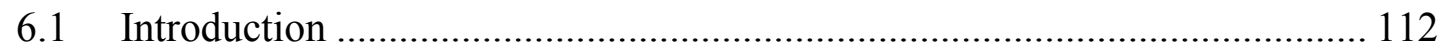

6.2 Verification of AUTODYN model ......................................................... 112

6.3 Single Degree of Freedom Method ............................................................ 114

6.4 Results obtained from SDOF model ......................................................... 129

6.5 SDOF analysis of single-layer steel angle configuration ........................... 144

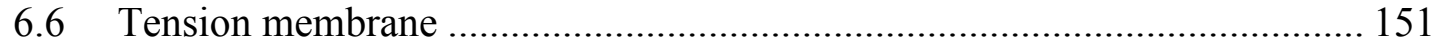

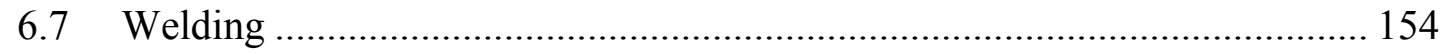

6.8 Suggested VSS sections (Single-layer steel plate) …............................. 158

6.9 Suggested VSS sections (Single-layer side-by-side angle interior-facing) ... 159

6.10 Comparison between configurations................................................ 160

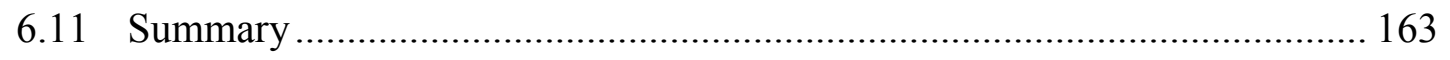

Chapter 7: Conclusions and Recommendation for Future Work ............................. 164 


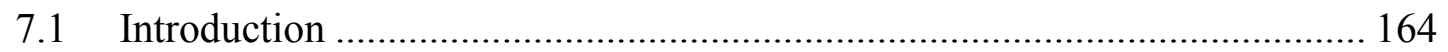

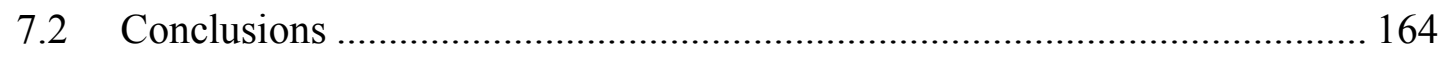

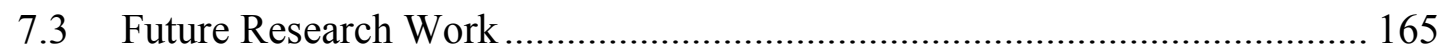

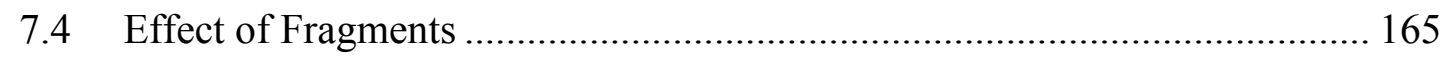

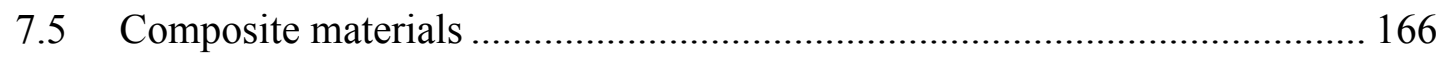

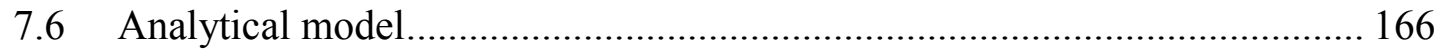

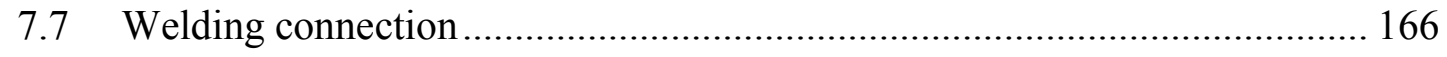

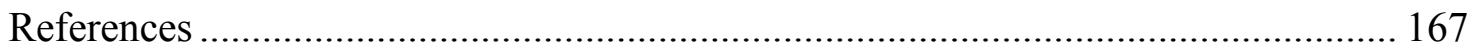

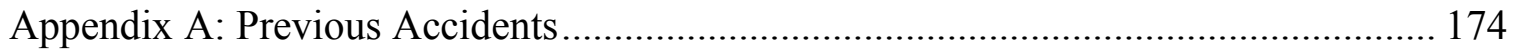

Appendix B: Computational methods..................................................................... 180

Appendix C: Results for the prediction of pressure profiles......................................... 181

Appendix D: Alogarithm for step-by-step solution for elasto-plastic single degree of

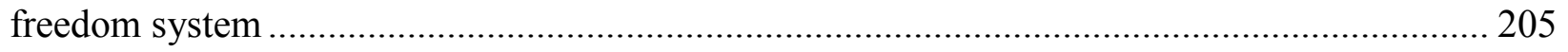




\section{List of Figures}

Figure 1: Flow chart for the research plan of the thesis........................................................... 3

Figure 2: Typical blast wave profile (Braimah, 2012) ………….............................................. 7

Figure 3: Blast wave reflection within confined structure (Braimah et al, 2012)........................ 10

Figure 4: Typical time history for internal pressure at inner surface of enclosed structures,

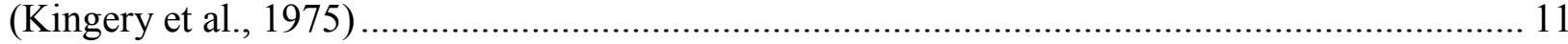

Figure 5: Combined shock and gas Pressures (square chamber), (UFC3-340-02, 2008)............. 12

Figure 6: Combined shock and gas pressures (long rectangular chamber), (UFC3-340-02, 2008)

Figure 7: Explosion at the Evangelos Florakis Naval Base in Cyprus (Demotix, 2011).............. 14

Figure 8: The damage in the bridge due to the explosion (New York Times, 2013) ................... 15

Figure 9: Damage of the body of vessel (Weeth \& Associates LLC, 2006) ................................ 16

Figure 10: Typical Shipping Container Exploded View (Ecocontainerhome website)................ 19

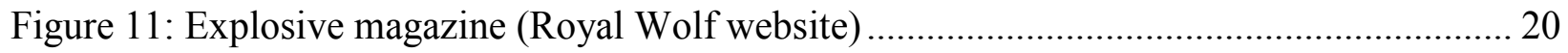

Figure 12: Perforated steel layer, (Palley et al., 2006)................................................................ 21

Figure 13: Double layer steel containments, (Palley et al., 2006) .............................................. 22

Figure 14: Vented Suppressive Shield containment, (King et. al, 1981)...................................... 23

Figure 15: Definition of Vent Area Ratios for Nested angles configuration, (Baker et al. 1982) 25

Figure 16: Definition of Vent Area Ratios for Side-by-side angles configuration, (Baker et al.

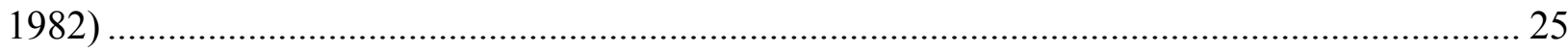

Figure 17: Simplified internal blast pressure (Baker et al., 1983) …………………………....... 28

Figure 18: Deformation in the container (Crane et al. 1997) ................................................... 30 
Figure 19: Suppressive Shield Panels studied (ElShafey, 2009) 31

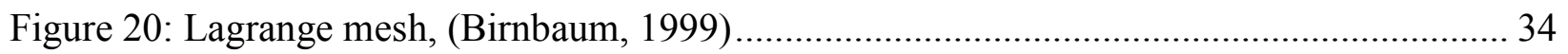

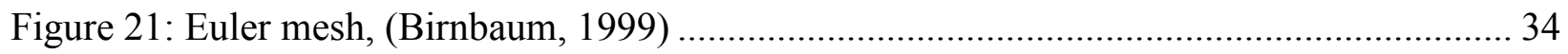

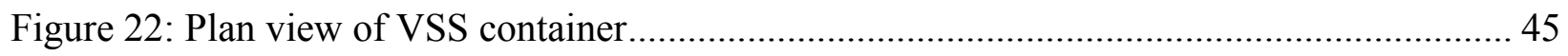

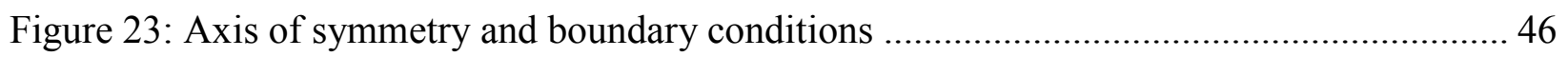

Figure 24: Location of Gauges outside the container and on the VSS elements ....................... 47

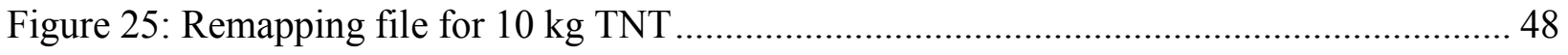

Figure 26: Example for 3D VSS container model ................................................................ 50

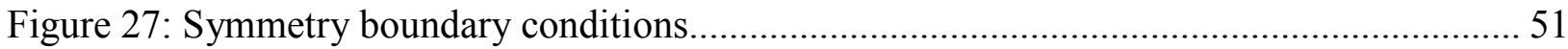

Figure 28: Fixed boundary condition at main frame ....................................................... 52

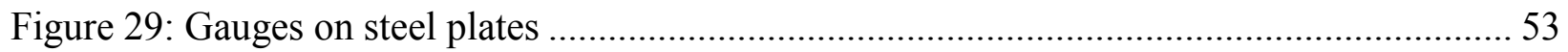

Figure 30: Connection between steel plates and the main frame ......................................... 55

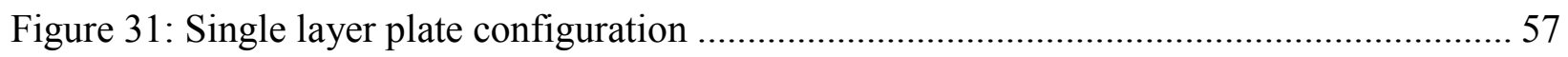

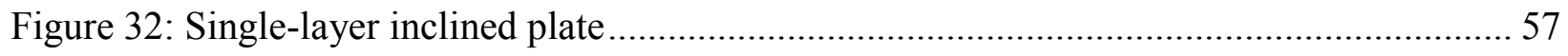

Figure 33: (a) Single-layer side-by-side angle exterior-facing, (b) Single-layer side-by-side angle

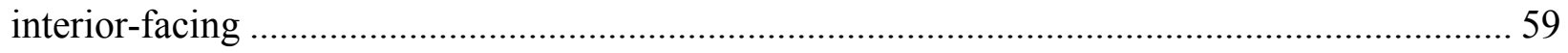

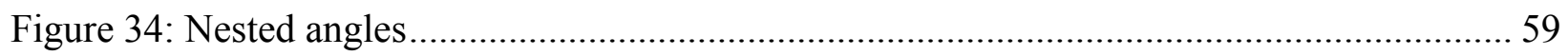

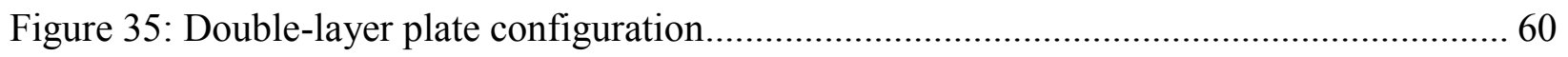

Figure 36: Double-layer nested side-by-side angle configuration (Koger et al., 1975) ............. 61

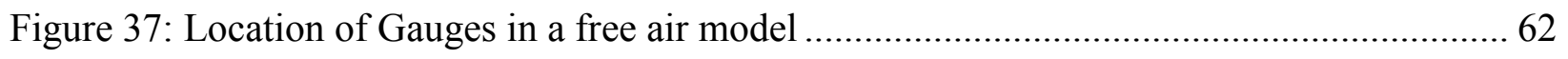

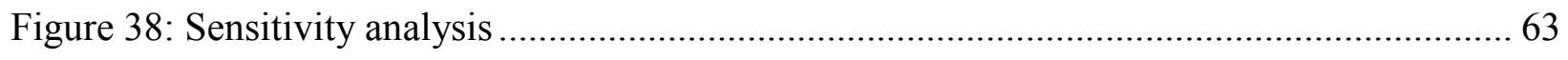


Figure 39: Velocity vectors at VSS location.......

Figure 40: Effect of vent area ratio on the pressure outside 65

Figure 41: Effect of container size on the pressure outside the container 66

Figure 42: Effect of $g / a$ ratio on peak incident pressure outside VSS container $(20 \mathrm{~kg}$ TNT charge) 68

Figure 43: Relation between Pressure values and scaled distance for VSS section with 0.16 vent area ratio $($ TNT charge $=5 \mathrm{~kg})$

Figure 44: Relation between Pressure values and scaled distance for VSS section with 0.16 vent area ratio $(\mathrm{TNT}$ charge $=10 \mathrm{~kg})$. 70

Figure 45: Relation between Pressure values and scaled distance for VSS section with 0.16 vent area ratio $(\mathrm{TNT}$ charge $=20 \mathrm{~kg})$ 70

Figure 46: Relation between Pressure values and scaled distance for VSS section with 0.20 vent area ratio $(\mathrm{TNT}$ charge $=5 \mathrm{~kg})$ 71

Figure 47: Relation between Pressure values and scaled distance for VSS section with 0.20 vent area ratio $(\mathrm{TNT}$ charge $=10 \mathrm{~kg})$. 72

Figure 48: Relation between Pressure values and scaled distance for VSS section with 0.20 vent area ratio $($ TNT charge $=20 \mathrm{~kg})$ 72

Figure 49: Pressure values for different geometric configurations with 0.25 vent ratios 75

Figure 50: Impulse values for different geometric configurations with 0.25 vent ratios 76

Figure 51: Comparison between available equations and numerical results (AUTODYN) 79

Figure 52: Comparison between obtained Pressure values from proposed equation and numerical results 81

Figure 53: Comparison between obtained Impulse values from proposed equation and numerical results 
Figure 54: Comparison between experimental pressure values and predicted pressure values ... 82

Figure 55: Comparison between experimental Impulse values and predicted Impulse values .... 84

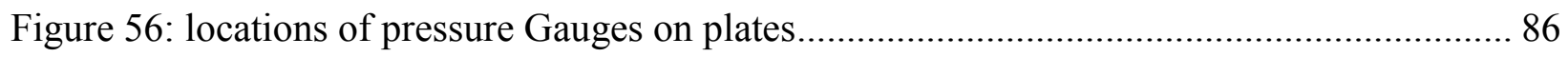

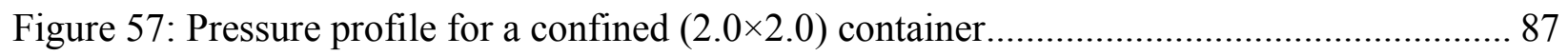

Figure 58: Pressure profile for closed, 0.25 vent area ratio and 0.5 vent area ratio ...................... 88

Figure 59: Impulse profile for closed, 0.25 vent area ratio and 0.5 vent area ratio ....................... 90

Figure 60: Single layer configurations................................................................................... 91

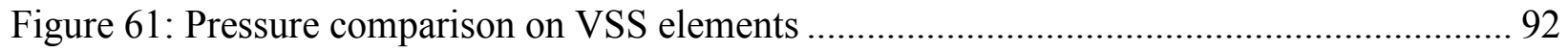

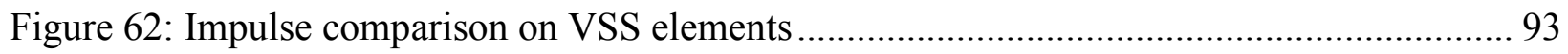

Figure 63: Comparison between the actual pressure profile from AUTODYN and the predicted pressure using the geometric coefficient for Single-layer side-by-side angle interior-facing ...... 96

Figure 64: Comparison between the actual pressure profile from AUTODYN and the predicted pressure using the geometric coefficient for Single-layer side-by-side angle exterior-facing ..... 97

Figure 65: Comparison between the actual pressure profile from AUTODYN and the predicted pressure using the geometric coefficient for Single-layer inclined plates .................................... 98

Figure 66: (a) Double-layer plate configuration, (b) Double-layer nested side-by-side angle configuration (Koger et al., 1975).......................................................................................... 99

Figure 67: Pressure profile on plates of a doubled VSS plate section ......................................... 99

Figure 68: Velocity vectors showing the jets that occurs in the double layer VSS .................... 100

Figure 69: Pressure profile on angles of Double-layer nested side-by-side angles .................... 101

Figure 70: Comparison between the available proposed equation and the actual pressure........ 103

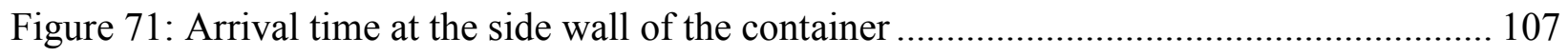


Figure 72: Pressure profile comparison between AUTODYN and proposed equations for $1 \mathrm{~kg}$ TNT in a $(3.0 \times 3.0 \mathrm{~m})$ container with vent ratio equals 0.25 109

Figure 73: Impulse profile comparison between AUTODYN and proposed equations for $1 \mathrm{~kg}$ TNT in a $(3.0 \times 3.0 \mathrm{~m})$ container with vent ratio equals 0.25 110

Figure 74: 3D model simulating a steel plate under blast load..... 113

Figure 75: Plate midpoint displacement response obtained from AUTODYN (Gauge 1)..... 114

Figure 76: Beam subjected to blast load and Equivalent Spring-Mass System. 115

Figure 77: Assumed Linear variation of acceleration during time interval, (After Paz, 1980) .. 117

Figure 78: The distinguishing limits separating thick plate, thin plate, and membrane theory, (Vantsel and Krauthammer, 2001). 120

Figure 79: A differential slice from plate (Kirchhoff plate theory) 120

Figure 80: Force-displacement relationship for an elasto-plastic single degree of freedom 122

Figure 81: Theoretical Stress Distribution for Pure Bending at Various Stages of Dynamic Loading, (UFC3-340-02, 2008) 123

Figure 82: String action in thin plates due to large deformation (ASCE, 2011)..... 124

Figure 83: Deflection of simply supported beam 126

Figure 84: Resistance-Deflection curve for steel members 127

Figure 85: Stress strain curve for steel element showing the tensile membrane action 128

Figure 86: Simplified Resistance Curve with tensile membrane 129

Figure 87: Stresses in 3D container 130

Figure 88: Comparison between obtained results from developed model and AUTODYN to predict displacement at mid span $(50 \mathrm{~mm}$ thick $)$..... 131 
Figure 89: Comparison between obtained results from developed model and AUTODYN to predict velocity at mid span $(50 \mathrm{~mm}$ thick) 132

Figure 90: Resistance-Displacement curve (50 mm thick) 133

Figure 91: Resistance-deflection curve for steel plate (10mm thick) 134

Figure 92: Resistance-deflection curve for steel plate ( $8 \mathrm{~mm}$ thick)...... 136

Figure 93: Comparison between obtained results from developed model and AUTODYN to predict displacement at mid span (8 $\mathrm{mm}$ thick)...... 137

Figure 94: Comparison between obtained results from developed model and AUTODYN to predict velocity at mid span ( $8 \mathrm{~mm}$ thick) 137

Figure 95: Resistance-deflection curve for steel plate (5 mm thick) 138

Figure 96: Comparison between obtained results from developed model and AUTODYN to predict displacement at mid span (5 $\mathrm{mm}$ thick).....

Figure 97: Comparison between obtained results from developed model and AUTODYN to predict velocity at mid span ( $5 \mathrm{~mm}$ thick) 140

Figure 98: Deflection at mid span for single layer steel plate, Single-layer side-by-side angle exterior-facing, and Single-layer side-by-side angle interior-facing (AUTODYN). 142

Figure 99: Orientation of steel angles with respect to blast loading (exterior facing configuration) 143

Figure 100: Velocity of steel angles for Single-layer side-by-side angle interior-facing VSS subjected to $10 \mathrm{~kg}$ TNT.

Figure 101: Deflection of steel angles for Single-layer side-by-side angle interior-facing VSS subjected to $10 \mathrm{~kg}$ TNT. 145

Figure 102: Velocity of steel angles for Single-layer side-by-side angle exterior-facing VSS subjected to $1 \mathrm{~kg}$ TNT. 147 
Figure 103: Deflection of steel angles for Single-layer side-by-side angle exterior-facing VSS subjected to $1 \mathrm{~kg}$ TNT.

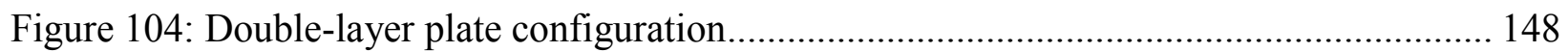

Figure 105: Deflection of steel plates in a Double-layer plate configuration............................. 149

Figure 106: Double-layer nested side-by-side angle configuration ......................................... 150

Figure 107: Deflection of steel angles in a Double-layer nested side-by-side angle configuration (AUTODYN) 150

Figure 108: Deflection of the steel plates (angles) 151

Figure 109: Tensile membrane force VS time...... 153

Figure 110: Fillet weld 156

Figure 111: Failure plane for fillet weld due to shear force 157

Figure 112: The effect of overlap on the weight. 162

Figure 113: Smoke and fire remaining after the explosion (CNN news, 2010) 174

Figure 114: Smoke from the fire, (BBC news, 2000)...... 175

Figure 115: Sierra Chemical facility burns after massive explosions (U.S. Chemical safety and hazard investigation board, 1998). 176

Figure 116: Seest fireworks explosion (Above top secrets, 2006) 177

Figure 117: Crater resulted from explosion (BBC news, 2004) ............................................. 178

Figure 118: Aerial photograph of the accident site (Mainiero et al, 2009)............................... 179

Figure 119: Highway asphalt shifts as a result of the huge explosion (Provencher, 2005) ........ 179

Figure 120: Pressure profile for $1 \mathrm{~kg}$ TNT charge in a $(2.0 \times 2.0)$ container with 0.25 vent area ratio 181 
Figure 121: Pressure profile for $1 \mathrm{~kg}$ TNT charge in a $(2.0 \times 2.0)$ container with 0.5 vent area ratio 182

Figure 122: Pressure profile for $1 \mathrm{~kg}$ TNT charge in a $(3.0 \times 3.0)$ container with 0.25 vent area ratio 183

Figure 123: Pressure profile for $1 \mathrm{~kg}$ TNT charge in a $(3.0 \times 3.0)$ container with 0.5 vent area ratio 184

Figure 124: Pressure profile for $1 \mathrm{~kg}$ TNT charge in a $(4.0 \times 4.0)$ container with 0.25 vent area ratio 185

Figure 125: Pressure profile for $1 \mathrm{~kg}$ TNT charge in a $(4.0 \times 4.0)$ container with 0.5 vent area ratio 186

Figure 126: Pressure profile for $5 \mathrm{~kg}$ TNT charge in a $(2.0 \times 2.0)$ container with 0.25 vent area ratio 187

Figure 127: Pressure profile for $5 \mathrm{~kg}$ TNT charge in a $(2.0 \times 2.0)$ container with 0.50 vent area ratio 188

Figure 128: Pressure profile for $5 \mathrm{~kg}$ TNT charge in a $(3.0 \times 3.0)$ container with 0.25 vent area ratio 189

Figure 129: Pressure profile for $5 \mathrm{~kg}$ TNT charge in a $(3.0 \times 3.0)$ container with 0.50 vent area ratio 190

Figure 130: Pressure profile for $5 \mathrm{~kg}$ TNT charge in a $(4.0 \times 4.0)$ container with 0.25 vent area ratio 191

Figure 131: Pressure profile for $5 \mathrm{~kg}$ TNT charge in a $(4.0 \times 4.0)$ container with 0.50 vent area ratio 192

Figure 132: Pressure profile for $10 \mathrm{~kg}$ TNT charge in a $(2.0 \times 2.0)$ container with 0.25 vent area ratio 193 
Figure 133: Pressure profile for $10 \mathrm{~kg}$ TNT charge in a $(2.0 \times 2.0)$ container with 0.50 vent area ratio 194

Figure 134: Pressure profile for $10 \mathrm{~kg}$ TNT charge in a $(3.0 \times 3.0)$ container with 0.25 vent area ratio 195

Figure 135: Pressure profile for $10 \mathrm{~kg}$ TNT charge in a $(3.0 \times 3.0)$ container with 0.50 vent area ratio 196

Figure 136: Pressure profile for $10 \mathrm{~kg}$ TNT charge in a $(4.0 \times 4.0)$ container with 0.25 vent area ratio 197

Figure 137: Pressure profile for $10 \mathrm{~kg}$ TNT charge in a $(4.0 \times 4.0)$ container with 0.50 vent area ratio 198

Figure 138: Pressure profile for $20 \mathrm{~kg}$ TNT charge in a $(2.0 \times 2.0)$ container with 0.25 vent area ratio 199

Figure 139: Pressure profile for $20 \mathrm{~kg}$ TNT charge in a $(2.0 \times 2.0)$ container with 0.50 vent area ratio 200

Figure 140: Pressure profile for $20 \mathrm{~kg}$ TNT charge in a $(3.0 \times 3.0)$ container with 0.25 vent area ratio 201

Figure 141: Pressure profile for $20 \mathrm{~kg}$ TNT charge in a $(3.0 \times 3.0)$ container with 0.50 vent area ratio 202

Figure 142: Pressure profile for $20 \mathrm{~kg}$ TNT charge in a $(4.0 \times 4.0)$ container with 0.25 vent area ratio 203

Figure 143: Pressure profile for $20 \mathrm{~kg}$ TNT charge in a $(4.0 \times 4.0)$ container with 0.50 vent area ratio 204 


\section{List of Tables}

Table 1: TNT Equivalent masses of some explosives (Gould et al, 1981).............................. 9

Table 2: The standard 20 and 40 feet containers, (Shipping containers 24 website)................. 19

Table 3: Properties of the developed 2D models ................................................................ 43

Table 4: Material properties of materials used in the model .............................................. 44

Table 5: Properties of the developed 3D models ................................................................. 49

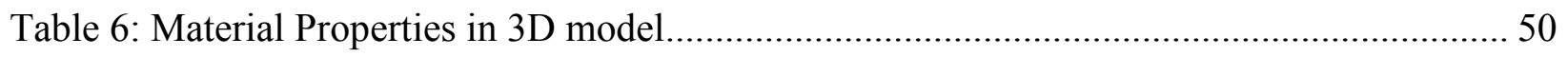

Table 7: Properties of different VSS shields with the same vent ratio 0.25 ............................ 74

Table 8: Geometric coefficient for different VSS configurations (for pressure on angles)......... 94

Table 9: Geometric coefficient for different VSS configurations (for Impulse on angles) .......... 95

Table 10: Geometric coefficient for different VSS configurations ....................................... 95

Table 11: Ratio between first and second reflected pressures ........................................... 105

Table 12: Equivalent SDOF factors for beams with fixed ends ...................................... 123

Table 13: Preliminary Design limits, UFC3-340-02 (2008) .............................................. 126

Table 14: Summary for the deflections of mid-span for steel plates for different cases ........... 158

Table 15: Summary for the deflections of mid-span for Single-layer side-by-side angle interior-

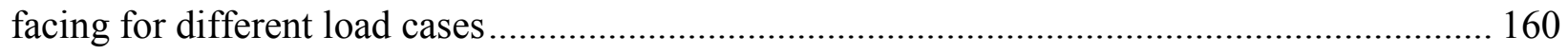

Table 16: Comparison between VSS configurations ......................................................... 162 
Abbreviations

$\begin{array}{ll}\text { AF } & \text { Aluminum Foam } \\ \text { CFD } & \text { Computational Fluid Dynamics } \\ \text { EOS } & \text { Equation Of State } \\ \text { FD } & \text { Finite Difference } \\ \text { FE, FEM } & \text { Finite Element, Finite Element Method } \\ \text { HE } & \text { High Explosives } \\ \text { IMDG } & \text { International Maritime Dangerous Goods } \\ \text { ISO } & \text { International Standards Organization } \\ \text { JWL } & \text { Jones-Wilkins-Lee (equation of state for explosive) } \\ \text { LRFD } & \text { Load and Resistance Factor Design } \\ \text { PETN } & \text { Pentaerythrite Tetranitrate } \\ \text { Q-D } & \text { Quantity Distance } \\ \text { TNO } & \text { Single Degree of Freedom } \\ & \text { Vented Suppressive Shields } \\ \text { TS } & \end{array}$




\section{Notation}

$$
\begin{aligned}
& A_{\text {vent }}=\text { Vent area in VSS section } \\
& A_{\text {wall }}=\text { Total area of a VSS section wall } \\
& a \quad=\text { Gap between VSS elements } \\
& =\text { Fillet weld throat thickness (Figure 110) } \\
& b \quad=\text { Decay coefficient } \\
& =\text { Width of the plate (Figure 78) } \\
& c \quad=\text { Damping factor } \\
& E \quad=\text { Internal energy } \\
& E \quad=\text { Modulus of elastisity } \\
& f_{y} \quad=\text { Yield stress } \\
& f_{u l t} \quad=\text { Ultimate strength } \\
& I \quad=\text { Moment of Inertia } \\
& I_{s o} \quad=\text { Incident Impulse } \\
& I_{r} \quad=\text { Reflected Impulse } \\
& l \quad=\text { Width of one element of VSS section } \\
& K \quad=\text { Stiffness } \\
& =\text { Bulk modulus of the material (Equation 17) } \\
& L \quad=\text { Total length of the VSS element } \\
& =\text { Length of weld (Figure 110) } \\
& M \quad=\text { Total width of VSS side wall }
\end{aligned}
$$




$$
\begin{aligned}
& m \quad=\text { Number of layers in a multilayer VSS section } \\
& =\text { Mass of an element } \\
& n \quad=\text { Number of elements in a single layer VSS section } \\
& P \quad=\text { Pressure (force) } \\
& P_{s o} \quad=\text { Incident Pressure } \\
& P_{r} \quad=\text { Reflected Pressure } \\
& r_{0} \quad=\text { Radius of gyration } \\
& R \quad=\text { Distance between charge and the point of interest } \\
& =\text { Radius of spherical TNT charge } \\
& R_{t} \quad=\text { Resistance in tension } \\
& R_{c} \quad=\text { Resistance in compression } \\
& r \quad=\text { Radius of gyration } \\
& T \quad=\text { Temperature } \\
& =\text { Tension force } \\
& t \quad=\text { Time } \\
& =\text { Member thickness } \\
& t_{a} \quad=\text { Arrival time } \\
& t_{d}^{+} \quad=\text { Positive duration } \\
& t_{d}{ }^{-} \quad=\text { Negative phase duration } \\
& u \quad=\text { Deflection }
\end{aligned}
$$




$$
\begin{aligned}
& \dot{u} \quad=\text { Velocity } \\
& \ddot{u} \quad=\text { Acceleration } \\
& V \quad=\text { Specific volume } \\
& W \quad=\text { TNT charge mass } \\
& X \quad=\text { Distance between charge and the side wall of a box container } \\
& Z \quad=\text { Scaled distance } \\
& \rho \quad=\text { Density } \\
& \rho_{0} \quad=\text { Reference density } \\
& \alpha_{i} \quad=\text { Vent area ratio of single layer VSS section } \\
& \alpha_{e f f}=\text { Vent area ratio of multilayer VSS section } \\
& \omega \quad=\text { Adiabatic constant } \\
& \Delta \quad=\text { Delta (change) } \\
& \Delta_{e} \quad=\text { Elastic deflection } \\
& \Delta_{\max } \quad=\text { Maximum deflection } \\
& \Delta_{t} \quad=\text { Time increment } \\
& \gamma \quad=\text { Ratio of specific heats } \\
& \sigma_{x}, \sigma_{y}=\text { Principal Stress in } \mathrm{X} \text { and } \mathrm{Y} \text { direction, respectively } \\
& \tau_{x y} \quad=\text { Shear stress } \\
& \varepsilon \quad=\text { Strain } \\
& \zeta \quad=\text { Damping ratio } \\
& v \quad=\text { Poisson's ratio }
\end{aligned}
$$




\section{Chapter 1: Introduction}

\subsection{Background}

Vented Suppressive Shields (VSS) provide one of the best solutions for the storage and transportation of hazardous materials. The main role of the VSS containers is to attenuate the blast pressure and impulse outside of the container resulting from the accidental explosion of hazardous materials as well as containing fragments resulting from the explosion within the VSS container. However, there are no comprehensive procedures or guidelines for design of VSS containers to attenuate the blast loading on proximate buildings and population, contain primary fragments from the explosion, and resist the imposed blast loading on the VSS container elements in a cost-effective manner. The existing VSS containers are designed from experience and observations of previous container test programs or lessons learned from previous accidents. Design of VSS containers has been a challenging task for structural engineers due to the lack of the understanding the interaction between the blast waves and the elements of the VSS container and thus difficulty in determining the load on the elements of the VSS container.

As the name suggests, "Vented Suppressive Shield", are containers with vents or openings in their sections. The effectiveness of vented suppressive shield (VSS) sections in attenuating blast pressure outside a container depends mainly on the vent area ratio or effective vent area ratio in multi-layer suppressive shields. Calculating the vent area ratio has been challenging for many VSS sections due to the complexity of the geometric shape of the section. Guidance is available for only few suppressive shield configurations used by earlier researchers. Thus a method is required for calculating the effective vent area ratio of suppressive shields, allowing the study of more diverse configurations.

Depending on the amount of openings within the VSS section, the level of attenuation of blast loads is determined for the given VSS section. However, different VSS sections with the same effective vent area ratio may achieve different levels of attenuation of blast loads. Moreover, the effective vent area ratio and the geometric configuration affects the reflected pressure profile on the side wall of a cubicle VSS container and thus affects the structural response of those elements. This is due to the difference in the angle of reflection as well as the exposed area of the element that is subjected to the blast load. AUTODYN has been used to 
study the interaction between blast loads and structural elements; however, there are two main disadvantages in using AUTODYN software. The first is that those packages are very expensive and they require knowledge and specific training to be efficiently used. The other is that AUTODYN doesn't have the capability to provide the reactions and forces on the structure that are important for the design stage of the walls, roof, main frame and welded connections in the VSS container.

\subsection{Research Goals and Approach}

The main objective of this research is to develop a simple design method to design steel vented suppressive shields that can be used by individuals without the need of using expensive/complex software packages. The design method is able to obtain a structural response of VSS elements that is comparable to those obtained from AUTODYN. Moreover, the design method will extend to calculate the reactions at the end supports of the VSS elements that will be used in the design of the welding part. The objective of the thesis is achieved through a series of targeted research investigations listed below:

1) Calculating the effective vent area ratio of the studied VSS,

2) Investigating the performance of different VSS sections,

3) Predicting the loading on the VSS elements due to internal explosion,

4) Introducing a SDOF model to study the behavior of the VSS elements due to blast loads,

5) Design the welding of the VSS sides to the main frame of the container.

The study is mainly a numerical and analytical investigation. The numerical part includes the use of AUTODYN software. The first part of the study is a two dimensional (2D) numerical modelling to study the pressure outside the container and on the side walls of the VSS container. Different cases for VSS containers were modeled and the pressure profiles were recorded at different locations outside and inside the container. From the obtained pressure profiles, a set of equations was introduced that can be used instead of the CFD code to compute the pressure and Impulse outside the container as well the pressure profile on the side walls of VSS containers. Another set of equations was proposed to calculate the pressure profile on the side wall of VSS containers. 
Following obtaining the pressure profiles, three dimensional (3D) AUTODYN models were developed to obtain the deflections and velocities of deformations of VSS elements due to blast loads. The 3D modeling part shows the deflections of the VSS elements as well as the stresses on these elements.

The analytical part of this study is developing a SDOF model to predict the structural response of the side walls elements of the VSS container. The SDOF model was able to predict the deflections and velocities of the VSS elements forming the side wall of the VSS container and the results are validated by comparing it with those obtained from AUTODYN software.

The welding design depends on the reactions obtained at the end supports of the side wall of VSS container. These end reactions will be obtained from the developed SDOF. The welding between the VSS elements and the main frame of the container was designed using the Handbook of Steel Construction (CAN/CSA S16-01, 2004). Figure 1 shows the flow chart for the research plan of the thesis.

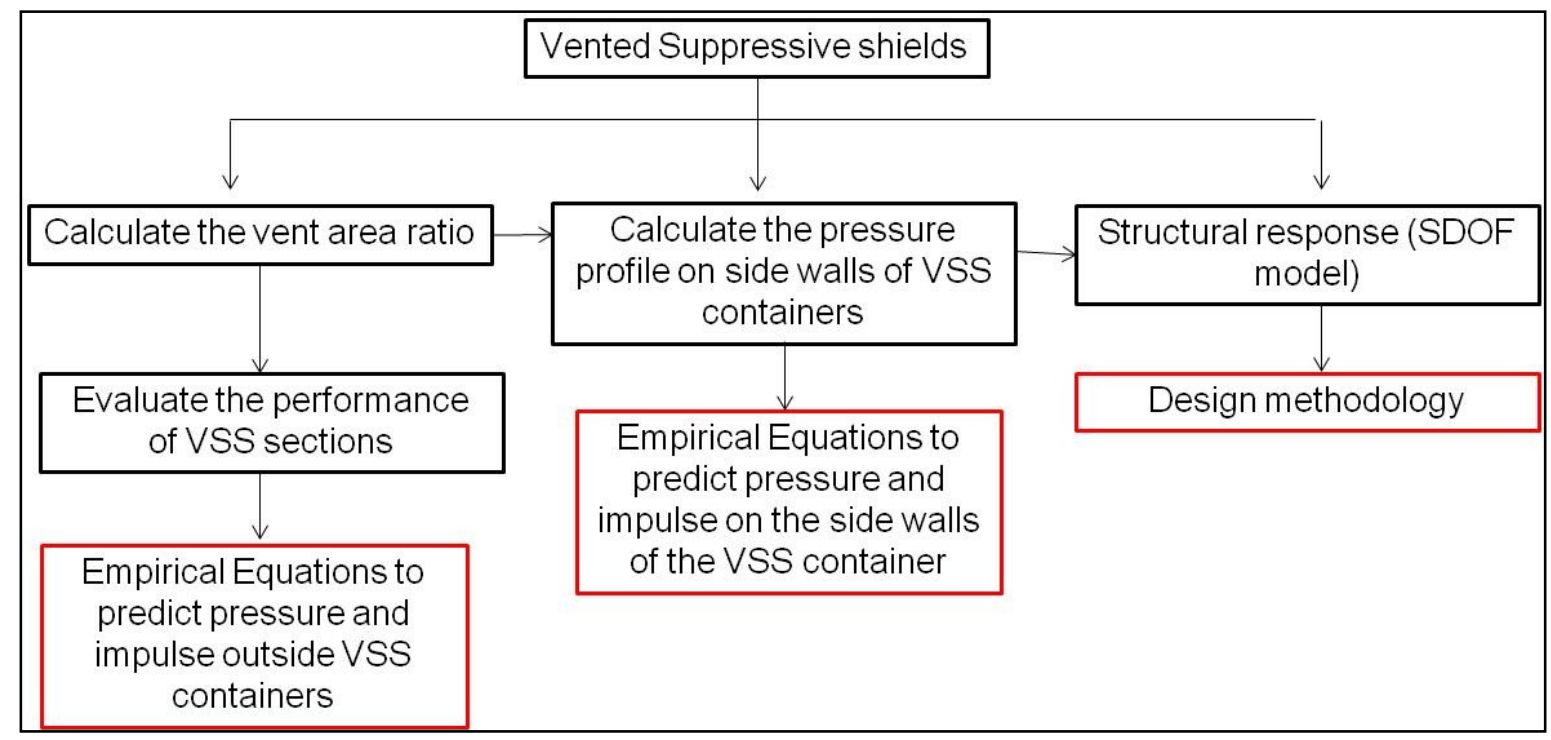

Figure 1: Flow chart for the research plan of the thesis

\subsection{Thesis organization}

The thesis consists of seven chapters. Chapter 2 presents a comprehensive literature review of past industrial and transport accidents involving the explosion of hazardous materials; especially explosives and other energetic materials such as fireworks. Chapter 2 also reviews the 
previous research work involving internal explosion in confined structures. Both experimental research works and numerical analyses conducted to study the effects of internal explosion on the containers and proximate structures and populations are reviewed. Also, the literature review investigates the development of vented suppressive shields for attenuation of blast pressure and impulse outside of the containers.

Chapter 3 includes a detailed presentation and discussion of the 2D and 3D AUTODYN models that were developed in the study. This includes an explanation to the techniques used to study the detonation process, blast wave propagation in air, and blast wave interaction with the side walls of the VSS sections.

Chapters 4 and 5 present the results obtained from AUTODYN models as well as detailed discussion of these results. Using the results obtained from AUTODYN, sets of empirical equations are presented in the two chapters to predict the pressure and impulse outside the VSS containers and the pressure on different VSS sections.

In Chapter 6, a single-degree-of-freedom (SDOF) model to study the structural response of the steel elements of the VSS shield is presented. The results obtained from the SDOF model are compared to those obtained from AUTODYN.

Finally, the conclusions and the recommended future work are summarized and discussed in Chapter 7. 


\section{Chapter 2: Literature Review}

\subsection{Introduction}

Storage and transportation of hazardous materials is becoming one of the most important issues that affects the security and economy of many countries around the world. Hazardous materials, especially explosives, have a capacity to detonate in storage or during transport. Currently many containers used in the transport of explosives and hazardous materials are not specifically designed to withstand internal explosions. Using regular containers in the transportation and storage of hazardous materials leads to huge losses in case of explosion accidents (Mainiero, 2009). Depending on the quantity of explosive materials, the containments can be completely broken up and thrown from the centre of detonation with potential to damage surrounding infrastructures and cause injury and fatality to people. To mitigate the effects of accidental explosions, it is essential that containers used for the storage and the transportation of dangerous goods be specially designed to be capable of mitigating the hazards associated with blast loading. The more robust a container is the better its performance in terms of safety and security of the transported materials, surrounding infrastructures and proximate population. A container designed for explosives storage or transport should have the capacity to attenuate and mitigate the explosion effects outside the container while remaining intact and not contributing to the debris field. In order to achieve an appropriate design for hazardous material containment structures, a good understanding of explosions, their properties and effects on structures and humans is required. The current state of understanding of explosion properties and their effects on infrastructure systems is discussed in details in the following section.

\subsection{Classification of Explosions}

An explosion is defined as a rapid increase in volume due to a sudden release of energy in an extreme manner that is usually accompanied with the generation of high temperatures, high pressures and release of gases in a short period of time (Baker, 1983). An explosion can be categorized as physical, nuclear or chemical depending on its cause. For physical explosions, energy is released from either failure of a compressed gas cylinder, volcanic eruptions or mixing of two liquids at different temperatures. In the case of nuclear explosions, energy is released due to the splitting or formation of different atomic nuclei by the redistribution of the protons and 
neutrons within the interacting nuclei, whereas the rapid oxidation of fuel elements (carbon and hydrogen atoms) are the main source of energy release in case of chemical explosions (Baker, 1983).

Another categorization for explosive materials is according to their physical state; where explosives are categorized as solids, liquids or gases. Solid explosives are mainly high explosives for which blast effects are best known. Explosives are also classified on the basis of their sensitivity to ignition as primary or secondary explosives. Primary explosives are those that can be easily detonated with least energy from a spark, flame or impact. Mercury Fulminate and Lead Azide are examples of primary explosives. Secondary explosives are less sensitive in comparison with primary explosives and require substantially more energy (impetus) to be initiated. Because of this, secondary explosives are usable in a wide variety of applications and are safer to handle and store, however they are usually initiated by a small quantity of a primary explosive. An example of secondary explosives is Trinitrotoluene (TNT). Mostly, people pay more attention to protect the more sensitive primary explosives; however the secondary explosives can be more dangerous and can result in widespread damage to surrounding infrastructures because of their quantities in storage and transport (Baker, 1983).

\subsection{Detonation of explosives}

When a high explosive is detonated, it generates hot gases under high pressure, up to $30,000 \mathrm{MPa}$, and temperature of approximately $3000-4000^{\circ} \mathrm{C}$. The hot gases expand very rapidly forcing out air in the volume it occupies. As a result, a thin layer of compressed air is formed in front of the expanding volume of gas and contains most of the energy released by the explosion. The compressed layer of air is known as the shock front (Kinney and Graham, 1985). When the shock front reaches a point in air, the pressure instantaneously increases to a value above the ambient atmospheric pressure. This increase in pressure is referred to as "side-on overpressure" (Kinney and Graham, 1985) and it decays as the shock wave sweeps from one point to another and expands outward from the explosion source. After a short time, the pressure behind the shock front may drop below the ambient pressure forming a region of negative pressure. The period during which pressure is below atmospheric is characterised by the creation of a partial vacuum and air suction. 
Figure 2, shows a typical blast pressure profile recorded at a station remote from the centre of explosion. The blast pressure profile (waveform) can be completely characterized by three parameters; overpressure, duration and impulse. The time, $t_{a}$, represents the time of arrival of the shock front at the recording station. The overpressure is denoted by $P_{s o}$, while the durations of the positive and negative phases are denoted by $t_{d}{ }^{+}$and $t_{d}$, respectively. The negative phase duration is typically longer than the positive phase duration; however the negative phase overpressure is substantially lower than the positive phase overpressure. The negative phase is less important for most structures, but should be considered for light-weight structures where it could cause failure of the structure. The impulse of the blast wave is calculated as the area under the blast pressure - time profile.

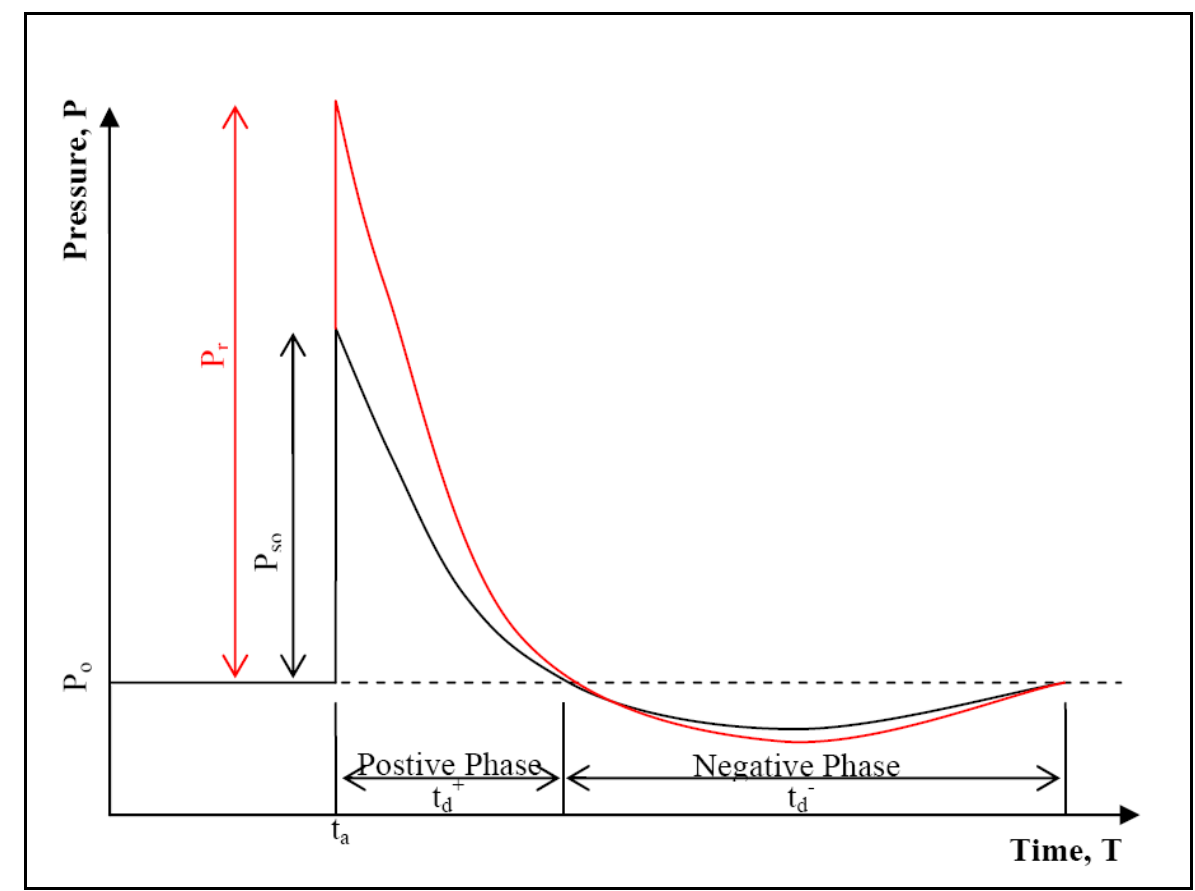

Figure 2: Typical blast wave profile (Braimah, 2012)

\subsection{Reflected Blast Waves}

Blast waves, as other kind of waves, reflect when they encounter an object denser than the medium in which they are traveling. Moreover, blast waves diffract around objects of finite dimensions. Reflected pressure is known to be much higher than the incident pressure as shown in Figure 2. This is because when the blast wave hits a target, the air molecules in front of the 
encountered object are compressed and thus the pressure increases. The reflection and the diffraction of the blast wave depend mainly on the size and the orientation of the encountered object relative to the direction of travel of the wave. The reflected pressure coefficient, $C_{r}$, is used to calculate the magnitude of the reflected pressure in cases where the incident blast wave does not impinge normally on the reflecting surface. The reflected pressure coefficient depends on the orientation of the surface with respect to the direction of the blast wave and the magnitude of incident blast pressure (Kinney and Graham, 1985). The reflected pressure is used in the design of most structural elements.

\subsection{TNT Equivalency}

Generally, the magnitude of an explosion is expressed by the amount of energy released in the detonation of the explosive. There are different kinds of explosives in use today and each one of them has its own energy density. In order to simplify this difference, the magnitude of any explosive substance is expressed relative to a standard explosive (Sochet, 2010). The generally accepted standard explosive is Trinitrotoluene that is known by TNT. TNT is a relatively pure, safe to handle, and available explosive. Also, a lot of testing has been carried out using TNT and thus a lot of data is available for blast wave parameters obtained from TNT detonations. TNT has been used as standard explosive for decades.

Extensive research has been carried out to determine the energy produced from the detonation of TNT. Researchers have reported blast energy from TNT detonation to vary from 4610 to 4680 Joules per gram (Maruyama, 2000). The difference between these limits is attributed to differences in the atmospheric conditions while carrying out the experimental tests. The magnitude (energy density) of other explosives is expressed relative to that of TNT and is known as "TNT equivalence". TNT equivalencies are used to convert the amount of an explosive type into an equivalent amount of TNT required to produce an equivalent effect. TNT equivalence can be dependent on the heat of combustion, heat of detonation (relevant to peak pressure and impulse), and detonation energy (Maienschein 2002). TNT equivalence for overpressure and impulse depends mainly on the stand-off distance to the measuring station (Wharton et al., 2000). Table 1 shows the TNT equivalency of different types of explosives for both pressure and impulse. 
Table 1: TNT Equivalent masses of some explosives (Gould et al, 1981)

\begin{tabular}{|c|c|c|c|}
\hline Explosive & $\begin{array}{c}\text { Density } \\
(\mathrm{g} / \mathrm{cm} 3)\end{array}$ & $\begin{array}{c}\text { TNT Equivalent } \\
\text { for pressure }\end{array}$ & $\begin{array}{c}\text { TNT Equivalent } \\
\text { for Impulse }\end{array}$ \\
\hline ANFO & 1.77 & 0.82 & 0.82 \\
\hline Amatol 60/40 (60\% ammonium nitrate, 40\% \\
TNT) & 2.5 & 0.586 & 0.586 \\
\hline Comp B (60\% RDX, 40\%TNT) & & 1.11 & 0.98 \\
\hline C4 (91\% RDX, 9\% Plasticizer) & 1.05 & 1.37 & 1.19 \\
\hline $\begin{array}{c}\text { H-6 (45\% RDX, 30\% TNT, 20\% Al, 5\% D-2 } \\
\text { wax) }\end{array}$ & 1.76 & 1.38 & 1.15 \\
\hline HBX-1 (40\% RDX, 38\% TNT, 17\% Al, 5\% D-2 \\
wax) & 1.75 & 1.17 & 1.16 \\
\hline Octol, 75/25 (75\% HMX, 25\% TNT) & 0.824 & 1.06 & 1.24 \\
\hline PETN & 1.77 & 1.24 & 1.00 \\
\hline Pentolite, 50/50 (50\% PETN, 50\% TNT) & 1.77 & 1.42 & 1.09 \\
\hline RDX (Cyclonite) & 1.76 & 1.14 & 0.96 \\
\hline TNT & 1.65 & 1.00 & 1.07 \\
\hline Tritonal (80\% TNT, 20\% Al) & 1.80 & & \\
\hline
\end{tabular}

\subsection{Internal explosion}

Internal explosion refers to the case where an explosion occurs within a confined structure. Internal explosion is considered to be a special case of blast loading and it is also known as confined explosion. The difference between internal explosion (confined explosion) and free air-burst is that in free air-burst the blast wave strikes the target causing a high reflected pressure followed by exponential pressure decay. In this case, the target is only affected by one reflected shock wave that lasts for a very short duration but with a high magnitude (Kingery et al., 1975).

In case of internal explosion (Figure 3), the pressure waves hit the sides of the enclosed structure and reflect back towards the center of detonation where the reflected waves collide with each other and reflect again towards the side wall of the structure. The reflected waves keep reflecting inside the closed structure until the pressure dissipates and thus the walls of a confined structure are exposed to more than one reflected wave as in case of free air-burst explosion. So it can be said that in case of internal explosion, the target (side walls and roof of the confined structure) is exposed to blast loads for a longer duration compared to that of free air-burst (UFC3-340-02, 2008). Figure 3 shows the reflection of blast waves on the interior sides of a containment structure. 


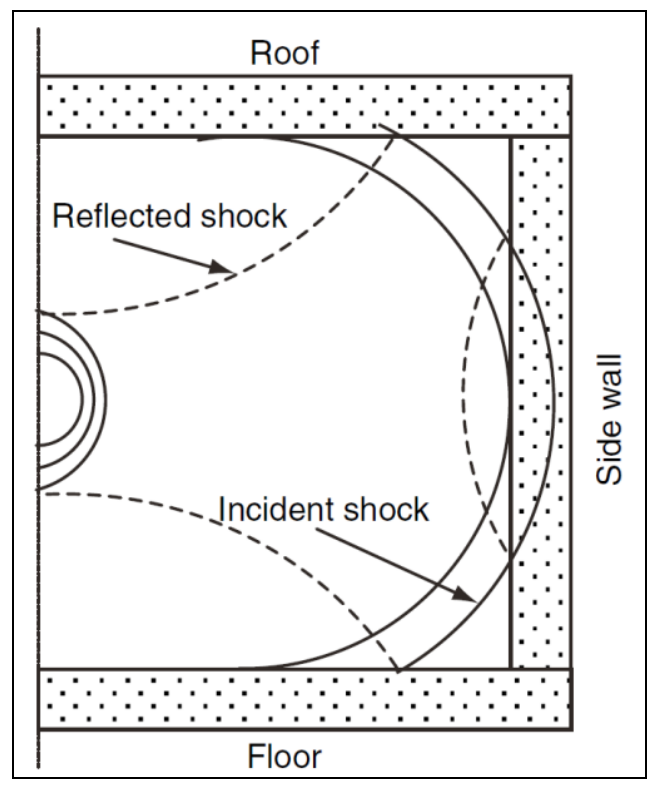

Figure 3: Blast wave reflection within confined structure (Braimah et al, 2012)

The loading resulting from internal explosion consists of two phases; the first phase is the reflected pressure (shock) phase that consists of several pulses of very complex waveform due to the complication of the reflection process of the shock wave within the structure. The second phase is the quasi-static (gas) phase where the gas products build up forming additional pressure inside the confined structure. Figure 4 shows the pressure profile for an internal chemical explosion at the center of a closed container. This figure shows three distinct pressure regions (Kinney and Graham, 1985). The first region is indicated by (A) showing the initial pressure rise that occurs due to the chemical combustion reaction produced from the explosion. The high pressure region, marked by (B), occurs when the reflected waves reflects back from the side walls back to the center of detonation (center of the container). The third region defined by part (C), is that of pressure decay that results from the pressure relief effects as gases escape through leaks in the containment due to some deformations in its structural walls or due to cooling of the gas products. Comparing the pressure - time curve for both typical (free air-burst) blast wave (Figure 2) and internal explosion (Figure 4), it is observed that the pressure profile in case of free air explosion has only one peak while for the internal explosion pressure profile, it has more than one peak. This makes the pressure dissipates in shorter time for the free air blast load compared to the case of an internal explosion. This results in higher impulse values in case of internal explosion (Kingery et al., 1975). 


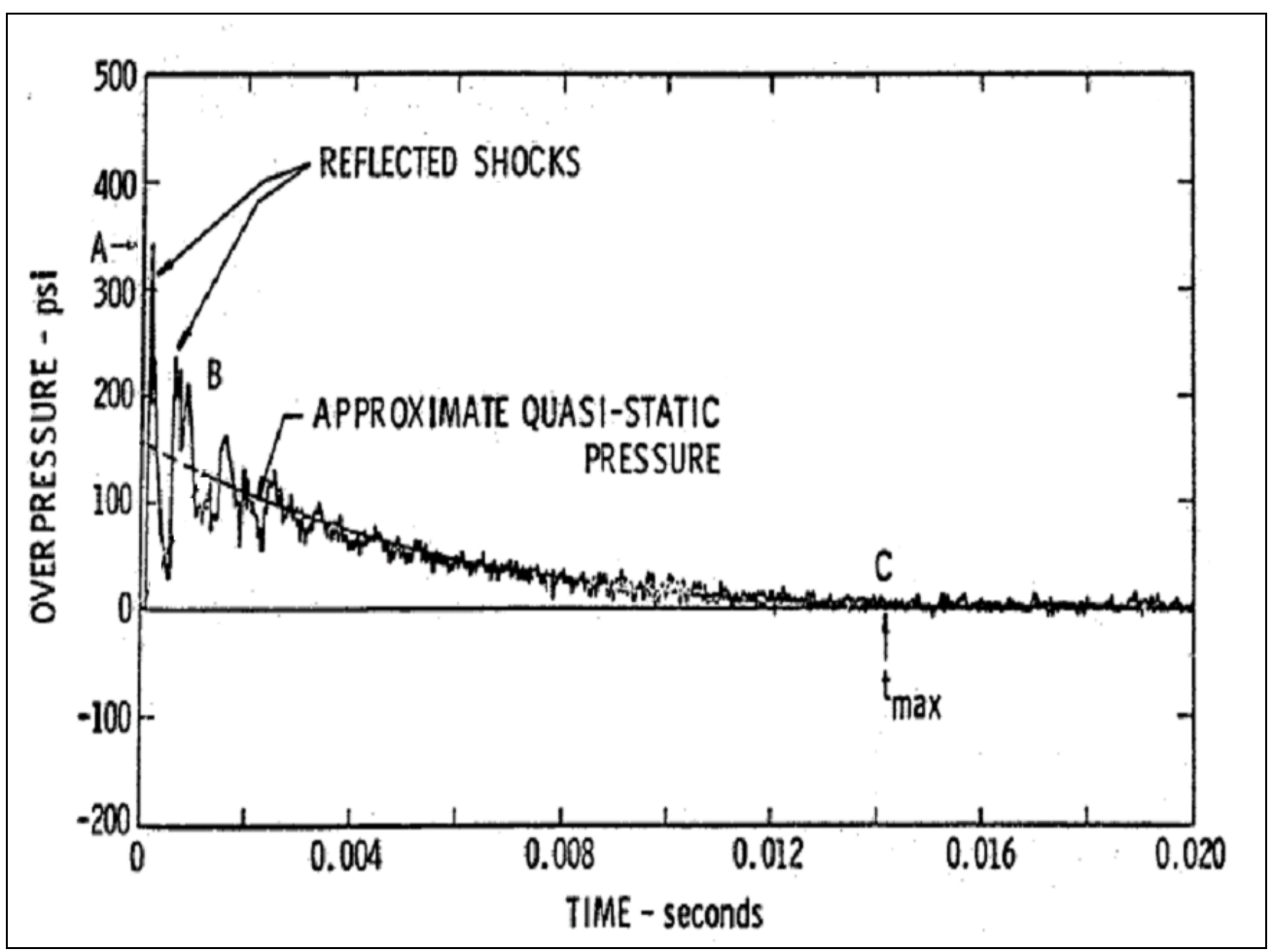

Figure 4: Typical time history for internal pressure at inner surface of enclosed structures, (Kingery et al., 1975)

Another important component to be taken into consideration when dealing with internal explosion is the gas pressure. In case of free air-burst explosion, the gas pressure dissipates rapidly. However, in case of internal explosions, the gas products build up and the temperature within the structure rises. This leads to the formation of blast pressures whose magnitude is generally less than that of the shock pressure but whose duration is significantly longer. As long as the explosion is completely confined, there is no way for the internal gas pressure to escape outside the structure. This buildup of the gas pressure may lead to the failure of the walls of the structure even if it is able to resist the shock wave from the explosion (UFC3-340-02, 2008).

According to UFC3-340-02, 2008, the actual duration and the pressure-time variation of the gas pressures are not required for the analysis of most structural elements. Similar to the shock pressures, the actual pressure-time relationship can be approximated by an equivalent triangular pulse. Figure 5 illustrates an idealized pressure-time curve considering both the shock and gas pressures (UFC3-340-02, 2008). 


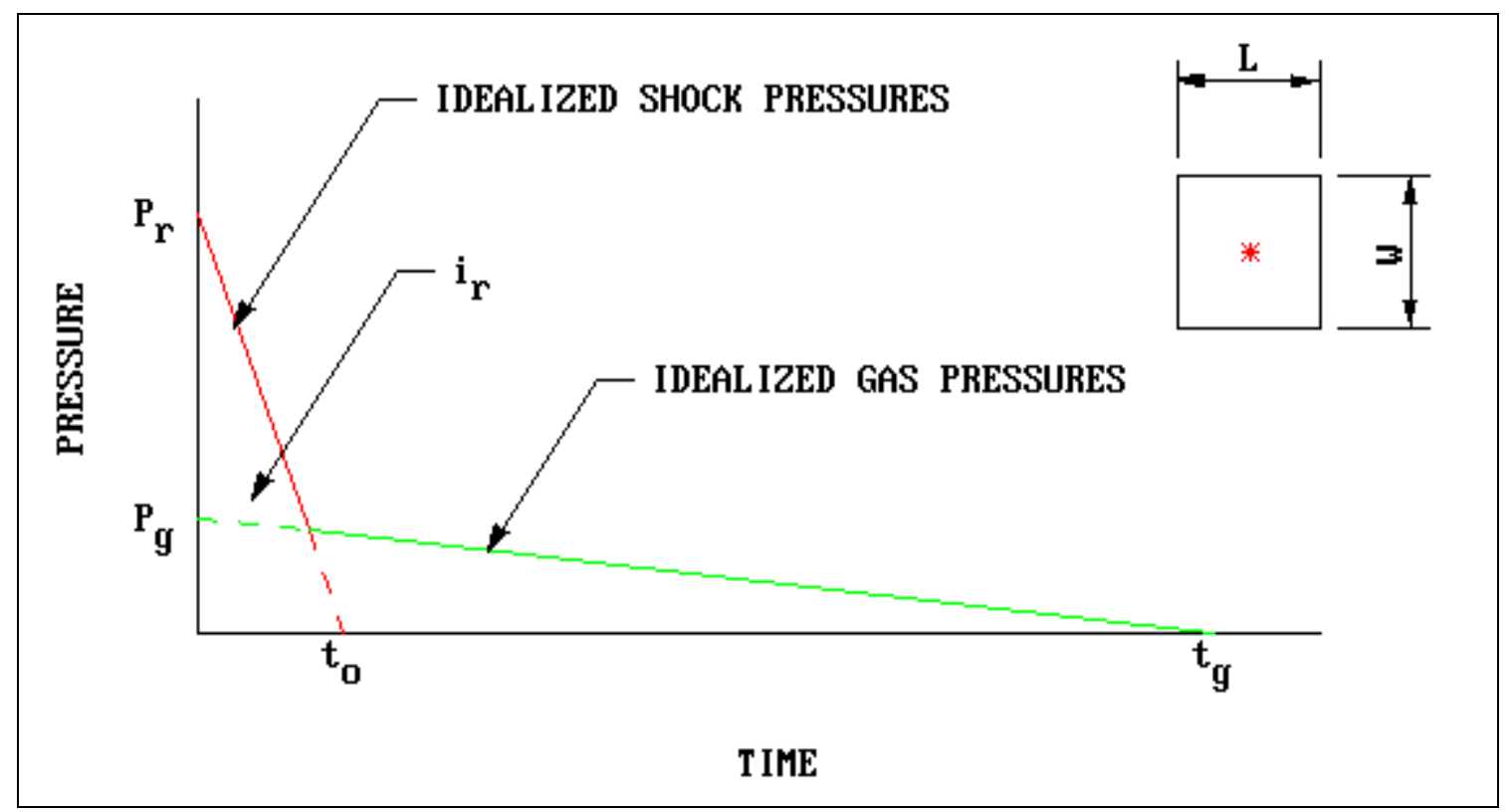

Figure 5: Combined shock and gas Pressures (square chamber), (UFC3-340-02, 2008)

If a chamber is square in plan (cube) as shown in Figure 5, then the magnitude of the gas pressure acting on an individual element will not vary significantly along the length of the elements. For design purposes, the gas pressures may be considered to be uniform on all walls and roof elements. When the chamber is longer in one direction (rectangular cuboid) and the explosion occurs at one end of the structure, the magnitude of the gas pressures will vary along the length of the structure. At the end, where the explosion occurs, the peak gas pressure is $P_{g 1}$ (Figure 6) which after a finite time decays to $P_{g 2}$, and finally decays to zero. The length of time $t_{p}$ between the two peak gas pressures may be taken as the length minus the width of the structure divided by the velocity of sound. 


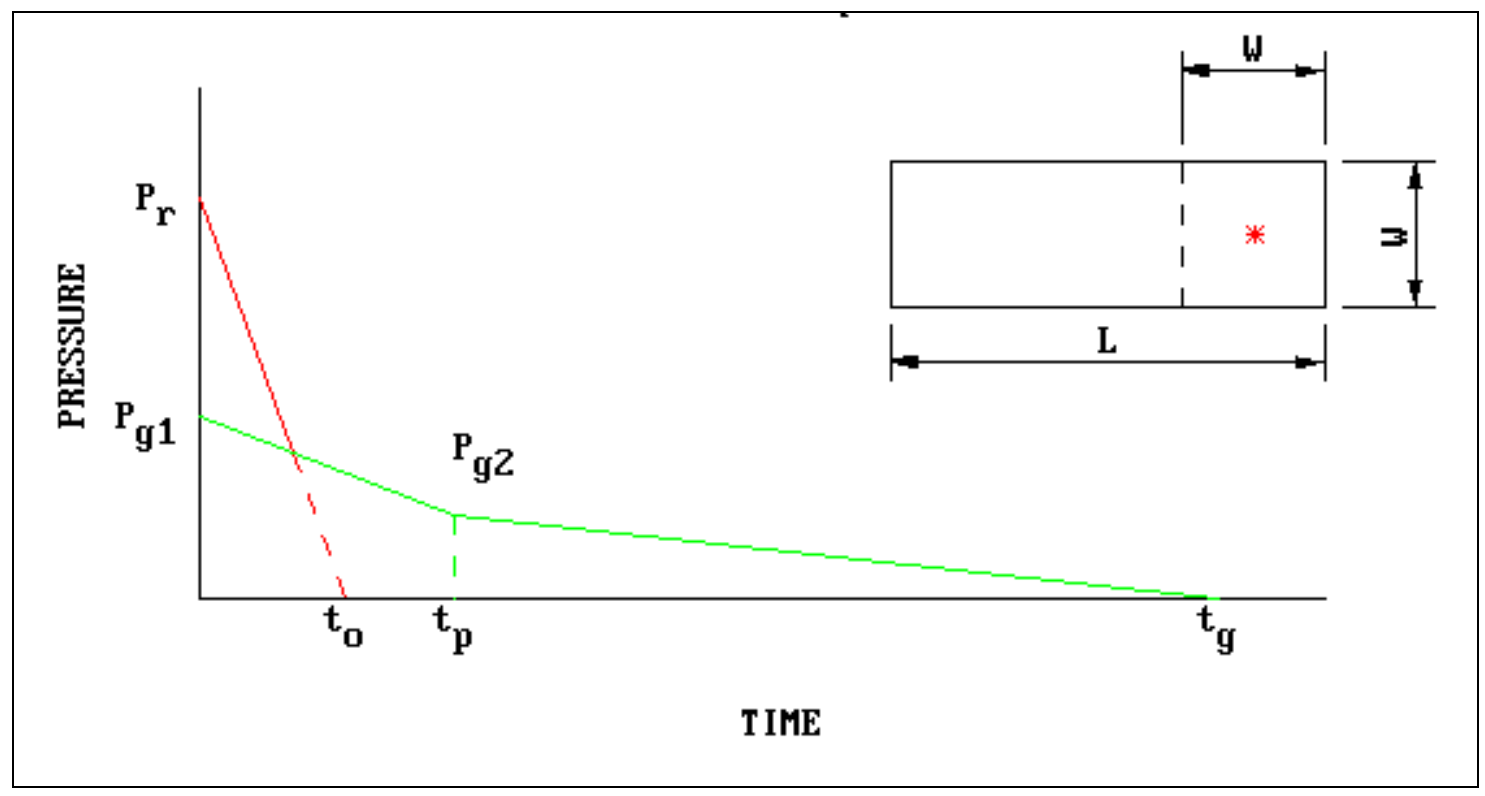

Figure 6: Combined shock and gas pressures (long rectangular chamber), (UFC3-340-02, 2008)

One of the practical solutions to overcome the problem of the accumulation or the build up of gas pressure is to allow some of the gas pressure to vent outside the structure. This can be done by having openings within the side walls of the structure. These openings enable venting of gas pressure to the outside and thus no gas pressure accumulation takes place.

\subsection{Explosion Accidents}

Accidents in the storage or the transportation process may lead to accidental explosion and can cause damage of surrounding infrastructure as well as injury and death to people in the vicinity of the explosion. These accidents are usually unpredictable, but from studying previous accidents, the risks of accidental explosions and their hazards and effects on proximate infrastructure systems and population can be estimated. Regulations have been developed to reduce the fatalities and loss that could happen if an accident occurred. One of these regulations is the "International Maritime Dangerous Goods" (IMDG) Code that was developed as a uniform international code for the transport of dangerous goods by sea (International Maritime Organization, 1998). This code covers such matters as packaging, container traffic and storage, with particular reference to the segregation of incompatible substances. 


\subsubsection{Accidents during Storage}

The storing process of materials is generally designed to keep the surrounding safe and to keep the materials inside away from effects that could damage them, i.e. humidity, heat and rain.. During storage there is little risk for mistakes causing damage or initiation of hazardous materials in the storage. However, these containers could be exposed to heat or impact due to surrounding effects.

The latest recorded accident related to the storage of hazardous materials or dangerous goods was reported in July 2011. The accident occurred in the Evangelos Florakis Naval Base in Zygi and is considered to be the worst peacetime military accidents ever recorded in Cyprus. The accident occurred when 98 containers of explosives, stored for $2 \frac{1}{2} 2$ years in the sun, exploded due increased temperature inside the storage rooms. The explosives were stored in regular containers that were not designed to contain an internal explosion. The accident resulted in the death of at least 13 people, injury of another 62 and the damage of a local Electrical power station (Demotix, 2011)

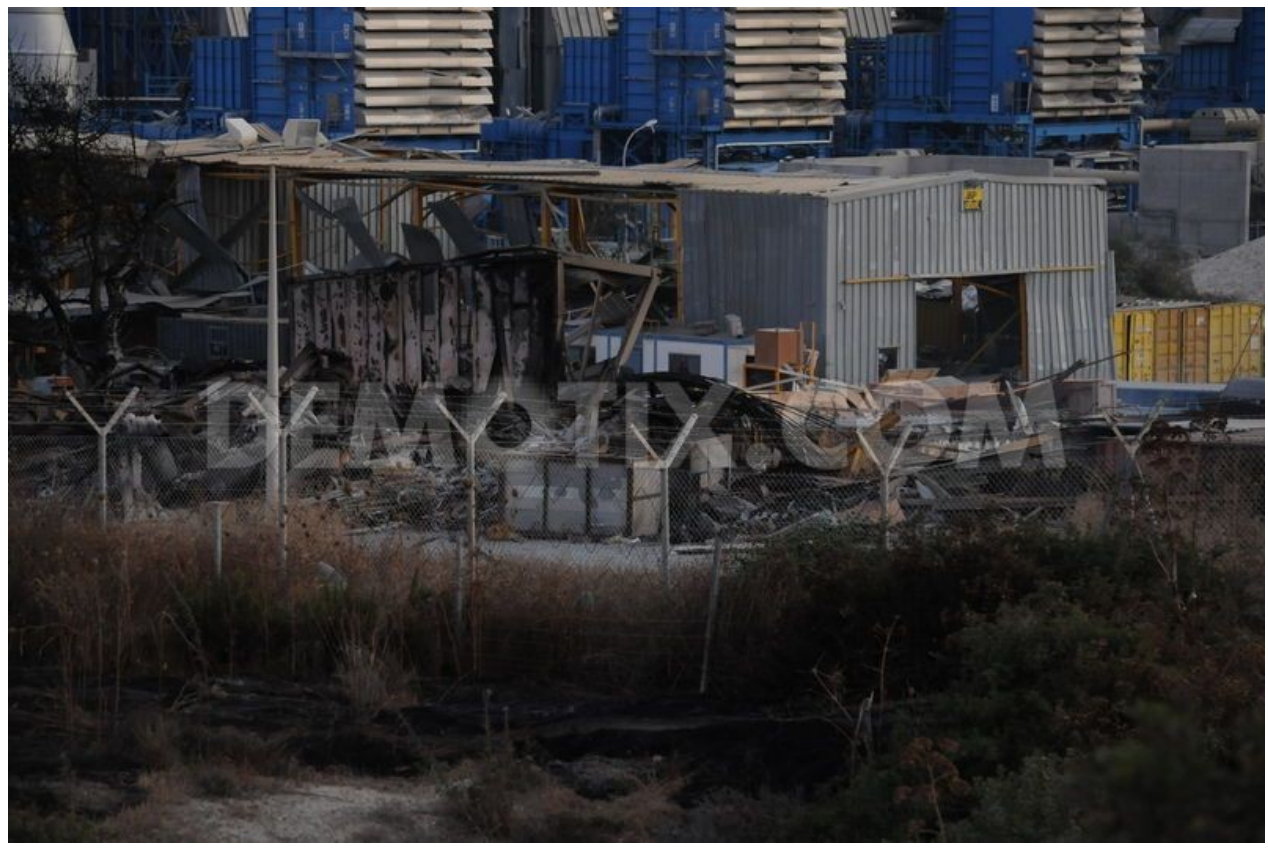

Figure 7: Explosion at the Evangelos Florakis Naval Base in Cyprus (Demotix, 2011) 


\subsubsection{Accidents related to the transportation}

Explosion accidents during transportation are more common than those in storage. This is due to the risks and hazards associated with transportation of explosives (hazardous materials). Even though many countries, including Canada, have very strict regulations guiding the transport of hazardous materials, accidents involving dangerous goods during transportation is not uncommon. The following section presents accidents of dangerous goods in transport to highlight the inherent hazards and vulnerabilities of the containment systems.

\subsubsection{Fireworks truck explosion in China}

In February 2013, a truck transporting fireworks was involved in an accident on the Yichang Bridge in eastern China. The accident caused a fire in the truck leading to explosion of the fireworks. . The explosion resulted in failure of the Yichange Bridge - a reinforced concrete pre-stressed Girder Bridge. An 80-m section of the bridge, $28 \mathrm{~m}$ above ground level collapsed. Nine people were killed in this accident while another 26 were severely injured (The New York Times, May 2013).

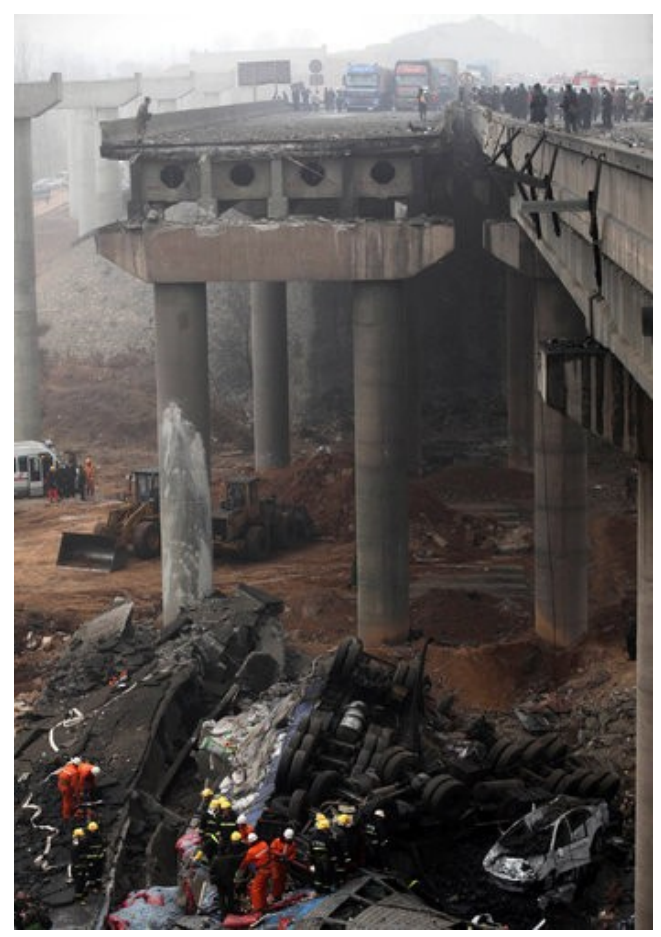

Figure 8: The damage in the bridge due to the explosion (New York Times, 2013) 


\subsubsection{M/V Hyundai Fortune}

The M.V. Hyundai Fortune was a 64,054 tones Panama flag container vessel that was built in 1996. On March 2006, the container vessel exploded near the Gulf of Aden on a west bound voyage from Singapore to Amsterdam and other European ports. The vessel was carrying approximately 3,250 loaded intermodal containers, including 7 container loads of fireworks from China. The reason for the explosion was the transfer of heat to the cargo. The accident investigation concluded that the heat was due to fire in the engine room that transferred to the cargo and caused the explosion. The explosion caused many containers to be blown into the sea. The loss in cargo due to the fire and explosion was estimated to be about $\$ 100$ million plus the value of the vessel itself which was estimated to be around $\$ 50$ million. Although there were no human casualties, this accident caused severe economic losses. After the accident a global suspension on the movement of all fireworks shipments was enforced (Weeth \& Associates LLC, 2006). This decision negatively affected the fireworks industry and summer festivals around the world. The summer festivals are of great importance to the tourism industry and contribute heavily to the national economy of many developed countries.

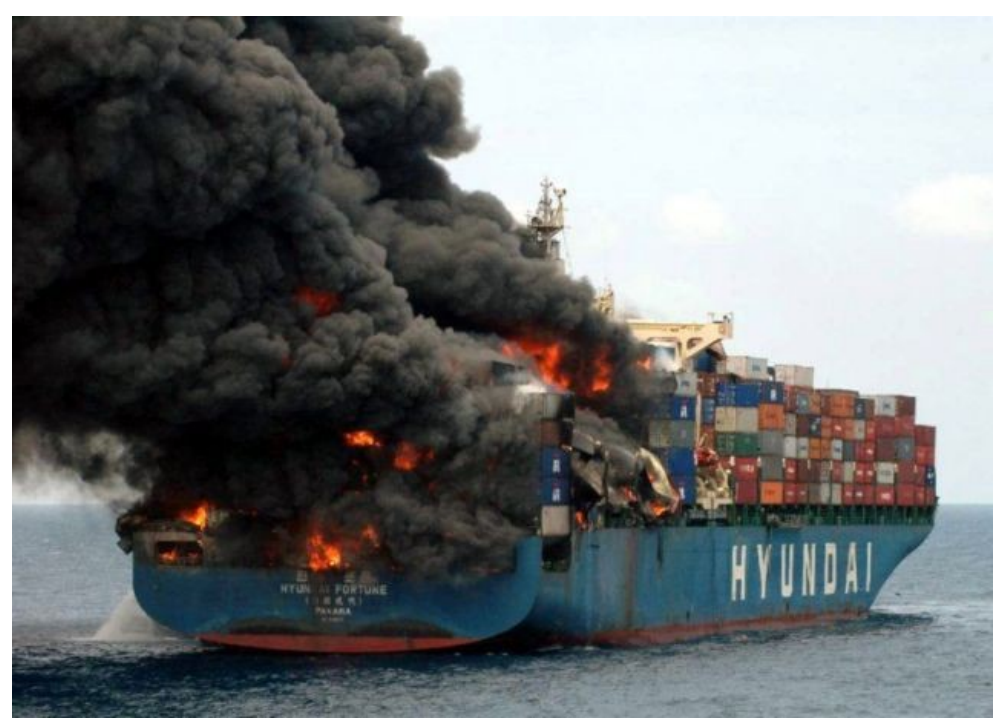

Figure 9: Damage of the body of vessel (Weeth \& Associates LLC, 2006)

More accidents that are related to the transportation and storage of hazardous materials are mentioned in Appendix A. 


\subsubsection{Summary of accidents}

The mentioned accidents show the sequence of events that could lead to an explosion. There is a potential to significantly reduce injuries and fatalities if the time between the accident (fire) and explosion was used for evacuation rather than fighting the fire as in case of the truck explosion in Walden, Ontario. It is evident that the main causes of accidents occurring during transportation or storage of hazardous materials can be attributed to one of the following reasons:

- The high temperature due to hot weather or fire or the presence of another source of heat energy beside the containment (Storage or Transportation).

- Collision accidents (Transportation).

- Slips and trips during handling of hazardous materials (Transportation).

\subsection{Quantity Distance (Q-D)}

To mitigate the risk of damage to surrounding infrastructure, quantity-distance (Q-D) relations are used to identify safe areas around explosive processes for the population and transport routes. Quantity-distance expresses the relationship between the quantity of explosives stored and the distance from the centre of detonation at which the blast pressure and fragment hazards are below a prescribed level. This relationship is based on levels of risk considered acceptable for the stipulated exposures (US Department of Defense, 2008). The area is usually circular with a specific radius from the centre of detonation. The Quantity-Distance is dependent on the quantity and type of explosives stored and the level of blast pressure deemed acceptable. The higher the quantity of explosives stored the greater the distance requirements. Because the cost of land is becoming expensive in populated areas, the main goal is to reduce the quantity distance requirements of the explosives industries. One of the solutions to reduce this distance is to store those hazardous materials in structures that are able to contain the blast load and fragments in case of an accidental explosion. There are two types of quantity distances inside and outside quantity distances (Kasemets, Natural Resources Canada, 2008). 


\subsubsection{Inside Quantity Distances}

Inside quantity distances are the distances separating the donor and other surrounding explosives in the same area. Inside quantity distances are divided into two types (Kasemets, Natural Resources Canada, 2008):

- Inter-Magazine Distances: They are applied between a donor and other explosives storage sites such as magazines, container or confined structures.

- Process Building distances: They are applied between a donor site and a facility where explosives are being manufactured or worked on or between two sites where explosives are being manufactured.

\subsubsection{Outside Quantity Distances}

Outside quantity distances are the distances separating the donor and the surrounding areas that are outside the storage or manufacturing zone of the donor. They are divided into two types (Kasemets, Natural Resources Canada, 2008):

- Public Traffic Route Distances: They are applied between a donor site and public traffic routes.

- Inhabited Building Distances: They are applied between a donor site and buildings or sites where members of the general public or persons not involved in explosives handling or work.

\subsection{Storage and transportation of hazardous materials}

\subsubsection{Existing Containments Systems (ISO containers)}

Existing storage systems also known as "ISO containers" are standardized reusable steel boxes designed for the storage or transportation of regular materials. Transport containers are classified in accordance with International Organization for Standardization (ISO) code and are manufactured in lengths of $5896 \mathrm{~mm}(20 \mathrm{ft})$ or12029 mm $(40 \mathrm{ft})$. The width and height of the containers are standard, $2350 \mathrm{~mm}$ and $2392 \mathrm{~mm}$ respectively. For most of the containers, the main frame is made of steel while the walls differ from one container to another. The wall of containers can be made of corrugated steel sheets, aluminum sheets and/or plywood with fibre- 
glass coating. The material type depends on the application or intended use of the container. Figure 10 shows the components of a typical shipping ISO container.
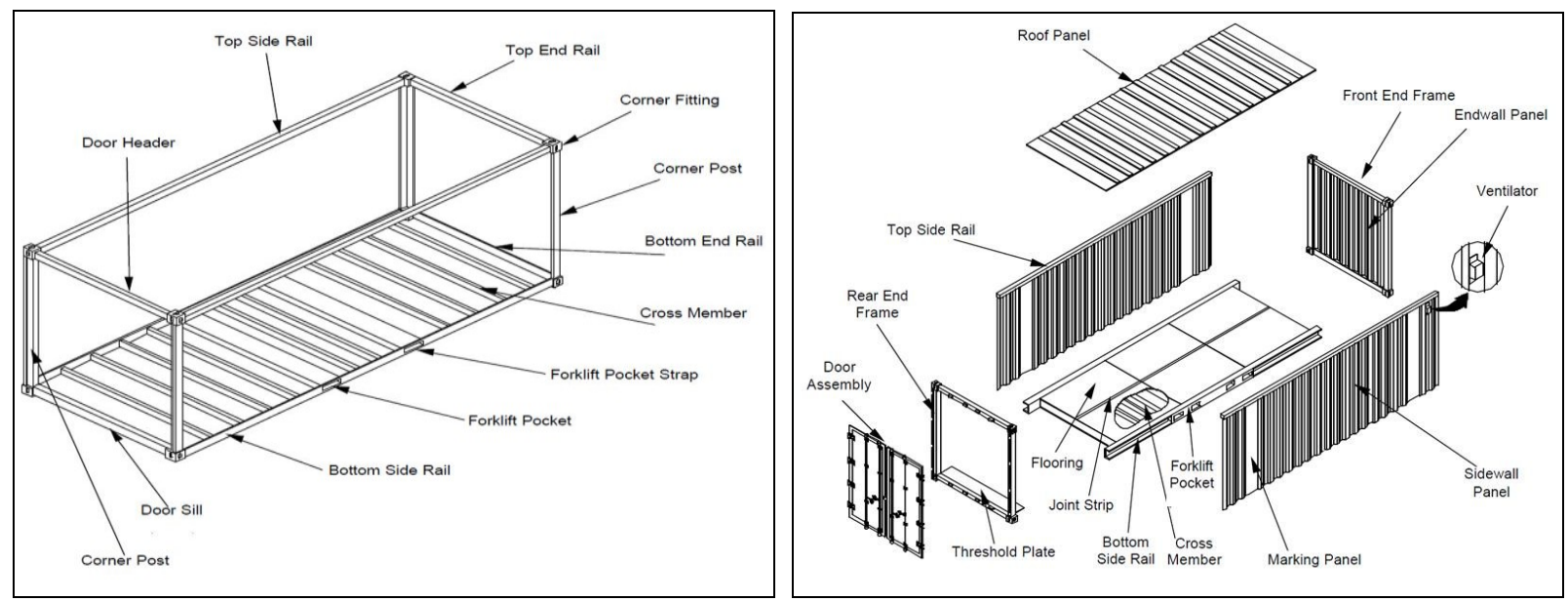

Figure 10: Typical Shipping Container Exploded View (Ecocontainerhome website)

Table 2 presents the internal dimensions as well as the maximum weight limits for $6 \mathrm{~m}$ (20-ft) and $12 \mathrm{~m}(40-\mathrm{ft})$ containers. These containers are used in the storage of regular materials, which do not have the risk of explosion.

Table 2: The standard 20 and 40 feet containers, (Shipping containers 24 website)

\begin{tabular}{|c|c|c|c|c|c|c|c|c|}
\hline \multicolumn{3}{|c|}{ Internal dimensions } & \multicolumn{2}{|c|}{ Door openings } & \multicolumn{3}{|c|}{ Weights } & Volume \\
\hline Length & Width & Height & Width & Height & $\begin{array}{c}\text { Max. } \\
\text { gross } \\
\text { wt. }\end{array}$ & $\begin{array}{c}\text { Tare } \\
\text { weight }\end{array}$ & $\begin{array}{c}\text { Max. } \\
\text { payload }\end{array}$ & $\left(\mathrm{m}^{3}\right)$ \\
\hline$(\mathrm{mm})$ & $(\mathrm{mm})$ & $(\mathrm{mm})$ & $(\mathrm{mm})$ & $(\mathrm{mm})$ & $(\mathrm{kg})$ & $(\mathrm{kg})$ & $(\mathrm{kg})$ & \\
\hline 5895 & 2350 & 2392 & 2340 & 2292 & 30480 & 2250 & 28230 & 33.2 \\
\hline 12029 & 2350 & 2392 & 2340 & 2292 & 30480 & 3780 & 6700 & 67.7 \\
\hline
\end{tabular}

The ISO containers shown in the table above are designed to transport and store regular materials but not hazardous materials. In case of internal explosion accidents, these containers are completely destroyed as was shown earlier in previous accidents. Studies are been carried out to investigate the possibility of having a strong containers that are able to withstand the blast load in case of the occurrence of an internal explosion and are light in weight to be used in the transportation activities. 


\subsubsection{Suppressive Shield}

Since the early 1900 's, several studies have been carried out to study the attenuation of internal explosion as well as the elimination of fragments. The first concept was using confined structures to eliminate the effects of internal explosions (Carre et al., 1970). These confined structures are still in use today. In order for these structures to be able to withstand an internal explosion, their wall section has to be thick and that makes them very heavy. Some companies such as Walkers and U.S explosive storage have developed steel magazines that can be used to contain blast loads as well as fragments. These magazines are made of high-strength steel with thick cross sections with a very heavy weight. Therefore, these magazines are used for the storage of hazardous materials but they are not economical to be used in transportation. Figure 11 shows an example for a magazine that is used to store explosives. However, the weight of this container was very heavy which makes it uneconomical and unpractical to be used in the transportation activities. Researchers are trying to achieve a structure design for magazines that could attenuate the blast load, contain fragments, light weight and cost less at the same time.

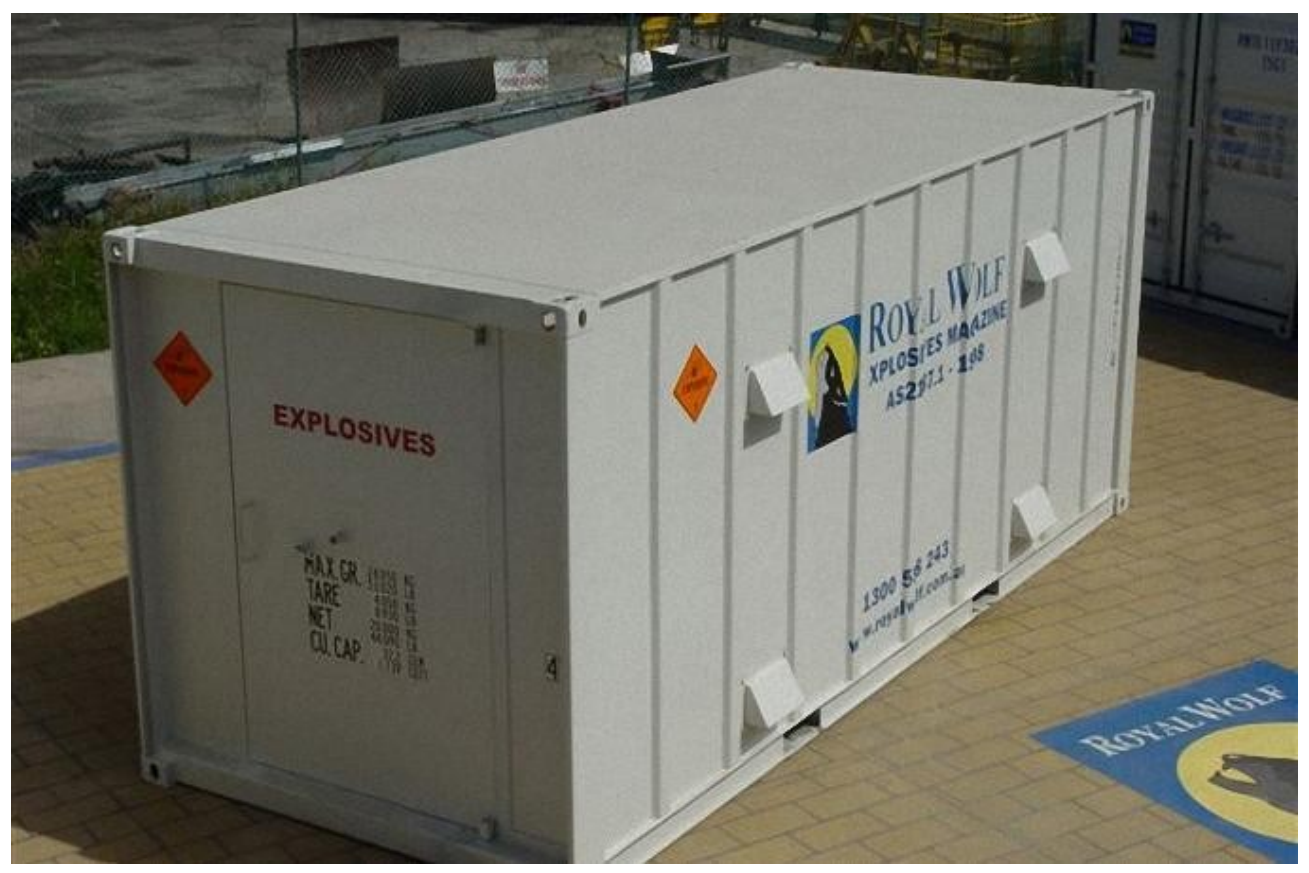

Figure 11: Explosive magazine (Royal Wolf website)

The challenge in designing a confined structure to contain internal explosion is due to the pressure buildup inside the structure from the gas products. In order to overcome this challenge, 
some openings within the section of the structure os required to allow a partial release of the internal pressure. This idea led researchers to study the possibility of using perforated containers (Figure 12). The perforated containers were able to release some of the pressure inside the containment structure and reduce the load on the side walls and roof of the structure. However, these openings created a direct path for the fragments to propagate outside the containment. Figure 12 shows an example for the developed perforated containments.

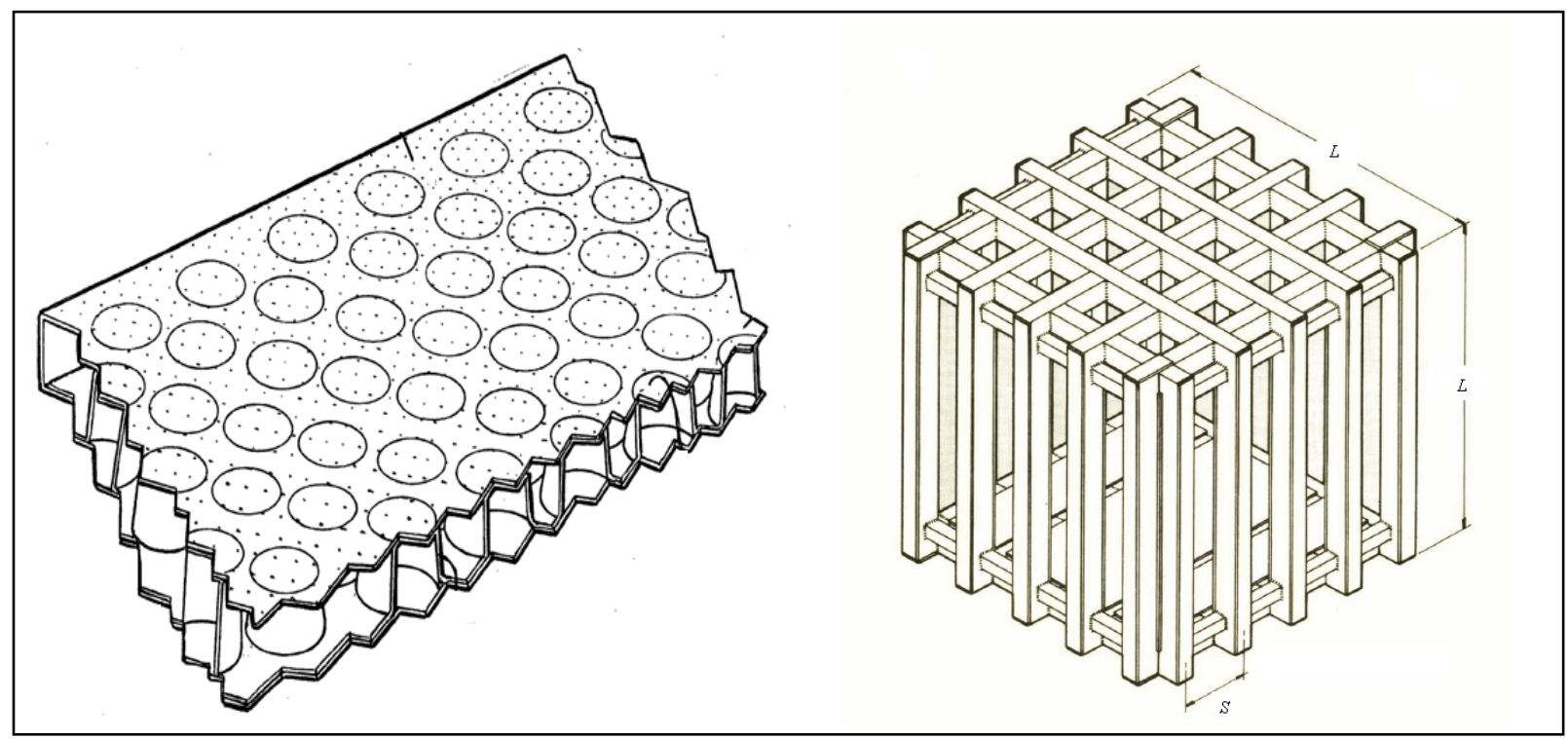

Figure 12: Perforated steel layer, (Palley et al., 2006)

In order to overcome the problem of having direct paths where the fragments can propagate outside the container, another perforated layer is added and arranged in a staggered order with the first layer (Palley et al., 2006). The two layers are spaced by a gap distance to allow the pressure waves to propagate through the structure but not the fragments. Figure 13 shows an example for the double layer vented section that was introduced to overcome the problem of having direct paths for fragments. 


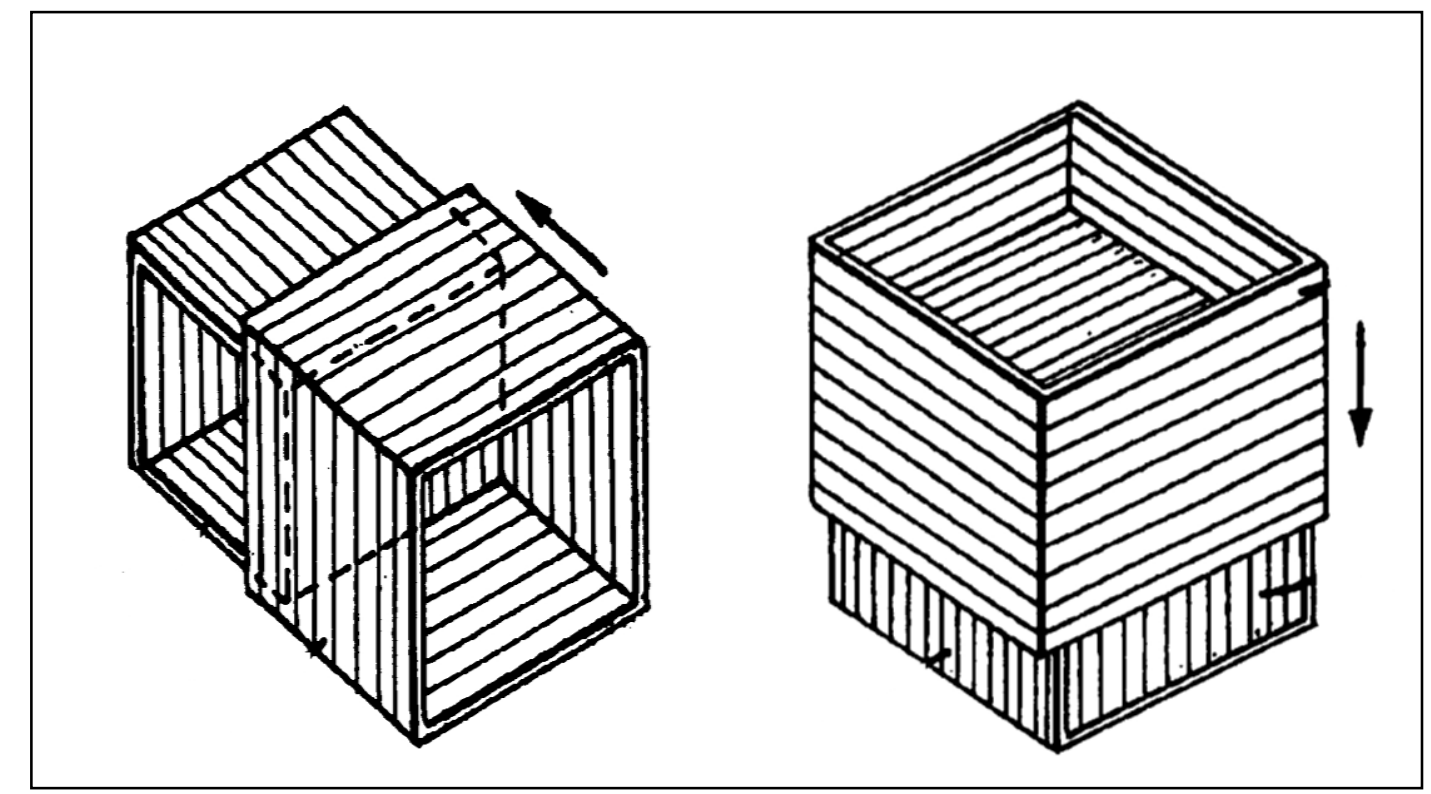

Figure 13: Double layer steel containments, (Palley et al., 2006)

According to the "USACE, 1977", vented suppressive shield is defined as a vented steel enclosure which controls or confines the hazardous blast, fragment, and flame effects of detonations and thus reduces the Q-D required around the containment structure (See Figure 14). The primary objective of using suppressive shields is to attenuate the blast pressure and eliminate fragments at the Q-D specified distance. The suppressive shields may also mitigate the effects of the fireball associated with explosion from the area surrounding the center of detonation. It is very challenging to design a steel section that can withstand the gas pressure due to an internal explosion especially for containers and magazines due to the limited distance between the charge and the walls of the container.

One technique used to resist the blast loads in suppressive shields is to allow release some of the internal pressure to the outside. For safety, the released pressure level at the specified Q-D should not exceed the maximum allowable to prevent injury to people and damage to the surrounding infrastructure systems. The presence of these vents in the containment structure allows hot high pressure gas from the explosion reaction to escape out of the container to a limited safe range and consequently affects both internal and external pressure (Kinney and Graham, 1985). 


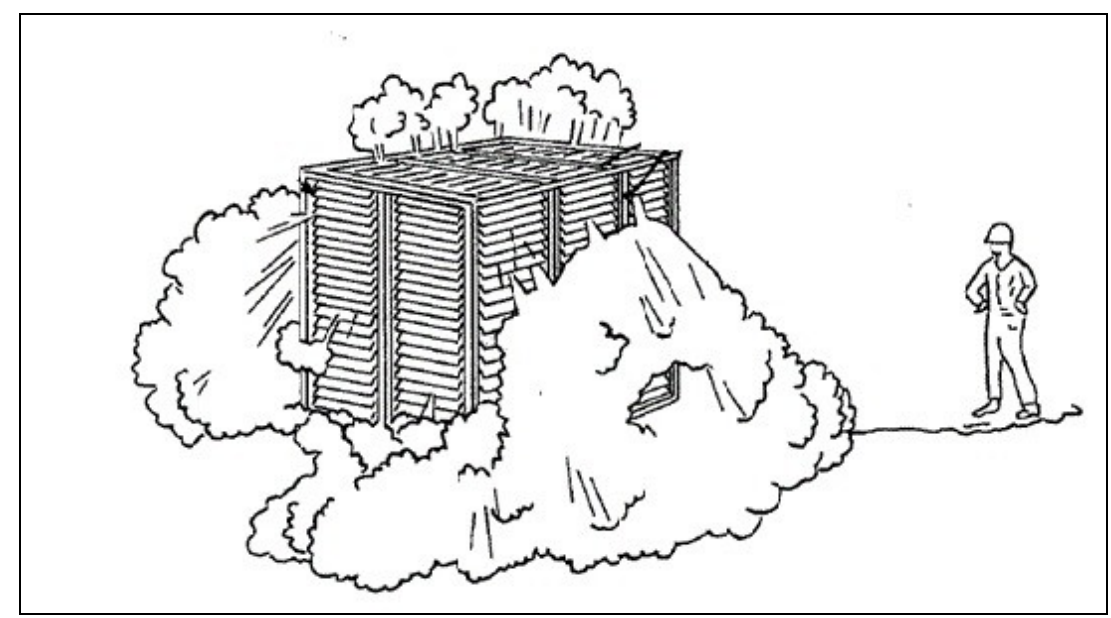

Figure 14: Vented Suppressive Shield containment, (King et. al, 1981)

The steel section of the VSS enclosure is designed depending on the function for which the suppressive shield could be used. VSS can be used in a variety of areas to improve personnel safety and facility protection and they include:

1. Embassy Security including the perimeter, internal walls, VSS rooms, and VSS protection of air ventilation ducts.

2. Airport Security where VSS containers can be used for luggage and unattended packages suspected of containing explosives.

3. Protection of Weapon System Components: a protective container around rocket propellant.

4. Storage of Explosives: walls and magazine storage VSS units for reduction of quantity-distance requirements.

5. Manufacture of Propellants: barriers to protect personnel and equipment and reduction of quantity-distance requirements.

6. Explosive Ordinance Disposal (EOD): VSS enclosures for the safe disposal of pipe bombs, bulk explosives, and other explosive devices.

7. Oil Platforms to protect personnel and equipment from flame, blast and fragments in a blow-out or fire. 


\subsection{Vent Area Ratio}

The vent area ratio in VSS structures is defined as the ratio between the vent areas $\left(A_{\text {vent }}\right)$ to the total area $\left(A_{\text {total }}\right)$ of the structure as shown in Equation 1. The role of the vents in the containment structure is to prevent the gas pressure from building up and increasing the pressure inside the containment structure. For a very small percentage of openings or in unvented containers, the duration of the gas pressures is very long in comparison to the fundamental periods of the structure's elements and, therefore, may be considered as a long duration load (static load). A typical pressure-time record at a point on the interior surface of a partially vented chamber was shown earlier in Figure 4. The high peaks are the multiple reflections associated with shock pressures. The gas pressure, denoted as $p_{g}$, is used as the basis for design and is a function of the charge weight and the contained net volume of the chamber.

$$
\alpha_{i}=\frac{A_{v e n t}}{A_{\text {total }}}
$$

Equation 1

The suppressive shield panels can be made of angles, zees, louvers or interlocked Ibeams and as a result the calculation of the vent area $\left(A_{v e n t}\right)$ changes from one geometry to another. For example, the angles in nested configuration (Figure 15) will require different procedures for calculating the vent ratio than for a side-by-side configuration (Figure 16) as shown in the equations below the figures. Generally, the vent area calculation of any section depends on the number of openings, length of exposed element, length and width of the suppressive shield panel, (U.S. Army Corps of Engineering, 1977). 


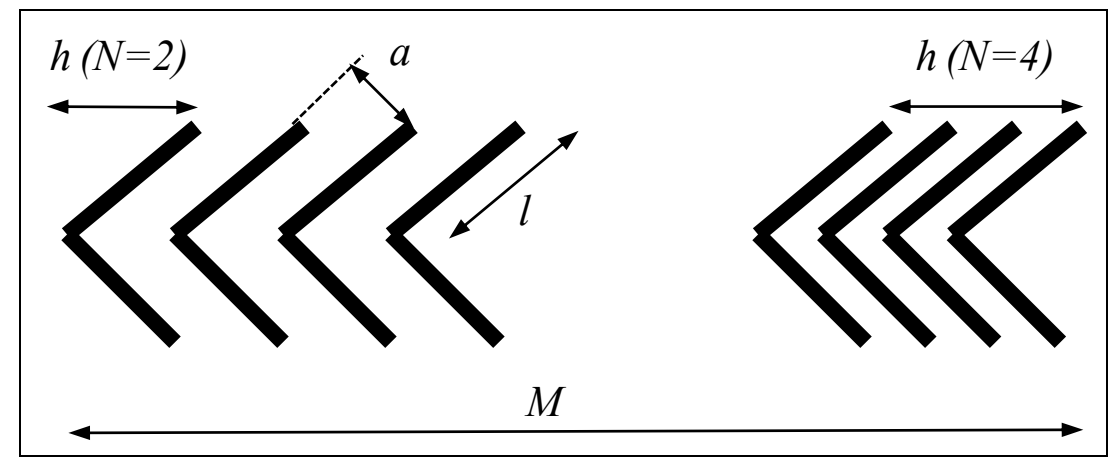

Figure 15: Definition of Vent Area Ratios for Nested angles configuration, (Baker et al. 1982)

$$
A_{\text {vent }}=\frac{a}{h} / N
$$

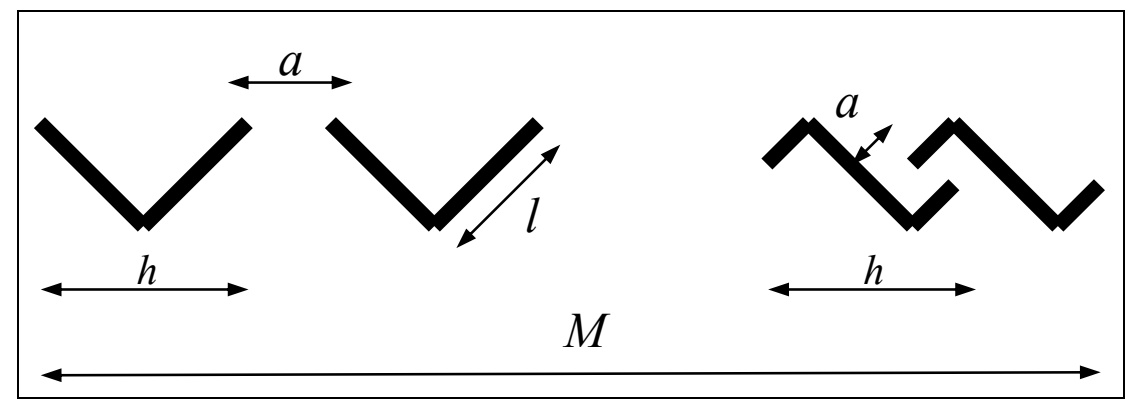

Figure 16: Definition of Vent Area Ratios for Side-by-side angles configuration, (Baker et al. 1982)

$$
A_{\text {vent }}=\frac{a}{h}
$$

Where, $n$ is number of openings, $l$ is the length of exposed element, $N=2$ for one opening per projected width $(h), \mathrm{N}=4$, for two openings or more per projected width $(h), A_{v e n t}$ is the Area of vents. When the vent area $\left(A_{\text {vent }}\right)$ is calculated, the vent area ratio $(\alpha)$ is then calculated using Equation 1. The term $A_{\text {total }}$ in Equation 1 in this case is substituted by $A_{\text {wall }}$ (Area of wall of VSS container) $=L \times M$, where $L=$ length of wall, $M=$ width of wall.

As mentioned earlier, suppressive shield containment structures can be made with more than one layer of shield arranged in a staggered way in order to prevent the direct paths of fragments. These sections are referred to as multilayer VSS system. Equation 1 is used to calculate the vent area ratio of a single layer of VSS section. However, for the multi-layer system, the vent area ratio of each layer is calculated using Equation 1 and then the effective vent area ratio of the entire section is estimated using Equation 2 (Cox et al., 1978). 


$$
\frac{1}{\alpha_{e}}=\sum_{i=1}^{m} \frac{1}{\alpha_{i}}
$$

Equation 2

Where, $\alpha_{e}$ is the effective vent area ratio (for the multiple layer VSS section), $\alpha_{i}$ is Single layer vent area ratio of each layer of the section and $m$ is number of layers

\subsection{Calculation of Pressure inside enclosed structures}

In order to design an element that is subjected to blast load, the reflected pressure $\left(P_{r}\right)$ needs to be computed. If the angle of incidence is zero, then the blast wave is assumed to be normally reflected. The incident and reflected pressure profile can be obtained from Friedlander waveform equation expressed in Equation 3 and Equation 4, respectively (Needham, 2010).

$$
\begin{gathered}
P(t)=P_{s o}\left(1-\frac{t}{t_{d}}\right) e^{\frac{-b}{t_{d}}} \\
P_{r}(t)=P_{r}\left(1-\frac{t}{t_{d}}\right) e^{\frac{-b}{t_{d}}}
\end{gathered}
$$

Equation 3

Equation 4

Where $P_{s o}$ is the incident peak pressure value at the point of interest, $t$ is the time where the pressure is tracked, $t_{d}$ is the positive phase duration, $b$ is the decay coefficient that measures the rate of decay of the blast pressure and $P_{r}$ is the peak reflected pressure value at the point of interest.

Another parameter that needs to be calculated is the impulse. Lacking accurate prediction methods, the reflected impulse can be estimated by assuming similarity between the time histories of side-on overpressures and normally reflected overpressure. The ratio of the reflected impulse $\left(I_{r}\right)$ to the incident impulse $\left(I_{s o}\right)$ is assumed to be the same as the ratio of reflected $\left(P_{r}\right)$ to incident pressure $\left(P_{s o}\right)$ as shown in Equation 5 (Braimah, 2012). 


$$
\frac{I_{r}}{I_{s o}}=\frac{P_{r}}{P_{s o}}
$$

\section{Equation 5}

As mentioned earlier, when an explosion occurs within a confined structure, the shock wave from the explosion hits the wall of the structure. The wave then reflects at the walls of the container. All the reflected waves from all the sides of the container then meet and reflect again towards the walls of the container forming a second shock wave. Subsequence shock waves were subsized due to loss of energy. This keeps happening until the blast load dissipates. Baker et al. (1983) introduced a simplified relationship between the consecutive shock waves. Figure 17 shows the simplified pressure profile for an internal explosion in a confined structure. It is assumed that the first three shock waves can be taken into consideration but after that, the shock waves are not assumed to be significant. The first shock wave is calculated as a regular blast shock wave since there is no difference between the shock wave hitting a target from inside the container or outside. While for the second and third shock waves, a ratio was introduced relative to the first shock wave. The ratio between the first and second is assumed to be $2: 1$ and the ratio between the third and the second is $2: 1$ as well. This means that the ratio between the first and the third is $4: 1$. The arrival time $\left(t_{a}\right)$ is the time that takes the blast wave to hit the side of the container. The blast load is reflected back until it collides with all the reflected waves from all sides of the container at the center of the container then reflects again towards the walls of the container. The time $\left(3 t_{a}\right)$ is the time the shock wave takes to reflect a second time at the side of the container. The time each shock lasts on the side walls $\left(t_{r}\right)$ is assumed to be $\left(2 t_{a}\right)$. 


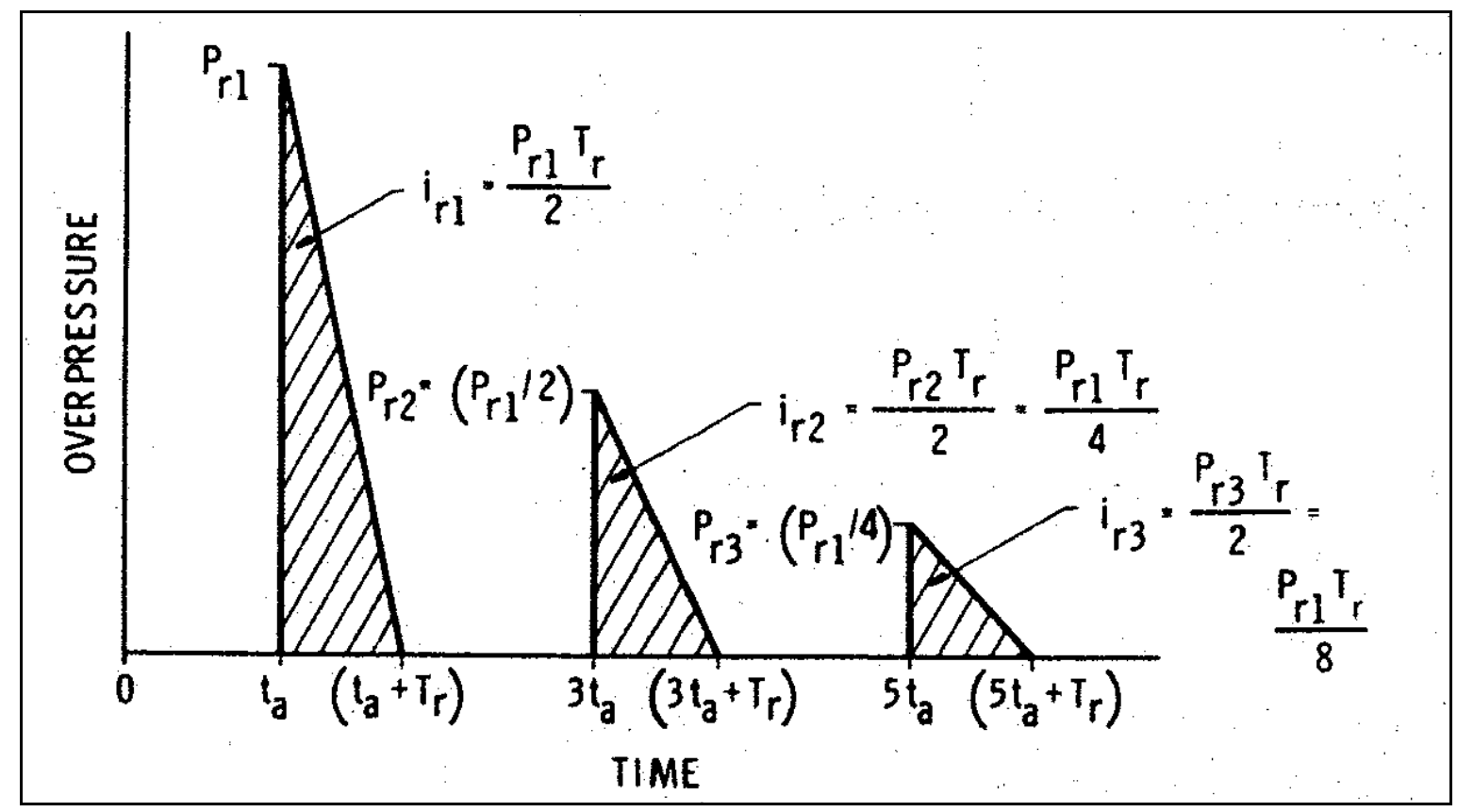

Figure 17: Simplified internal blast pressure (Baker et al., 1983)

\subsection{Previous Work on Suppressive Shields}

Suppressive shields are mainly used in the fields related to explosives manufacture, storage and transportation. The first blast suppressive shield section was introduced in 1976 (King et al., 1981) and was assigned a patent until it was released to the public in 1981. The blast suppressive shield was primarily designed to eliminate the hazards associated with manufacture, storage and transportation of explosives, propellants, and pyrotechnics. The suppressive shield section had air vents to allow the blast pressure to propagate outside the container. In his study, some experimental tests were carried out to investigate the performance of the blast suppressive shield section in the attenuation of blast loads. One of the tests that were carried out was a comparison between storing $4.2 \mathrm{~kg}$ white phosphorus mortar shells stored in regular storage magazine and others stored in the blast suppressive shield. It was found that when the white phosphorous was detonated, fragments were scattered within an area with diameter of approximately 300 meters for the regular storage magazine. While for the blast suppression shield, the fragments were contained and the resultant fireball size was also reduced. The tests were repeated for a wood storage magazine with and without the blast suppression shield. It was found that the wooden magazine without the shield was completely destroyed and this ensures the need for the suppressive shields in the case of wooden containers. 
In the same study, a test was conducted on a portable suppressive shield container to transport about five pounds of high explosive through densely populated areas. The results from this test showed complete elimination of fragments and blast overpressure. The most important conclusion from this study was that for the proposed containers system, the optimum vent area ratio varied from 0.25 to 0.6 of the overall area (King et al. 1981).

Kivity et al (1993) studied the response of structures to internal explosions. The authors studied the concept of the presence of vents in the structure and how they affect the response of the structure due to the internal blast load. The authors studied the effect of the vent area ratio for different kinds of structures; rigid structures such as concrete and thin-walled structures such as steel cylindrical container. In this study, MSC/DYTRAN, a finite element program, was used to simulate the vented structure with an internal explosion. For both cases, rigid and thin walled structures, the presence of a vent in the structure section released some of the internal pressure inside the structure and hence reduced the resultant damage to the structure.

In 1997, a patent was released by the United States (Crane et al., 1997). The patent was known as "High-Energy-Absorbing Enclosure for Internal Explosion Containment". The objective of this invention was to provide a structural enclosure which effectively contains an explosion within that structure. The containment structure was designed to contain both the blast pressure as well as the fragments. The section of the containment structure was made of three layers; and was named as "sandwich-like" section. The internal and external layers were made of fiber-reinforced material while the material between them was a foam material. The foam layer acted as a damper to absorb the pressure due to the internal explosion and hence reduce the stresses on the outer materials. For the angles at the corners, it was designed in such a way to have semi-spherical vertices to reduce the concentration of the stresses at the corners. The introduced containment structure was able to contain the blast load and the fragments of small charges, while for bigger charges the failure was found to be at the corners of the structure. Figure 18 shows the container before and after the explosion. 


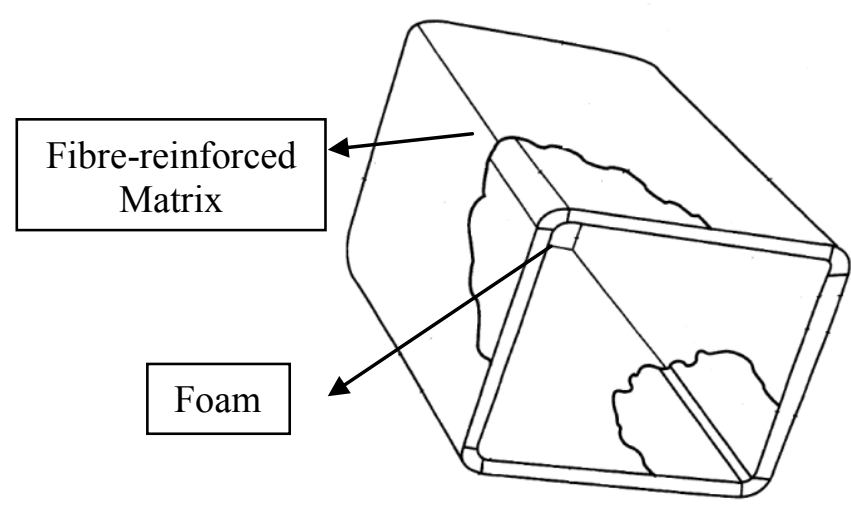

(a) Before internal explosion

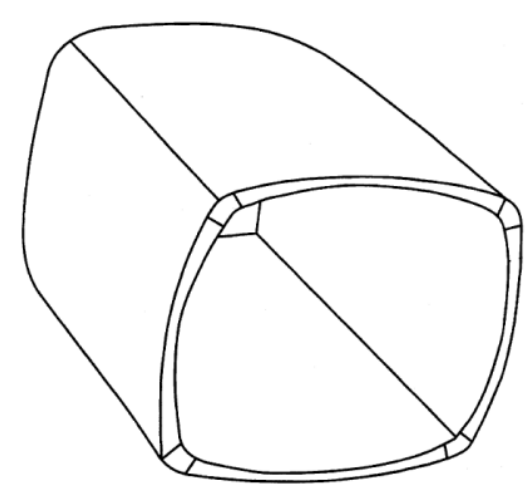

(b) After internal explosion

Figure 18: Deformation in the container (Crane et al. 1997)

One important study on the suppressive shields was completed by ElShafey (2008). The main objective of this study was to improve the safety and security in the transport and storage of explosives. The objective was achieved by testing different sections of suppressive shields with different vent areas ratios and studying the blast waves' propagation through the suppressive shield without the failure of the shields. In this study some experimental tests were carried out. Different sections with different configurations of steel angles were exposed to different explosive charges and the pressure recorded inside and outside the container. The experimental work was verified by simulation of the problem using AUTODYN software that showed good correlation with the experimental results. Four sections were proposed in the study and are shown in Figure 19. 


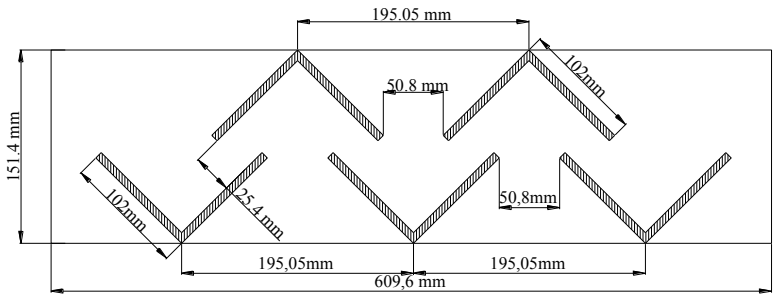

(SSP-A)

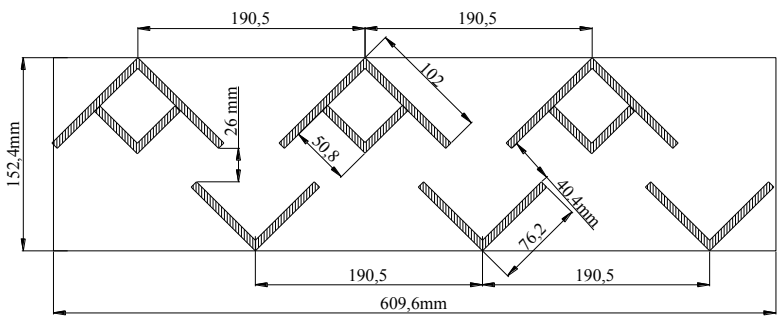

(SSP-C)

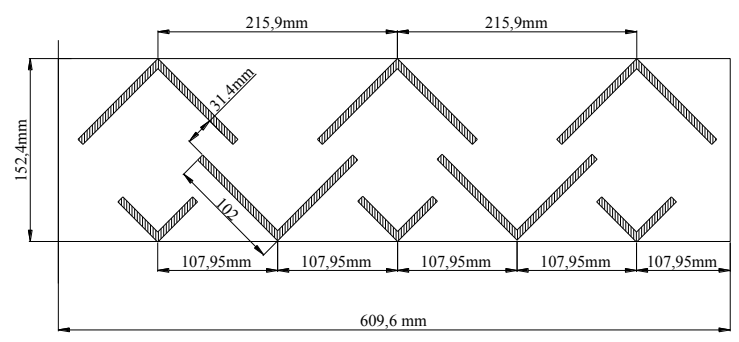

(SSP-B)

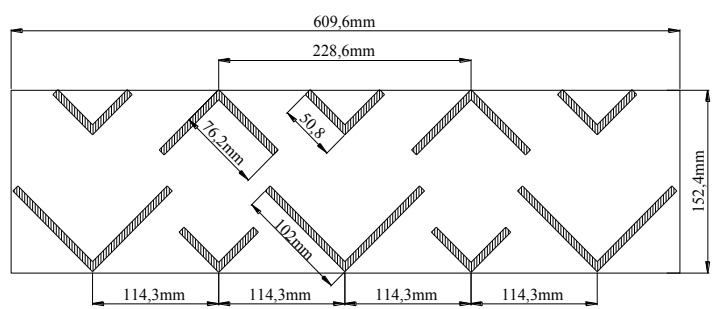

(SSP-D)

Figure 19: Suppressive Shield Panels studied (EIShafey, 2009)

ElShafey (2008) introduced different geometric configuration that reduced the pressure outside the container. The results showed that the two configurations (SSP-A) and (SSP-B) shown in Figure 19 showed the best performance compared to the others. The used configurations were a combination of different size steel angles arranged in a staggered arrangement. These sections were labelled "double angle configuration" by the author. The blast attenuation of the four studied sections was almost equal, so the governing factor was the weight of the section. As a result, configuration (SSP-A) was found to be the best configuration between the studied configurations. In this study, the author did not study the welded connection between the suppressive shield elements and the main frame of the container although the maximum stresses along the suppressive shield were noticed to be at the ends of the suppressive shield section at the welding locations.

In the same research, the authors studied the effects of adding aluminum foam (AF) on the interior face of the VSS walls (Braimah et al., 2012) using both experimental testing as well as modelling using AUTODYN software. The role of the aluminum foam was to act as a flexible layer in front of the rigid steel layer. The presence of the flexible layer (aluminum) was able to absorb a portion of the energy from the explosion and thus reducing the load that affects the steel angles forming the side wall of the VSS section. 
In general, when the SSPs were lined with AF sheets, percent attenuation of peak incident pressure of up to $80 \%$ and percent attenuation in incident impulse of up to $79 \%$ were achieved compared to $66 \%$ and $76 \%$ respectively. These percentages demonstrate the ability of AF sheets to dissipate a large amount of energy under blast loading.

Elshafey (2008) also observed that the failure of the VSS containers occurs at the corners of the container. His explanation to this phenomenon was due to the concentration of the blast waves at the internal vertices of the container. The concentration of the waves at the corners increases the stresses at the corners and thus failure occurs.

\subsection{Finite Element Modelling (FEM)}

In studying how structures resist blast loads, full scale blast tests should be carried out. Full scale blast tests of real structures are rare because of their size, complexity and above all, the costs required for their execution. So another way to study the effect of blast loads on structures is using Finite Element methods (FEM). FEM has proven reliable results in the simulation of the blast problems and their output results could be used instead of carrying out experimental work. In the current study, the effect of blast loading on VSS containers will be studied using AUTODYN software that is based on Computational Fluid Dynamics (CFD). The following section addresses a brief introduction about Computational Fluid Dynamics (CFD) codes and the governing equations that are used in the CFD programs.

\subsubsection{ANSYS AUTODYN}

Blast loads are different than other types of loading due to their very high speed and magnitude. Also in blast loading, there is an interaction between the compressed layer forming the shock wave and the target. In order to simulate this kind of interaction, hydrodynamic codes known as Hydrocodes have been used. Hydrocodes originally performs calculations by assuming hydrodynamic behaviour in the materials, and therefore ignoring material strength, which is the origin of the term "Hydrocode". This method was used because the pressures generated by experiments often greatly surpassed the strength of the materials [Zukas, 2004]. Also, while many of the calculations performed by hydrocodes could be done by hand or even with the use of a calculator, the huge number of calculations involved in even simple problems makes the use of powerful computers invaluable. 
Computational Fluid Dynamics (CFD) programs are known as the "modern Hydrocodes". CFD can make use of a variety of methods to model different material behaviours. In addition to their use in blast modeling, hydrocodes have been used to evaluate structures for aircraft impacts, to simulate vehicle crashes and even design sports equipment [Zukas, 2004]. Blast loads are characterized by their fast loading rate and can be expressed by a function of time. These kinds of problems are known as time transient problems where time is an important factor when computing the blast pressure, so the numerical methods used for the simulation of the blast problem are typically based upon a finite difference, finite volume, or finite element method utilizing explicit time integration.

ANSYS AUTODYN is an explicit analysis tool for modeling nonlinear dynamics of solids, fluids, gases and their interaction especially for large strain and deformations. With a fully integrated, easy to use graphical interface allowing set up, running, and post processing of problems (AUTODYN Manual, Century Dynamics Inc., 2005). AUTODYN software incorporates a number of different, widely used numerical techniques. Each of these techniques has certain advantages and disadvantages. No single method can handle all regimes of a typical problem. The challenge is to apply these techniques in the most efficient and accurate fashion, in accordance with the demands of the problem, in order to find an optimal solution. The various techniques used in the AUTODYN software can generally be characterized as follows (Birnbaum, 1999):

- Lagrange: the numerical mesh moves and distorts with the material motion (Figure 20).

- Euler: the numerical mesh is fixed with the material flowing through it (Figure 21).

- ALE: Arbitrary Lagrange Euler which provides a type of automatic rezoning, overcoming some of the limitations of both Euler and Lagrange.

- Structural elements: Thin shells and other structural members are treated. 


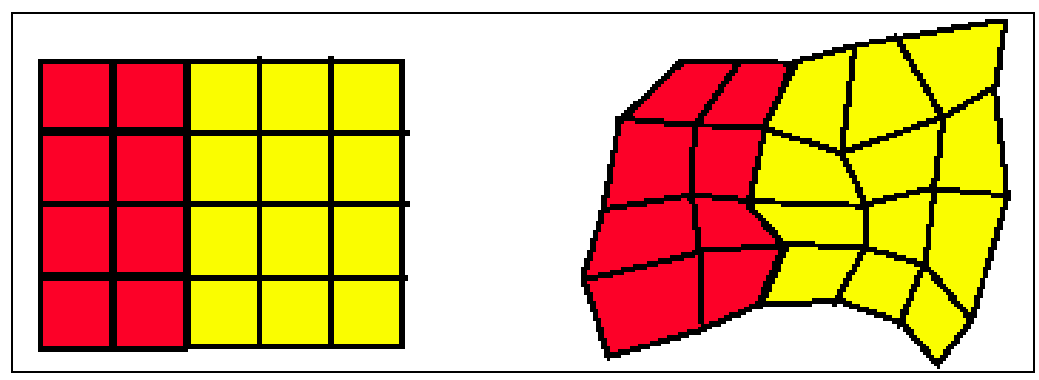

Figure 20: Lagrange mesh, (Birnbaum, 1999)

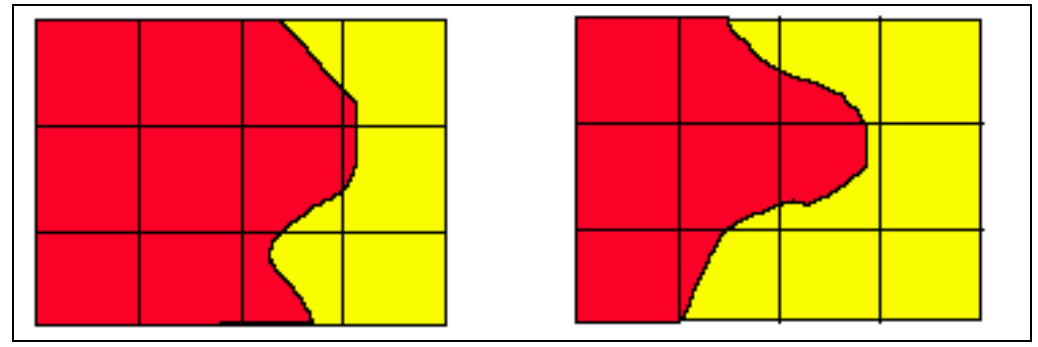

Figure 21: Euler mesh, (Birnbaum, 1999)

\subsubsection{Governing equations}

AUTODYN is mainly based on three conservation principals. The first principle is the law of conservation of mass. It states that the mass of a closed system remains constant, regardless of the processes acting inside the system. An equivalent statement states that matter cannot be created or destroyed, although it may be rearranged as long as it is in a closed system. This means that for any chemical process in a closed system, the mass of the reactants must be equal to the mass of the products. This is also the main principal behind the first law of thermodynamics.

The second principle is the law of conservation of momentum. The law of conservation of momentum is a fundamental law of nature, and it states that the total momentum of a closed system of objects that has no interactions with external agents is constant. One of the consequences of this is that the center of mass of any system of objects will always continue with the same velocity unless acted on by a force from outside the system.

The third principle is the law of conservation of energy. In physics, the law of conservation of energy states that the total amount of energy in an isolated system is constant. A consequence of this law is that energy cannot be created or destroyed. The only thing that can 
happen with energy in an isolated system is that it can change its form. As in explosions, the kinetic energy can become thermal or sound energy. Because energy is associated with mass in Einstein's theory of relativity, the conservation of energy also implies the conservation of mass in isolated systems.

In AUTODYN software, the three principals (governing equations) are satisfied at every time step. In addition, a constitutive law is required for material modeling that links stress to deformation and internal energy. This system of differential equation is solved in AUTODYN using a combination of finite volume, finite element and mesh free solver technologies. The solution methodology is based on explicit time integration (Fišerová, 2006). Fluid dynamics as a scientific discipline starts with the Euler equations expressing conservation of mass, momentum and total energy as a system of partial differential equations. The main variables involved in the Euler equation are the pressure, density, Cartesian velocity components, total energy, and total enthalpy, denoted by $P, \rho, u, v, E$, and $H$, respectively. The governing equations that describe the conservation of mass, momentum and energy for the evolution of a non-viscous, ideal fluid can be written on integral or differential form, based on Eulerian or Lagrange description. Equation 6, Equation 7 and Equation 8 show the differential equations for the case of an Eulerian description in differential form.

$$
\begin{array}{cc}
\frac{\partial \rho}{\partial t}+\nabla \cdot(\rho u)=0 & \text { Equation } 6 \\
\frac{\partial \rho u}{\partial t}+\nabla \cdot(u \otimes(\rho u))+\nabla P=0 & \text { Equation } 7 \\
\frac{\partial E}{\partial t}+\nabla \cdot(u(E+P))=0 & \\
\hline
\end{array}
$$

where $\nabla$ (Nabla) means the gradient. The gradient is the vector field whose components are the partial derivatives of a function $\mathrm{F}$ given by $(\mathrm{df} / \mathrm{dx}, \mathrm{df} / \mathrm{dy}) . \rho$ is the fluid mass density, $u$ is the fluid velocity vector, with components $u$, $v$, and $w, E=\rho e+1 / 2 \rho\left(u^{2}+v^{2}+w^{2}\right)$ is the total energy per unit volume, with $e$ being the internal energy per unit mass for the fluid, and $P$ is the pressure. 
The previous equations can be written for the Lagrangian case by using Lagrangian derivative as shown in Equation 9.

$$
D / D t=\partial / \partial t+v \cdot \nabla
$$

\section{Equation 9}

Equation 10, Equation 11 and Equation 12 show the governing equations using the Lagrangian derivative.

$$
\begin{gathered}
\frac{D \rho}{D t}=-\rho(\nabla \cdot v) \\
\frac{D v}{D t}=\frac{\nabla \cdot P}{\rho} \\
\frac{D e}{D t}=-\frac{P}{\rho}(\nabla \cdot v)
\end{gathered}
$$

Equation 10

Equation 11

Equation 12

The above equations are expressed in conservation form, as this format emphasizes their physical origins and is often the most convenient form for computational fluid dynamics simulations. For ideal gas, the following relations are used:

$$
\begin{gathered}
E=\frac{P}{(\gamma-1)}+\frac{1}{2}\left(u^{2}+v^{2}\right) \\
H=E+\frac{P}{\rho}
\end{gathered}
$$

Equation 13

Equation 14

Where $\gamma$ is the ratio of specific heats 


\subsubsection{Material model}

Material properties of a given metal changes depending on the kind of load applied on the specimen. Under dynamic loading such as blast loads, high strain rate gradients are initiated in the material that are accompanied by the change in temperature due to the adiabatic character of high rate deformation processes. In order to simulate the behavior under dynamic loading, the material model should be able to take into account the effect of wide ranges of strain rate and temperature (El-Magd, 1994). The material models used in AUTODYN can be broken down into three categories. These categories are the equation of state, which is used in providing volumetric stress or pressure. The strength models, that determines the deviatoric stresses based on Hooke's Law and a plastic yield criteria. The last is the failure model, which provides a criterion for determining if a material has failed and no longer has strength (Kloster, 2001).

\subsubsection{Equation of state}

The equation of state is a thermodynamic equation that is used to describe the material status and material properties using relations between various variables to define each material. The general form of the equation of state (EOS) for pressure is function of the local density $(\rho)$ and the local specific internal energy $(e)$ of material. For static problems, the material behavior is expressed linearly without defining the EOS. However in case of dynamic loading such as blast loading, the non-linear material behavior must be taken into consideration. The general equation of state can be expressed in Equation 15 (AUTODYN user's manual, 2005):

$$
P=f(\rho, e)
$$

\section{Equation 15}

\section{1) Ideal gas}

The ideal gas equation of state relates the pressure $(P)$ to the specific internal energy $(e)$, such that:

$$
P=(\gamma-1) \rho \cdot e
$$

\section{Equation 16}

where $\gamma$ is the adiabatic constant (ratio of specific heats) and $\rho$ is the density. 


\section{2) Linear equation of state}

For the steel material, the linear equation of state was used which is based on Hooke's law type relationship between the pressure $(P)$ and the volume change as:

$$
P=K \mu
$$

\section{Equation 17}

where $\mathrm{K}$ is the bulk modulus of the material, $\mu=\left(\frac{\rho}{\rho_{0}}-1\right)$ the compressed amount of volume, $\rho_{0}$ is the reference density.

\section{3) JWL equation of state}

The JWL equation of state defines the explosive shock wave pressure (p) as a function of the specific volume (V) and the specific internal energy (e), such that (Lee et al., 1973):

$$
P=\frac{\omega E}{V}+A\left(1-\frac{\omega}{R_{1} V}\right)+B\left(1-\frac{\omega}{R_{2} V}\right)
$$

Equation 18

where $A, B, R_{1}$ and $R_{2}$ are constants, $E$ is the internal energy, $\omega$ is adiabatic constant and $V$ is specific volume

\subsubsection{Strength models}

The main purpose of the strength models is to determine when a material yields. Because AUTODYN deals with loads of high magnitude and speed such as blast loads, large deformations are expected for the elements. The most commonly used strength model for steel material subjected to high strain-rates, large deformation, and high temperature is the JohnsonCook model.

\section{1) Johnson-Cook model}

The Johnson-Cook model is basically an empirical model that incorporates strain rate and temperature dependency on strength (Rajendran, 1990). The model was developed using results obtained from previous experimental work carried on a variety of metals (Hancock and Mackenzie, 1976). The model developed an experimental relationship that states the influences 
of temperature, strain and strain rate. This relationship has many applications in numerical simulation of material behaviour. The Johnson-Cook model has been demonstrated to provide realistic solutions to hydrocode predictions of a very broad class of applications that is related to extreme dynamic events such as impact, penetration, and explosive acceleration of metals. The success of the model is due to its ability to incorporate the overall effects of temperature and strain rate as observed from the dynamic and quasi-static experimental data (Rajendran, 1990). The Johnson-Cook failure model is used mainly to model the ductile failure of materials experiencing large pressures, strain rates and temperatures. Mathematically, this model is expressed as:

$$
\frac{1}{2}\left(\sigma_{1}-\sigma_{2}\right)^{2}+\left(\sigma_{2}-\sigma_{3}\right)^{2}+\left(\sigma_{3}-\sigma_{1}\right)^{2} \leq \sigma_{y}^{2}
$$

Equation 19

The stresses can be expressed in the following equation (Majzoobi et al., 2006):

$$
\bar{\sigma}=\left(\sigma_{0}+B \varepsilon_{p}{ }^{n}\right)\left(1+C \ln \frac{\dot{\varepsilon}}{\dot{\varepsilon}_{0}}\right)\left(1-\left(T^{*}\right)^{m}\right)
$$

Equation 20

Where $\varepsilon_{p}$ is the effective plastic strain, $\sigma_{0}, B, C, n$, and $m$ are five material constants that can be obtained from experiments. $\sigma_{0}$ is the yield strength; $B$ is the strain hardening coefficient; $n$ is the work hardening exponent; $C$ is strain rate coefficient, and $m$ is the thermal softening exponent. $\dot{\varepsilon}$ and $\dot{\varepsilon}_{0}$ are the current and reference strain rates. $T^{*}$ is the homologous temperature and is expressed as:

$$
T^{*}=\frac{T-T_{r}}{T_{m}-T_{r}}
$$

\section{Equation 21}

where $T_{r}$ and $T_{m}$ are room and material melting temperatures, respectively.

It can be found from Equation 20 that the first term at the right hand side of the equation shows the relation between semi-static stress and strain at the room temperature. The second term states the effect of strain rate and the last term illustrates the effect of temperature. 


\section{2) Failure model (Plastic Strain)}

Plastic strain failure model is considered one of the simplest models to define the failure of a material. It states that failure of a material element occurs when the effective plastic strain exceeds a specified value. Once this plastic limit is reached, failure occurs and the stresses are set to be zero and consequently the element can't sustain any shear stress beyond this point.

\section{3) Erosion model}

During simulations some Lagrangian element may experience large deformations leading to distortions. These distortions may slow the calculations time so it is recommended to remove those distorted cells since they no more offer structural resistance.

\subsubsection{Processor Coupling}

\subsubsection{Euler - Lagrange}

As mentioned before, two mesh techniques are used in AUTODYN to simulate the effect of blast loads on structural elements, Euler and Lagrange techniques. An interaction between the two different sub grids should take place in order to simulate the effect of the explosion on the structural elements. When the Lagrange sub grids are exposed to loading, the Lagrange sub grid moves and thus some Eulerian sub grids are covered or uncovered. The gap between the Euler and Lagrange subgrids defines the gap that the Lagrange sub grids are allowed to move or deform within the Eulerian ones.

\subsubsection{Lagrange-Lagrange}

When different Lagrange parts interact with each other, they need to be joined together in a way that makes them act as one element. An interaction gap should be defined in the AUTODYN model to make sure that the interacting nodes from two parts act together as one node. This gap depends on the mesh size of the Lagrange parts and how close the interactive nodes are with respect to each other. All the Lagrange elements should be taken into consideration when the interaction gap is defined. The interaction gap is calculated automatically by the software; however, if the gap size between the interactive nodes is bigger than mesh size 
of the element, the mesh size should be refined (Interaction tutorial - Century Dynamics Inc., 2005).

\subsection{Summary}

From the literature review, it was found that there are not enough available studies regarding the design of vented suppressive shields containers. Most of the available VSS containers have been designed based on experimental studies or previous accidents that make these VSS containers not economically designed. All the available containment structures that are used for the storage of hazardous materials are over designed and for that reason they are very heavy that makes them non transportable.

Although AUTODYN is a good computational tool to study the effect of blast loads on structures as well as studying the structural response of the elements subjected to blast loads, it is an expensive CFD package and it requires special experience and training to be efficiently used. Another disadvantage of AUTODYN is that it doesn't provide structural outputs; such as the reactions and rotations at the supports.

The main objective of the ongoing research is to present an independent simple design method that can be used to study the effect of explosions on VSS sections. This method will be able to evaluate the performance of different VSS sections as well as predicting the pressure profile on the side wall of the VSS containers. The design approach was also able to predict the deflections of steel sections due to the applied blast loads and determine the reactions and rotations at the supports that were used to design the connection between the side wall and the main frame of the container. This design approach helped in obtaining a strong, light weight structure that is able to attenuate internal blast loads and eliminate fragments. 


\section{Chapter 3: $\quad$ Numerical modeling}

\subsection{Introduction}

This chapter presents a detailed description of the 2D and 3D AUTODYN models that were developed in the research program. The 2D AUTODYN models were used to study the interaction between the blast wave and the suppressive shield elements as well as the attenuation of peak blast pressure and impulse outside Vented Suppressive shield (VSS) container. On the other hand, 3D AUTODYN models were modelled to study the structural response of the VSS containers due to internal explosion. The chapter also introduces the material properties of the structural steel elements and air medium in which the blast wave propagates, boundary conditions assigned in the models, and failure modes of the elements that were used in this study. The developed models simulate the sides of the VSS container with the explosive placed inside the container. Only cube (equal sides) containers were addressed in this study, so it was feasible to model only one quarter of the container to reduce the computational time. Different charges, container sizes and (effective) vent area ratios were taken into consideration in the developed models as will be discussed later in this chapter.

\subsection{Model Description (AUTODYN 2D model)}

Cubical VSS containers were modelled and investigated in the research program. The 2D models were developed to study the peak pressure and impulse attenuation outside the VSS containers as well as pressure on the side walls of the container. The centre of detonation of the charge was assumed to be in the center of the VSS container resulting in a spherical TNT blast wave propagating from the center of the container. Therefore the loading on and response of the suppressive shield elements on all sides of the container were the same. Three main parameters were investigated within the $2 \mathrm{D}$ modelling stage. The three parameters were the amount of TNT charge inside the container, the size of the container and the (effective) vent area ratio of the VSS container. Explosive charges varied between 1 to $20 \mathrm{~kg}$ TNT, while the container sizes modelled were $2.0 \times 2.0 \mathrm{~m}, 3.0 \times 3.0 \mathrm{~m}$ and $4.0 \times 4.0 \mathrm{~m}$. The studied vent area ratios of the VSS sections investigated were $0.16,0.25,0.50$ and 0.75 . The effect of the vent area ratio was studied in the case of the $2.0 \times 2.0 \mathrm{~m}$ container. Table 3 shows details for the 2 D AUTODYN models. 
Table 3: Properties of the developed 2D models

\begin{tabular}{|c|c|c|c|c|c|}
\hline $\begin{array}{c}\text { Explosive } \\
\text { Charge }(\mathrm{Kg})\end{array}$ & $\begin{array}{l}\text { Container } \\
\text { size }(\mathrm{m})\end{array}$ & Vent Area Ratio & $\begin{array}{c}\text { Explosive } \\
\text { Charge }(\mathrm{Kg})\end{array}$ & $\begin{array}{l}\text { Container } \\
\text { size }(\mathrm{m})\end{array}$ & Vent Area Ratio \\
\hline \multirow{8}{*}{1} & \multirow{4}{*}{$2.0 \times 2.0$} & 0.16 & \multirow{8}{*}{10} & \multirow{4}{*}{$2.0 \times 2.0$} & 0.16 \\
\hline & & 0.25 & & & 0.25 \\
\hline & & 0.50 & & & 0.50 \\
\hline & & 0.75 & & & 0.75 \\
\hline & \multirow{2}{*}{$3.0 \times 3.0$} & 0.25 & & \multirow{2}{*}{$3.0 \times 3.0$} & 0.25 \\
\hline & & 0.50 & & & 0.50 \\
\hline & \multirow{2}{*}{$4.0 \times 4.0$} & 0.25 & & \multirow{2}{*}{$4.0 \times 4.0$} & 0.25 \\
\hline & & 0.50 & & & 0.50 \\
\hline \multirow{8}{*}{5} & \multirow{4}{*}{$2.0 \times 2.0$} & 0.16 & & \multirow{4}{*}{$2.0 \times 2.0$} & 0.16 \\
\hline & & 0.25 & & & 0.25 \\
\hline & & 0.50 & & & 0.50 \\
\hline & & 0.75 & 20 & & 0.75 \\
\hline & \multirow{2}{*}{$3.0 \times 3.0$} & 0.25 & 20 & \multirow{2}{*}{$3.0 \times 3.0$} & 0.25 \\
\hline & & 0.50 & & & 0.50 \\
\hline & \multirow{2}{*}{$4.0 \times 4.0$} & 0.25 & & \multirow{2}{*}{$4.0 \times 4.0$} & 0.25 \\
\hline & & 0.50 & & & 0.50 \\
\hline
\end{tabular}

In transportation, it is not practical to use $3.0 \times 3.0$ or $4.0 \times 4.0$ containers because of the constraints of the width of the roads. Using this size of containers will require special permits and road closures during the transportation. However, these containers have been studied to investigate the effect of container size on the internal explosion as well as these containers can be used in the storage of hazardous materials but not for the transportation.

\subsubsection{Materials in 2D AUTODYN model}

The 2D AUTODYN model consisted of three components; the air domain for the medium for wave propagation, the TNT material that simulates the TNT charges for the explosive and the steel sections that form the side wall of the VSS container. The air and TNT were modeled with Euler mesh where the blast wave propagates through the cells without causing deformation to the mesh. The steel angles on the other hand were modeled using Lagrangean mesh where the cells are assumed to be deformable. Because at this stage of the 2D modelling, the point of interest was only tracking the pressure outside the container and on the side walls of the container, the failure of steel material was not defined. Table 4 presents the material properties and parameters for the Air, TNT and steel that were used in the 2D models. 
Table 4: Material properties of materials used in the model

\begin{tabular}{|c|c|}
\hline \multicolumn{1}{|c|}{ Air } \\
\hline Equation of State & Ideal Gas \\
\hline$\gamma$ & 1.4 \\
\hline Reference density $\left(\mathrm{g} / \mathrm{cm}^{3}\right)$ & $1.225 \mathrm{E}-03$ \\
\hline Reference Energy $(\mu \mathrm{J} / \mathrm{mg})$ & $2.068 \mathrm{E}+05$ \\
\hline Pressure Shift $(\mathrm{kPa})$ & 0 \\
\hline \multicolumn{1}{|c|}{$\mathrm{TNT}$} & $\mathrm{JWL}$ \\
\hline Equation of State & 1.35 \\
\hline$\gamma \quad 1.00 \mathrm{E}-04$ \\
\hline Reference density $\left(\mathrm{g} / \mathrm{cm}^{3}\right)$ & $2.07 \mathrm{E}+05$ \\
\hline Reference Energy $\left(\mu \mathrm{J} / \mathrm{mg}^{3}\right)$ & 0 \\
\hline Pressure Shift $(\mathrm{kPa})$ &
\end{tabular}

The air sub grid used the Euler-FCT processor and was filled with a material model, which used the ideal gas equation of state (Table 4) to simulate the domain of the model where the blast wave propagates. TNT charge was modeled in AUTODYN using JWL equation of state model as shown in Table 4. When the TNT charge is detonated, the TNT material decomposes and then expands (Century Dynamics Inc., 2005). The JWL equation of state model is a valid representation of the TNT up to a TNT volume of 10 times its original volume. Beyond this volume, the TNT material properties change to ideal gas and thus the ideal gas equation of state model is considered to be accurate in simulating the TNT material. This modification should be taken into consideration in the model to avoid inaccurate pressure values in the AUTODYN model. More details about this point will be addressed in the remapping section.

\subsubsection{Boundary conditions of 2D AUTODYN model}

In 2D modelling, the container was modelled as a square box. Only one quarter of the box shape was modelled to reduce the computation time. Symmetric boundary conditions were defined at the axis of symmetry of the AUTODYN model. Defining symmetry boundary condition corresponds to the physical assumption that, on the two sides of symmetry axis, the same physical processes exist. The variable values at the same distance from the boundary at the two sides are the same. Also, the function of such a boundary is that it acts as a mirror that reflects all the fluctuations generated by the simulation region. Figure 22 shows the axis of symmetry where the symmetry boundary conditions were assigned. 


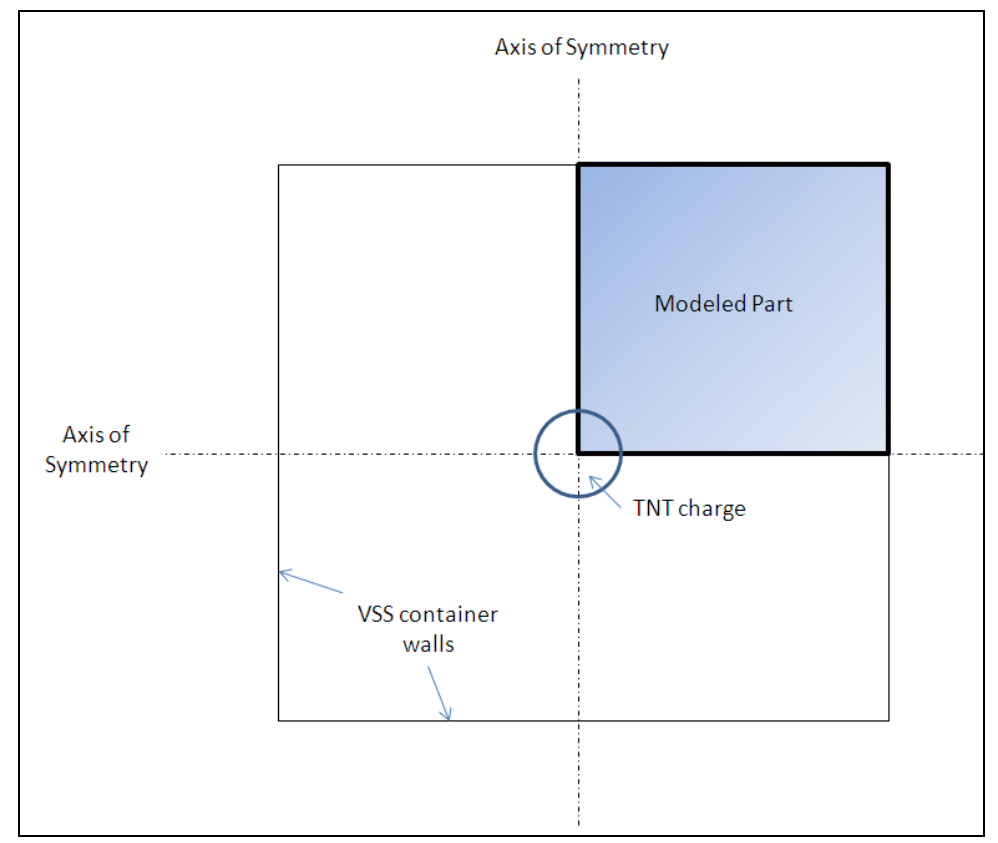

Figure 22: Plan view of VSS container

Two other types of boundary conditions were also defined in the AUTODYN models. The first type was known as outflow boundary condition. This boundary condition was assigned to the outer perimeter of the air domain as shown in Figure 23. Outflow boundary conditions are used to model flow exits where the details of the flow velocity and pressure are not known prior to solution of the flow problem. The purpose of having an outflow boundary condition along the outer perimeter of the model was to allow the blast pressure to escape outside the model and prevent it from reflecting back to the model that may affect the results.

The third boundary condition that was defined in the 2D AUTODYN models was the fixed boundary condition known as "Zero velocity" boundary condition. Fixed boundary conditions can only be applied on Lagrange elements in order to prevent their movement or deformation due to the applied load. The movement and deformation of steel angles were prevented in order to have a good understanding of the propagation of blast waves around the steel plates. This is because if the angles were set free to deform or move, the effective vent area ratio of the VSS section will be affected and thus the performance of the VSS section will be affected too. Figure 23 shows the assigned boundary conditions as well as the axis of symmetry mentioned earlier. 


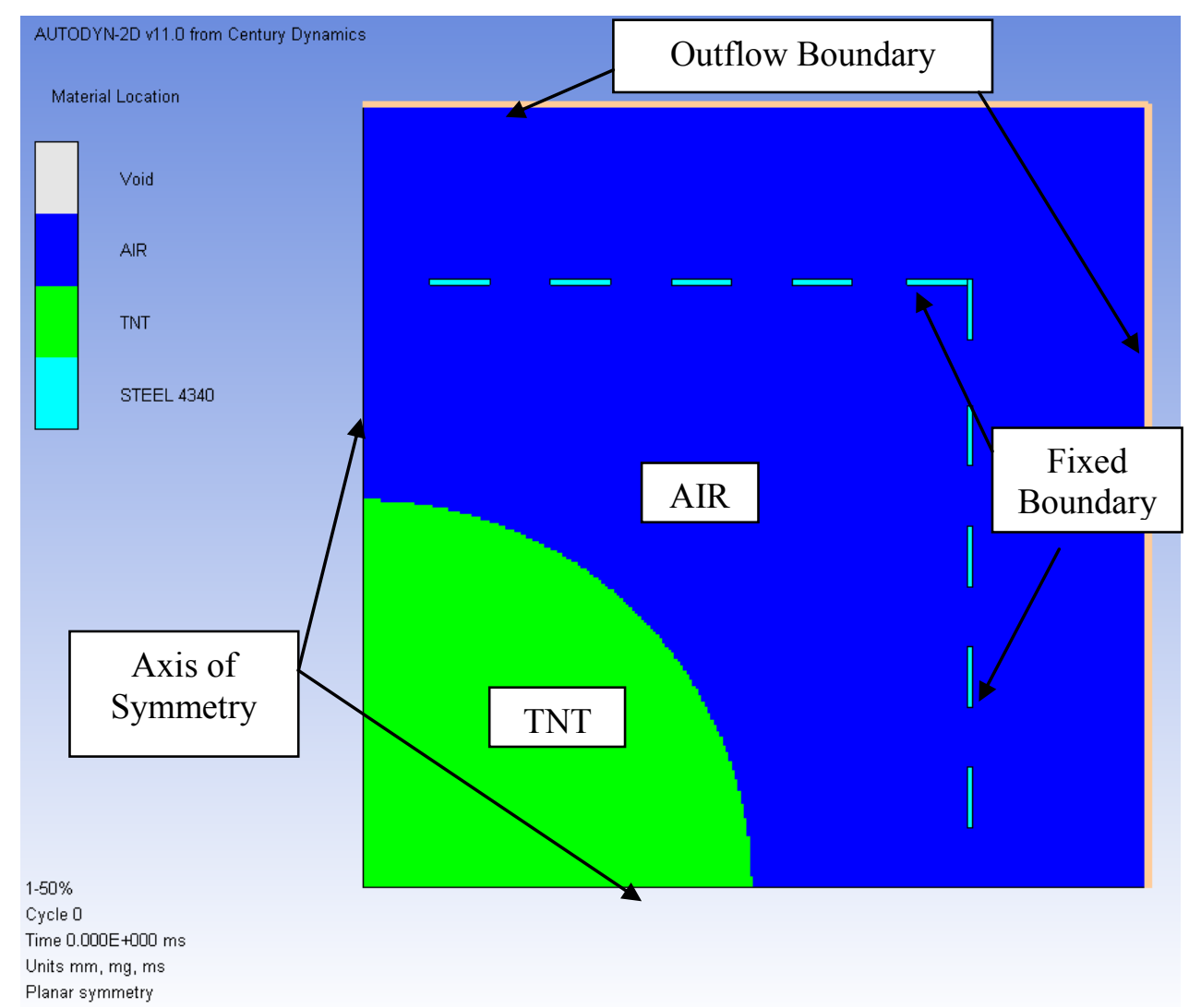

Figure 23: Axis of symmetry and boundary conditions

\subsubsection{Gauges in 2D AUTODYN model}

Tracking the pressure value outside the container and on the steel angle was the main objective in the 2D modelling. Gauges were used to track the pressure at various locations outside the container and on the side walls of the VSS container. Gauges outside the container (G1 to G10) were located at distances from 2 to $10 \mathrm{~m}$ spaced at $2 \mathrm{~m}$ spacing (Figure 24). The rest of the Gauges were located just in front of the inside face of the steel angles in order to track the reflected pressure at the steel angles. Tracking these pressure values outside the container reflects the performance of the steel section in attenuating peak blast pressure. 


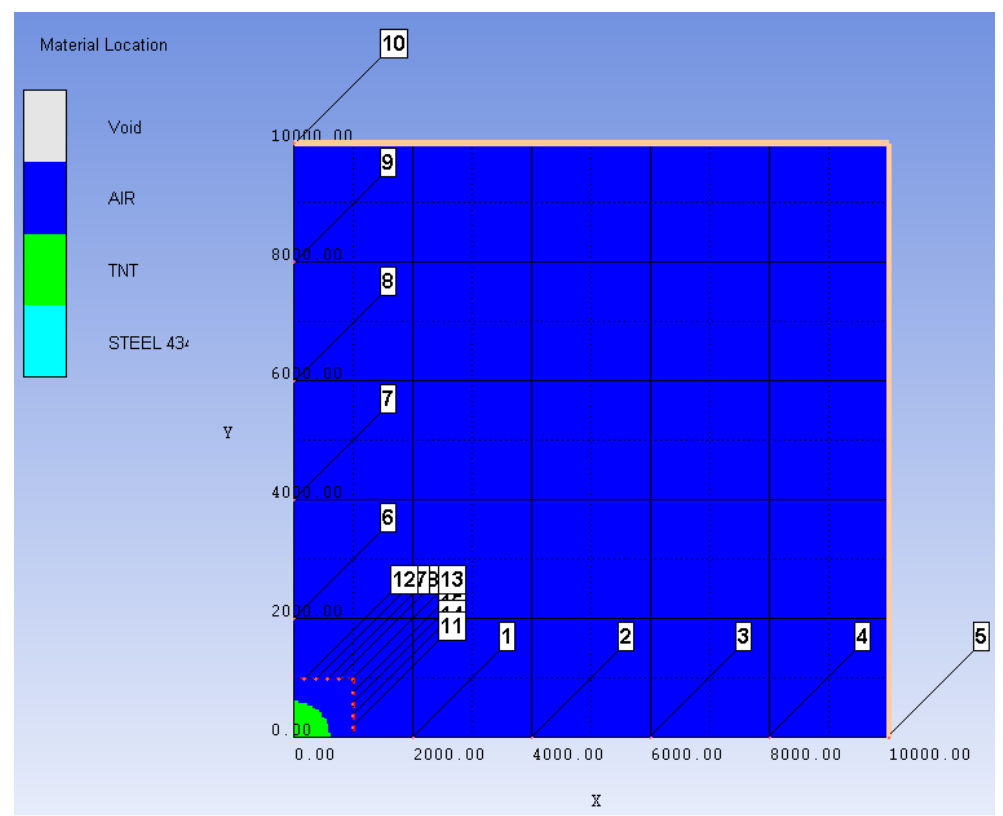

Figure 24: Location of Gauges outside the container and on the VSS elements

\subsubsection{Remapping}

To model the TNT charge in the air domain, a part of the air domain has to be filled with TNT material that is equivalent to the amount of explosives being modelled. The radius of the TNT charge was calculated using Equation 22. In order to reduce the computation time of the AUTODYN model, a remapping technique was used to simulate the TNT charge. Remapping is a technique used in AUTODYN where the TNT charge is imported from another file that consists of an air wedge filled with TNT material to simulate the TNT charge. The model representing the wedge was run and stopped just before the point of interest. The file was then saved and imported to the original AUTODYN file that includes the steel structure. In the remapping file, the radius of the TNT charge was also calculated by Equation 22. For hemispherical charges, the radius of the TNT charges in Equation 22 is multiplied by 1.2 .

$$
R=100 \times \sqrt[3]{\frac{3 \times W}{\rho_{T N T} \times 4 \times \pi}}
$$

Equation 22

Where, $R$ is the radius of spherical charge $(\mathrm{mm}), W$ is mass of charge $(\mathrm{kg})$ and $\rho_{T N T}$ is density of TNT $=1.63 \mathrm{~kg} / \mathrm{m}^{3}$ 
As mentioned earlier in this chapter, the material properties of the TNT changes when its volume exceeds 10 times the original volume. At this point the material properties of TNT are converted to ideal gas (Century Dynamics Inc., 2005). Figure 25 represents an example of remapping file for a $10 \mathrm{~kg}$ TNT charge expansion to a time $0.285 \mathrm{msec}$. At this time, the volume of the TNT charge is 10 times its original volume. The model was stopped at this point and new material properties were defined and then the model was run again until it reaches the point of interest. In the case of the smallest container $(2.0 \times 2.0 \mathrm{~m})$ the remap file contains a TNT charge expansion to $0.8 \mathrm{~m}$ away from the center of charge. Figure 25 shows contour lines showing the pressure values within the air wedge.

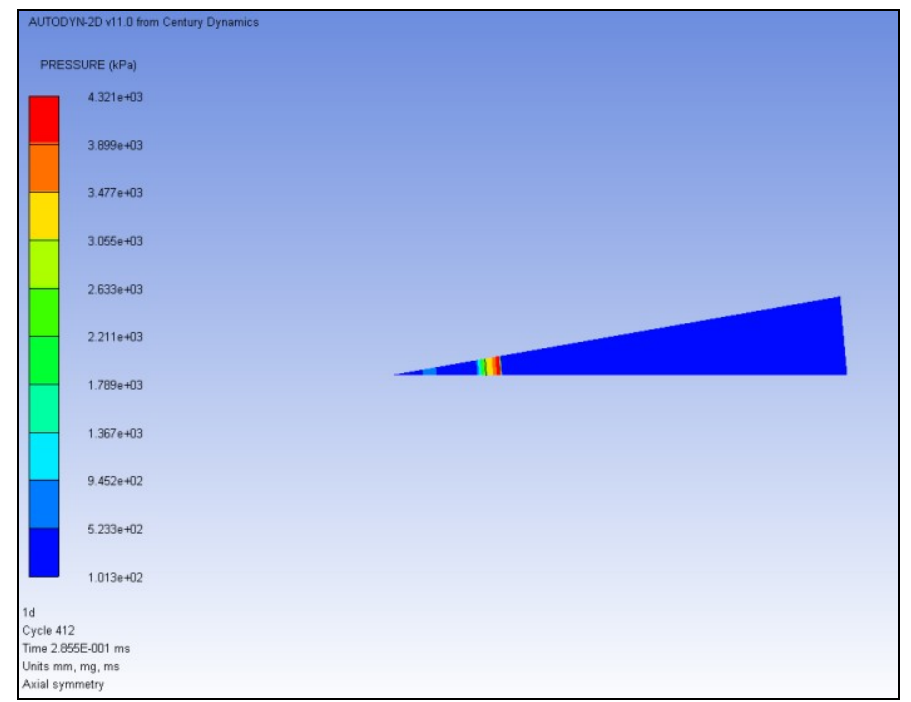

Figure 25: Remapping file for $10 \mathrm{~kg}$ TNT

\subsection{AUTODYN 3D modelling}

Development of 3D AUTODYN models was to study the behaviour of the VSS elements under the effects of blast loading. From the 2D modelling, it was found that the performance of VSS sections with vent area ratio of 0.16 and 0.75 was very poor when compared to other vent area ratios so these two vent area ratios were not modelled in the $3 \mathrm{D}$ modelling portion. This will be discussed in details in chapter 5. Table 5 shows the models that were developed in $3 \mathrm{D}$ AUTODYN. 
Table 5: Properties of the developed 3D models

\begin{tabular}{|c|c|c|c|c|c|}
\hline $\begin{array}{c}\text { Explosive } \\
\text { Charge (kg) }\end{array}$ & $\begin{array}{c}\text { Container } \\
\text { size }(\mathrm{m})\end{array}$ & Vent Area Ratio & $\begin{array}{c}\text { Explosive } \\
\text { Charge (kg) }\end{array}$ & $\begin{array}{l}\text { Container size } \\
\text { (m) }\end{array}$ & Vent Area Ratio \\
\hline \multirow{6}{*}{1} & \multirow{2}{*}{$2.0 \times 2.0$} & 0.25 & \multirow{6}{*}{10} & \multirow{2}{*}{$2.0 \times 2.0$} & 0.25 \\
\hline & & 0.5 & & & 0.5 \\
\hline & \multirow{2}{*}{$3.0 \times 3.0$} & 0.25 & & \multirow{2}{*}{$3.0 \times 3.0$} & 0.25 \\
\hline & & 0.5 & & & 0.5 \\
\hline & \multirow{2}{*}{$4.0 \times 4.0$} & 0.25 & & \multirow{2}{*}{$4.0 \times 4.0$} & 0.25 \\
\hline & & 0.5 & & & 0.5 \\
\hline \multirow{6}{*}{5} & \multirow{2}{*}{$2.0 \times 2.0$} & 0.25 & \multirow{6}{*}{20} & \multirow{2}{*}{$2.0 \times 2.0$} & 0.25 \\
\hline & & 0.5 & & & 0.5 \\
\hline & \multirow{2}{*}{$3.0 \times 3.0$} & 0.25 & & \multirow{2}{*}{$3.0 \times 3.0$} & 0.25 \\
\hline & & 0.5 & & & 0.5 \\
\hline & \multirow{2}{*}{$4.0 \times 4.0$} & 0.25 & & \multirow{2}{*}{$4.0 \times 4.0$} & 0.25 \\
\hline & & 0.5 & & & 0.5 \\
\hline
\end{tabular}

In the $3 \mathrm{D}$ modelling of the VSS container, the container consisted of a main frame and the side walls of the container. The main frame was not modelled in case of the $2 \mathrm{D}$ models because it was outside the point of interest at that stage but it was modelled in the 3D model because it will act as the support to the steel members of the side wall of the VSS container. The main frame was assumed to be a hollow box section as shown in Figure 26. The side walls as well as the main frame of the VSS container were modelled with Lagrangean shell elements. The steel angles (plates) forming the VSS side wall were connected to the main frame of the container by rigid connection to simulate the welded connection between the steel angles forming the sides of the container and the main frame of the container. The steel elements of container's side walls were allowed to deform. In contrast to the $2 \mathrm{D}$ model where the VSS elements were fixed in order study the propagation of the blast wave. Figure 26 shows an example of a 3D model for one of the containers. 


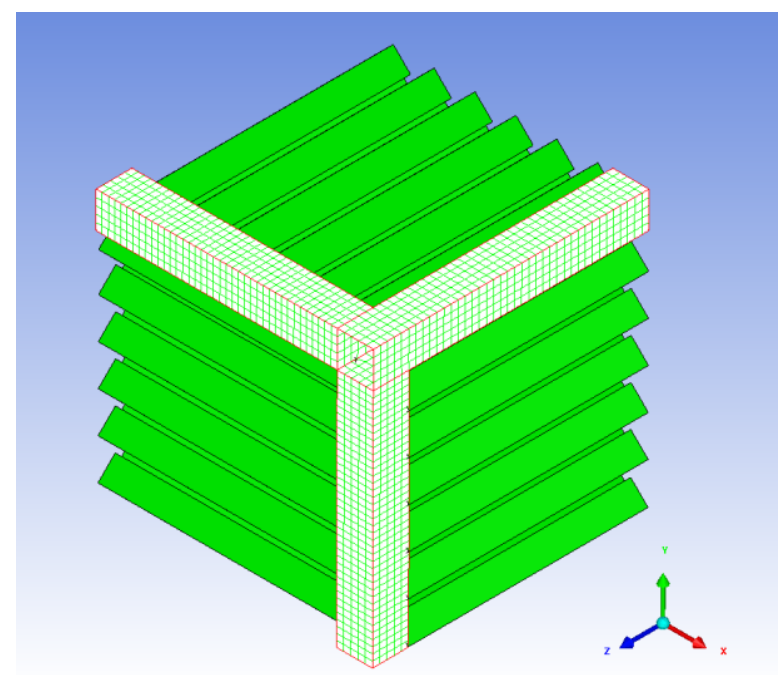

Figure 26: Example for 3D VSS container model

\subsubsection{Materials in 3D AUTODYN model}

The material models used in the 3D model were the same as those used in the 2D model. However in the 3D model, the failure criterion for the steel was defined as shown in Table 6 . The Johnson-Cook model was used to define the material strength of the steel. The material failure was defined at a plastic strain of 0.02 as well the erosion of the material was assigned to be at a geometric strain of 0.02 .

Table 6: Material Properties in 3D model

\begin{tabular}{|c|c|}
\hline \multicolumn{2}{|c|}{ Steel } \\
\hline Equation of State & Linear \\
\hline Reference density $(\mathrm{g} / \mathrm{cm} 3)$ & 7.83 \\
\hline Bulk modulus $(\mathrm{kPa})$ & $2.00 \mathrm{E}+08$ \\
\hline Reference temperature $(\mathrm{K})$ & 300 \\
\hline Specific heat capacity $(\mathrm{J} / \mathrm{kgK})$ & 477 \\
\hline Shear modulus $(\mathrm{kPa})$ & $8.18 \mathrm{E}+07$ \\
\hline Yield Stress $(\mathrm{kPa})$ & $4.00 \mathrm{E}+05$ \\
\hline Hardening constant $(\mathrm{kPa})$ & $5.10 \mathrm{E}+05$ \\
\hline Hardening exponent & 0.26 \\
\hline Strain rate constant & 0.002 \\
\hline Thermal softening exponent & 1.03 \\
\hline Melting temperature $(\mathrm{K})$ & 1793 \\
\hline Failure model Plastic strain & 0.02 \\
\hline Erosion model Geometric strain & 0.02 \\
\hline
\end{tabular}


It should be noted that the erosion mentioned in the table is not a physical phenomenon. Erosion strain is a numerical technique used in AUTODYN software to overcome problems that may occur in the model due to the distortion of Lagrange sub grids. When a Lagrange sub grid experiences large deformation or distortion due to the applied load, the sub grid loses its strength. However, the mass of these sub grids is still taken into account in the computations. So erosion strain was defined in order to discard the sub grids that experienced distortion due to the large deformations. If the eroded sub grids are needed for other computations, a retained inertia option can be chosen and thus the inertia of these sub grids can be used again in the computations (Century Dynamics Inc., 2005).

\subsubsection{Boundary conditions in 3D AUTODYN model}

In the 3D modelling, only one-eighth of the container was modelled, so symmetry boundary conditions were also used in the $3 \mathrm{D}$ model as in case of $2 \mathrm{D}$ models. In case of $2 \mathrm{D}$ modelling, the symmetry boundary condition is applied on the line of symmetry while for in the $3 \mathrm{D}$ modelling, the symmetry boundary condition is applied on the planes of symmetry. Figure 27 shows an example to where the symmetry boundary condition could be applied in a 3D problem.

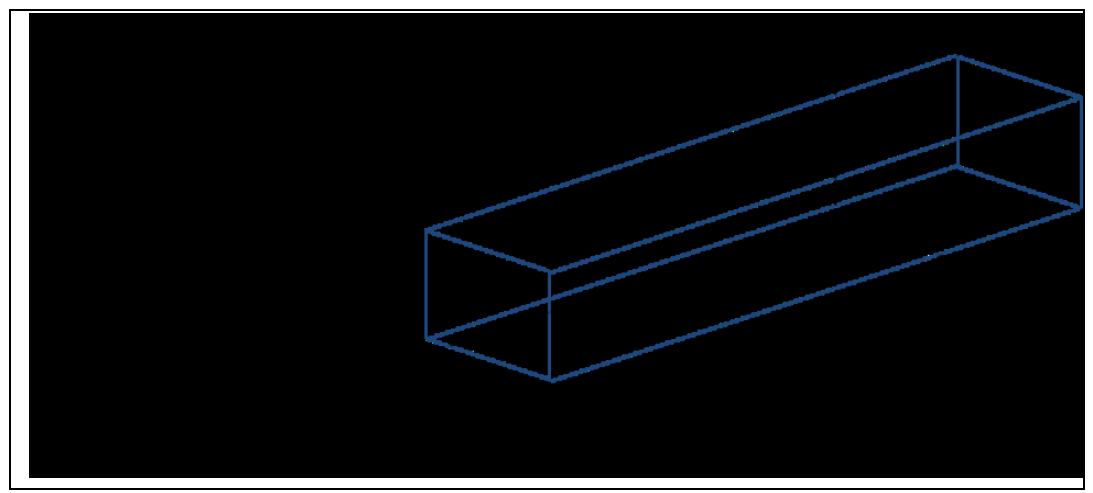

Figure 27: Symmetry boundary conditions

The main frame was modelled in the $3 \mathrm{D}$ model to simulate the edges of the container. The main frame was assumed to be the support for the steel VSS elements, so a higher steel grade with $400 \mathrm{MPa}$ yield strength was used to simulate the main frame to ensure that the main frame was stronger than the steel VSS elements. The connection between the steel VSS elements and the frame was assumed to be a welded connection. For the modelling, the steel VSS elements were assumed to be rigidly connected to the frame. The main frame is simulated as 
hollow section as shown in Figure 28. In order to study the behaviour of the steel VSS elements, the main frame was assumed to be fixed to prevent any deformation or movement to the main frame that may affect the behaviour of the steel VSS elements forming the side wall of the VSS container.

As in case of the 2D model, outflow boundary condition were also applied at the outside planes of the air domain to allow the velocity vectors to escape outside the model after passing through the container. Figure 28 shows the fixed boundary conditions at the main frame of the container as well as the velocity vectors from the remapped file simulating the TNT charge.

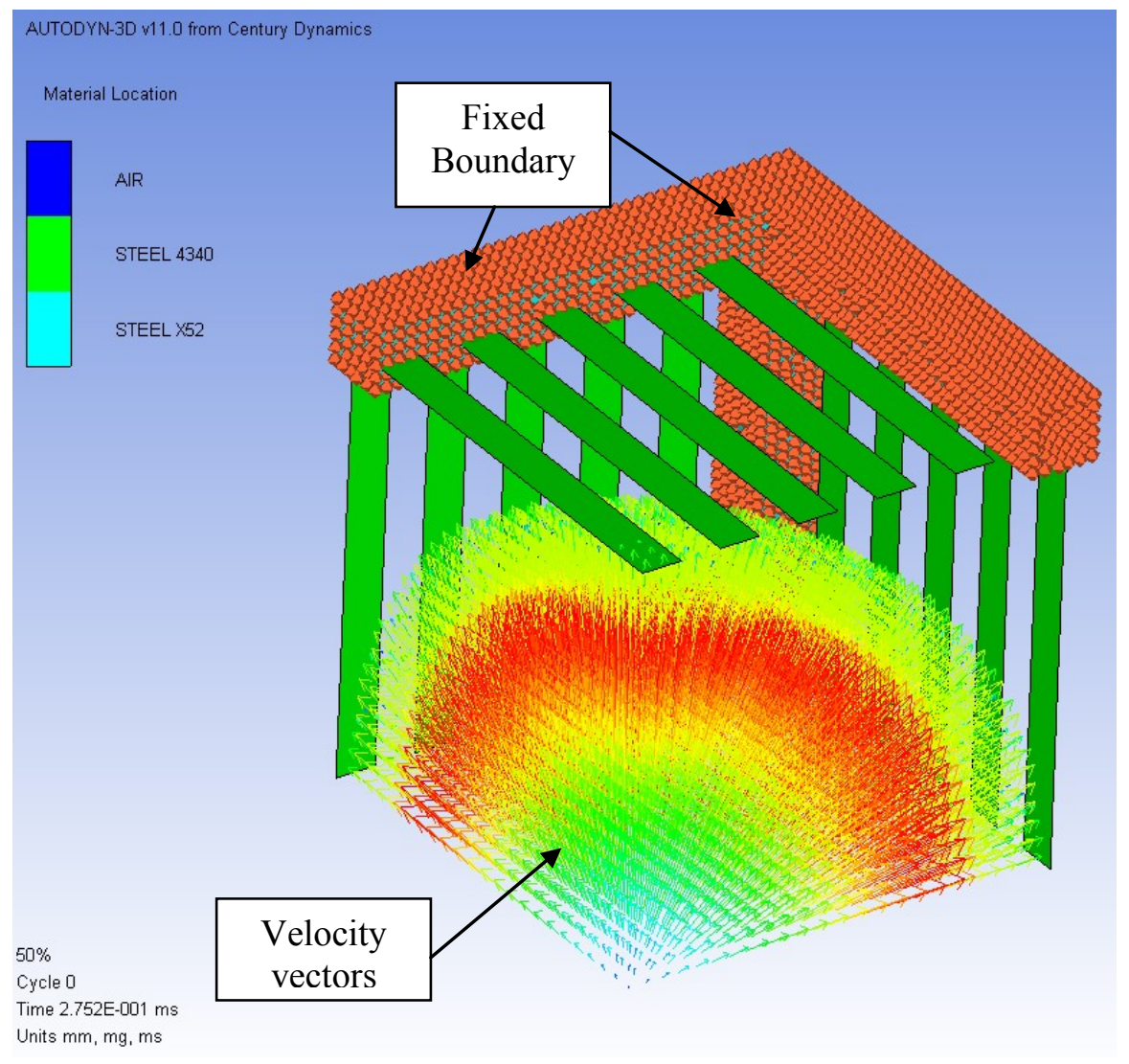

Figure 28: Fixed boundary condition at main frame

\subsubsection{Gauges in 3D AUTODYN model}

Two types of Gauges were used in the 3D models to record pressure and impulse history during the simulation: fixed and movable. The fixed Gauges were used to record pressure on the VSS elements as well as the pressure outside the container at various distances. The fixed 
Gauges are assigned to the Euler mesh where they remain fixed with the mesh throughout the simulation. The fixed Gauges were used to record the pressure on the VSS elements and were placed just in front of the inside face of the VSS elements.

The movable Gauges were used to monitor deformations of the structural elements. These Gauges were fixed to specified locations on the VSS elements and in addition to deformations also, recorded the velocity and accelerations of the VSS elements. Figure 29 shows a 3D model of a suppressive shield container and Gauge locations, at the mid-span of the steel plates.

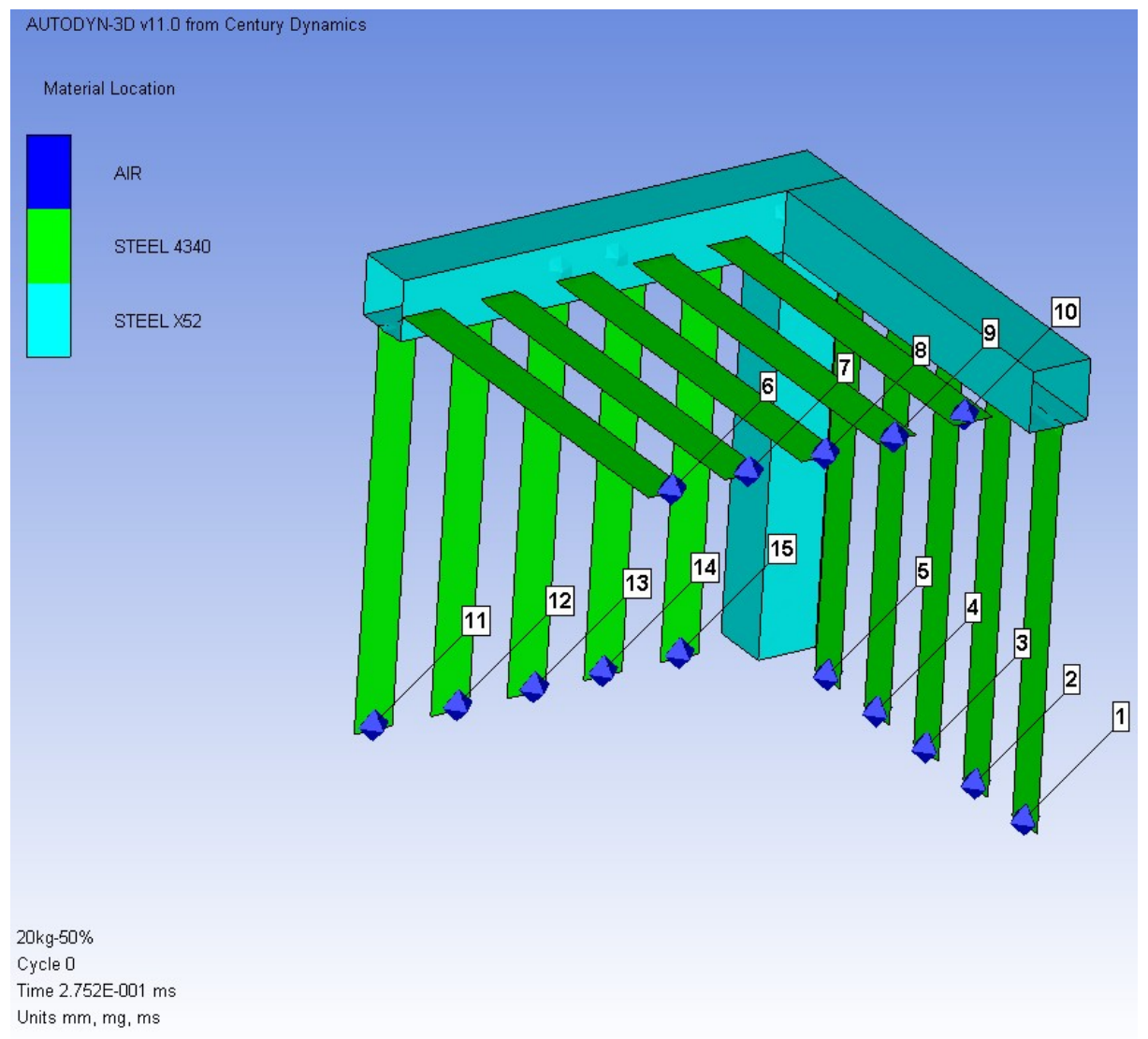

Figure 29: Gauges on steel plates

\subsubsection{Shell sub grid elements}

The steel angles or plates forming the VSS side walls as well as the main frame of the VSS container were modelled as shell elements. Shell elements are often assigned to twodimensional parts such as membranes. Using shell elements enables efficient computation in 
spite of their small thickness of the membrane. Since all the sections used are steel plates and hollow sections, shell elements were deemed appropriate rather than regular plate elements. In finite elements, shell elements are different from plate elements in the following:

- They can be curved,

- They carry membrane and bending forces, and

- A thin shell structure can carry high loads if membrane stresses predominate.

However, localized bending stresses may appear near load concentrations or geometric discontinuities in case of shell elements. In AUTODYN software, the shell element can be solved in one of the two solution solvers:

- Bending ( 2 or 3 sub layers): This technique considers the element only behaving in bending. The direction of bending should also be defined in the model. This technique is mostly used for regular thick section where the direction of bending is clear and can be easily defined.

- Membrane: It is recommended to use the membrane solver when modelling very thin members. In this solver, it is possible to track the stresses and strains at the four vertices of the steel plate and by interpolation, the stresses in the whole section could be calculated.

As a result, the membrane solver technique was used to model the shell sub grid in the developed 3D AUTODYN models. The shell sub grid used the Lagrange processor and was filled with a material model, which used the linear equation of state, the Johnson Cook strength model, the bulk strain failure model and the erosion model.

\subsection{Welded connections}

The connections between the steel elements forming the side walls of the VSS and the main frame of the container was assumed to be weld connection. The nodes of the VSS elements were assumed to be rigidly connected to the main frame of the container. Figure 30 shows the connection between the steel plates of the VSS side wall and the main frame of the container. 


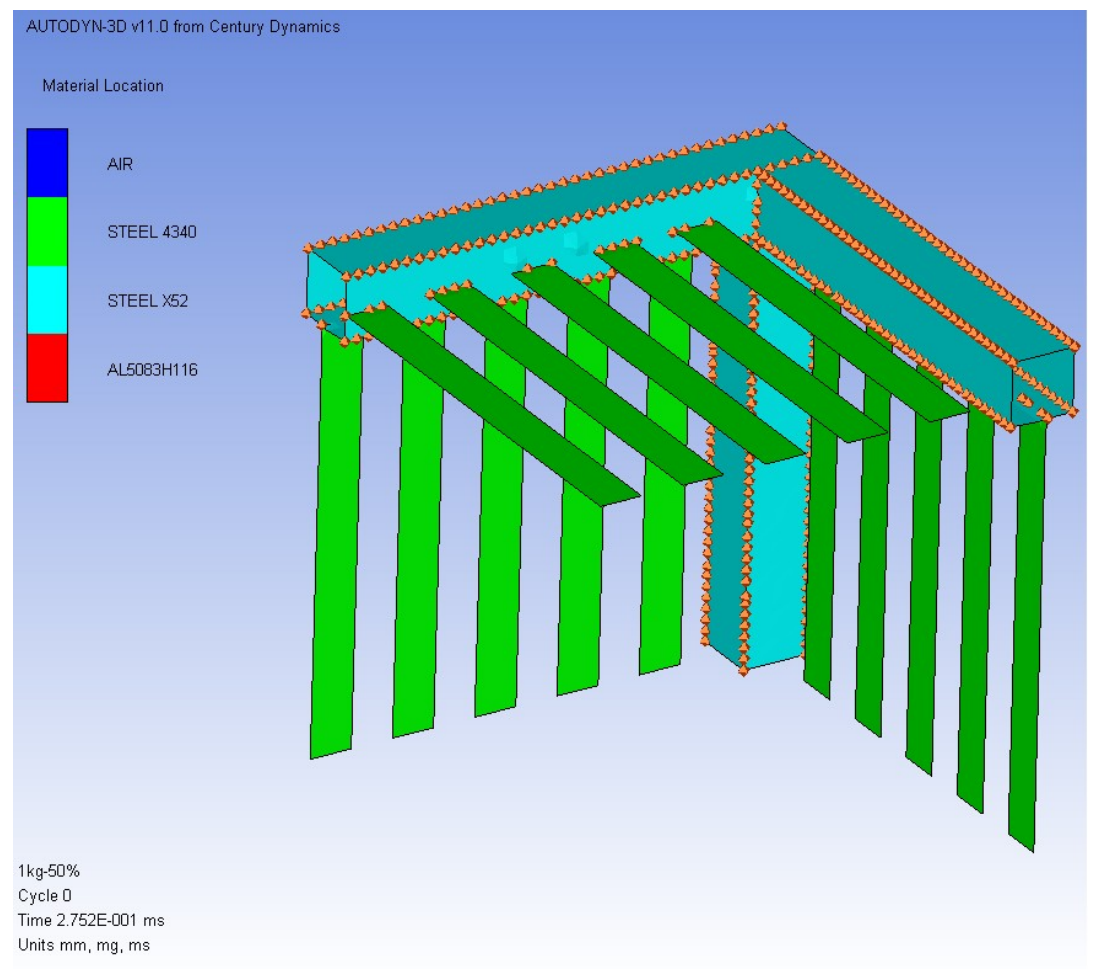

Figure 30: Connection between steel plates and the main frame

\subsection{Summary}

This chapter presented a detailed explanation for the modelling part using AUTODYN. The following two chapters; chapter 4 and 5 will present and discuss the results obtained from these developed models as well as verification for the developed models. 


\section{Chapter 4: $\quad$ Pressure and Impulse attenuation outside VSS containers}

\subsection{Introduction}

This chapter addresses the computational methods to calculate the effective vent area ratio of the studied VSS sections. This is done for both single and multilayer VSS sections. The chapter also discusses the level of attenuation of the different VSS sections and the comparison between them. From the results obtained from the AUTODYN models, a set of empirical equations are developed to calculate the pressure and impulse values outside vented structures. The proposed equations are then validated by comparing the output of those equations with previous experimental data by other researchers.

\subsection{Vent area ratio for VSS geometric configurations}

Vent area ratio $(\alpha)$ defines the amount of vents present in a VSS section. Calculating the vent area ratio for VSS sections has posed challenges to designers and researchers as the VSS configurations can vary widely. There is no general equation to calculate the vent ratio for different VSS sections. The equation to calculate the vent ratio differs from one geometric configuration to another. In the following section, the vent area ratio for different VSS sections will be studied and new equations are proposed to calculate the vent area ratio for specific VSS sections. Five single layer VSS sections and two multi-layer VSS sections were investigated in the research program.

\subsubsection{Single-Layer VSS configurations}

Single layer VSS is a vented suppressive shield section that is formed from one layer of VSS elements. These elements can be made of plates or angles depending on the geometric shape. The gaps between the elements define the vent ratio of the VSS section. The following sections present a detailed study of different single layer VSS sections and their effect on the level of blast pressure and impulse attenuation outside the VSS container.

\subsubsection{Single-Layer Plate Configuration}

The single-layer plate configuration is the simplest single layer VSS configuration for studying the effects of vent area ratio on the blast pressure and impulse attenuation capacity. The plate and gap sizes between them were varied to yield different vent area ratios while the 
thickness of the plates was kept constant. Figure 31 shows a single-layer plate section. The length of each plate is denoted as $l$, the gap between plates as $a$, while the overall length of the single-layer plate VSS is denoted as $M$. The number of plates is denoted as $n$.

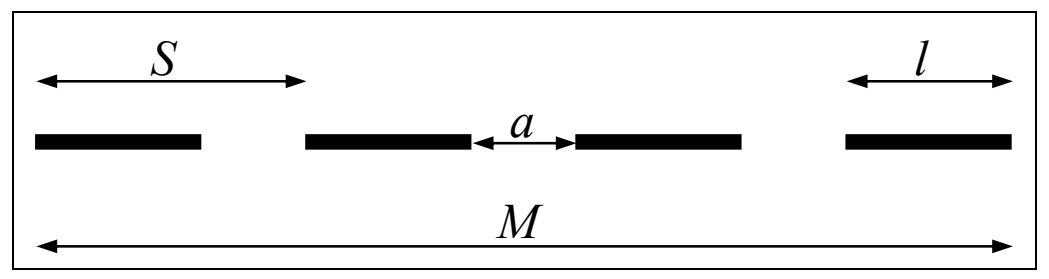

Figure 31: Single layer plate configuration

The vent area ratio $(\alpha)$ for the single-layer plate configuration is defined as the ratio of vent area $\left(A_{\text {vent }}\right)$ to the total area $\left(A_{\text {total }}\right)$ of the VSS section as expressed in Equation 23.

$$
\alpha=\frac{A_{\text {vent }}}{A_{\text {total }}}=\frac{(n-1) a}{M}=\frac{M-n l}{M}=\left(1-\frac{l}{M}\right)
$$

Equation 23

\subsubsection{Single-layer inclined plate configuration}

One of the main disadvantages of using the single-layer plate configuration is the presence of direct paths for blast wave propagation to the exterior and exit of primary fragments. Eliminating fragments is one of the main goals of the VSS container design. The single-layer inclined plate configuration uses inclined plates to prevent direct blast wave propagation and primary fragment exit to the exterior. Figure 32 presents the single-layer inclined plate configuration.

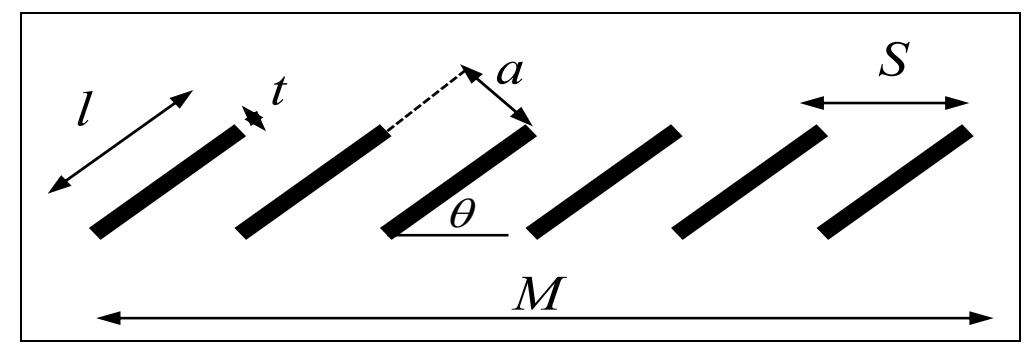

Figure 32: Single-layer inclined plate 
The vent area ratio depends on the plate inclination $(\theta)$, plate spacing $(S)$, thickness $(t)$ of the plates and the length of the plates $(l)$. The number of inclined plates in the VSS section is denoted as $n$. The vent area ratio $(\alpha)$ of the single-layer inclined plate configuration is given by Equation 24.

$$
\alpha=\frac{(n-1) a}{M}=\frac{(n-1)(S \sin \theta-t)}{M}
$$

Equation 24

Where $l \cos \theta \geq s-t \sin \theta$ the direct path for fragment exit to the exterior is eliminated. Vent area of the single-layer inclined plate configuration is defined based on the minimum area available for blast wave propagation - the perpendicular distance between incline plates; $a=$ $s \times \sin \theta-t$. The values of $\theta$ and $s$ are chosen to eliminate a direct path for blast wave propagation and primary fragment exit from the VSS container. For a given single-layer inclined plate configuration, increasing the plate angle of inclination increases the vent area ratio, until $\theta=90^{\circ}$ when the vent area ratio approaches that of the single-layer plate configuration with plate length equal to the plate thickness, $t$.

\subsubsection{Single-layer side-by-side angle configuration}

The single-layer side-by-side angle configuration consists of equal-leg steel angles spaced at $\mathrm{s}$. The single-layer side-by-side angle configuration can be either exterior-facing (Figure 33(a)) or interior-facing (Figure 33(b)). The single-layer side-by-side angle configuration was introduced by the U.S. Army (USACE, 1977). The single-layer side-by-side angle configuration is similar to the single-layer plate configuration but with the advantage of oblique blast wave reflection off the steel angles. The oblique reflection leads to a reduced reflected pressure on the steel angles, especially on the exterior-facing configuration. 

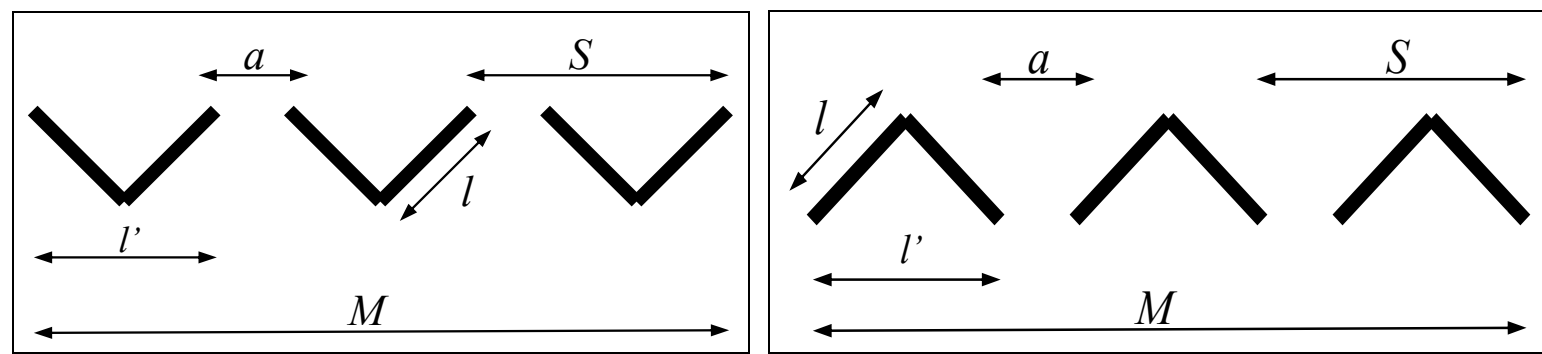

Figure 33: (a) Single-layer side-by-side angle exterior-facing, (b) Single-layer side-by-side angle interiorfacing

The vent area ratio of the single-layer side-by-side angle configuration is given by Equation 25.

$$
\alpha=\frac{(n-1) a}{M}=\frac{M-2 n l \cos 45^{\circ}}{M}=1-\frac{2 n l \cos 45^{\circ}}{M}
$$

Equation 25

Where $l$ is the length of the leg of the equal-leg angle, $n$ is the number of steel angles, and $L$ the overall length of the VSS. The angle of inclination of the legs of the angle to the horizontal is $45^{\circ}$.

\subsubsection{Single-layer nested angle configuration}

The single-layer nested angle configuration has similar properties as the single-layer inclined plate configuration (Figure 34). As with the single-layer side-by-side angle configuration, the single-layer nested angle configuration was introduced in the 1970 (USACE, 1977).

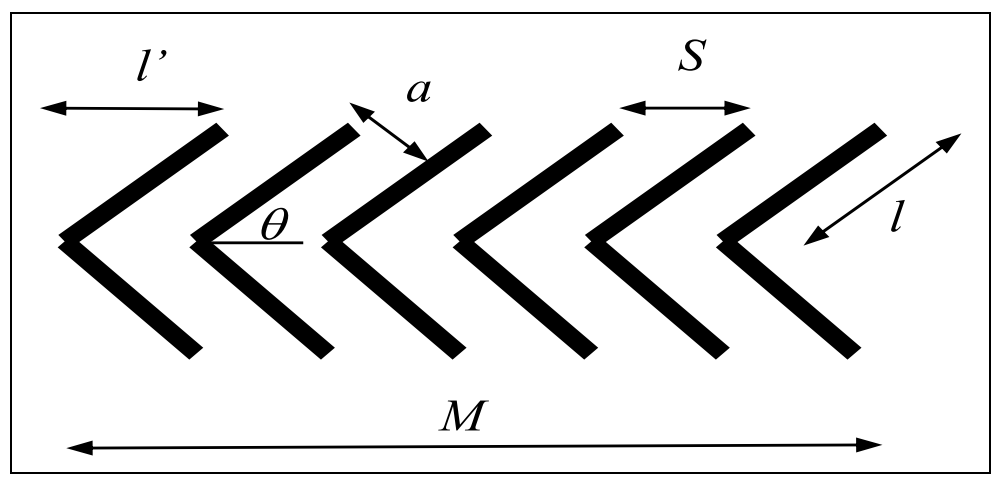

Figure 34: Nested angles 
The calculation procedure of the vent area ratio of single-layer nested angle configuration is same as that of the single-layer inclined plate configuration. The difference between the single-layer nested angle configuration and the single-layer inclined plate configuration is that the equal leg steel angles are inclined at $45^{\circ}$. The vent area ratio of the single-layer nested angle is calculated using the same equation used for the inclined plate configuration (Equation 24). In order to ensure that this section will eliminate the fragments exit to the exterior, the gap $(a)$ should not exceed the projected length of the steel angle.

\subsubsection{Multi-Layer VSS configurations}

The addition of a second layer of steel elements to the single-layer plate configuration eliminates the direct path for blast pressure wave propagation and primary fragment exit to the exterior. The multi-layer VSS configurations consist of a combination of one or more singlelayer section arranged in a staggered configuration.

\subsubsection{Double-Layer Plate Configuration}

The double-layer plate configuration consists of two single-layer plate configurations arranged in a staggered fashion to eliminate direct paths to the exterior of the VSS container. The vent area ratio for the multilayer VSS configurations is known by effective vent area ratio and is calculated using Equation 2 mentioned earlier. In addition to the properties of single-layer plate configurations the gap distance between the two layers, $g$, will be taken into account in this study. Figure 35 presents the pertinent dimensions of the double-layer plate configurations.

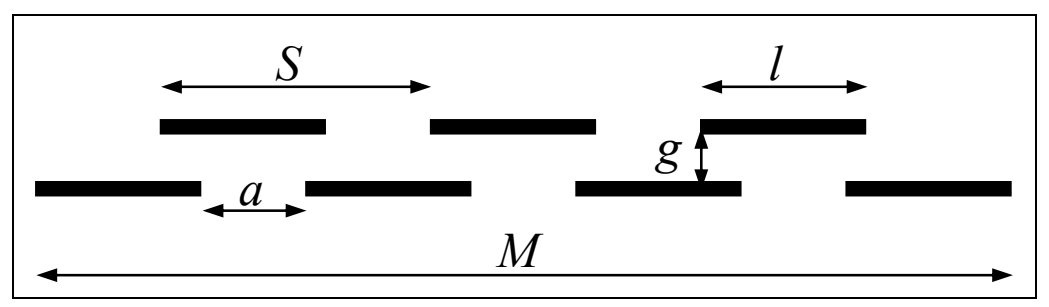

Figure 35: Double-layer plate configuration

\subsubsection{Double-Layer Nested Side-By-Side Angle Configuration}

The double-layer nested side-by-side angle configuration shown in Figure 36 is also known as Category 5 VSS configuration. The configuration was introduced by Koger et al., 
(1975). It was designed to contain accidental detonation of explosives, and to reduce the flame and fireball from explosion reaction to a tolerable level.

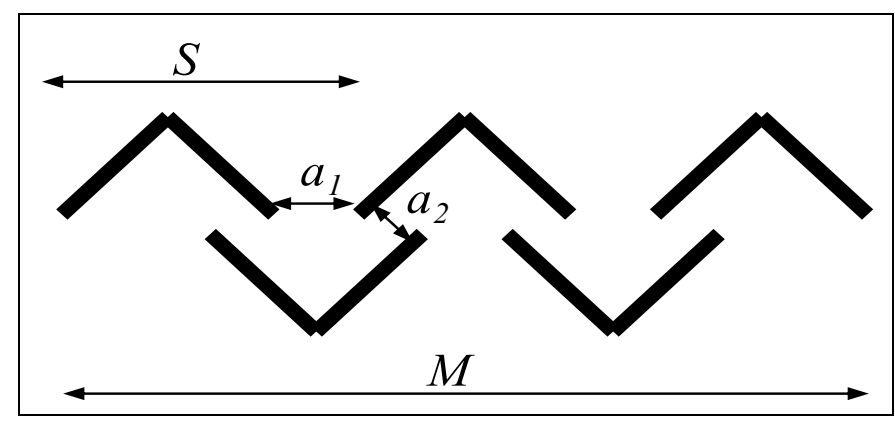

Figure 36: Double-layer nested side-by-side angle configuration (Koger et al., 1975)

\subsection{Sensitivity Analysis of AUTODYN model}

Mesh size in finite element modeling plays a significant role in the accuracy of the results. As the mesh size is refined, the more accurate are the results. However, the computation time is much longer. A sensitivity analysis was conducted to establish a mesh size that minimized computational effort while maximising modelling accuracy. The accuracy of the results was determined by comparing the results obtained from AUTODYN models with the results obtained from Conventional Weapons Effects Program (ConWep) computer program. ConWep is a computer program that has been used for determining free-field pressures and loads on structures. ConWep software is based on set of equations developed by Kingery and Bulmash (1984). These equations are used to predict air blast parameters for both spherical and hemispherical surface bursts. These equations are widely accepted as engineering predictions and ConWep has been known as a good tool for predicting blast loads on structures (Swisdak Jr. 1994).

An AUTODYN model was developed simulating an air domain with a $20 \mathrm{~kg}$ TNT spherical charge and the pressure was recorded at different distances from center of detonation (standoff distance). Gauges were located at different distances from the charge: 1, 2, 4, 6, 8 and $10 \mathrm{~m}$. This model was modelled several times with different mesh sizes and the pressure was recorded for each case and compared to the pressure values obtained from ConWep. The mesh sizes that were used are 1, 5, 10 and $20 \mathrm{~mm}$. Figure 37 shows the developed 2D AUTODYN model that was used to study the sensitivity of mesh sizes on the accuracy of the pressure values. 


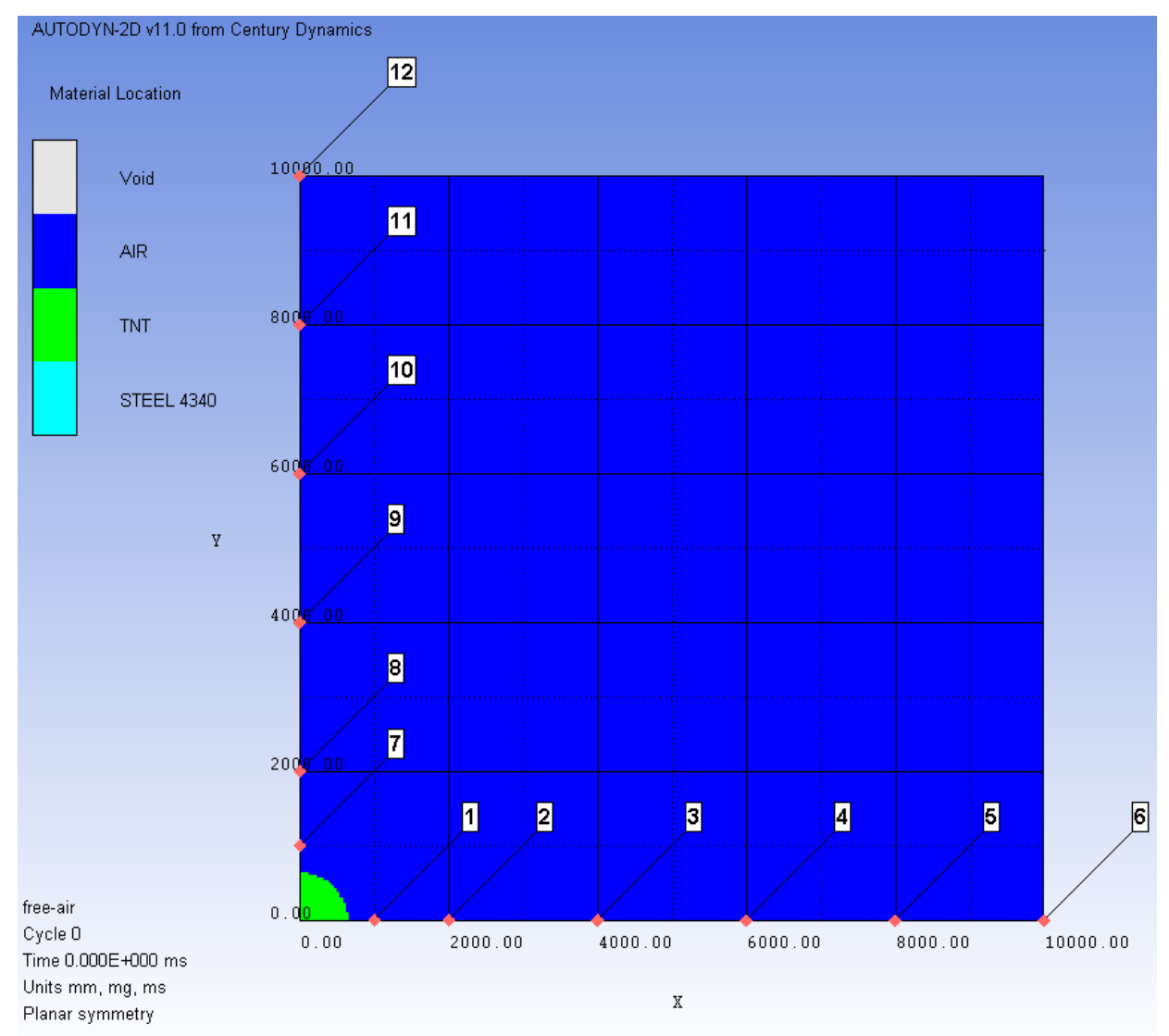

Figure 37: Location of Gauges in a free air model

Figure 38 shows the incident pressure values from a 20-kg TNT spherical charge obtained from AUTODYN model with different mesh sizes compared with peak incident pressure from ConWep. Figure 38 shows that the mesh size has more influence when the pressure is recorded at a closer distance to the center of charge. As the standoff distance increases, the effect of the mesh size is less. The dotted lines in the figure show the pressure value obtained from ConWep for different distances from the centre of detonation. From the figure, it can be seen that Mesh size of $5 \mathrm{~mm}$ yields reasonable results compared to those obtained from ConWep. A mesh size of $10 \mathrm{~mm}$ resulted in results about $2 \%$ lower peak incident pressure at $1 \mathrm{~m}$ but the computer run-time was substantially lower (40\% lower than for mesh size of $5 \mathrm{~mm}$ ). The mesh size of $10 \mathrm{~mm}$ was thus assumed to yield acceptable results for this specific blast problem. 


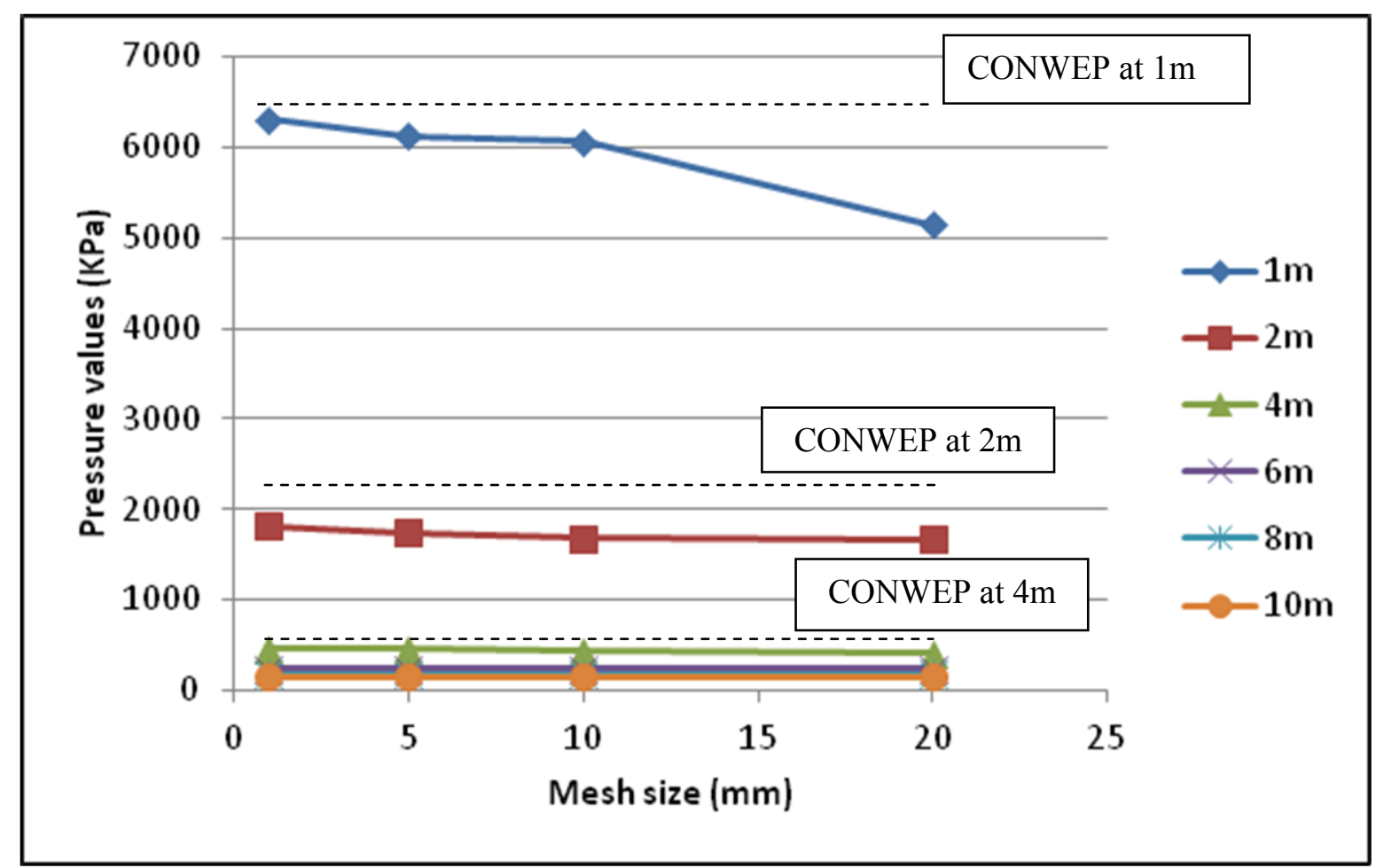

Figure 38: Sensitivity analysis

\subsection{Pressure attenuation of VSS}

When the blast wave encounters a vented object, a portion of the blast wave is reflected at the closed portion while the rest of the wave passes through the openings. This leads to the formation of wave jets in front of the VSS section. These jets form a zone of turbulence behind the VSS sections (Elshafey, 2009). The jets are then combined together to form a uniform pressure wave. From the studied cases, it was found that the waves combine again forming a uniform wave at a distance extending about $0.5-1.0 \mathrm{~m}$ from the VSS section depending on the charge mass and vent area ratio. Therefore, the pressure was recorded at a minimum distance of $2 \mathrm{~m}$ outside the container to avoid the turbulence zone that could affect the results. Figure 39 shows an example for a step by step interaction between the blast wave and a single layer VSS section. The figure shows the velocity vectors of the blast wave when it encounter a single layer VSS section forming the jets (Figure 39 (b)) and the combination of these jets again forming a uniform blast wave (Figure 39 (d)). 

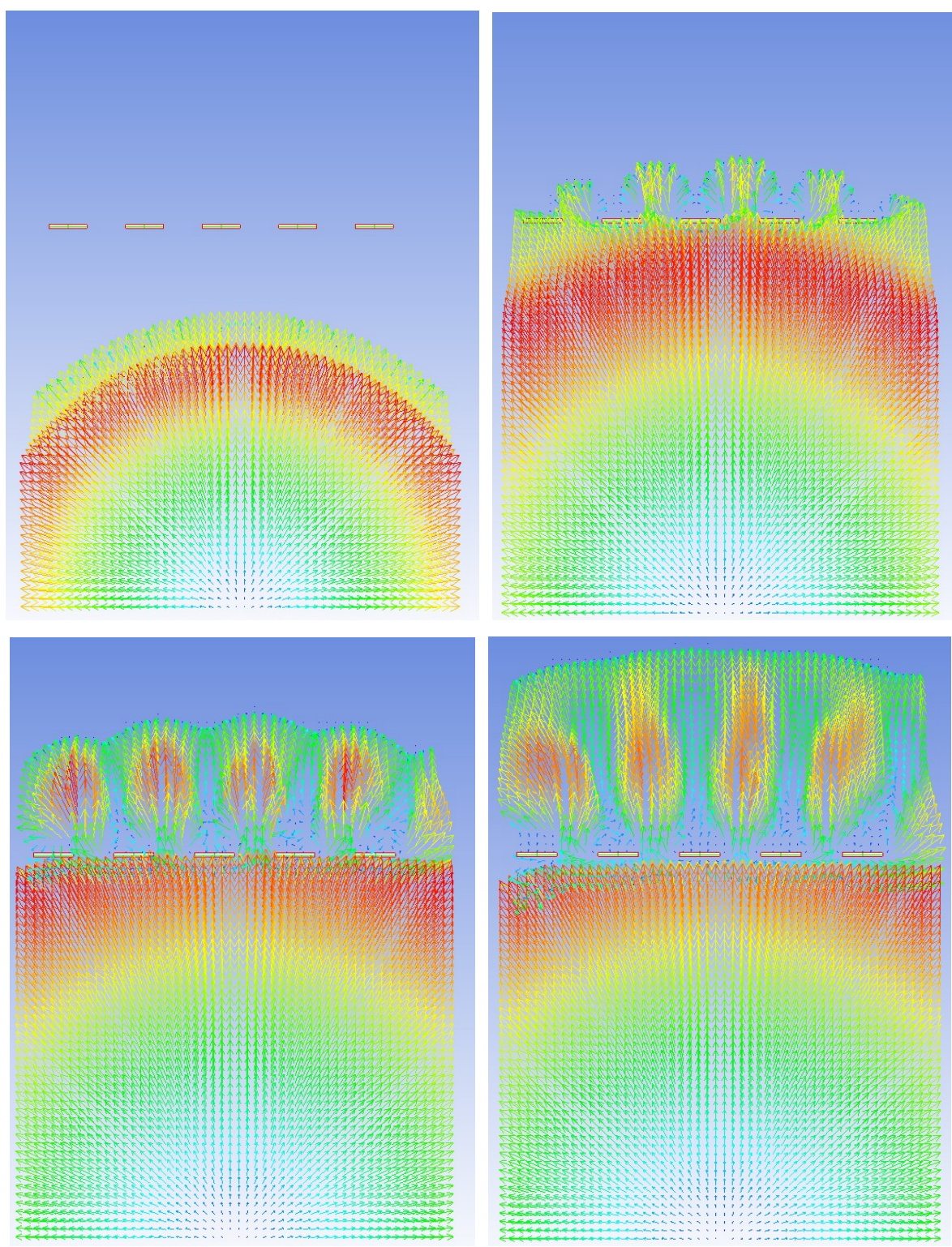

Figure 39: Velocity vectors at VSS location

\subsection{Factors affecting the pressure outside VSS containers}

From the literature review, there are three main factors that have a significant effect on the peak pressure outside VSS containers: charge mass, effective vent area ratio, and the container size. It is obvious that as the charge mass increase, the peak pressure outside the VSS container is higher. However, the effect of the other two parameters needs more studying to understand their effect on the peak pressure outside VSS containers. This section investigates the 
effect of effective vent area ratio and VSS container size on the peak pressure and impulse attenuation outside the container.

\subsubsection{Effect of vent area ratio}

The main inspiration behind having vents within the suppressive shield section is to allow some of the pressure to escape outside the container. As a result, it is expected that the section with a higher vents ratio will allow more pressure to escape outside and lead to lower peak pressure attenuation. Figure 40 shows the peak pressure outside a $(2.0 \times 2.0 \mathrm{~m})$ VSS container with different vent area ratios: $0.16,0.25,0.5$ and 0.75 due to an internal charge of $20 \mathrm{~kg}$ of TNT. The pressures are compared to that from a free field explosion with no shields.

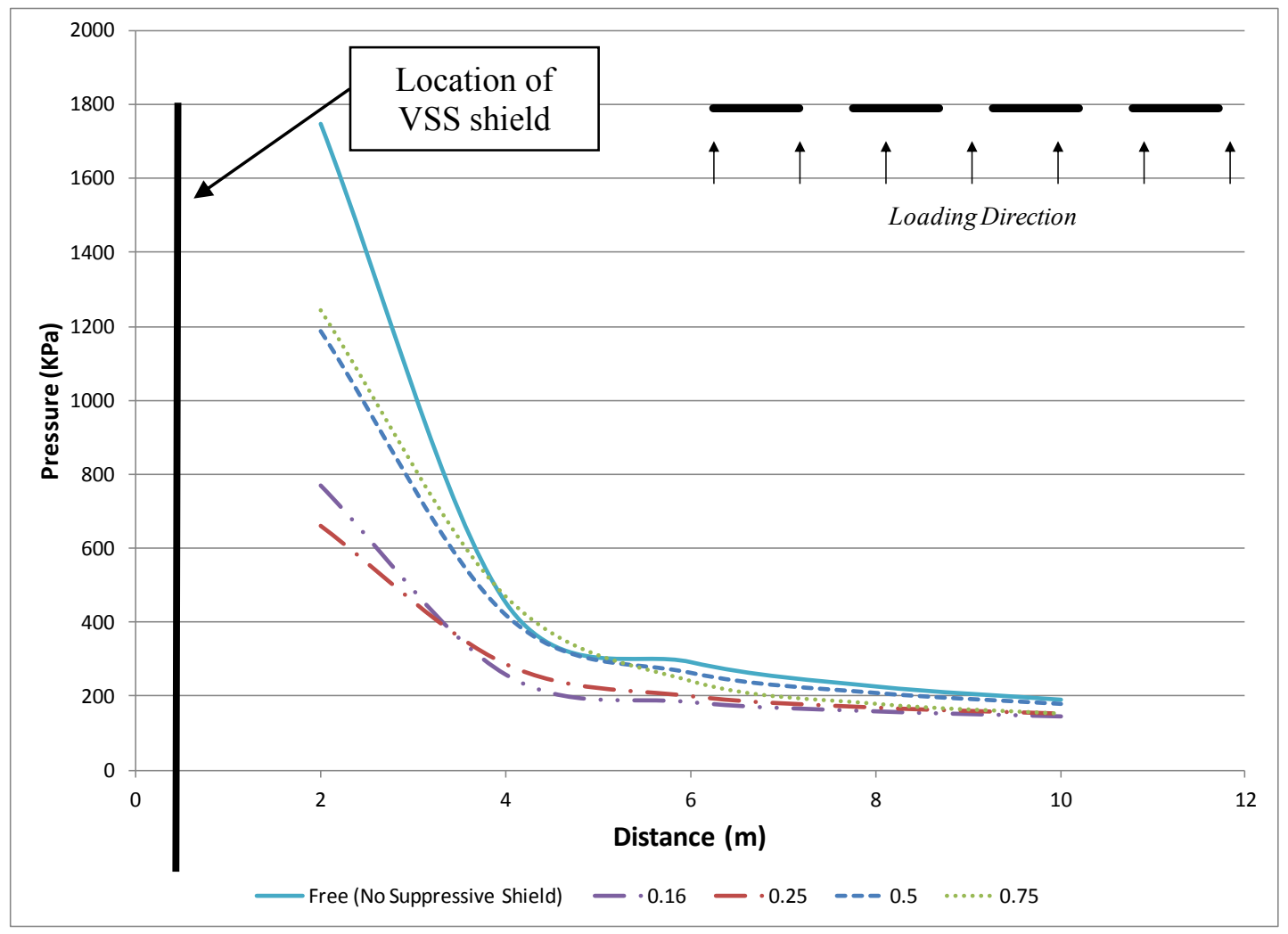

Figure 40: Effect of vent area ratio on the pressure outside

From Figure 40, it can be observed that the amount of vent area ratio of the VSS section controls how much pressure is released to the outside of the container. Thus the level of pressure attenuation for each VSS depends mainly on the vent area ratio. However, there is no relation 
that can be used to predict the performance of a VSS section given only the vent area ratio of this section.

\subsubsection{Effect of container size}

Different VSS containers with different container dimensions were modelled to study the effect of container size on the level of blast pressure attenuation. The container sizes that were modelled were $2.0 \times 2.0 \mathrm{~m}, 3.0 \times 3.0 \mathrm{~m}, 4.0 \times 4.0 \mathrm{~m}$. Figure 41 shows the relation between the calculated pressures outside the VSS container at different standoff distances: 4, 6, 8 and $10 \mathrm{~m}$ from the center of the container. This figure addresses a case of having a $20 \mathrm{~kg}$ TNT in the center of the container with 0.25 vent area ratio. In case of the $4.0 \times 4.0 \mathrm{~m}$, the pressure was not calculated at $2 \mathrm{~m}$ from the center of detonation because at that distance is the wall of the VSS container so the pressure in this case was tracked at $4,6,8$ and $10 \mathrm{~m}$ from the center of detonation.

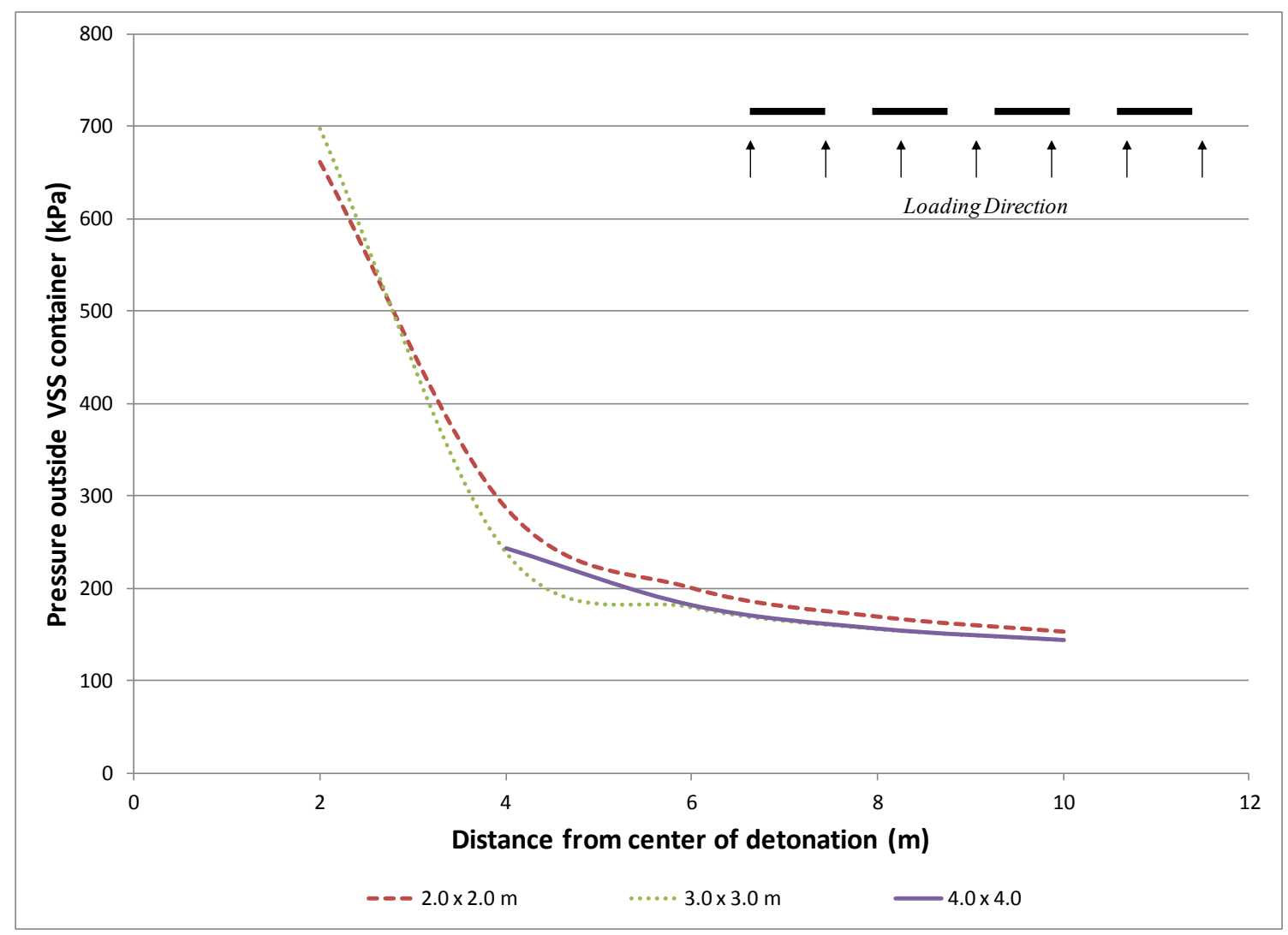

Figure 41: Effect of container size on the pressure outside the container 
It can be observed from the figure that the size of the container does not have an efficient effect on the pressure outside. This may be explained by the pressure released through the vents in the suppressive shield section no matter how far the side of the container is from the charge inside. However, the size of the container affects the pressure inside the container as will be discussed later in the next chapter.

\subsubsection{Multi-layer VSS configurations}

For multi-layer VSS configurations, the capacity to attenuate blast pressure is expressed by the effective vent area ratio, $\alpha_{e}$. The effective vent ratio is calculated using Equation 2 mentioned earlier in Chapter 2.

The effect of distance between layers in the double-layer plate configuration, $g$, was investigated by varying the ratio of the gap between the layers of the VSS section $g$ to the gap between the plates $a$. So the effect of $g / a$ on the level of attenuation of double-layer plate VSS section is investigated. Figure 42 presents the incident peak pressure values outside $2.0 \times 2.0 \mathrm{~m}$ VSS containers with an internal charge of $20 \mathrm{~kg}$ of TNT. The effective vent area ratio of the studied VSS sections were all equal to 0.2 , however, the ratio $g / a$ varied between 0.25 and 2.0. The double layer VSS sections were compared to another single layer VSS section with the same vent area ratio equals to 0.2 . 


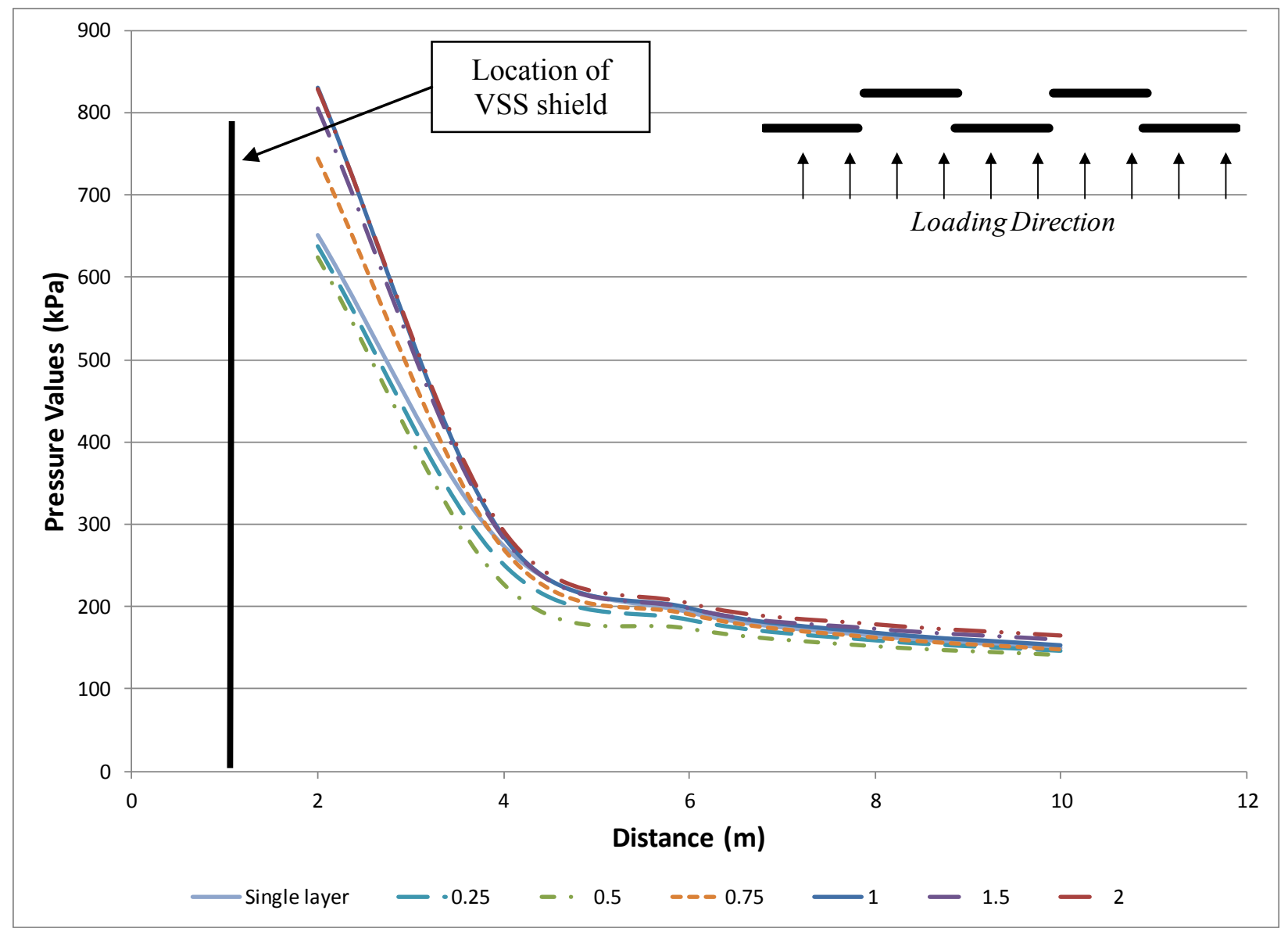

Figure 42: Effect of $g / a$ ratio on peak incident pressure outside VSS container (20 kg TNT charge)

Figure 42 shows that the for double layer VSS sections with $\mathrm{g} / \mathrm{a}$ ratios of equal to 0.25 and 0.5 , the attenuation of blast loads was the same at different distances as for the single-layer plate VSS with the same vent area ratio. But for other double layer VSS sections with $g / a$ ratios of 0.75 and more, the attenuation was different. Thus Equation 2 proposed by (Cox et al., 1978) is valid for multi-layer VSS configurations for $g / a$ ratios less than 0.5 . Peak incident pressure values were observed to be higher at the scaled distances considered when the g/a ratio is greater than or equal to 0.5 .

Equation 2 proposed by Cox et al (1978), was also validated for multi-layer plate configurations consisting of 1,2,3 and 4 single-layer plate configurations with effective vent area ratio of 0.16 and 0.20 . The peak pressures at different distances from the VSS container were measured for different TNT charges: 10 and $20 \mathrm{~kg}$. Figure 44 and Figure 45 show the 
relation between the pressure calculated outside the containers verses scaled distance for 5, 10 and $20 \mathrm{~kg}$ TNT, respectively. These curves were plotted to investigate the level of attenuation of VSS with 0.16 vent area ratio with respect to scaled distances.

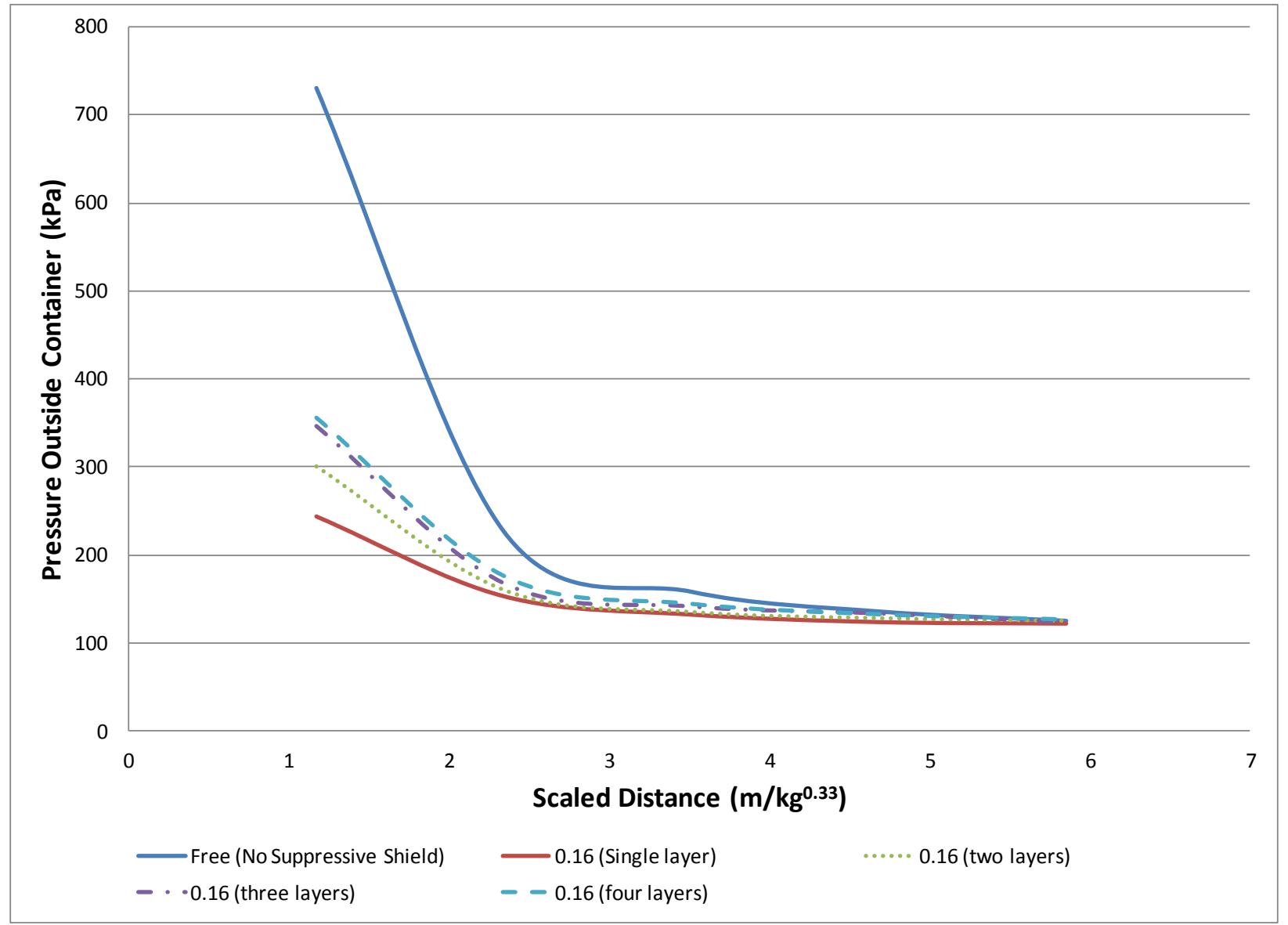

Figure 43: Relation between Pressure values and scaled distance for VSS section with 0.16 vent area ratio $($ TNT charge $=5 \mathrm{~kg})$ 


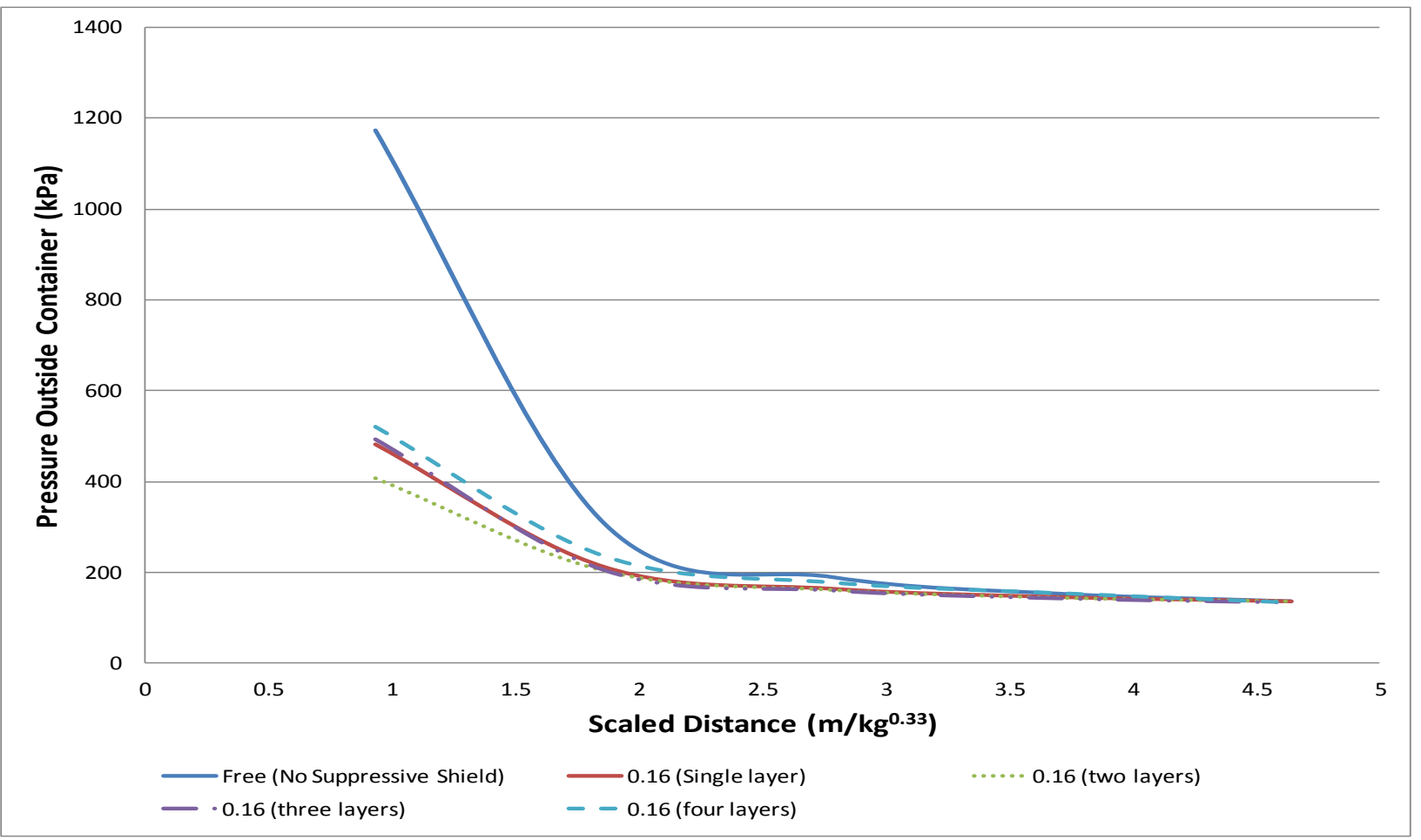

Figure 44: Relation between Pressure values and scaled distance for VSS section with 0.16 vent area ratio ( TNT charge $=10 \mathrm{~kg}$ )

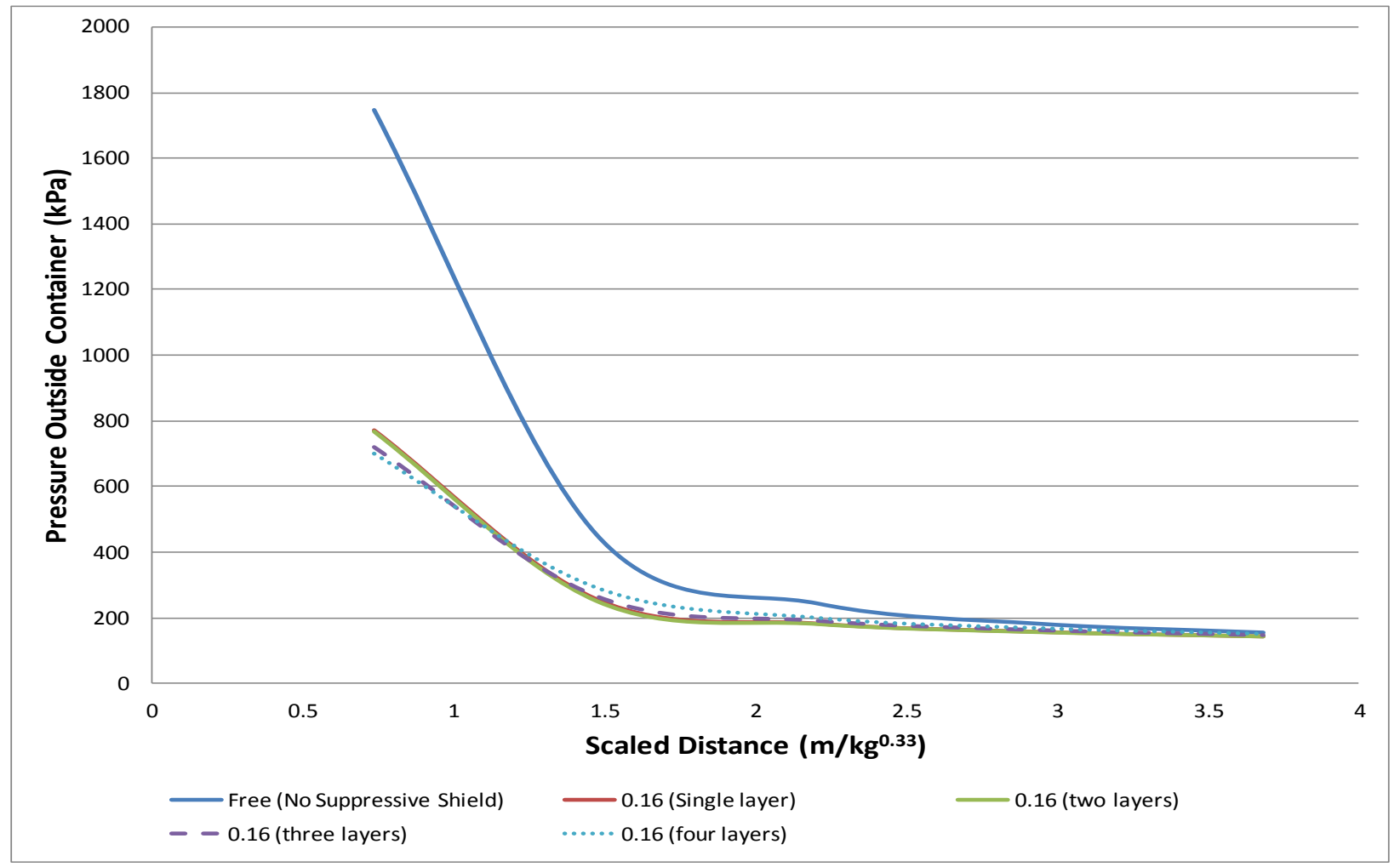

Figure 45: Relation between Pressure values and scaled distance for VSS section with 0.16 vent area ratio ( TNT charge $=20 \mathrm{~kg}$ ) 
From the three figures, it can be observed that at scaled distance $2.5\left(\mathrm{~m} / \mathrm{kg}{ }^{0.33}\right)$, the effect of having a VSS section didn't have a sufficient effect on the blast load pressure outside. This conclusion could be used in defining the Q-D required outside a given container with a given TNT charge inside. Same curves were plotted for VSS sections with 0.2 vent area ratio and this is shown in Figure 46, Figure 47 and Figure 48.

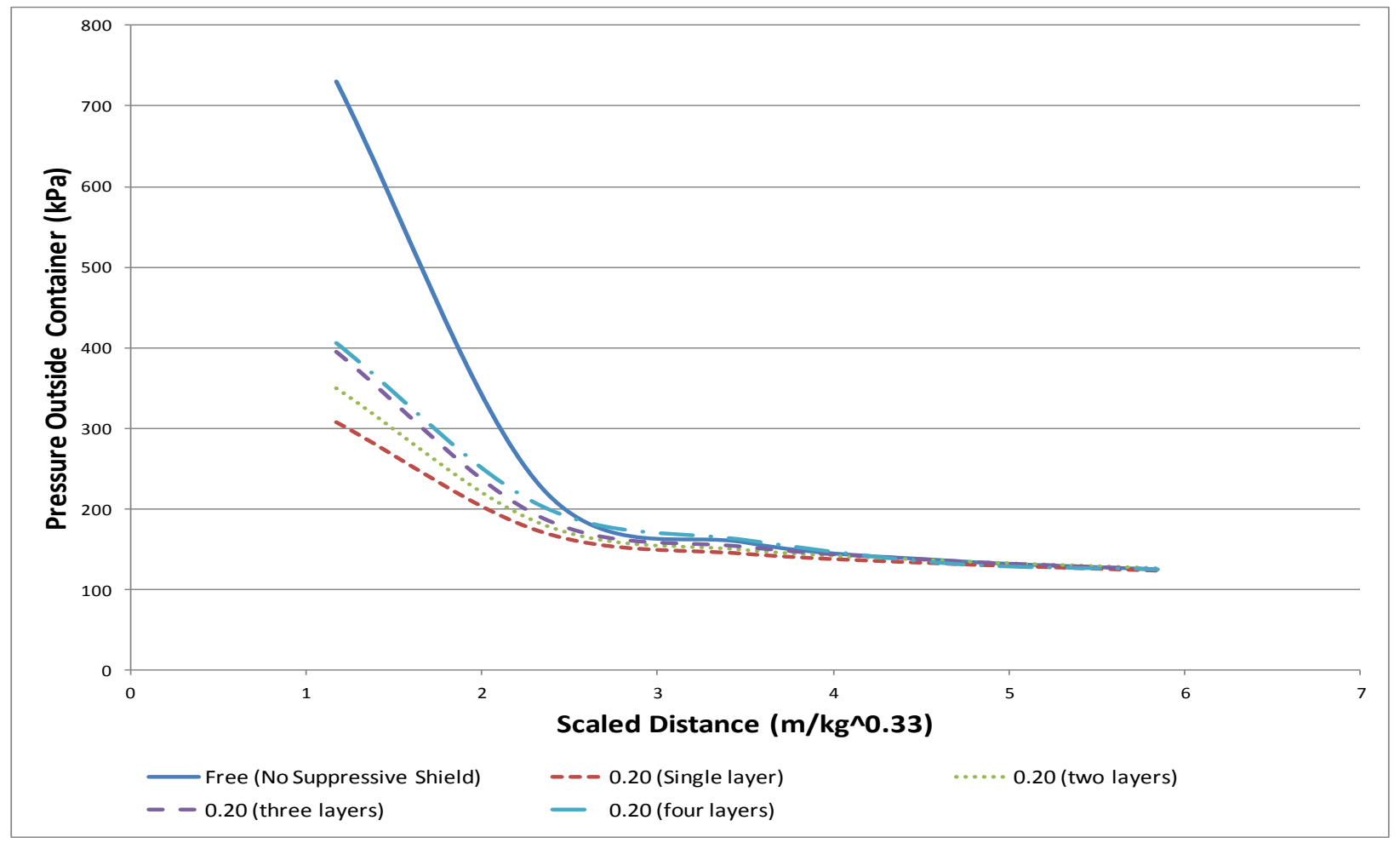

Figure 46: Relation between Pressure values and scaled distance for VSS section with 0.20 vent area ratio $($ TNT charge $=5 \mathrm{~kg})$ 


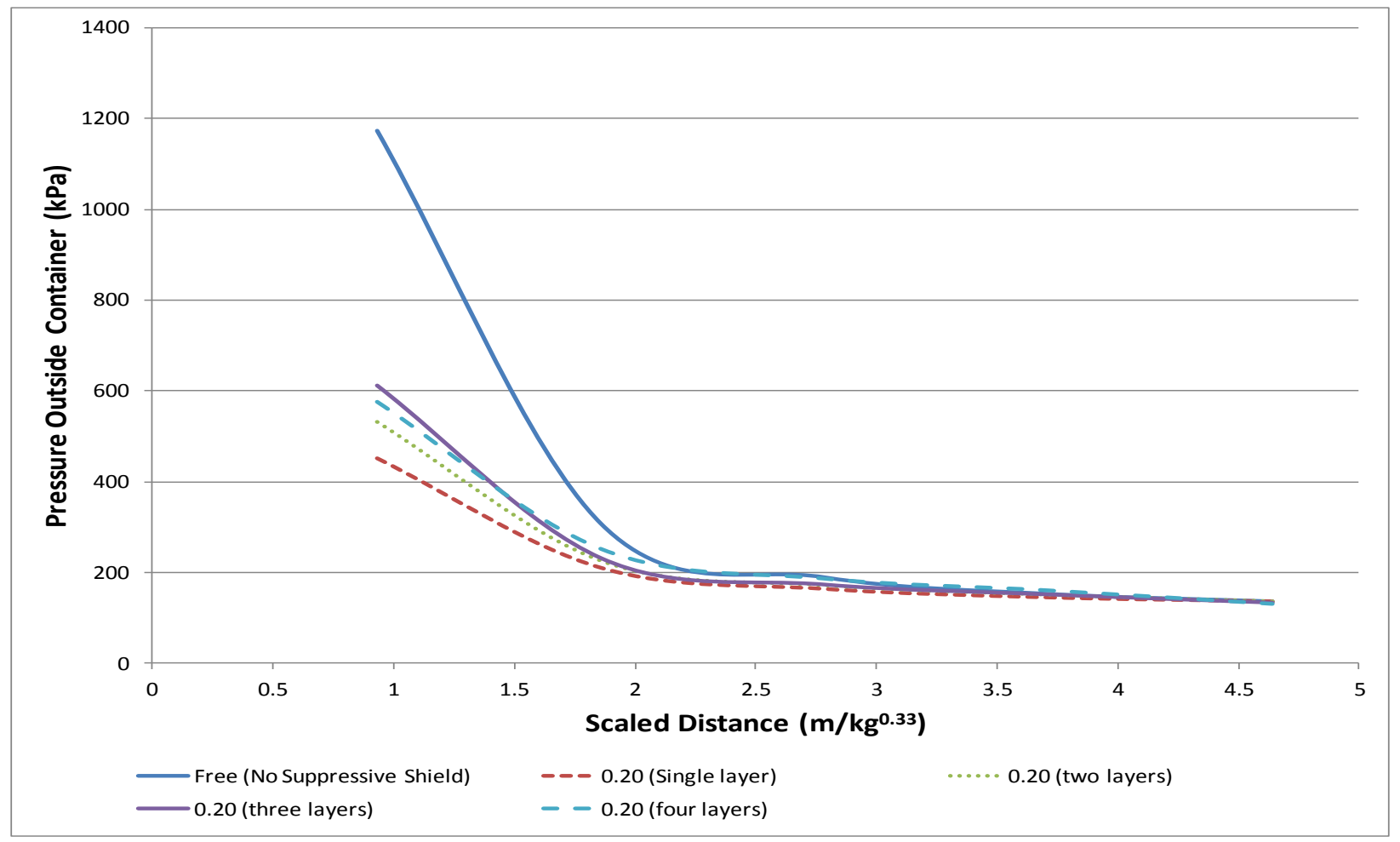

Figure 47: Relation between Pressure values and scaled distance for VSS section with 0.20 vent area ratio ( TNT charge $=10 \mathrm{~kg}$ )

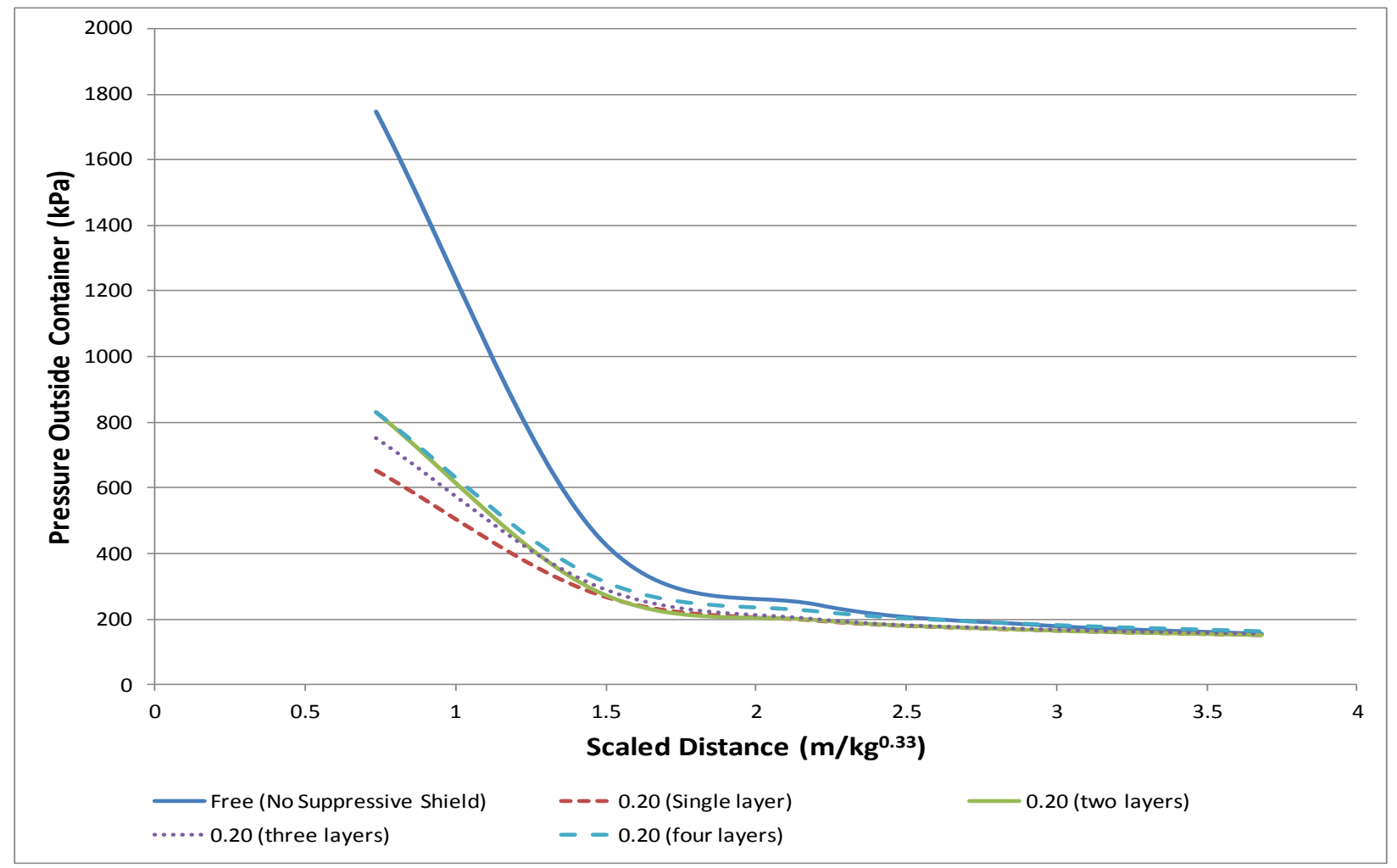

Figure 48: Relation between Pressure values and scaled distance for VSS section with 0.20 vent area ratio ( TNT charge $=20 \mathrm{~kg}$ ) 
By studying the three curves, it can be observed that the same phenomenon that was observed in case of the 0.16 VSS sections was also found in case of 0.20 VSS section. The level

of attenuation drops to zero after reaching a scaled distance equals to $2.5\left(\mathrm{~m} / \mathrm{kg}^{0.33}\right)$. At this point having a VSS or not won't have a sufficient effect on the attenuation of blast loads.

\subsection{Comparison between VSS configurations}

The suppressive shield configurations presented in the previous sections were all modeled in AUTODYN to investigate the effect of the geometric configuration configurations on the blast pressure and impulse attenuation outside the VSS container. Comparisons were completed for suppressive shields with vent area ratios (effective vent area ratios) of 0.25 . The size of the VSS container was maintained constant $(2.0 \times 2.0 \mathrm{~m})$. Three TNT charge masses: 5,10 and $20 \mathrm{~kg}$ were used in the comparison. The vent area ratio for each configuration was calculated by using Equation 23, Equation 24 and Equation 25. For the inclined plate VSS configuration, the plates were inclined at $20^{\circ}$ to obtain a vent ratio equals to 0.25 using Equation 24 mentioned earlier in this chapter.

The incident blast pressure from the explosion in the VSS containers was monitored outside the container from $2.0-10.0 \mathrm{~m}$ as well as the impulse. The incident blast pressure and incident impulse in the first $1.0 \mathrm{~m}$ outside the VSS container was not investigated because of turbulence and blast wave jetting in this region (Elshafey, 2009). Table 7 shows the properties of different VSS configuration for (effective) vent area ratio of 0.25 . 
Table 7: Properties of different VSS shields with the same vent ratio 0.25

\begin{tabular}{|c|c|c|c|c|c|c|}
\hline VSS section & Geometric configuration & $\begin{array}{l}\text { Length } \\
\text { of } \\
\text { shield } \\
(\mathrm{mm})\end{array}$ & $\begin{array}{c}\text { Gap } \\
\text { between } \\
\text { plates }(\mathrm{mm})\end{array}$ & $\begin{array}{l}\text { Angle of } \\
\text { inclination }\end{array}$ & $\begin{array}{l}\text { Number of } \\
\text { elements/m } \\
\text { (n) }\end{array}$ & $\begin{array}{l}\text { Vent } \\
\text { ratio }\end{array}$ \\
\hline $\begin{array}{l}\text { Single layer } \\
\text { plates }\end{array}$ & & 150 & 50 & 0 & 5 & 0.25 \\
\hline $\begin{array}{l}\text { Single layer } \\
\text { Inclined plates }\end{array}$ & & 150 & 144 & $20^{*}$ & 7 & 0.25 \\
\hline $\begin{array}{l}\text { Single-layer } \\
\text { side-by-side } \\
\text { angle exterior- } \\
\text { facing }\end{array}$ & & 106 & 50 & 45 & 5 & 0.25 \\
\hline $\begin{array}{l}\text { Single-layer } \\
\text { side-by-side } \\
\text { angle interior- } \\
\text { facing }\end{array}$ & & 106 & 50 & 45 & 5 & 0.25 \\
\hline $\begin{array}{l}\text { Single layer } \\
\text { Nested angles }\end{array}$ & & 150 & 19 & 45 & 53 & 0.25 \\
\hline \multirow{2}{*}{$\begin{array}{l}\text { Double layer } \\
\text { plates }\end{array}$} & & 100 & 100 & 0 & 5 & \multirow{2}{*}{0.25} \\
\hline & & 100 & 100 & 0 & 5 & \\
\hline \multirow{2}{*}{$\begin{array}{l}\text { Double-layer } \\
\text { nested side- } \\
\text { by-side angles }\end{array}$} & & 150 & 100 & 45 & 3 & \multirow{2}{*}{0.25} \\
\hline & & 150 & 100 & 45 & 3 & \\
\hline
\end{tabular}

* The 20 degrees was selected to obtain a section with 0.25 vent area ratio according to Equation 24

Figure 49 and Figure 50 present the pressure and impulse values outside VSS containers with the configurations presented in Table 1. The inclined single-layer plate configuration exhibited the lowest peak incident pressure outside the VSS container while the exterior-facing side-by-side angle configuration exhibited the highest peak pressures (Figure 49). The doublelayer nested side-by-side angle configuration had the same peak pressure outside the VSS section as nested single-layer plate configuration. The double-layer nested side-by-side angle configuration produced the lowest incident impulse at smaller distances from the VSS container while the single-layer nested angle configuration produced the lowest incident impulse at larger 
distances from the VSS side wall. The angle of inclination of the single-layer inclined plate and single-layer nested angle configurations results in reflection and direction change of the blast wave leading to incident pressure and impulse reduction. The single-layer side-by-side angle configurations (exterior-facing and interior-facing) have openings that allow a direct path for the pressure propagation to the outside the container. Thus their blast pressure and impulse attenuation capacity is marginal in comparison to the single-layer plate configuration.

The single-layer side-by-side angle configuration (interior facing) serves to reflect blast pressure waves towards the centre of the VSS container thereby limiting the amount of pressure to the outside. This could lead to high pressure loading on the VSS elements. The single-layer side-by-side angle configuration (exterior facing) on the other hand reflects blast pressure waves towards the outside and thus could lead to lower pressure loading on the VSS elements in comparison to the interior facing configuration.

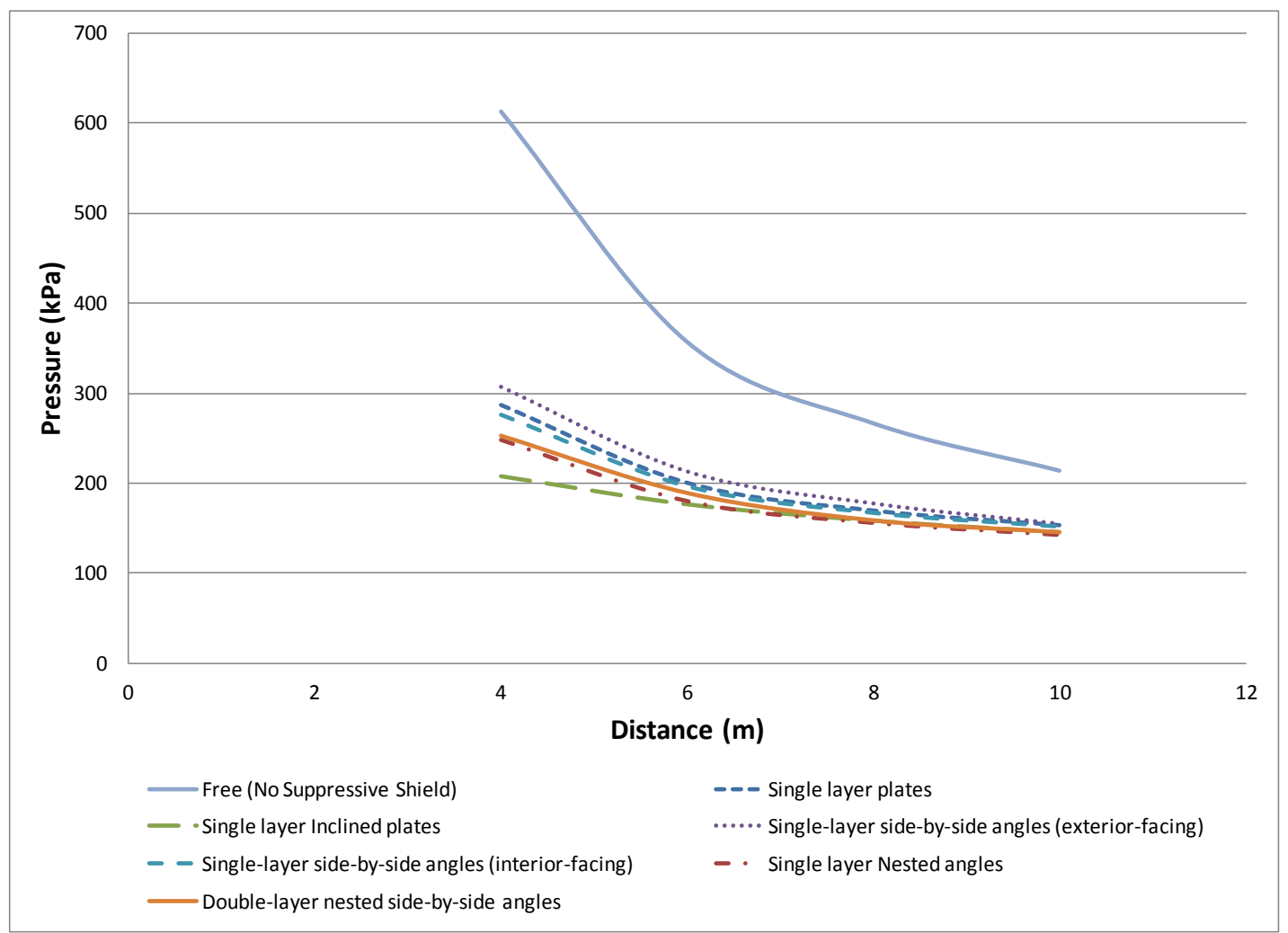

Figure 49: Pressure values for different geometric configurations with 0.25 vent ratios 


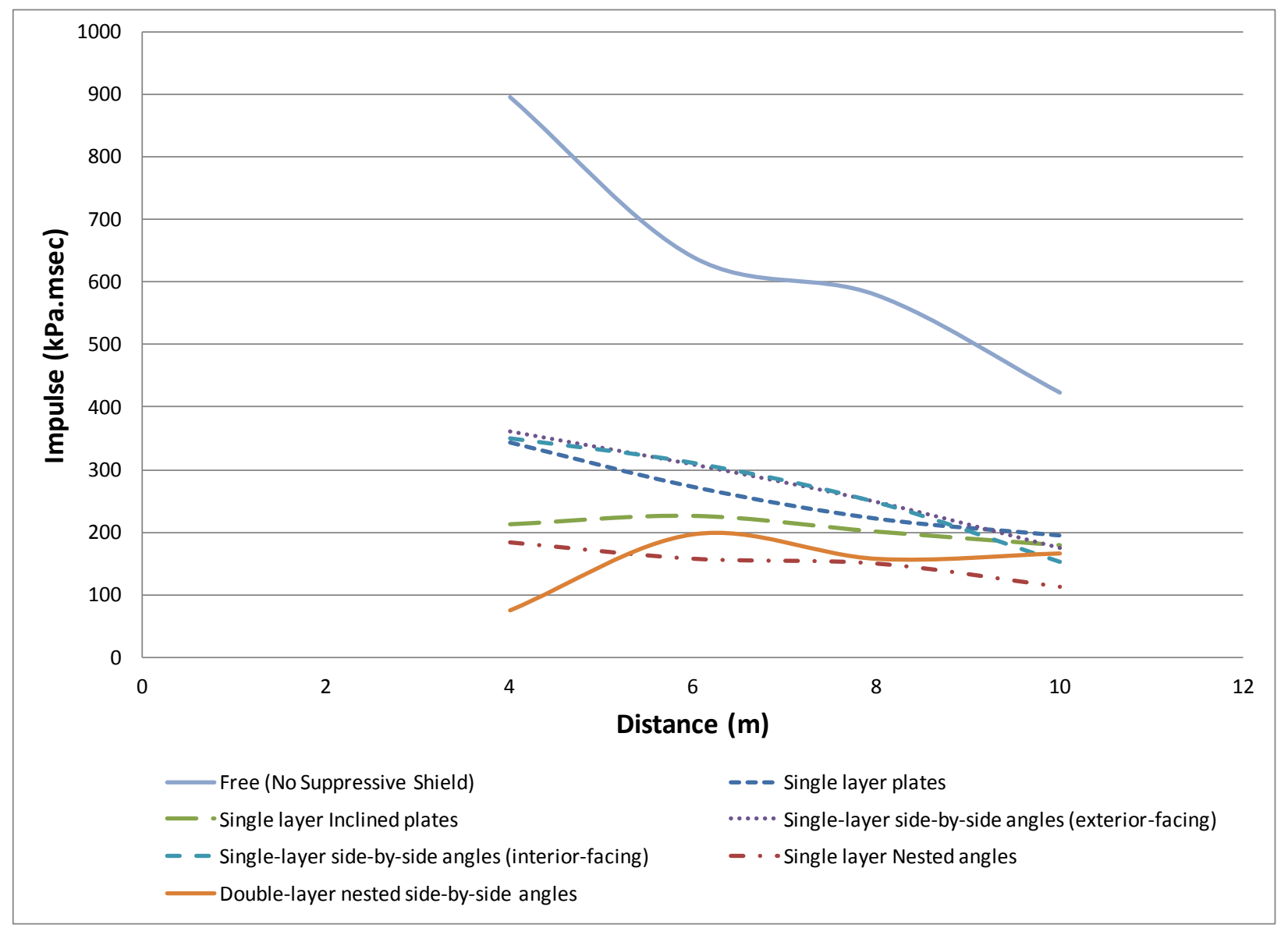

Figure 50: Impulse values for different geometric configurations with 0.25 vent ratios

\subsection{Prediction of blast pressure outside vented structures}

VSS structures comprise of single-layer or multi-layer structural steel panels connected to edge frame elements. A typical VSS layer provides venting of blast pressure from the container and at the same time attenuates the pressure outside the container. The level of blast pressure attenuation depends on the geometric configuration and effective vent area ratio of the container. Several configurations of VSS have been proposed in the literature and investigations have shown a strong correlation between the level of attenuation of blast pressure and impulse and vent area ratio. However, no rational or theoretically based equation exists for calculating the vent area ratio (Cox et al., 1978). Computing the vent area ratio for VSS containers presents a big challenge due to the large variety of geometric configurations possible. Guidance on calculating the vent area ratio for a few VSS containers was published by the US Army (Cox et al., 1976). 
A number of relationships have been proposed by previous researchers to calculate the pressure outside VSS sections. However, these equations have limitations to their range of applicability. The variables that were considered to affect the pressure value outside were the charge weight, effective vent ratio and size of the container. Esparza et al. (1975) proposed Equation 26 and Equation 27 to predict the peak blast pressure and impulse outside VSS, respectively.

$$
\begin{array}{r}
P_{s}=957 Z^{-1.66}\left(\frac{R}{X}\right)^{0.27} \alpha_{\text {eff }}^{0.64} \\
\qquad=\frac{R}{\sqrt[3]{W}}
\end{array}
$$

Equation 26

Where $W$ is the weight of TNT (lbs.), $R$ is stand-off distance, distance between the charge and the point at which the pressure is recorded (ft.), $X$ is the length of side of the container (ft.), $\alpha_{\text {eff }}$ is effective vent area ratio and $\mathrm{Z}\left(\mathrm{ft} / \mathrm{lbs}^{(0.33)}\right)$ is the scaled distance. The proposed Equation 26 is valid for the following limits:

$$
\begin{aligned}
& 2.93<Z<21.3 \\
& 0.69<\frac{R}{X}<4.55 \\
& 0.01<\alpha_{\text {eff }}<0.13
\end{aligned}
$$

Impulse is defined as the area under the pressure-time curve. The impulse value indicates how long the applied load has been acting on the point of study. Esparza et al. (1975) proposed Equation 27 to predict the impulse value outside vented structures.

$$
I_{s}=\left[218\left(\frac{1}{Z}\right)^{0.98}\left(\frac{R}{X}\right)^{0.008} \alpha_{\text {eff }} 0.45\right] \sqrt[3]{W}
$$

Equation 27

The impulse Equation 27 is valid within the following limits:

$$
\begin{aligned}
& 2.93<Z<15 \\
& 1.16<\frac{R}{X}<4.55 \\
& 0.008<\alpha_{\text {eff }}<0.13
\end{aligned}
$$


Another Equation 28 proposed by Baker (1975) to calculate the blast pressure outside vented structures. The equation had the same variables used by Esparza et al. (1975); however, the governing factors were expressed differently. No equation to calculate the impulse was proposed in the study by Baker (1975).

$$
P_{S}=976.3 \frac{W^{\frac{2}{3}} \alpha^{\frac{1}{2}}}{R^{\frac{3}{2}} X^{\frac{1}{2}}}
$$

And the limits to use this equation are as follows:

$$
\begin{aligned}
& 4.27<Z<17.5 \\
& 0.323<\frac{X}{R}<1.77 \\
& 0.026<\alpha_{\text {eff }}<0.6
\end{aligned}
$$

The literature review has revealed very little work in the area of blast pressure mitigation using suppressive shields. The amount of work available is in reports to the US Army and are primarily meant for explosive storage where the structures can be massive. Also, there are no rationale equations for determining the vent area ratio of VSS configurations other than those presented by Baker (1975).

\subsubsection{Prediction of blast pressure and impulse outside VSS containers}

In order to obtain a good understanding for the factors affecting the pressure and impulse outside VSS container, ninety (90) models with different VSS configurations, container size, and charge mass were analyzed. The analyses results were compared with blast pressure and impulse equations proposed in the literature. Figure 51 presents a comparison between the results obtained from AUTODYN and from Equation 26 and Equation 28. Figure 51 shows that the equations in the literature under predicts the blast pressure in comparison to AUTODYN numerical results. 


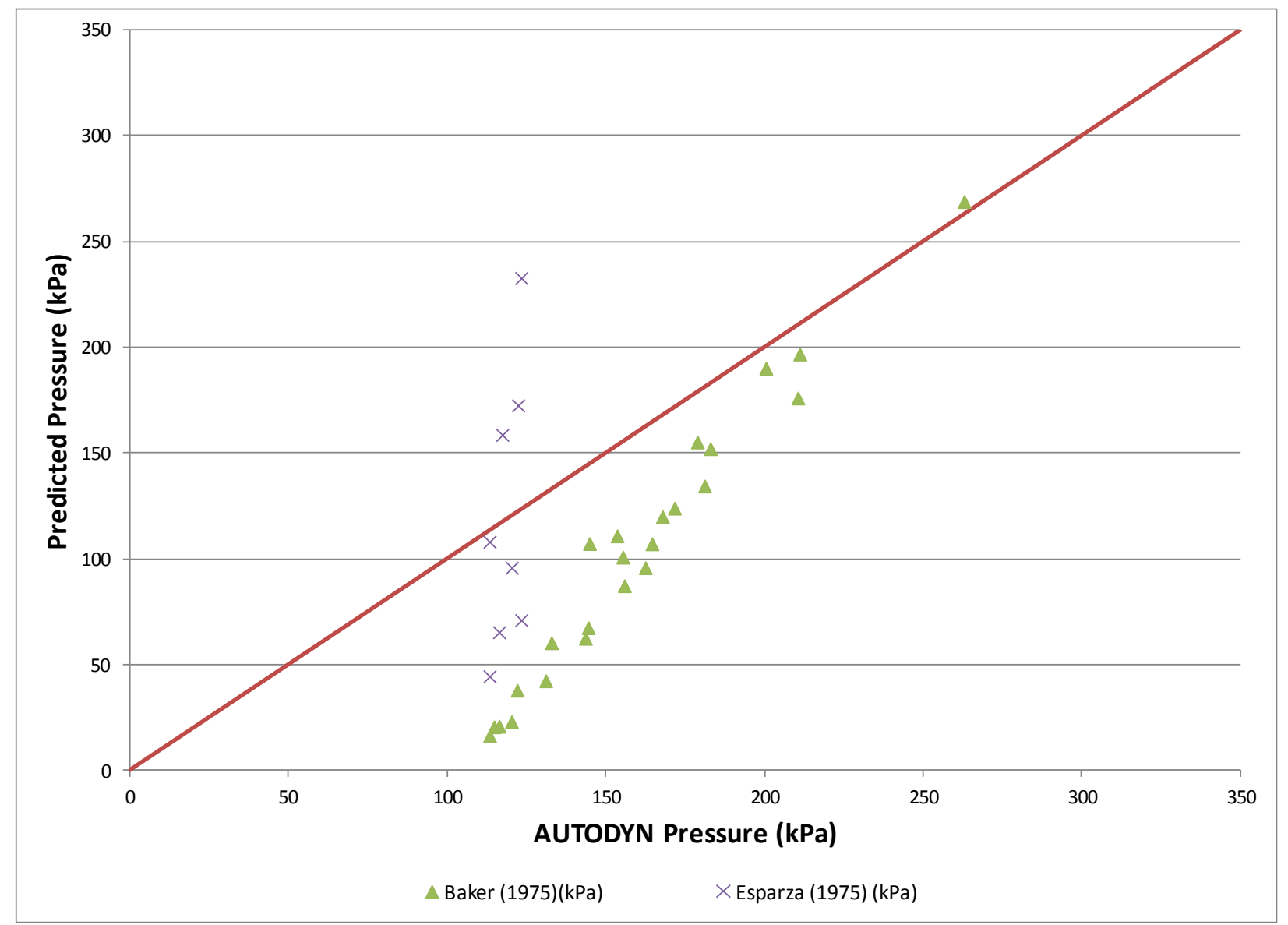

Figure 51: Comparison between available equations and numerical results (AUTODYN)

In order to improve ability to predict blast pressure attenuation of suppressive shields, a regression analysis was carried out on the results from the 90 AUTODYN models to identify variables affecting the blast pressure and impulse outside the VSS container. The variables investigated included the amount of explosive charge, the (effective) vent area ratio, the size of the container, and the standoff distance at which the pressure and impulse are required to be measured. Equation 29 is proposed for calculating the peak incident blast pressure is at a given standoff distance outside the VSS container.

$$
P_{s}=310 \frac{W^{0.2} \alpha_{e f f}^{0.06}}{R^{0.5} X^{0.1}}
$$

Equation 29

Where, $W$ is TNT charge weight $(\mathrm{kg}), R$ is standoff distance $(\mathrm{m}), X$ is length of side of square VSS container $(\mathrm{m})$, and $\alpha_{\text {eff }}$ is the effective vent area ratio. 
The charge weights investigated varied between 1 to $20 \mathrm{~kg}$ of TNT, the effective vent area ratios between 0.16 and 0.75 , and VSS containers size of $2.0 \times 2.0,3.0 \times 3.0$ and $4.0 \times 4.0 \mathrm{~m}$. The peak incident blast pressure and impulse were monitored outside the VSS container up to a standoff distance of $10 \mathrm{~m}$. Thus the equation developed from the regression analysis is assumed valid within the following limits.

$$
\begin{aligned}
& 1.33<Z<20 \\
& 0.1<\frac{X}{R}<1.33 \\
& 0.02<\alpha_{\text {eff }}<0.75
\end{aligned}
$$

Impulse was obtained from the integration of the pressure time history curves from the AUTODYN models. These values were used to develop the proposed Equation 30 for incident impulse outside the VSS containers.

$$
I_{s}=758.5 \frac{W^{0.14} \alpha_{e f f}^{0.69}}{Z^{0.58}(X / R)^{0.55}}
$$

\section{Equation 30}

The proposed equation had some limits that need to be satisfied to be valid for calculation of the impulse. These limits are different than the limits required for the pressure equation. The limits are given as below:

$$
\begin{aligned}
& 3.7<Z<20 \\
& 0.0<\frac{X}{R}<1.0 \\
& 0.15<\alpha_{\text {eff }}<0.75
\end{aligned}
$$

Figure 52 and Figure 53 present the comparison of peak incident pressure and incident impulse obtained from AUTODYN analyses and by using the equations proposed in this thesis. The figures show good correlation between the peak incident pressure and incident impulse from the AUTODYN analyses and with the proposed equation, as expected. 


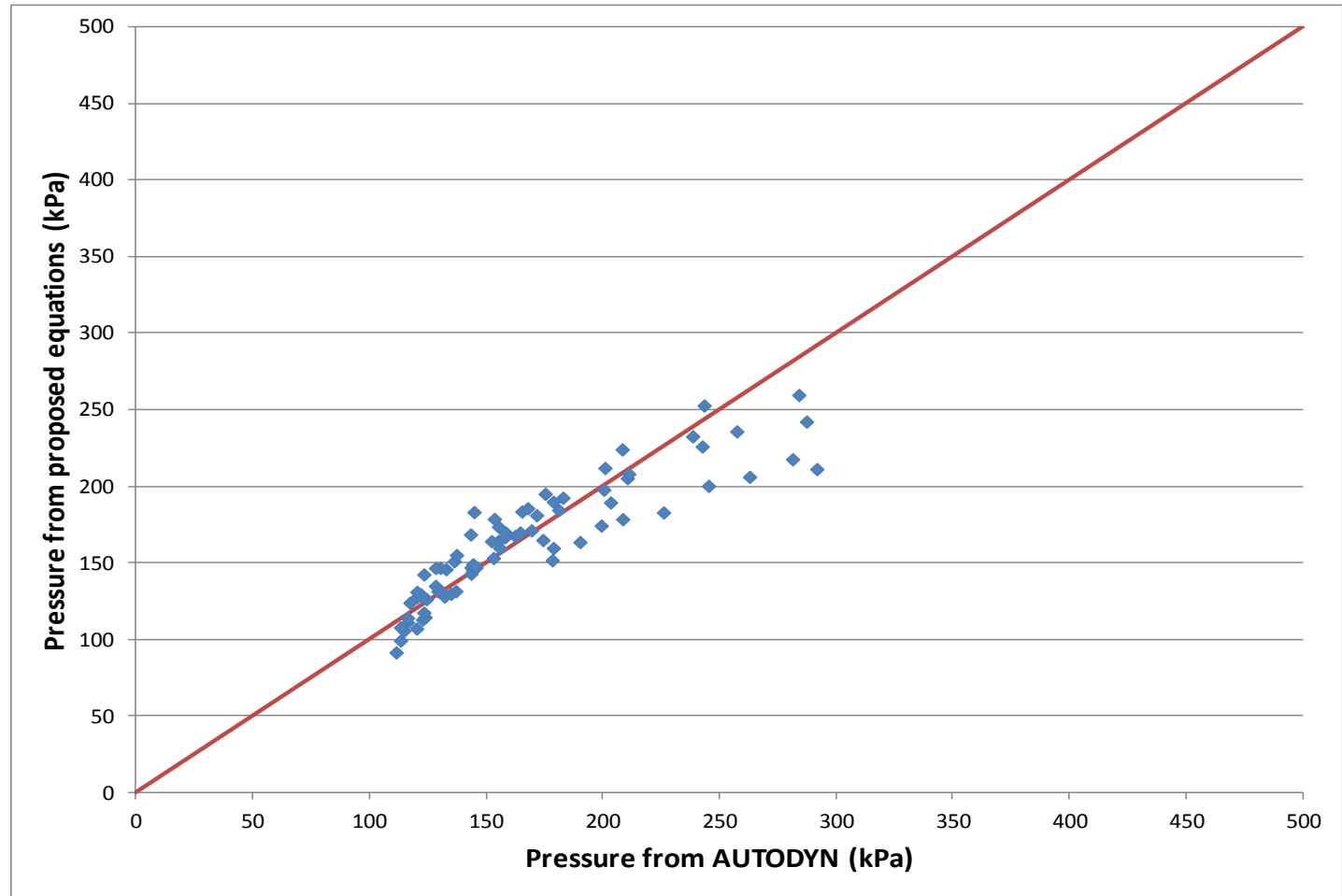

Figure 52: Comparison between obtained Pressure values from proposed equation and numerical results

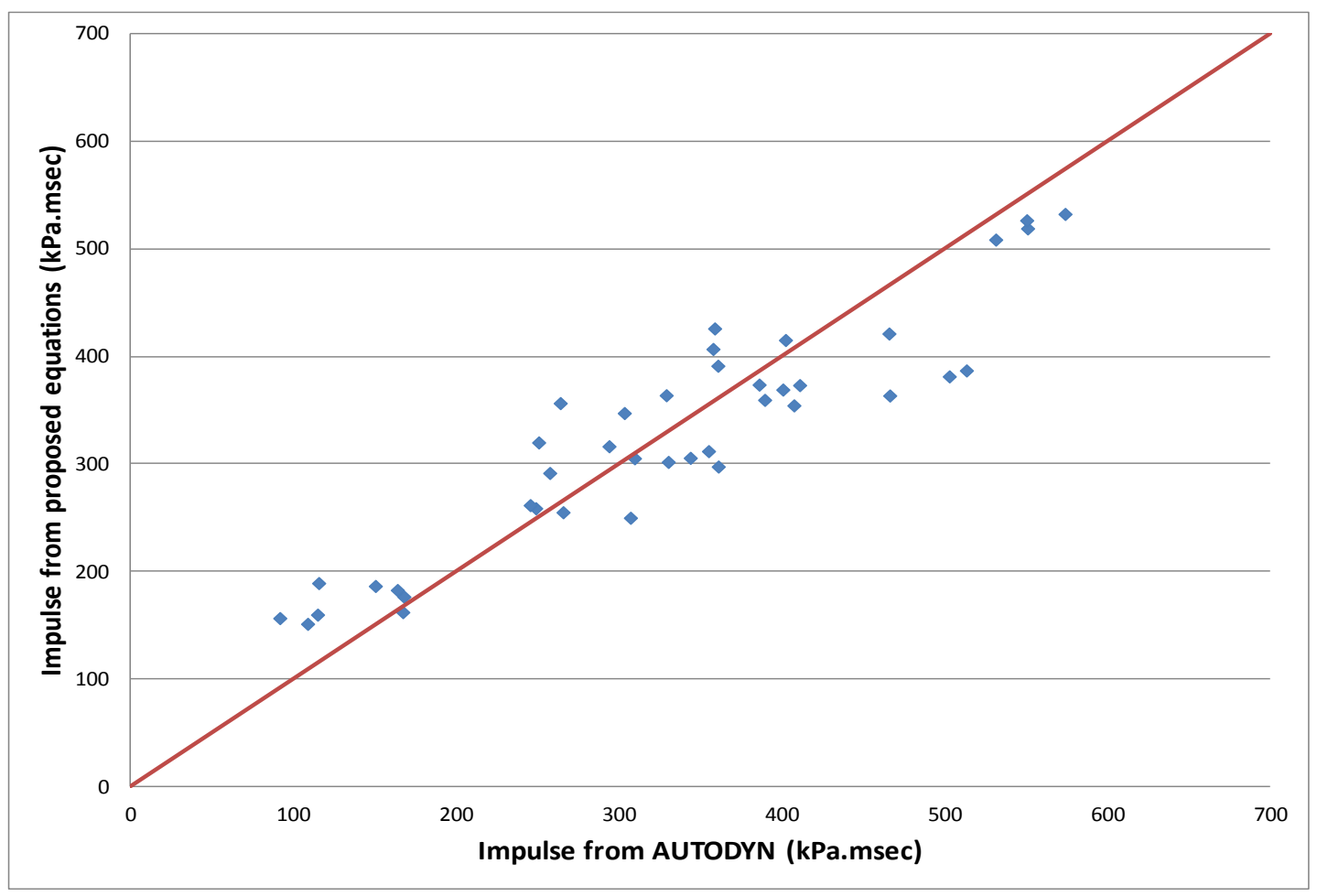

Figure 53: Comparison between obtained Impulse values from proposed equation and numerical results 


\subsubsection{Verification of proposed equation}

The proposed Equation 29 was validated with experimental results from a previous experimental research program (Elshafey, 2008). Elshafey carried out experimental testing using VSS panels with four different geometric configurations (see Figure 19), three different effective vent area ratios and three different Pentolite charge masses: $0.25,0.5$ and $1 \mathrm{~kg}$. For SSP-A the vent area ratio was 0.13 , SSP-B and SSP-D were 0.02 and for the SSP-C configuration, the vent area ratio was 0.08 . The pressure was tracked at different locations outside the VSS section: 1.5 , 2.5 and $3.5 \mathrm{~m}$ from the center of detonation while the VSS section was placed at $1.0 \mathrm{~m}$ from the center of detonation. The results obtained by Elshafey for all the four geometric configurations in this study were compared to the results obtained from the proposed equations. Figure 54 shows a comparison between the pressure values obtained from the developed Equation 29 and those obtained from the experimental work (Elshafey, 2008).

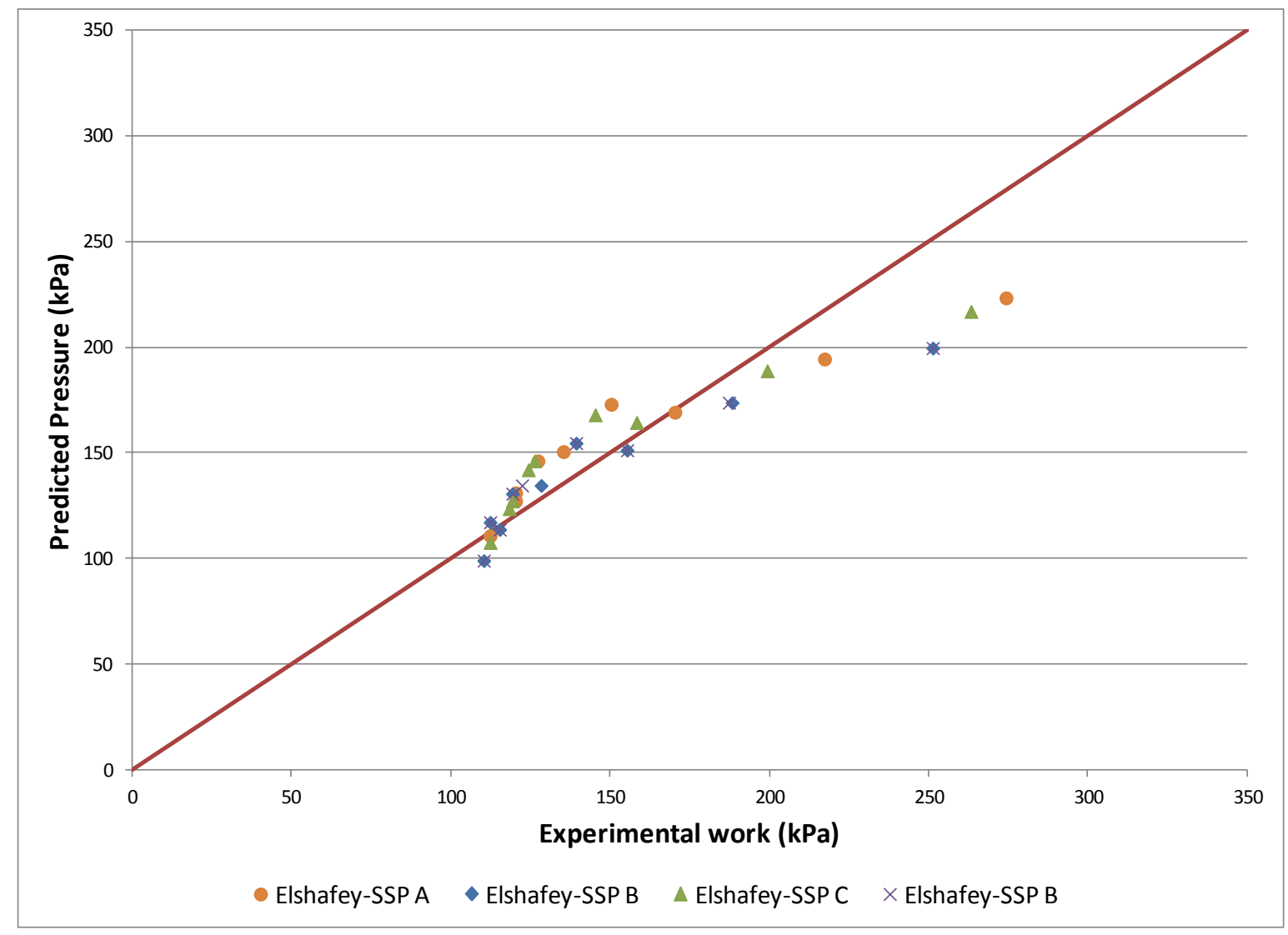

Figure 54: Comparison between experimental pressure values and predicted pressure values 
Figure 54 shows very good agreement between the experimental results and predicted peak incident pressure from the proposed equation. It can be observed from the figure that the performance of both SSP-B and SSP-D were the same. This was expected since they both have the same effective vent area ratio which is the main parameter that affects the performance of VSS section.

The impulse values obtained from Elshafey were also compared to those obtained from the proposed Equation 30. The comparison showed a good correlation between the experimental and predicted results for both SSP-B and SSP-D (Figure 19). However, for the other two configurations, SSP-A and SSP-C (Figure 19), the obtained results from Equation 30 overestimated the experimental results. This is because the ratio of the distance between the two layers to the spacing between the steel angles for the two configurations SSP-A and SSP-C are more than 0.5 . That put these two sections outside the limits where Equation 30 could be valid to calculate the effective vent area ratio of multilayer VSS sections as was concluded in previous section 4.5.3.

Another reason that may have caused this phenomenon is that for the two configurations SSP-A and SSP-C, the vent area ratio was 0.02 which is very small compared that of the other two sections SSP-B and SSP-D which was 0.13 and 0.08 , respectively. So the proposed Equation 30 can be limited to calculate the incident impulse outside VSS containers for sections with effective vent area ratios more than 0.08 . 


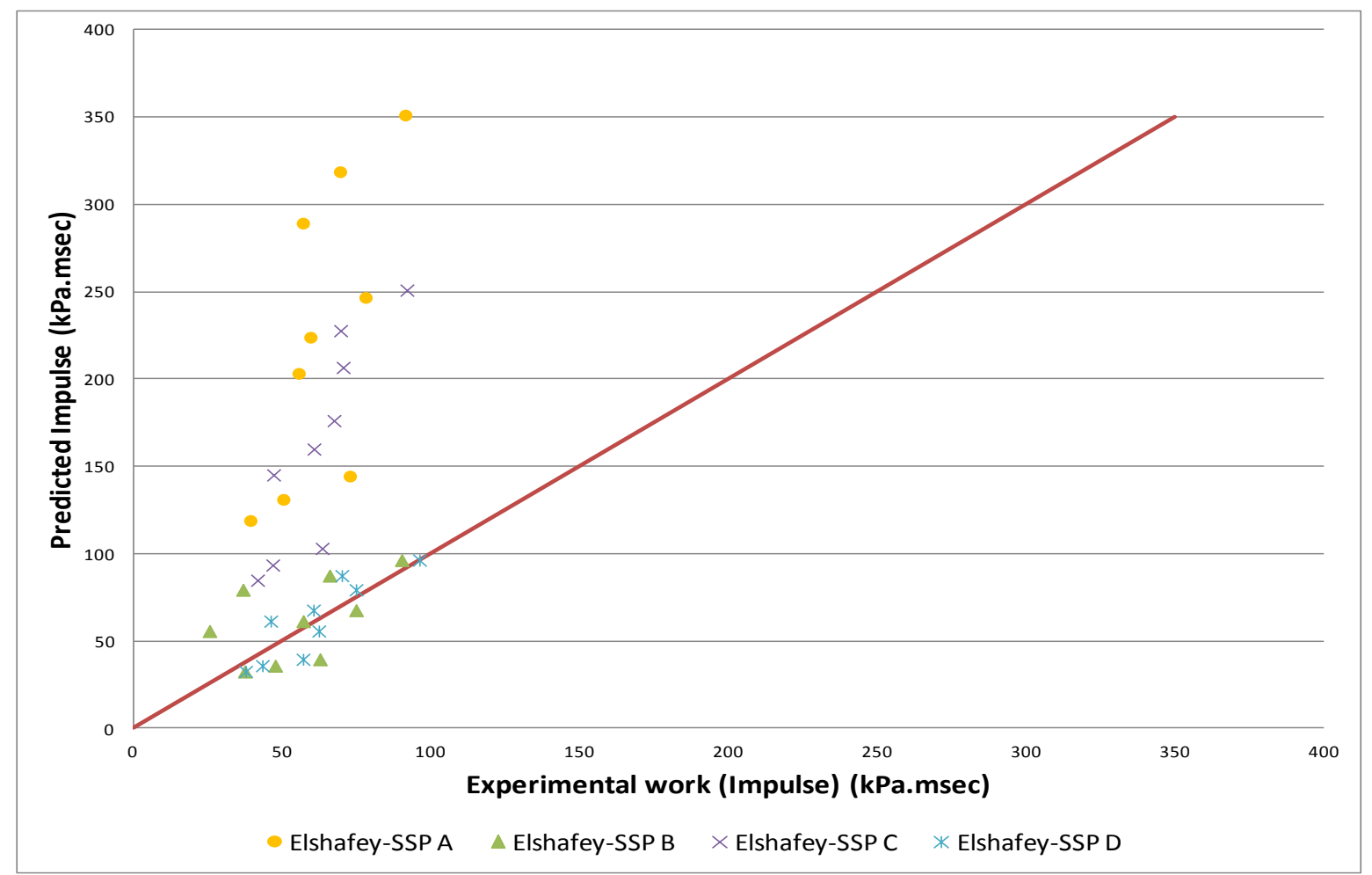

Figure 55: Comparison between experimental Impulse values and predicted Impulse values

\subsection{Summary}

This chapter addressed the performance of different VSS sections. The chapter started by defining the vent area ratio for different single layer VSS sections that were introduced previously by other researchers. Available equations to calculate the effective vent area ratio for multilayer VSS sections was studied and verified using AUTODYN. The level of attenuation of each VSS section was investigated. The optimum vent area ratio that was able to achieve a good level of attenuation was between 0.25 and 0.5 . These two vent area ratios will be included in further investigations in this study.

The level of attenuation of VSS sections was studied at different standoff distances outside the VSS container. The results were analysed and set of empirical equations was introduced to calculate the pressure and impulse outside VSS containers given the charge weight, size of the container and vent area ratio of the VSS section. The obtained results for both pressure and impulse was then compared to previous experimental work and the comparison showed a good correlation between the experimental work results and the calculated pressure and impulse values obtained from the introduced equations. 


\section{Chapter 5: $\quad$ Pressure on Vented Suppressive Shield elements}

\subsection{Introduction}

Pressure profiles on the side walls of vented structures are different from free field reflected pressure profiles. This is due to the reflection of the blast waves on the walls and collision at the center of the container as was discussed earlier in the literature review. The pressure profile on the side wall of a vented structure due to internal explosion differs from one case to another depending on the following parameters:

- Charge weight

- Size of the container

- Vent area ratio

- VSS configuration

In order to obtain a good understanding about the interaction of the blast load with the walls of the container, 2D AUTODYN models were developed with different cases to track the pressure profiles on the VSS elements.

\subsection{Tracking the pressure (2D modeling)}

The pressure on VSS elements was recorded for all the developed models. In AUTODYN, it is not possible to track the pressure on the plate itself since it is modeled as a Lagrange element and is allowed to move. However, it was fixed in the 2D models because the movement of the plates is not required in this model. Figure 56 shows an example for the location of Gauges for the single layer plate configuration with 0.5 vent ratio. 


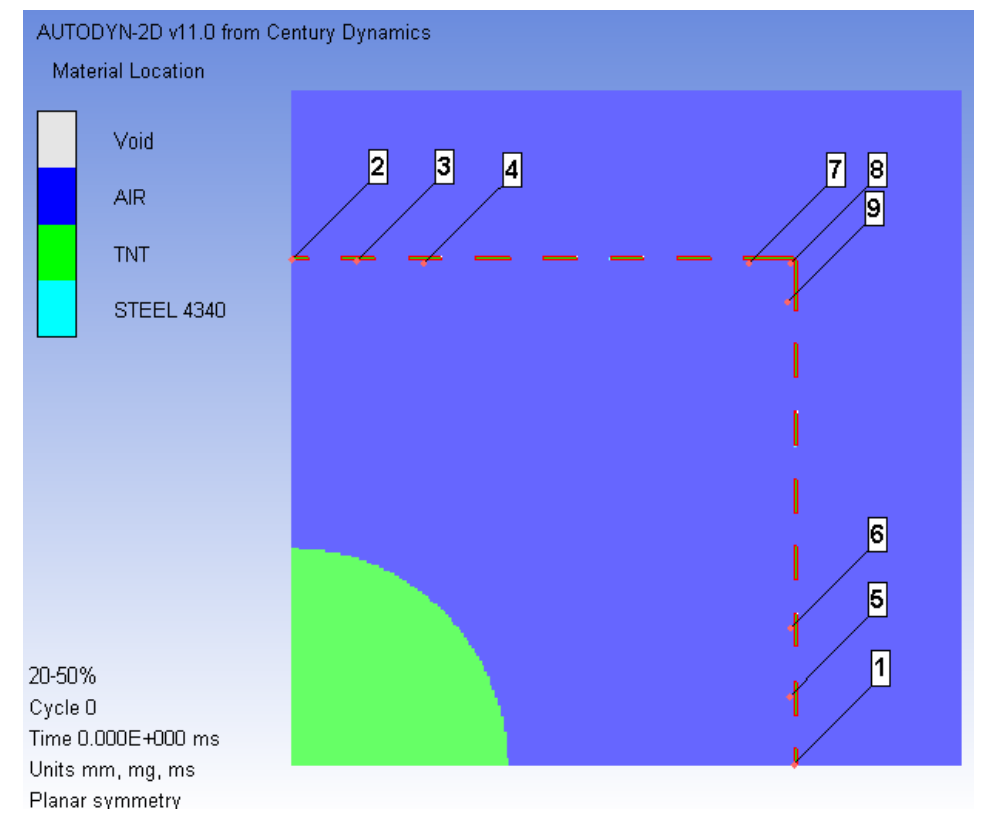

Figure 56: locations of pressure Gauges on plates

The air domain in the model was modeled using Euler mesh that is stationary and no deformation takes place in it. While the plates were modeled using Lagrange mesh where the mesh is allowed to deform and move due to the applied loads. In AUTDOYN, the pressure could only be recorded in the Euler part that is fixed but the pressure that is required to be recorded is at the VSS elements itself which moves during the run of the model. This makes tracking the pressure on the steel angle challenging since the VSS element is moving due to deformation while the Gauges are fixed.

\subsection{Factors affection the pressure profile on plates}

The pressure profile was obtained for the different cases studied. For unvented containers, Baker et al. (1983) proposed a three-peak blast pressure profile to represent loading on the sides of the container (plates). Figure 57 shows the pressure profile obtained from AUTODYN for an unvented $(2.0 \times 2.0 \mathrm{~m})$ container with $20 \mathrm{~kg}$ TNT charge in the center of the container. The location at which this Gauge is located is in the middle of one of the sides of the containers. The figure shows more than one peak pressure on the side wall of the container. These peak pressures are due to the complex reflections inside the unvented container. The peaks highlighted in dotted circles indicate the peak pressures from direct reflection of the wave from the center of detonation to the point of interest. The other peaks are result of other reflections at 
the other side walls of the container. This was indicated by tracking the wave vectors from the AUTODYN output. The ratio of pressures between the first and second peaks was about 2:1 and that between the first to the third peaks was about $4: 1$. These ratios are similar to that proposed by Baker et al. (1983) for unvented containers.

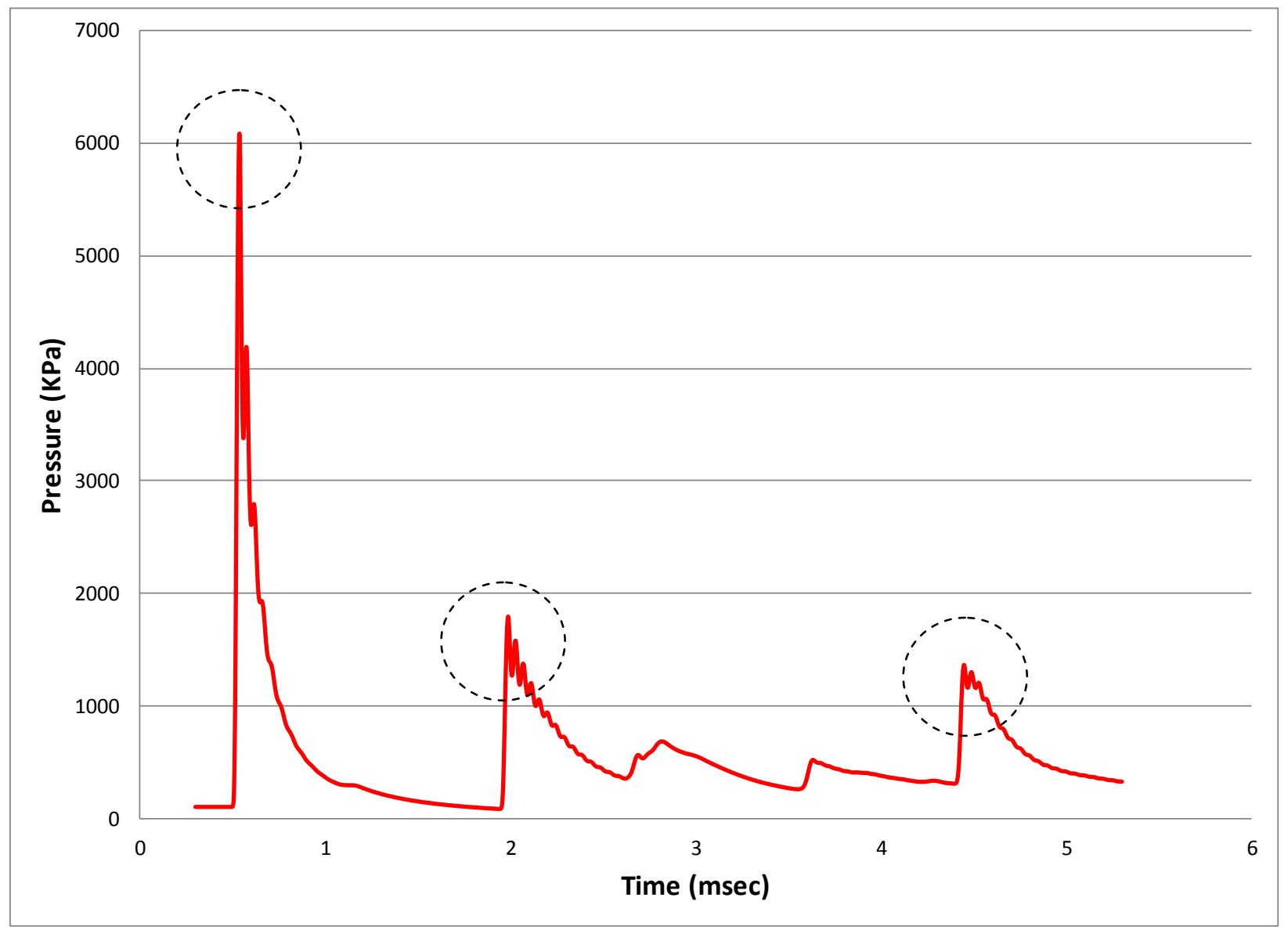

Figure 57: Pressure profile for a confined $(2.0 \times 2.0)$ container

From Figure 57, the gas static pressure remains inside the container because there is no way for it to escape outside the container except when failure of the container occurs and the internal pressure is released. However, when the enclosed structure had vents in its section the pressure profile was changed and the assumption introduced by Baker et al. (1983) doesn't match the actual pressure profile. This is because with the presence of vents within the VSS section, a portion of the blast pressure escapes outside the container and thus affects the pressure profile on the VSS side walls. 


\subsubsection{Effect of vent ratio on the pressure profile}

Vented containers allow gas pressure to escape outside the container. Different 2D AUTODYN models were developed to study the effect of vent ratios on the pressure profile. From chapter 4, it was found that to achieve an effective level of attenuation of blast loads using VSS sections, the vent area ratio should be 0.5 or less. Also, from the literature, it was stated that a structure is considered to be completely opened if the vent area ratio is more than 0.6 (Baker et al., 1983). Therefore, only two vent area ratios were examined in the rest of this study: 0.25 and 0.5. Three different container sizes were considered in the model; $2.0 \times 2.0,3.0 \times 3.0$ and $4.0 \times 4.0$ $\mathrm{m}$. The first peak pressure value is not affected by vent area ratio of the VSS section. Figure 58 shows the difference between the pressure profile for 0.25 and 0.5 vent area ratios with $1 \mathrm{~kg}$ TNT charge inside a $2.0 \times 2.0 \mathrm{~m}$ container compared to an unvented container.

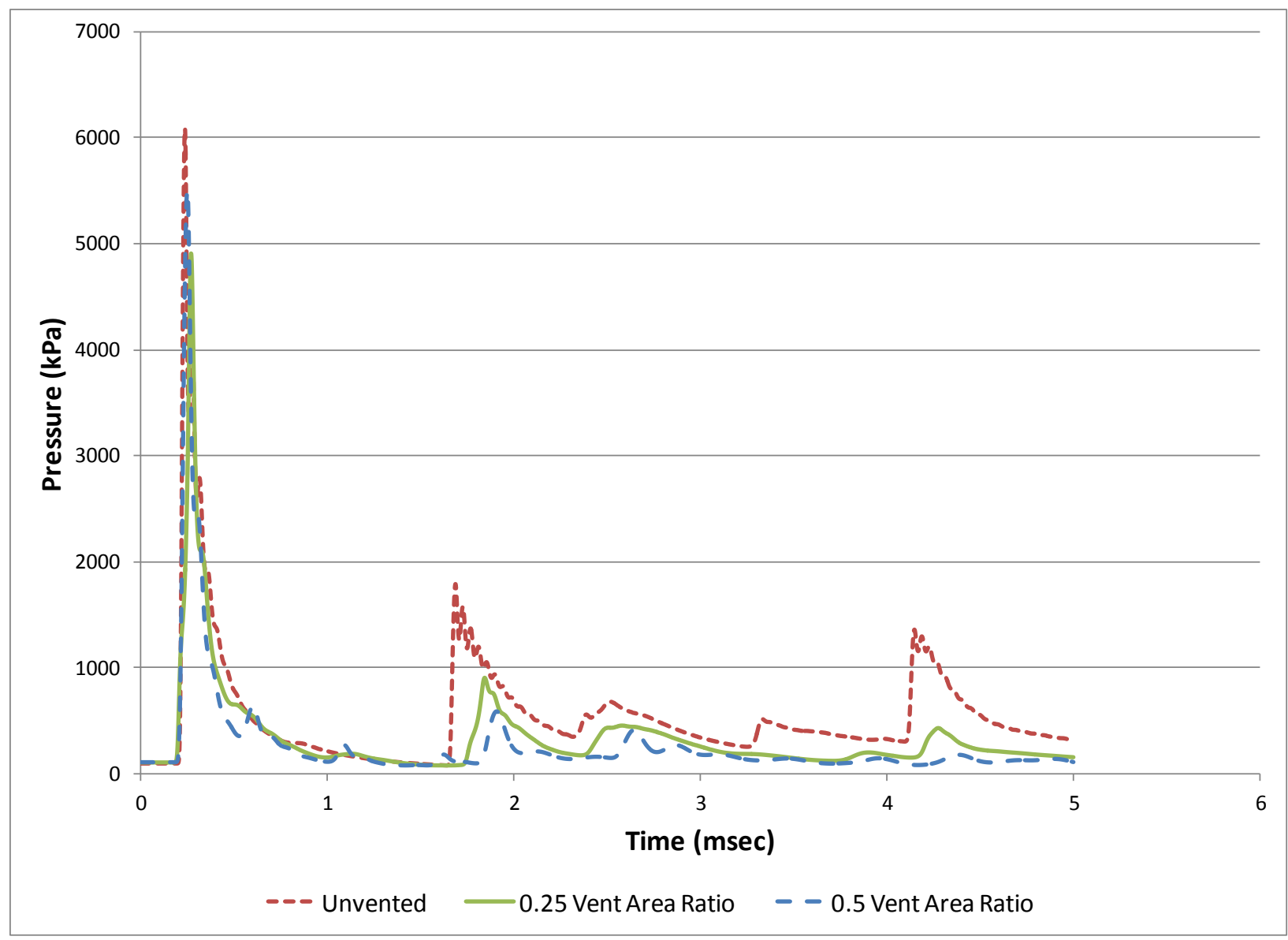

Figure 58: Pressure profile for closed, 0.25 vent area ratio and 0.5 vent area ratio 
From the obtained results, it is observed that the vent ratio affects the pressure profile on the plates. From Figure 58, the effect of the vent ratio can be summarized in the following points:

- The first peak pressure value is constant for the different vent ratios. This is because the distance between the charge and the side wall of the container is the same and the angle of inclination of the plate is the same for the three cases.

- The rate of pressure dissipation is faster for higher vent area ratio. This is due to the reduction of the area subjected to the blast load which makes the blast waves escape faster around the object and hence the rate of dissipation is faster.

- The value of the second peak pressure value is reduced when the vent area ratio increases. This is because the presence of vents in the structure allows more pressure to be released outside the container so the second peak pressure is reduced.

- The time between the first and second peak pressures remains almost the same since the distance required for the blast load to hit the side walls of the container depends mainly on the container size which is constant in this case.

The change in the pressure profile on the side walls of the container reflects on the impulse-time curve. The impulse on the side walls of the container is reduced with the increase of the vent area ratio. Figure 59 shows the impulse value for the same three cases that were addressed to study the pressure profile; unvented structure, vented structure with 0.25 vent area ratio and vented structure with 0.5 vent area ratio. 


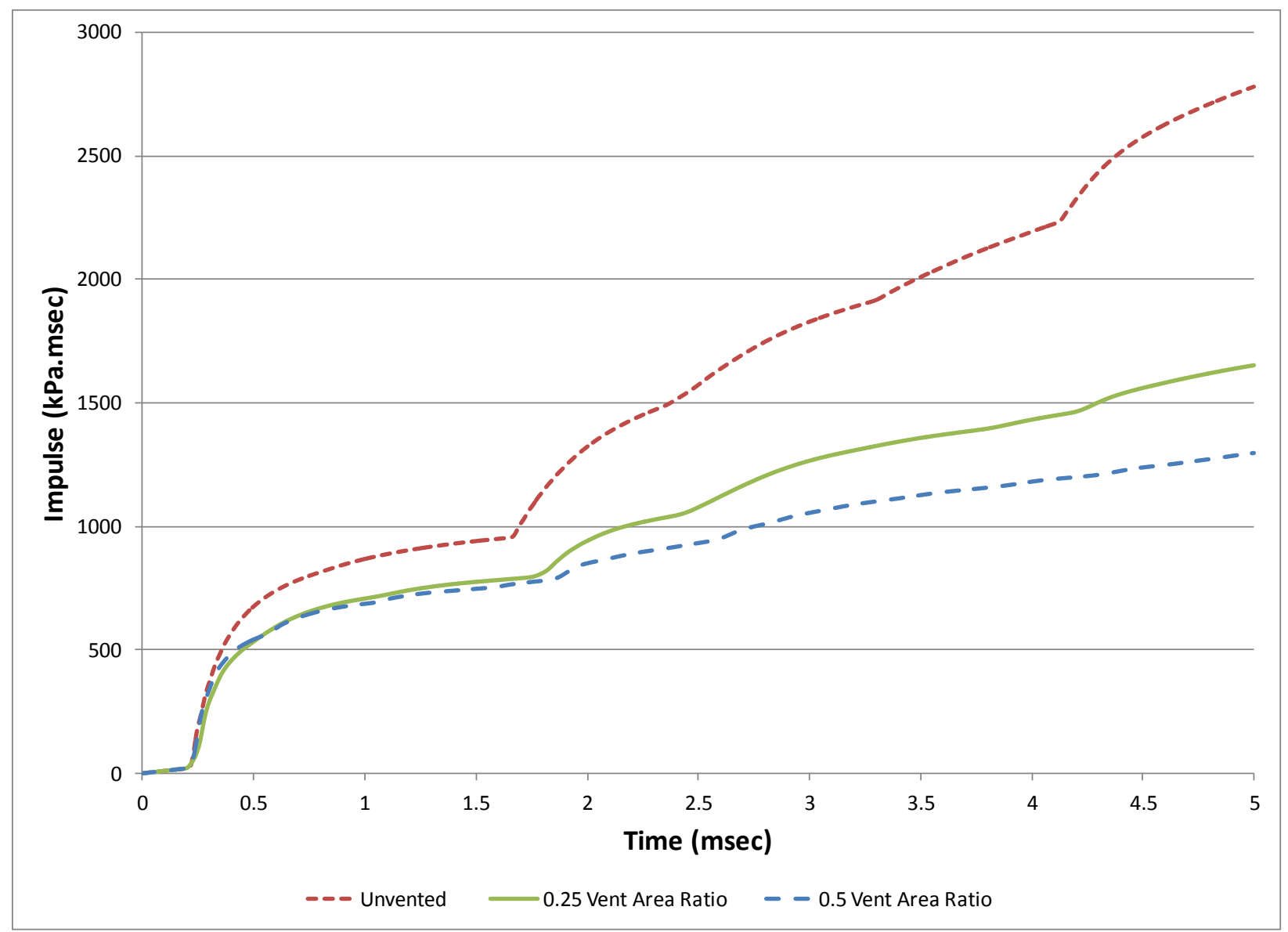

Figure 59: Impulse profile for closed, 0.25 vent area ratio and 0.5 vent area ratio

\subsubsection{Irregular shapes of VSS elements}

In order to enhance the performance of the suppressive shield sections, these plates can be inclined with respect to the direction of the explosion or replaced by angles. The stiffness of angles is higher than those of plates. Consequently, different geometric configurations for VSS sections were modelled in AUTODYN to study the effect of having irregular angles on the performance of the section. Figure 60 shows the geometric configurations that were studied in this research. 


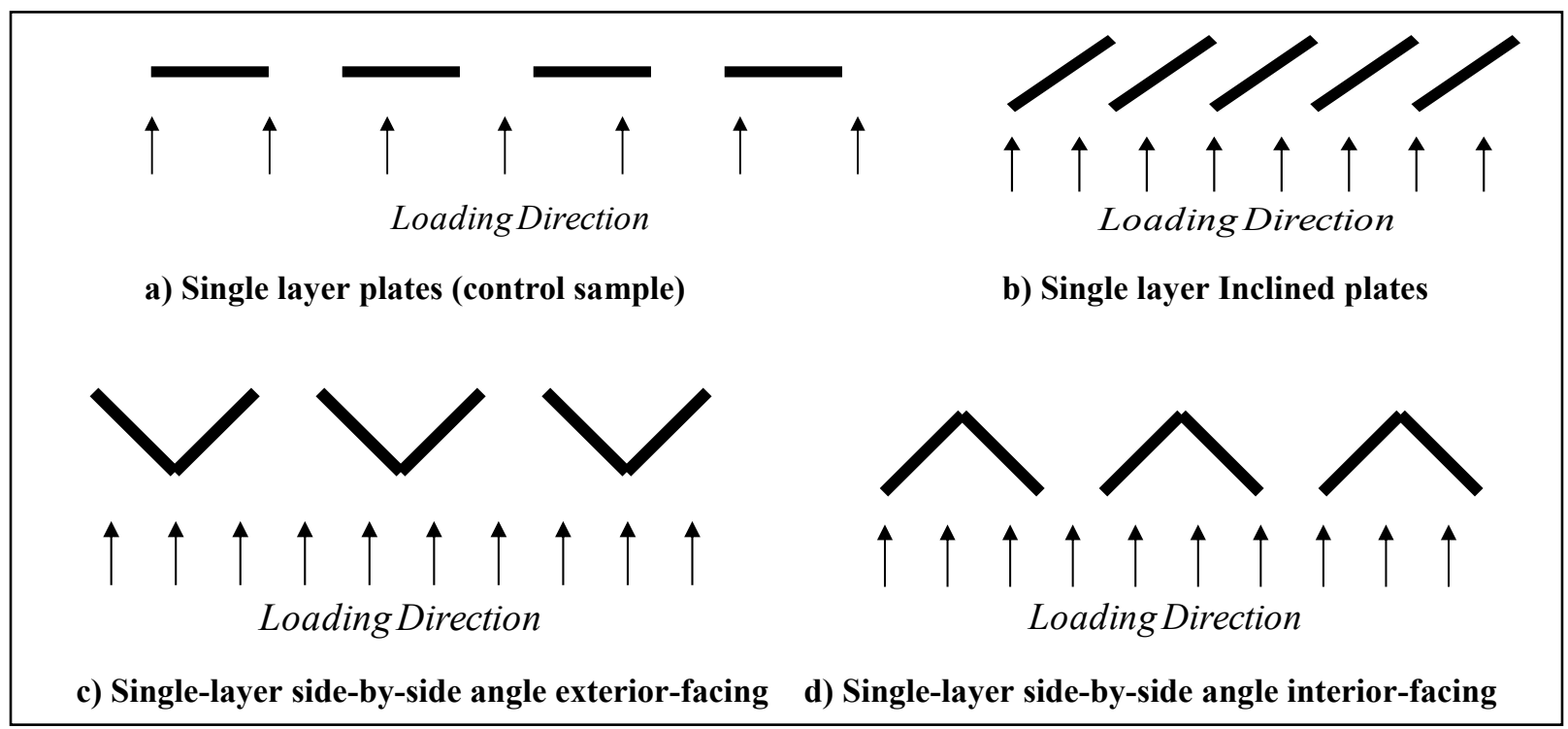

Figure 60: Single layer configurations

In the previous section, the pressure profile on single layer plates was obtained. Figure 61 shows the comparison between different VSS elements; plate, inclined plate and L-shape angle with different positions with respect to the direction of the load. It can be seen from the curve that the reflected pressure at the VSS elements differs as the shape of the angle changes. The side by side interior facing in Figure 60 (d) experienced the highest reflected pressure due to the complex reflections at the internal corners of the angle that increases the reflected pressure. While the side by side exterior facing configuration (Figure 60 (c)) experienced lower pressure than the angles. Although the two angles are of the same size, the orientation of the angle with respect to the direction of the blast load affects the reflected pressure. This will affect the deflection of these angles as will be shown in the next chapter. 


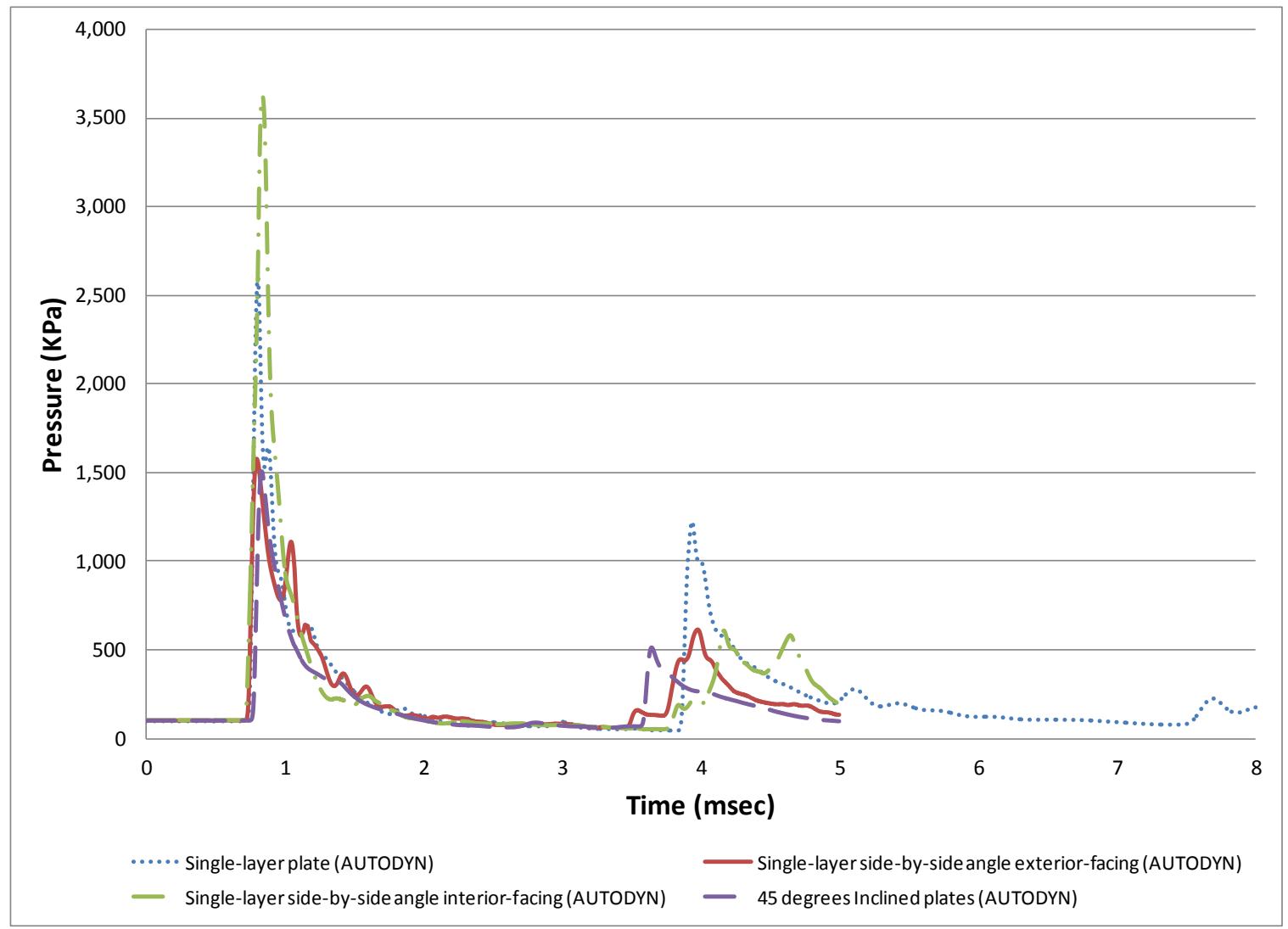

Figure 61: Pressure comparison on VSS elements

It can also be seen from Figure 61 that the pressure on the angles in the side by side exterior facing configuration (Figure 60 (c)) has roughly the same pressure profile of the inclined plates (Figure 60 (b)). This is because the maximum pressure on the angle in case of the side by side exterior facing (Figure 60 (c)) is on the side of the angle which has similar conditions of the inclined plates in terms of angle of inclination of the plates with respect to the blast load.

Another observation that was found from Figure 61 is that the second peak pressure is lower in the case of inclined plates. This is due to the geometry of the plate that makes the reflected waves propagate in other directions and not back to the center of detonation. As a result, the reflected waves at the center of detonation are less than the reflected waves in case of plates that are perpendicular to the blast wave direction. This also affects the impulse on the angles. The value of the impulse at the angles will differ depending on the duration that the pressure lasts on the angle. Figure 62 shows the impulse on the angles for different configurations. 


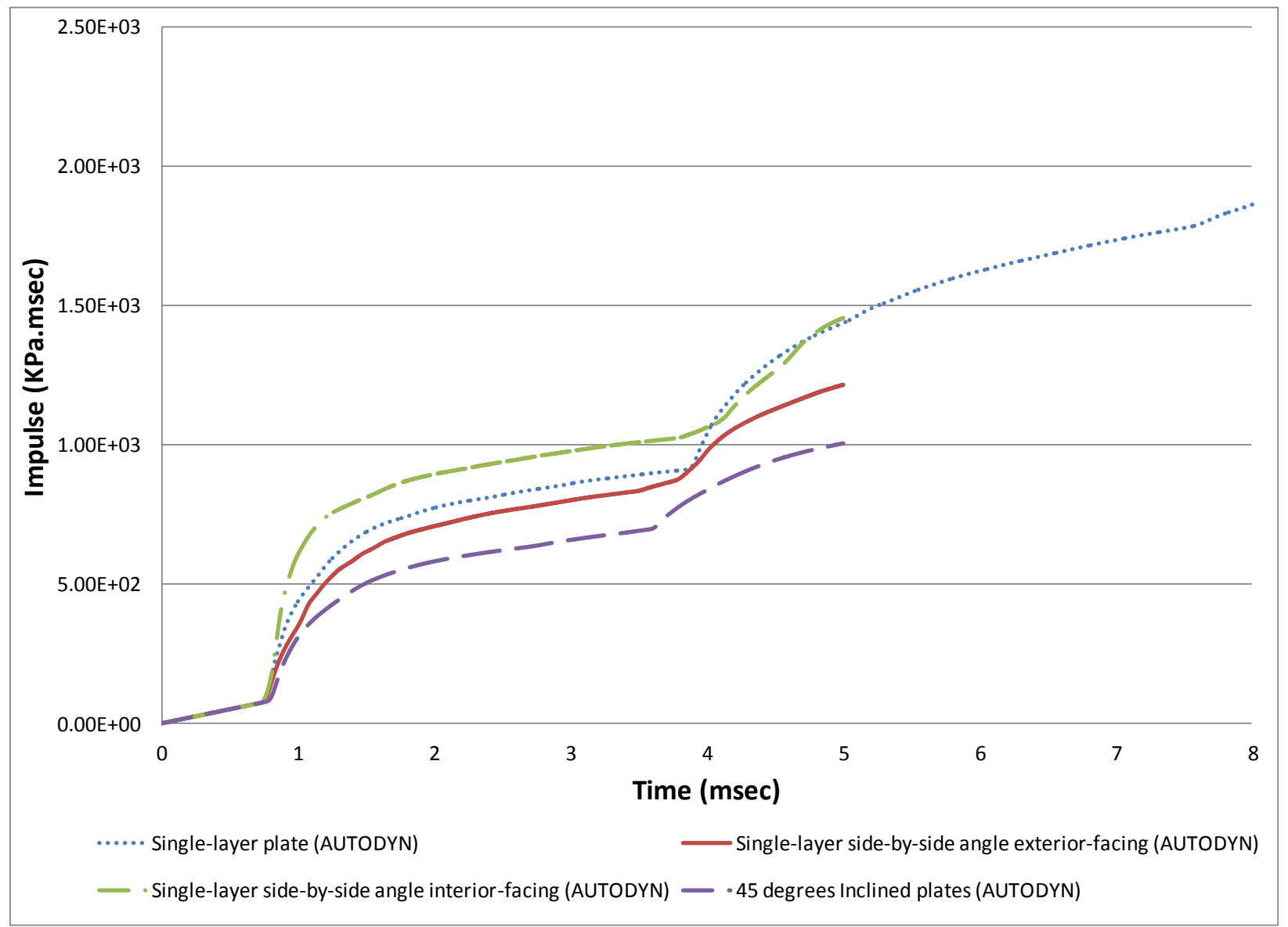

Figure 62: Impulse comparison on VSS elements

From Figure 62, it can be seen that the single layer side-by-side interior facing configuration experienced the highest impulse value. This is again because of the geometry of the angle in this configuration. While for the inclined plates configuration experienced the least impulse value. This is because blast wave reflection in the side-by-side exterior facing has capacity to get back into the container while for the inclined plate the pressure is allowed to escape easily and no further reflections occur in this zone.

It should be noted that although the reflected pressure for the side by side exterior facing configuration and that of the inclined plate configuration are the same, the impulse value is different. This may be explained that the inclined plate configuration allows the waves to escape smoothly through the shields. On the other hand the side by side exterior facing configuration, some reflections will take place on the angle due to the presence of the other angle that affects the escaping of the blast waves. 


\subsubsection{Geometric coefficient}

As shown in the previous results, the reflected pressure values on angles are different depending on the geometric configuration of the VSS section. The pressure and Impulse profiles were recorded for different TNT charges on different angles. The ratio between the peak pressures and impulses for irregular VSS sections to single layer plate configuration was calculated. This ratio i reflect the effect of the geometric configuration on the reflected pressure and impulse on VSS elements. These values are known as geometric coefficients. The single layer plate configuration was used as the control configuration to which other configurations were compared to. Three configurations were addressed in this part. The three studied configurations are the inclined plate configuration, Single-layer side-by-side angle (interiorfacing) and Single-layer side-by-side angle (exterior-facing). Table 8 and Table 9 show the ratio between the reflected pressure and Impulse, respectively, on VSS elements for different geometric configurations and the plate configuration (control configuration).

Table 8: Geometric coefficient for different VSS configurations (for pressure on angles)

\begin{tabular}{|c|c|c|c|c|}
\hline \multicolumn{5}{|c|}{ Comparison between geometric coefficients (Pressure on angles) } \\
\hline $\begin{array}{l}\text { TNT } \\
\text { charge }\end{array}$ & $\begin{array}{c}\text { Container } \\
\text { size }\end{array}$ & $\begin{array}{l}\text { Inclined plate } \\
\text { configuration }\end{array}$ & $\begin{array}{l}\text { Side by side (exterior } \\
\text { facing) configuration }\end{array}$ & $\begin{array}{l}\text { Side by side (interior } \\
\text { facing) configuration }\end{array}$ \\
\hline 1 & $2.0 \times 2.0$ & 0.5 & 0.5 & 2.1 \\
\hline 5 & $2.0 \times 2.0$ & 0.5 & 0.5 & 1.6 \\
\hline 10 & $2.0 \times 2.0$ & 0.5 & 0.5 & 1.2 \\
\hline 20 & $2.0 \times 2.0$ & 0.4 & 0.7 & 2 \\
\hline 1 & $3.0 \times 3.0$ & 0.6 & 0.4 & 1.4 \\
\hline 5 & $3.0 \times 3.0$ & 0.5 & 0.4 & 1.9 \\
\hline 10 & $3.0 \times 3.0$ & 0.4 & 0.4 & 1.9 \\
\hline 20 & $3.0 \times 3.0$ & 0.4 & 0.6 & 1.9 \\
\hline 1 & $4.0 \times 4.0$ & 0.6 & 0.6 & 1.5 \\
\hline 5 & $4.0 \times 4.0$ & 0.5 & 0.5 & 1.6 \\
\hline 10 & $4.0 \times 4.0$ & 0.6 & 0.4 & 1.6 \\
\hline 20 & $4.0 \times 4.0$ & 0.5 & 0.5 & 1.5 \\
\hline
\end{tabular}


Table 9: Geometric coefficient for different VSS configurations (for Impulse on angles)

\begin{tabular}{|c|c|c|c|c|}
\hline \multicolumn{5}{|c|}{ Comparison between geometric coefficients (Impulse on angles) } \\
\hline $\begin{array}{c}\text { TNT } \\
\text { charge }\end{array}$ & $\begin{array}{c}\text { Container } \\
\text { size }\end{array}$ & $\begin{array}{c}\text { Inclined plate } \\
\text { configuration }\end{array}$ & $\begin{array}{c}\text { Side by side (exterior facing) } \\
\text { configuration }\end{array}$ & $\begin{array}{c}\text { Side by side (interior facing) } \\
\text { configuration }\end{array}$ \\
\hline 1 & $2.0 \times 2.0$ & 0.5 & 0.9 & 1.25 \\
\hline 5 & $2.0 \times 2.0$ & 0.6 & 0.9 & 1 \\
\hline 10 & $2.0 \times 2.0$ & 0.5 & 1 & 1.2 \\
\hline 20 & $2.0 \times 2.0$ & 0.5 & 0.7 & 1.15 \\
\hline \hline 1 & $3.0 \times 3.0$ & 0.45 & 0.9 & 1 \\
\hline 5 & $3.0 \times 3.0$ & 0.3 & 0.75 & 1.23 \\
\hline 10 & $3.0 \times 3.0$ & 0.35 & 0.8 & 1.2 \\
\hline 20 & $3.0 \times 3.0$ & 0.4 & 0.7 & 0.9 \\
\hline \hline 1 & $4.0 \times 4.0$ & 0.3 & 0.9 & 1.3 \\
\hline 5 & $4.0 \times 4.0$ & 0.4 & 0.8 & 1.25 \\
\hline 10 & $4.0 \times 4.0$ & 0.5 & 0.9 & \\
\hline 20 & $4.0 \times 4.0$ & 0.6 & 0.9 & \\
\hline
\end{tabular}

The summary of the two tables are presented in Table 10. It should be noted that the geometric coefficients to calculate the reflected pressure values are different that those used to calculate the reflected impulse.

Table 10: Geometric coefficient for different VSS configurations

\begin{tabular}{|c|c|c|c|c|}
\hline $\begin{array}{c}\text { Geometric } \\
\text { coefficient }\end{array}$ & $\begin{array}{c}\text { Single } \\
\text { plate } \\
\text { (control) }\end{array}$ & $\begin{array}{c}\text { Inclined plate } \\
\text { configuration }\end{array}$ & $\begin{array}{c}\text { Side by side (exterior } \\
\text { facing) configuration }\end{array}$ & $\begin{array}{c}\text { Side by side (interior } \\
\text { facing) configuration }\end{array}$ \\
\hline $\begin{array}{c}\text { Pressure } \\
\text { on VSS } \\
\text { angles }\end{array}$ & 1 & 0.5 & 0.5 & 1.7 \\
\hline $\begin{array}{c}\text { Impulse on } \\
\text { VSS } \\
\text { angles }\end{array}$ & 1 & 0.45 & 0.85 & 1.14 \\
\hline
\end{tabular}

The calculated geometric coefficients were based on the first reflected peak pressure and Impulse. In order to investigate the effect of these factors on the entire pressure profile, the actual pressure profile on the VSS elements obtained from AUTODYN was compared to the pressure profile of a single layer plate multiplied by the developed geometric coefficient depending on the geometric configuration used. This will be done for the four VSS elements that were mentioned 
earlier. Figure 63, Figure 64 and Figure 65 show a comparison between the pressure profile obtained from AUTODYN and those obtained from using the geometric coefficients.

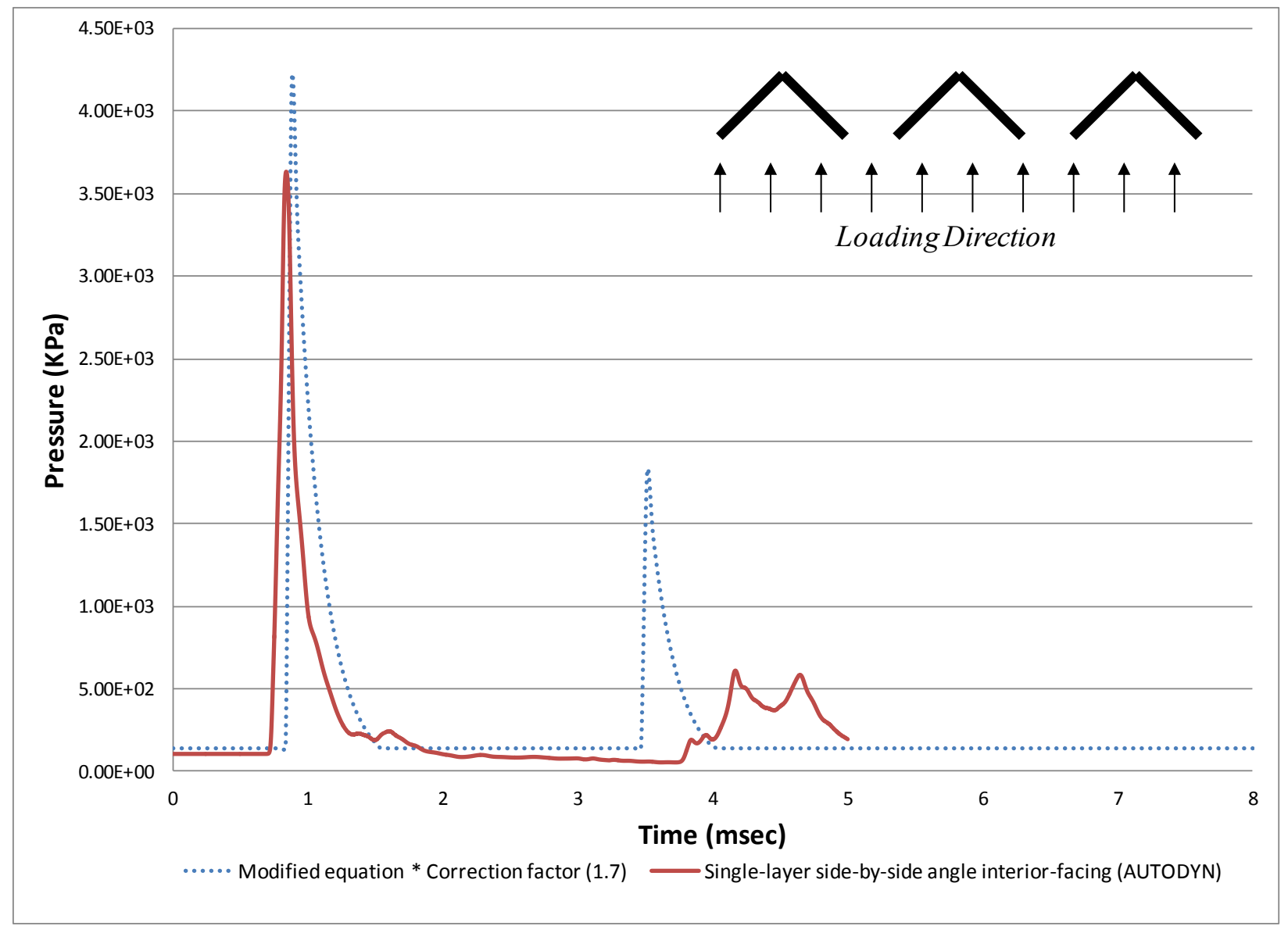

Figure 63: Comparison between the actual pressure profile from AUTODYN and the predicted pressure using the geometric coefficient for Single-layer side-by-side angle interior-facing 


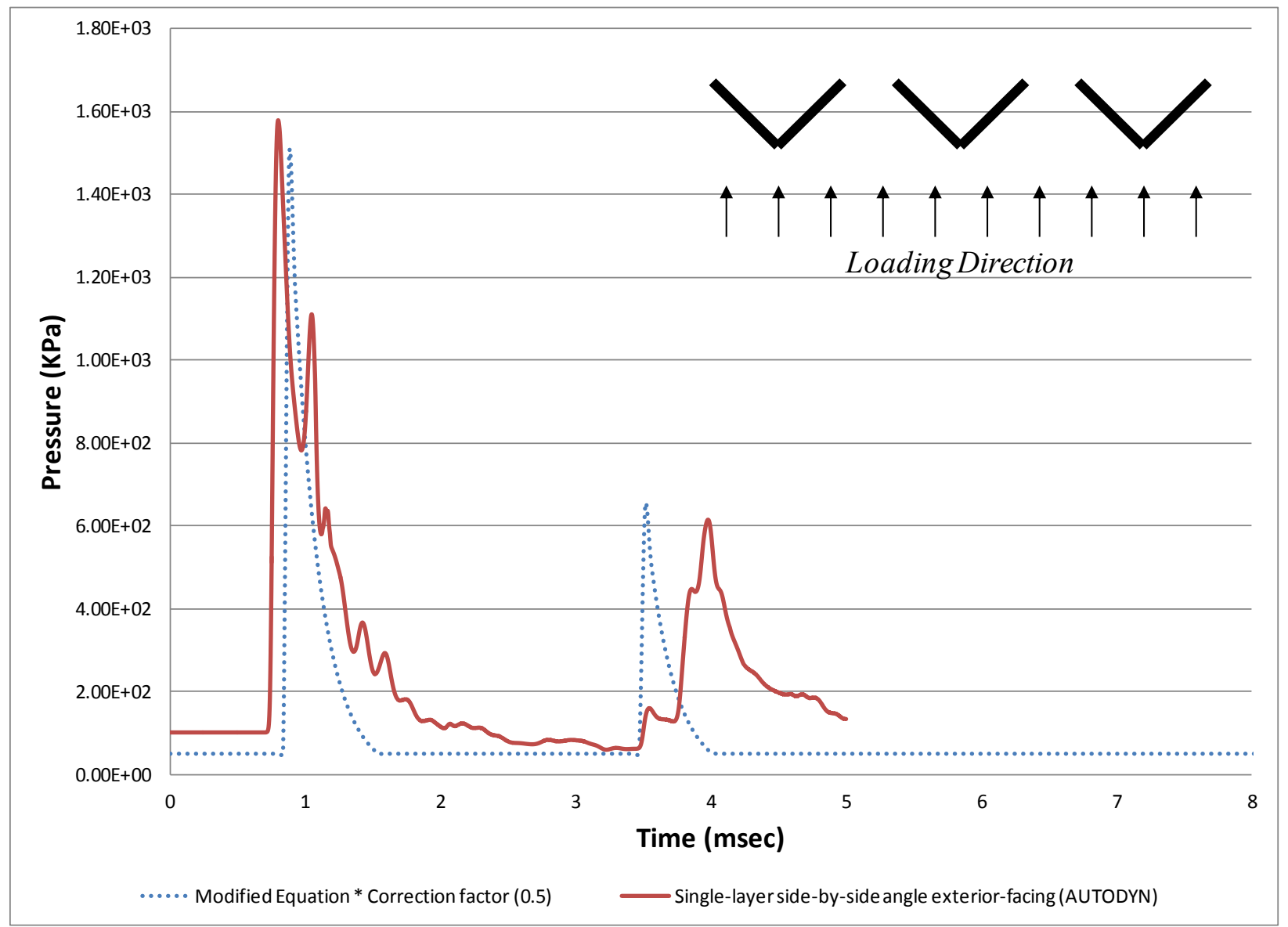

Figure 64: Comparison between the actual pressure profile from AUTODYN and the predicted pressure using the geometric coefficient for Single-layer side-by-side angle exterior-facing 


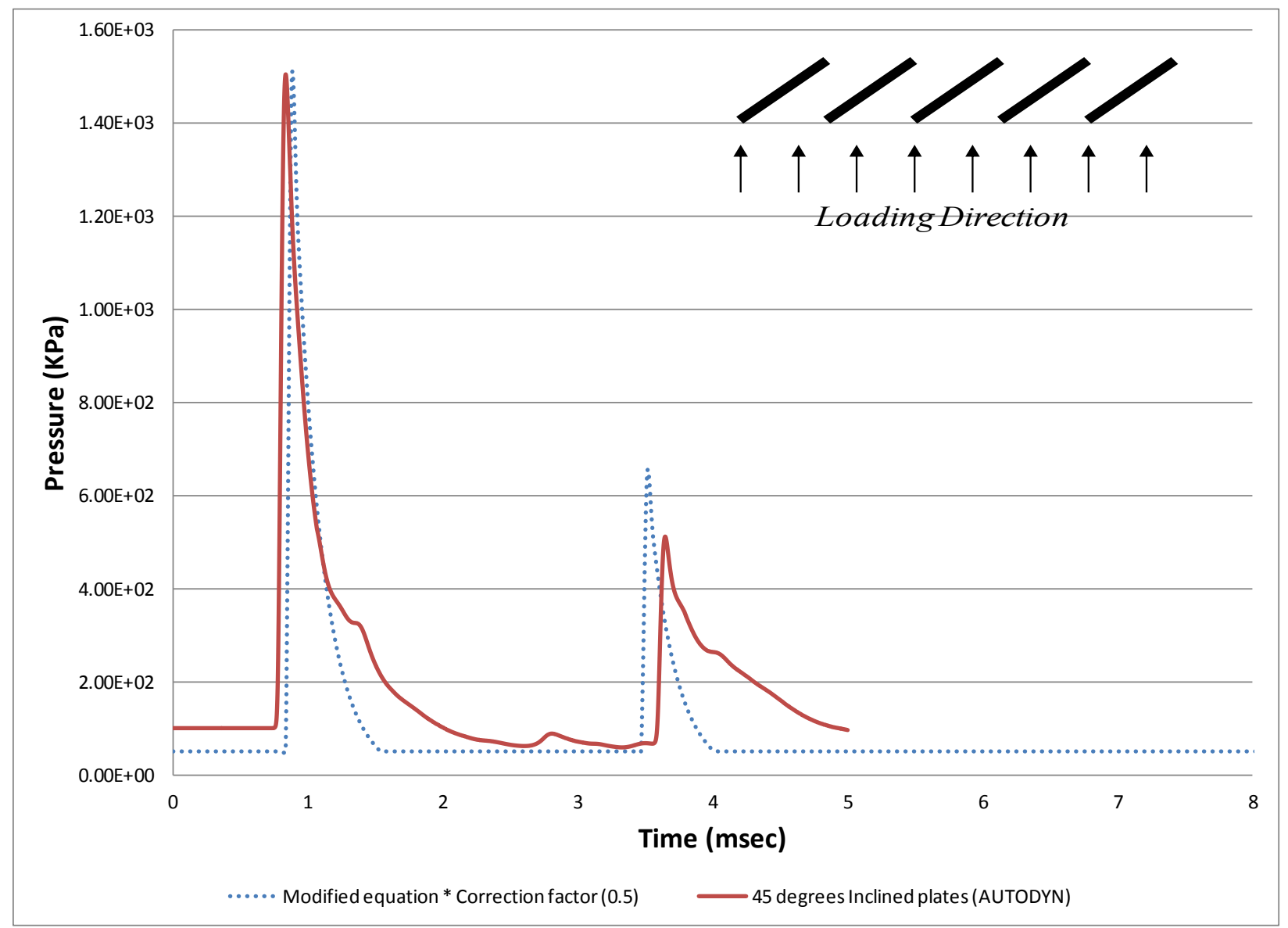

Figure 65: Comparison between the actual pressure profile from AUTODYN and the predicted pressure using the geometric coefficient for Single-layer inclined plates

\subsubsection{Multilayer Vented Suppressive shield}

As mentioned before, one of the disadvantages of single layer vented suppressive shield sections is that there is a direct path for the blast wave to exit outside the container. Using a second layer of suppressive shield staggered with the first prevents having these direct paths. The performance of the multilayer VSS sections on attenuation of peak pressure outside VSS containers was studied in chapter 4. In this section, the pressure profile on the angles of the multilayer system will be addressed. Two multilayer VSS sections, presented in Figure 66 were studied in this thesis. 

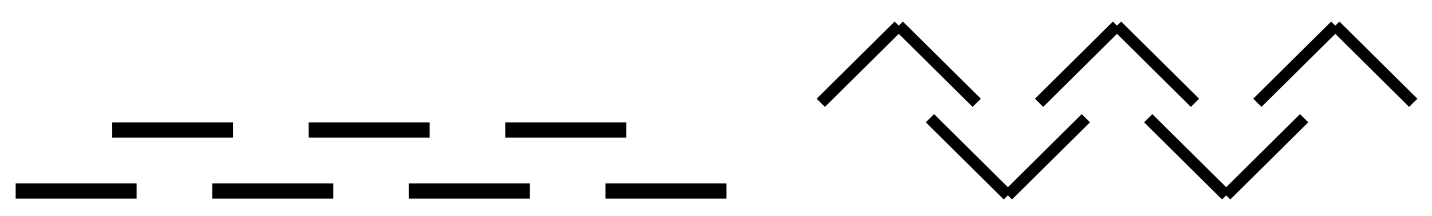

Figure 66: (a) Double-layer plate configuration, (b) Double-layer nested side-by-side angle configuration (Koger et al., 1975)

\subsubsection{Doubled layer plate}

The pressure profile on each layer of the multilayer VSS section differs depending on the geometric configuration of the VSS section. Figure 67 shows a comparison of the pressure profile on the plates of the doubled layer plate section. The comparison is between the inside and outside row of plates. Also, the single VSS section with the same vent area ratio is included in the comparison to show the pressure difference between the single and the multilayer VSS plate section.

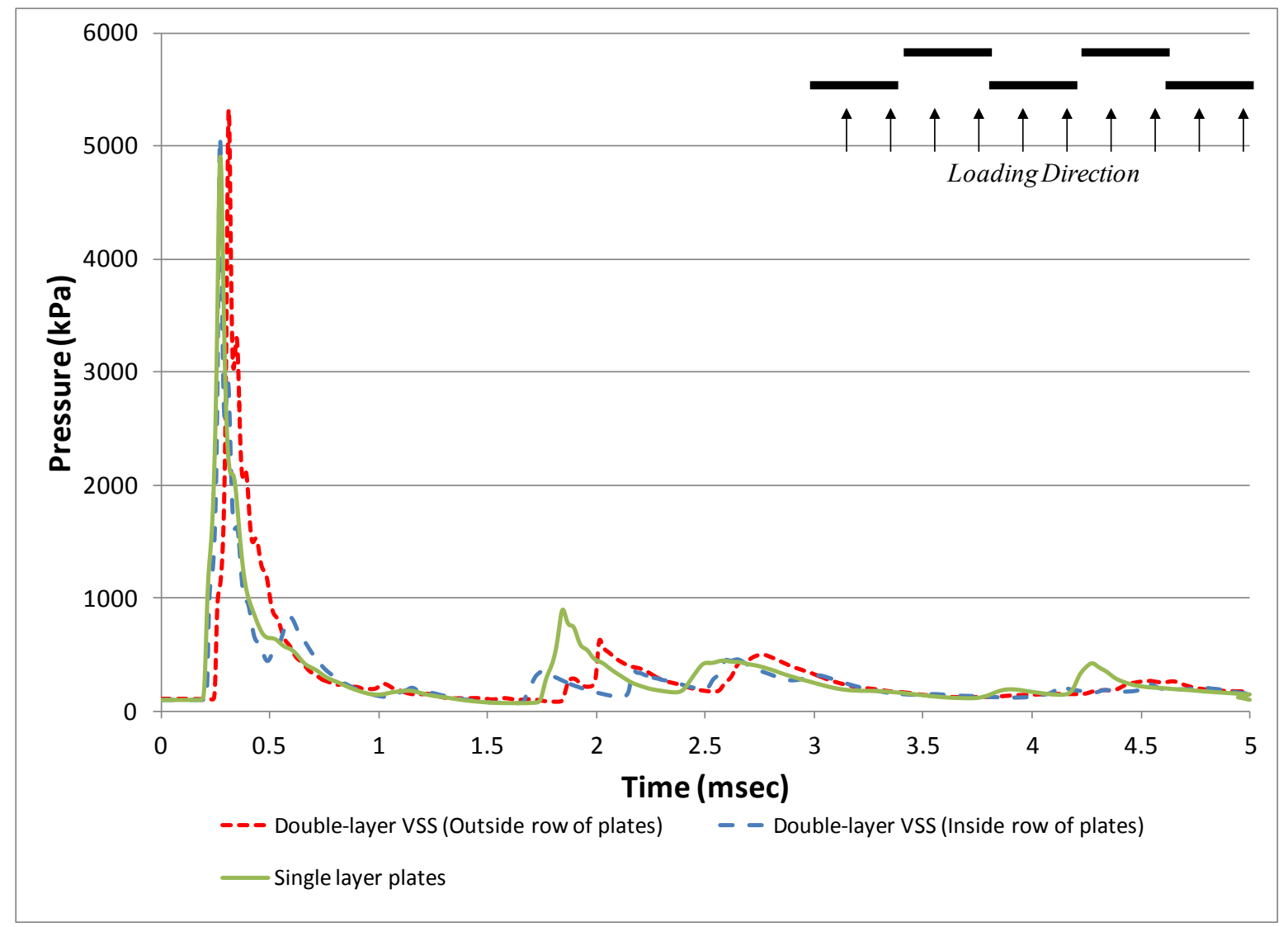

Figure 67: Pressure profile on plates of a doubled VSS plate section 
From Figure 67, it is observed that the pressure profile on the two plates is almost the same. However, the reflected pressure on the outside row of plates is slightly higher than the inside row. This can be explained by the presence of blast jets from the interaction of the blast wave with the inside row of plates. These jets increase the velocity of the blast wave; therefore the outside row of plates experienced higher pressure than the inside one. The velocity vectors of the blast wave are shown in Figure 68. The figure shows the jets that are experienced on the outside row of plates due to the geometry of the section.

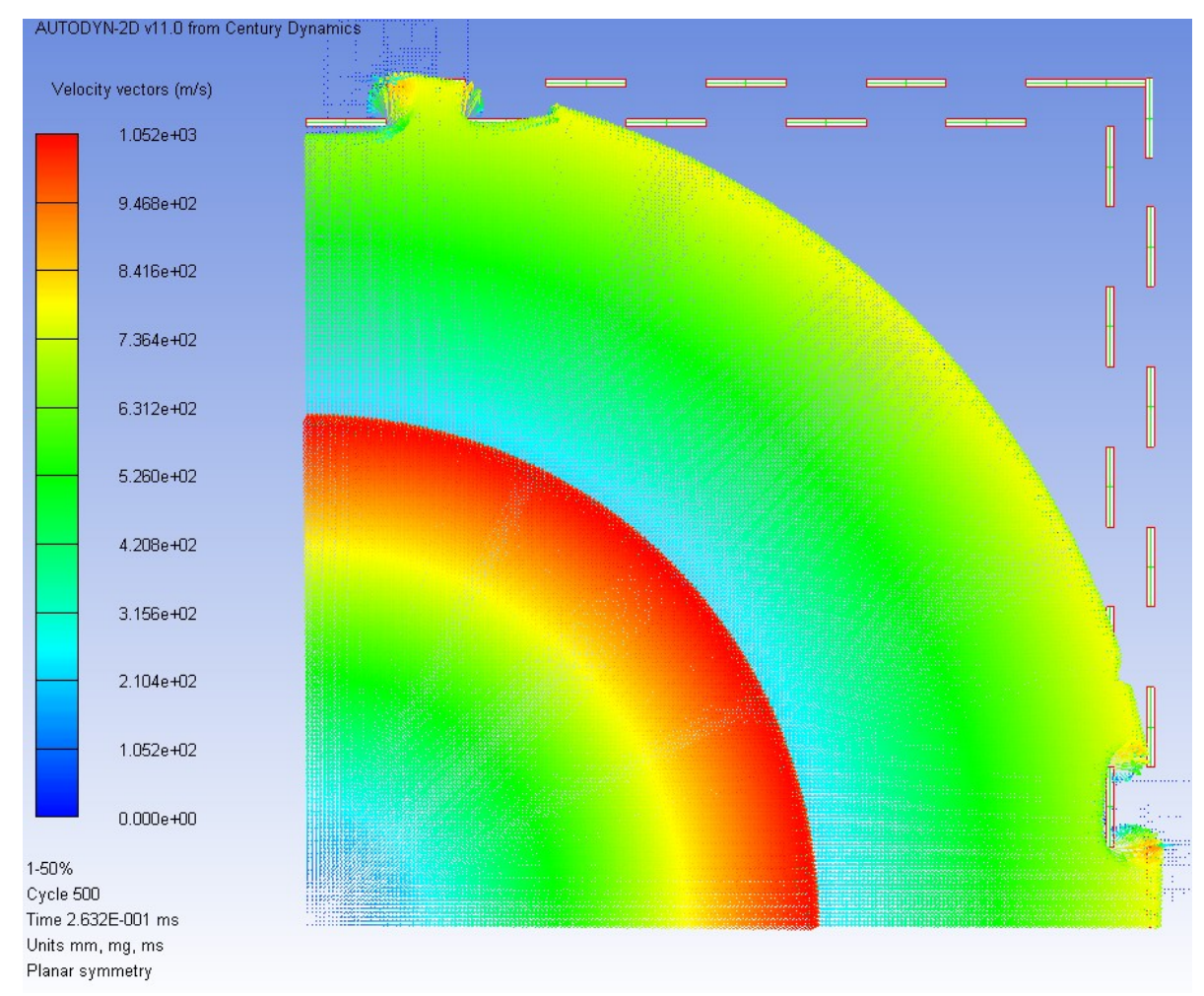

Figure 68: Velocity vectors showing the jets that occurs in the double layer VSS

Another observation is that the second reflected peak pressure is higher in case of the single layer plate. This is explained that although the two sections have the same vent area ratio, the reflecting surface is different that affects the direction of the reflected waves from the VSS side wall. In case of the double layer plate configuration, the directions of the reflected waves are not all with the same direction as in case of the single layer plate configuration due to the reflection of these waves the two layers of plates in the configuration. This affects the intensity of the reflected waves and thus affecting the second reflected peak pressure. 


\subsubsection{Double-layer nested side-by-side angles}

The double layer section consists of two layers of nested side-by side that are arranged in a staggered manner (as shown in Figure 66 (b)) in order to prevent having direct paths of blast waves. The pressure profile was recorded at the angles for both rows (inside and outside). Figure 69 shows the pressure profile on the angles in both rows as well as the pressure profile for both the single layer side by side angle (exterior and interior facing).

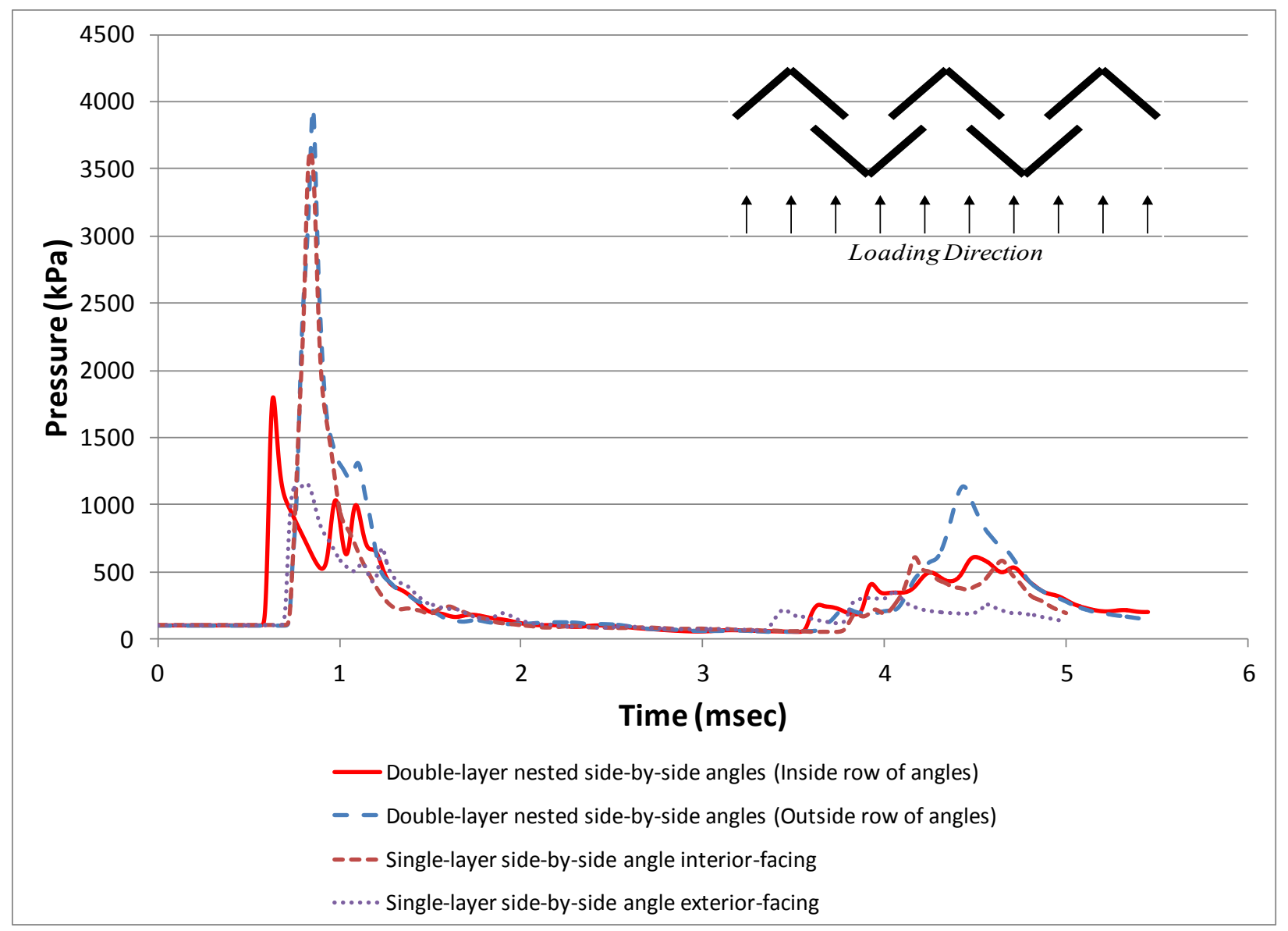

Figure 69: Pressure profile on angles of Double-layer nested side-by-side angles

From Figure 69, it can be seen that the pressure profile mainly depends on the geometry of the angle assemble. So the reflected pressure at the vertex of the angle in the outside row is equal to that of the single layer side by side angle (interior facing). However, a slight increase in the first peak pressure was observed in case of the double layer system and again, that is because of the effect of jets from the inside row of angles as discussed before in the case of the double layer plate configuration. 


\subsection{Empirical equations to calculate the pressure on the side walls of vented Structures}

In the earlier sections of this chapter, the reflected pressure on VSS elements was studied and a good understanding of the interaction between the blast waves and different VSS sections was achieved. However, all the results were obtained using AUTODYN software package that requires a long computation time as well as specialized expertise. The results from AUTODYN are analysed in this section in order to achieve an empirical method for calculating the reflected pressure on the side wall elements of VSS sections without using CFD programs such as AUTODYN.

The results for all the modelled cases were analysed and a set of equations was introduced to predict the pressure on the side walls elements of vented structures. The simplest form of a blast wave has been described by the Friedlander waveform (Kingery, 1984), so this equation was used as a reference. The Friedlander waveform equation is shown in Equation 31 and it is used to calculate the pressure time profile of blast wave. The parameters such as $P_{s o}, t_{d}$ and other related parameters are required to determine the pressure profile. These parameters are calculated using another set of equations that are presented in Appendix B. After obtaining those parameters, the pressure profile is calculated by the Equation 31 (Lam et al., 2004). The factor, $b$, is the parameter controlling the decay rate of the wave amplitude and is usually calculated using trial and error method depending on the impulse value.

$$
P(t)=\text { Coefficient } \times P_{r}\left(1-\frac{t}{t_{d}}\right) e^{\frac{-b}{t_{d}}}
$$

Equation 31

Where $P_{r}$ is the maximum reflected pressure at the point of interest. The "Coefficient" in Equation 31 equals to 1.0 for spherical blast, and equals to 1.8 for hemispherical blast (Lam et al., 2004). The coefficient $b$ is the parameter controlling the rate of wave amplitude decay and $t_{d}$ is the duration of the blast pulse. In most cases, the value of $b$ varied between 1.5 and 5 (Lam et al., 2004).

\subsubsection{Unvented container}

Figure 70 shows the comparison between the pressure profiles obtained from AUTODYN for an unvented structure and that obtained from Equation 31. It is observed from 
the figure that the Friedlander waveform equation was able to predict the peak reflected pressure; however the equation didn't count for the consecutive reflected peaks. Also, the figure shows that the rate of decay obtained from the equations was different than that of the curve obtained from AUTODYN. This was expected since these equations were only developed to calculate the pressure in the case of free field explosion. Therefore, some modifications to the equation were required in order to account for the reflected pressures on the walls of the container due to internal explosion as well as the decay rate of the blast load.

The Pressure profile obtained from AUTODYN showed a good correlation with the assumption made by Baker (1983). The curve shows that for unvented structures, three peaks should be taken into account. The ratio between the first to second peak was $2: 1$ and the ratio between the first to the third was 4:1. This coincides with what was introduced by Baker (1983).

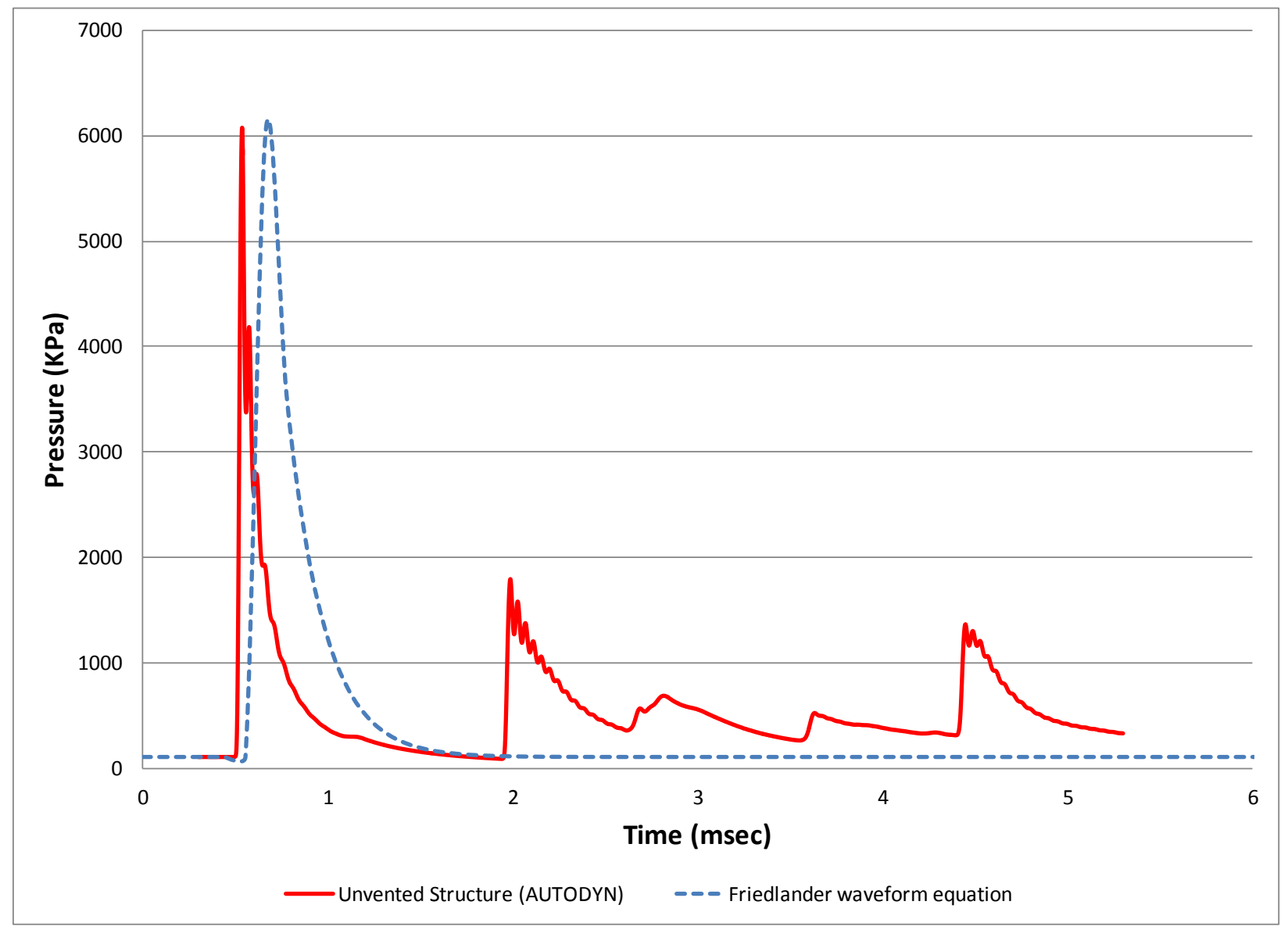

Figure 70: Comparison between the available proposed equation and the actual pressure 


\subsubsection{Prediction of pressure inside Vented structures}

Three modifications need to be made to the current Friedlander equation in order to predict the pressure on the side walls of vented structures. The first modification is re-evaluating the decay coefficient, $b$, to be able to predict the pressure profile in case of internal explosion. The second modification is to take into account the consecutive peak pressures and to study the ratio between the peak pressures. The third parameter that needs to be studied is the time between the pressure peaks. The three parameters are studied and explained in details in this section taking into account the effect of different charge masses, container sizes and vent area ratios.

\subsubsection{The decay parameter}

The decay parameter, $b$, changes with the change of the size of the object facing the blast load. In case of vented structures, the area facing the blast load contains openings which reduce the area of the solid part which the blast load encounters. As the vent area increases, the size of the solid part of the side wall of the structure decreases and therefore the rate of decaying increases. Also the value of $b$ affects the value of impulse which is computed as the area under the pressure time curve. The load on the plate's decays much faster in case of internal explosion than free field explosion due to the geometry of the structure or in other words, the value $b$ depends on the vent ratio and the size of the container. In the equation, the term that affects the rate of decay is the decay parameter $b$.

The AUTODYN results showed that the value $b$ presented by Lam et al (2004) is valid in case of internal explosion but within specific limits. This equation is valid when the value of $\mathrm{Z}$ varies between 0 and 0.68 . For $Z$ values greater than 0.68 , the value $b$ is assumed to be 10 . The rate of dissipation is assumed to be the same for both peak pressures.

\subsubsection{The ratio between the first to the second peak pressure}

The ratio between consecutive blast peaks on the VSS side wall depends on the vent area ratio of the VSS section. From the previous results, it was found that the effective vent area ratios that were able to attenuate the pressure inside were the 0.25 and 0.5 . Therefore, these two 
vent area ratios were studied in more details. Table 11 shows the pressure value for the first and second peak for the studied cases.

Table 11: Ratio between first and second reflected pressures

\begin{tabular}{|c|c|c|c|c|c|}
\hline $\begin{array}{c}\mathrm{W} \\
(\mathrm{Kg})\end{array}$ & $\begin{array}{c}\text { Container } \\
\text { Size }(\mathrm{mxm})\end{array}$ & $\begin{array}{l}\text { Vent } \\
\text { Ratio }\end{array}$ & $\begin{array}{l}\text { First Reflected } \\
\text { pressure }(\mathrm{kPa})\end{array}$ & $\begin{array}{c}\text { Second } \\
\text { Reflected } \\
\text { pressure }(\mathrm{kPa})\end{array}$ & $\begin{array}{c}\text { Factor } \\
\text { (1st/2nd) }\end{array}$ \\
\hline \multirow{6}{*}{1} & $2.0 \times 2.0$ & 0.25 & 5,079 & 1,100 & 4.6 \\
\hline & $2.0 \times 2.0$ & 0.5 & 5,018 & 850 & 5.9 \\
\hline & $3.0 \times 3.0$ & 0.25 & 2,590 & 800 & 3.2 \\
\hline & $3.0 \times 3.0$ & 0.5 & 2,680 & 700 & 3.8 \\
\hline & $4.0 \times 4.0$ & 0.25 & 1,501 & 480 & 3.1 \\
\hline & $4.0 \times 4.0$ & 0.5 & 1,630 & 300 & 5.4 \\
\hline \multirow{6}{*}{5} & $2.0 \times 2.0$ & 0.25 & 14,820 & 5,000 & 3.0 \\
\hline & $2.0 \times 2.0$ & 0.5 & 15,230 & 4,000 & 3.8 \\
\hline & $3.0 \times 3.0$ & 0.25 & 9,671 & 2,150 & 4.5 \\
\hline & $3.0 \times 3.0$ & 0.5 & 9,500 & 1,800 & 5.3 \\
\hline & $4.0 \times 4.0$ & 0.25 & 6,000 & 1,500 & 4.0 \\
\hline & $4.0 \times 4.0$ & 0.5 & 6,000 & 980 & 6.1 \\
\hline \multirow{6}{*}{10} & $2.0 \times 2.0$ & 0.25 & 34,690 & 11,000 & 3.2 \\
\hline & $2.0 \times 2.0$ & 0.5 & 35,770 & 8,600 & 4.2 \\
\hline & $3.0 \times 3.0$ & 0.25 & 16,330 & 5,400 & 3.0 \\
\hline & $3.0 \times 3.0$ & 0.5 & 16,000 & 3,200 & 5.0 \\
\hline & $4.0 \times 4.0$ & 0.25 & 11,000 & 3,000 & 3.7 \\
\hline & $4.0 \times 4.0$ & 0.5 & 10,820 & 2,000 & 5.4 \\
\hline \multirow{6}{*}{20} & $2.0 \times 2.0$ & 0.25 & 58,330 & 18,690 & 3.1 \\
\hline & $2.0 \times 2.0$ & 0.5 & 56,500 & 15,780 & 3.6 \\
\hline & $3.0 \times 3.0$ & 0.25 & 33,780 & 14,600 & 2.3 \\
\hline & $3.0 \times 3.0$ & 0.5 & 33,220 & 9,550 & 3.5 \\
\hline & $4.0 \times 4.0$ & 0.25 & 25,170 & 9,480 & 2.7 \\
\hline & $4.0 \times 4.0$ & 0.5 & 24,730 & 6,730 & 3.7 \\
\hline
\end{tabular}

The results showed that ratio between the first and second peak pressure depends on the area vent ratio. A regression analysis was carried out on the results mentioned in Table 11. The ratio between the first to the second is $4: 1$ for vented structure with 0.25 vent area ratio. While for the vented structures with 0.5 vent area ratio, the ratio between the first to the second is $6: 1$. It 
was also found that the pressure peaks beyond this point could be neglected because they are very small to affect the pressure on the side wall of the VSS containers.

\subsubsection{Time between the two peaks}

The timing between the first and the second peak pressure represents the time for the shock wave required to propagate from the side wall of the structure to the center of detonation and back to the side wall of the container. So it is expected that this parameter depends on the container size. The time required for the blast wave to propagate from the center of detonation to the side wall of the container is known as the arrival time $\left(t_{a}\right)$. Assuming that the blast wave propagates with the same speed, the second peak pressure is expected to be at $\left(3 t_{a}\right)$ or less due to the compressing of the air median where the shock wave is propagating through. However, from the results, it was found that the timing between the peak pressure waves is longer than this time for most studied cases. This could be explained that the reflected shock wave that is propagating from the structure walls back to the center of detonation experience resistance due to the other waves coming from the other direction (center of detonation towards the container side walls). This resistance reduce the speed of the waves and thus increasing the time. Another reason that affects the speed of the wave propagating is the loss of energy of the shock wave after striking the side wall of the container and reflecting back inside. This can be more explained in Figure 71 shows the timing at which the shock wave strikes the side wall of the VSS containers. 


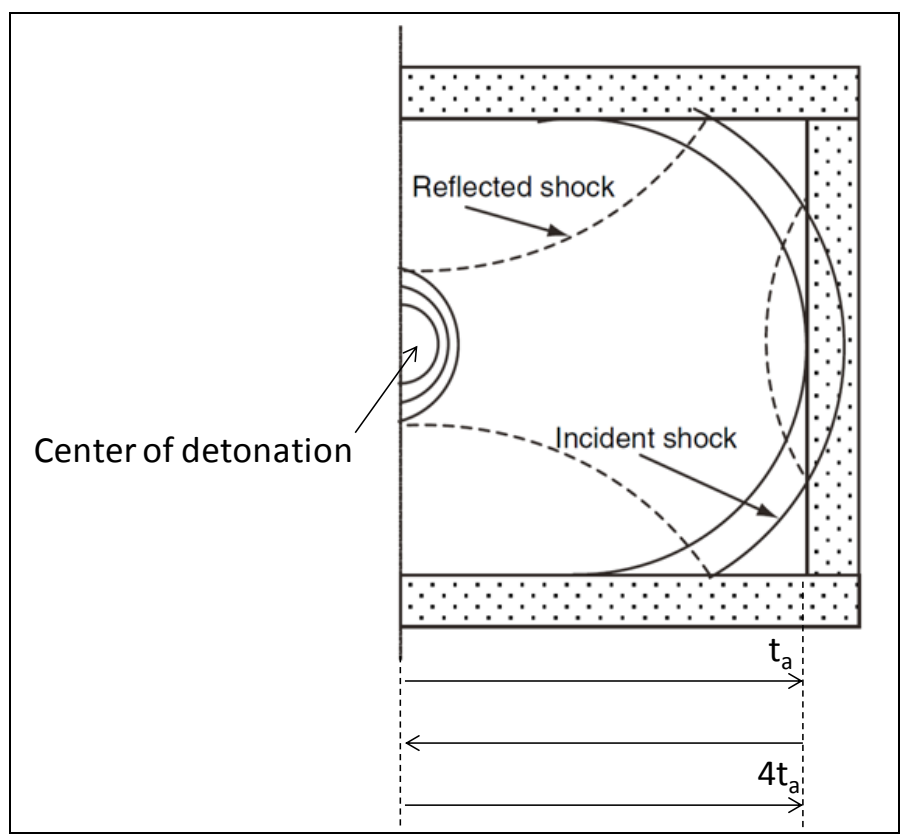

Figure 71: Arrival time at the side wall of the container

\subsubsection{Proposed equation to predict the pressure on side walls of vented structures}

After applying the three modifications to the original equation, the proposed set of equations is shown below:

$$
\begin{array}{ll}
\text { If } \quad t<t_{a} & P_{r}(t)=0 \\
\text { If } \quad t_{a}<t<t_{a}+t_{d} & P_{r}(t)=\text { Coefficient } * P_{r}\left(1-\frac{t}{t_{d}}\right) e^{\frac{-b}{t_{d}}} \\
\text { If } \quad 4 \mathrm{t}_{\mathrm{a}}<\mathrm{t} & P_{r}(t)=\text { Coefficien } \mathrm{t} * \frac{P_{r}}{V_{\text {factor }}}\left(1-\frac{t}{t_{d}}\right) e^{\frac{-b}{t_{d}}}
\end{array}
$$

Where $V_{\text {factor }}$ relates to the relation between the first and second peak pressure values, $b$ is the decay parameter and given as:

$$
\begin{array}{ll}
b=Z^{2}-3.7 Z+4.2 & \text { for } 0<Z<0.68 \\
b=10 & \text { for } Z>0.68
\end{array}
$$


For the second peak pressure value, it depends on the vent ratio of the container. Figure 58 presented earlier shows the pressure time profile for three cases of $2.0 \times 2.0 \mathrm{~m}$ suppressive shields subjected to $20 \mathrm{~kg}$ of TNT charge in the center of the container. The three cases were unvented container, vented container with 0.25 vent area ratio, and vented container with 0.5 vent area ratio. From the figure, it was observed that the ratio between the first and second peak pressure is affected by the vent ratio in the container. Following is the predicted ratios between the two peak pressures according to the vent area ratio of the vented structure.

$$
\begin{array}{ll}
\frac{P_{1}}{P_{2}}=2 & \text { For vent area ratio }=0 \text { (Unvented }) \\
\frac{P_{1}}{P_{2}}=4 & \text { For vent area ratio }=0.25 \\
\frac{P_{1}}{P_{2}}=6 & \text { For vent area ratio }=0.5
\end{array}
$$

\subsubsection{Comparison between AUTODYN results and proposed equations}

Figure 72 shows the prediction of the set of modified equations to match the pressure profile obtained from AUTODYN models. Figure 72 shows the pressure profile comparison between AUTODYN and proposed equations for $1 \mathrm{~kg}$ TNT in a $(3.0 \times 3.0 \mathrm{~m})$ VSS container with an area vent ratio equals to 0.5 . 


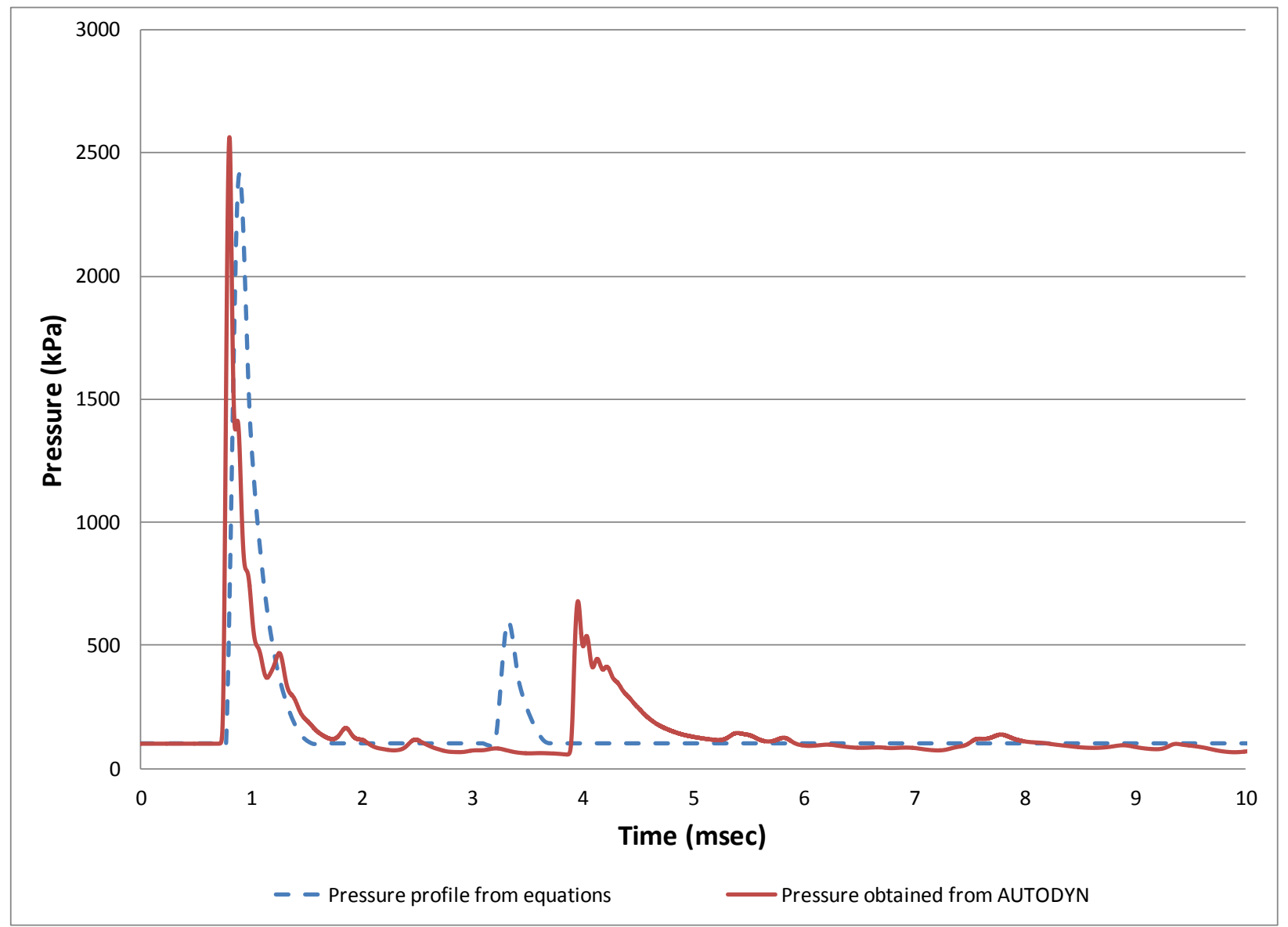

Figure 72: Pressure profile comparison between AUTODYN and proposed equations for $1 \mathrm{~kg}$ TNT in a $(3.0 \times 3.0 \mathrm{~m})$ container with vent ratio equals 0.25

Figure 72 shows a good prediction to the pressure profile on the angles. More results for different explosive charges and container sizes are presented in Appendix C. This obtained pressure profile form the equations will be used later on the design of the angles of the suppressive shield section.

\subsubsection{Prediction of Impulse}

The pressure profile affects the impulse value on the plates. The impulse value is very important for the design of the structural element. The impulse value reflects the time where the pressure is applied on the plates. Impulse is computed as the area under the pressure-time profile. The area under the curve was calculated by dividing the area under the curve into trapezoids. The under the curve is divided into time steps. Figure 73 shows the comparison between the Impulse 
from AUTODYN and the impulse from the approximate computation of the area under the curve. The curve shows a reasonable prediction of the impulse profile on the plates.

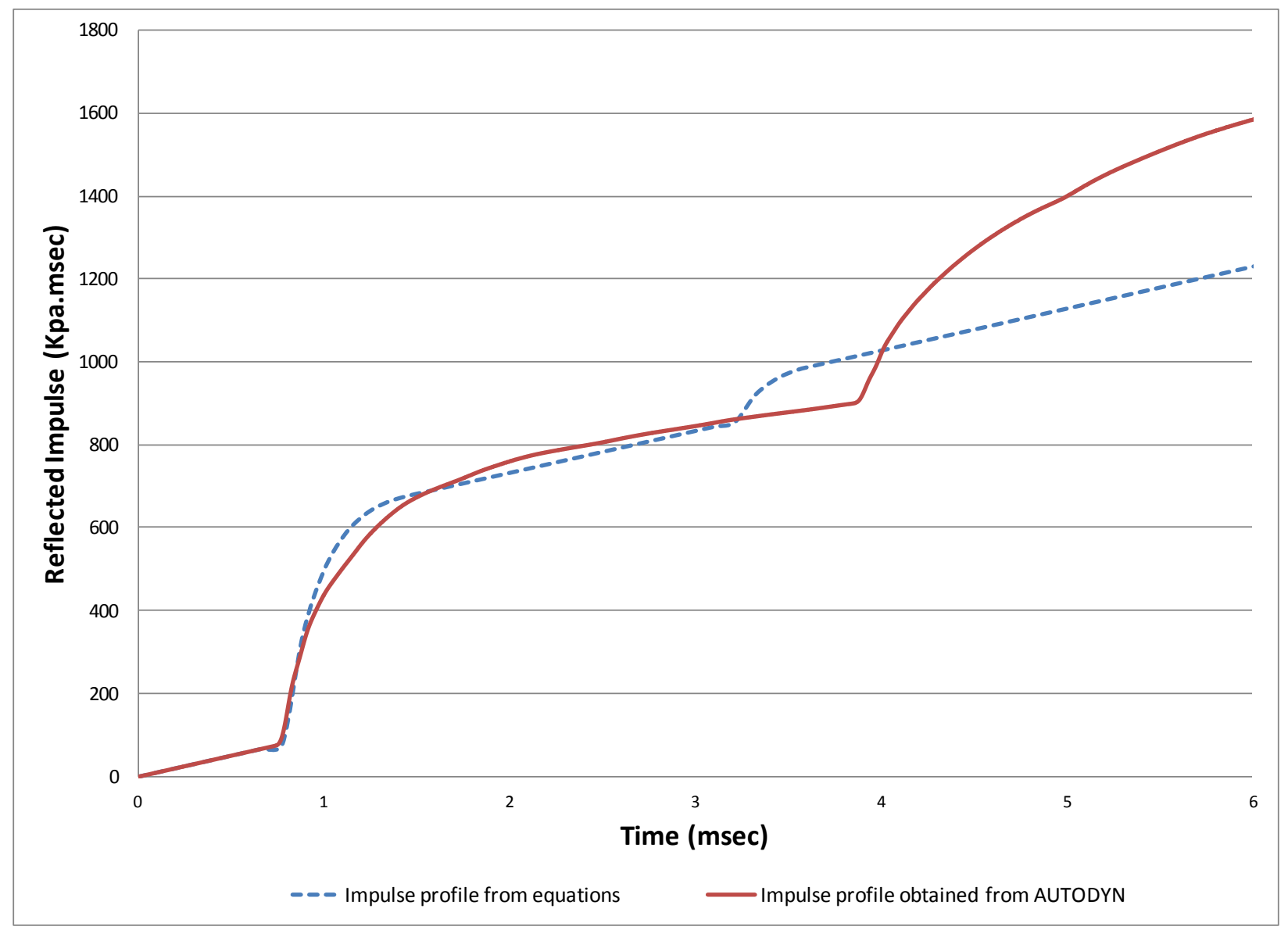

Figure 73: Impulse profile comparison between AUTODYN and proposed equations for $1 \mathrm{~kg}$ TNT in a $(3.0 \times 3.0 \mathrm{~m})$ container with vent ratio equals 0.25

\subsection{Summary}

In this chapter, the reflected pressure profiles on plates or angles forming the side wall of the VSS container were investigated. The pressure profile depends on the charge weight, vent area ratio and the size of the container.

The Friedlander waveform equation was used to predict the pressure profile on the VSS elements. However, the equation was originally developed to predict the pressure in case of free field blast problems but not internal explosion blast cases. A few modifications were applied to Friedlander waveform equation in order to calculate the pressure profile on the side walls of 
vented structures. The pressure profiles obtained from the modified equations were then compared with the pressure profiles obtained from AUTODYN and a good correlation was found between the two cases.

For VSS configurations that consist of angles, the pressure profile was different than that of the plates, so a geometric coefficient was introduced to take into account the effect of the different geometric configurations to predict the pressure and impulse values on the angles.

In Chapter 6, the structural response of plates and angles will be addressed. The developed equations that were introduced in this chapter 5 to calculate the pressure profile on steel members were used to apply the blast load on the steel members to study their structural behavior. 


\section{Chapter 6: Structural Design of VSS}

\subsection{Introduction}

This chapter investigates the structural response of steel VSS section subjected to internal explosion. Determining the response of a structure subjected to an internal explosion is challenging as it requires the combination of two disciplines; structural dynamics and fluid dynamics. Combining both disciplines is considerably complex. For the structural dynamics discipline, it is expected that the structural elements would have large deformations due to the load intensity, with corresponding nonlinear material behaviour, including possible fracture. While for the fluid dynamics problem, as the structure is loaded significant displacements may occur, and thus the flow boundaries are dynamically modified in contrast with classical fluid dynamics where the boundaries remain fixed through the fluid-structure interaction (Kivity, 1993). AUTODYN software as well as some other finite element packages have the capability to determine the structural response of structural elements due to blast loading however, the computation time for the simulation is long as well as it is expensive to use these software packages.

\subsection{Verification of AUTODYN model}

In this thesis, AUTODYN software was used to study the structural response of the steel sections of VSS. To verify the results obtained from the developed AUTODYN model, experimental data are required. Due to the lack of experimental data in this specific point of research, regular experimental work done by Olson (1993) was used to verify the structural response obtained from AUTODYN. The experimental work done by Olson was to investigate the response of $90 \times 90 \mathrm{~mm}$ square steel plates subjected to blast loading. The thickness of the steel plates was $1.5 \mathrm{~mm}$. Figure 74 shows the 3D AUTODYN model that was developed to simulate the experimental work carried out by Olson (1993). Only a quarter of the plate was modelled and symmetry boundary condition was applied at the plane of symmetry. The steel plate was modelled as a shell element and the properties of steel defined in the AUTODYN model were the same as the properties used in the experimental work. 
In the experimental work, the steel plates were fixed at its corners, so fixed boundary conditions were applied to the corners of the plate as shown in Figure 74. Olson studied the displacement at the midpoint of the plate; therefore in the AUTODYN model, Gauge 1 was located in the middle of the steel plate to track the midpoint deflection of the steel plate due to the applied blast load. The vectors in the figure simulate the blast load vectors on the steel plate.

In Chapter 4, it was found that the optimum mesh size to be used to simulate the air domain is $10 \mathrm{~mm}$, so this mesh size was used in the 3D model. For the steel plates, another sensitivity analysis was carried out to determine the appropriate mesh size to be used. The experimental setup made by Olson was modelled using different mesh sizes for the steel plate. From the results, a mesh size equals to $3 \mathrm{~mm}$.

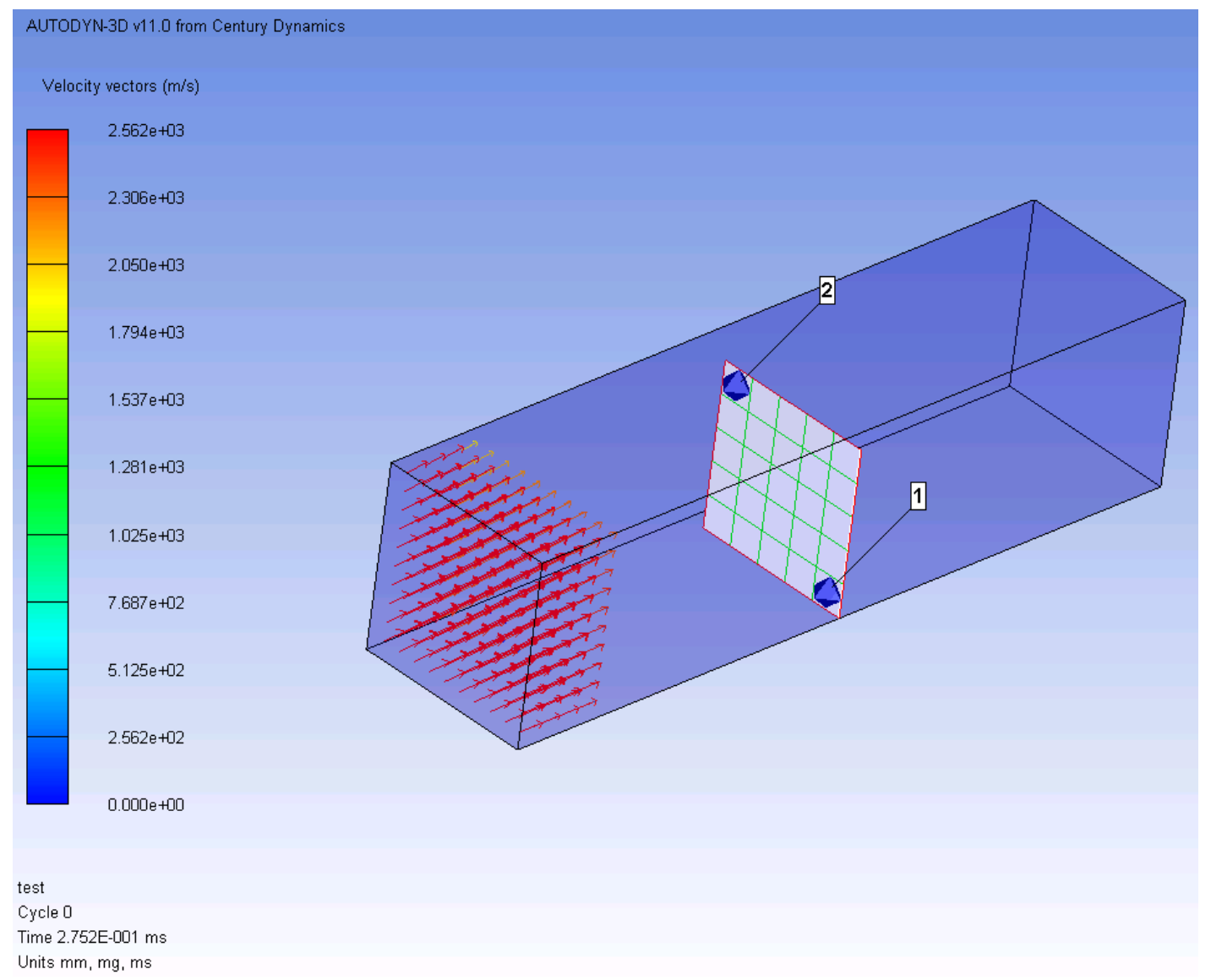

Figure 74: 3D model simulating a steel plate under blast load

The deflection of the steel plate at midpoint was recorded and compared to those obtained from the experimental work. Figure 75 shows the deflection of the plate midpoint obtained from 
the experimental work and the deflection of the corresponding point obtained from AUTODYN model (Gauge 1). A good correlation between the experimental and the AUTODYN results could be observed by comparing the two curves.

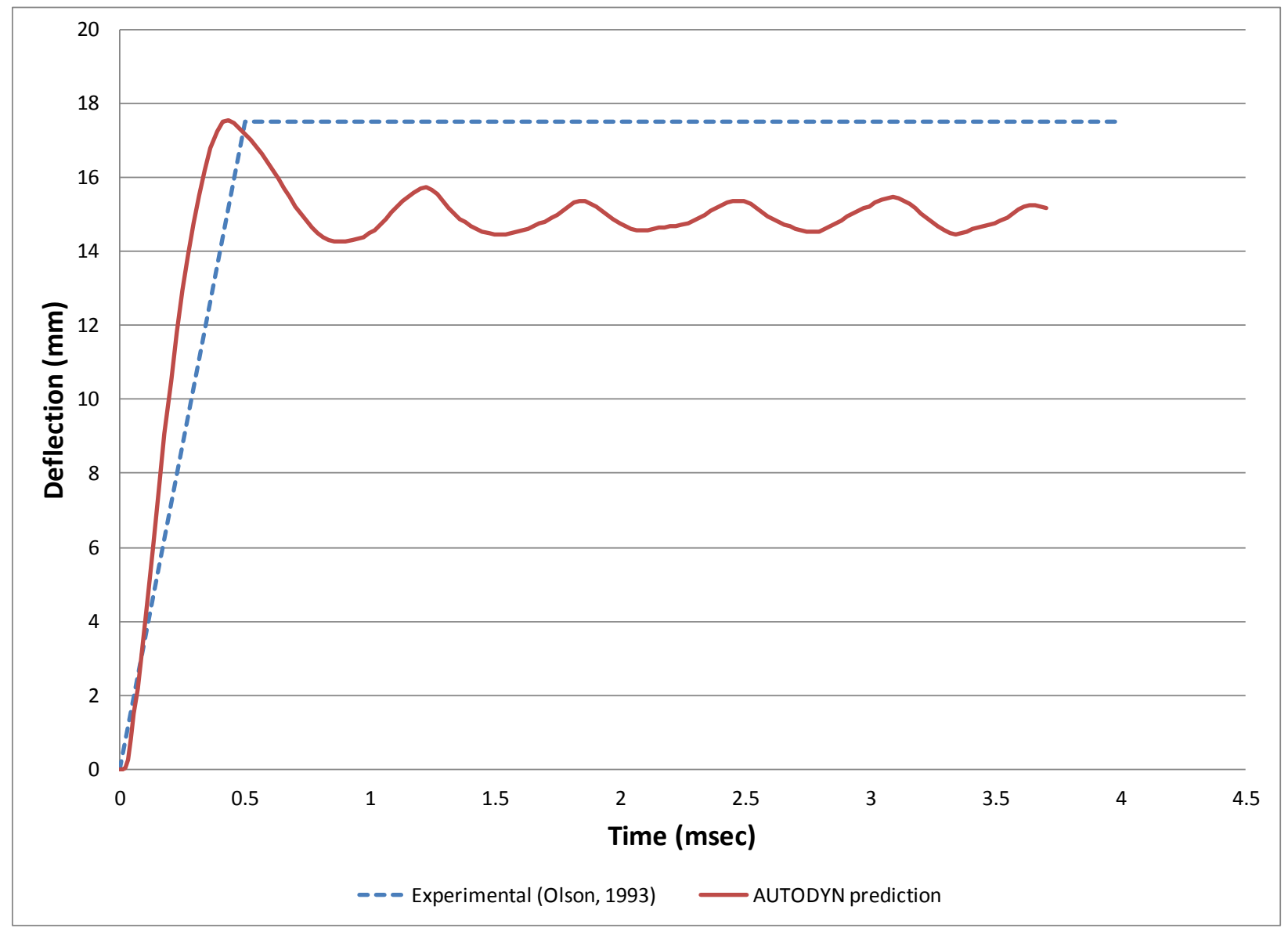

Figure 75: Plate midpoint displacement response obtained from AUTODYN (Gauge 1)

After verifying the 3D AUTODYN model, the same technique that was used in this model was used in the modelling of the VSS containers as well as the internal explosion.

\subsection{Single Degree of Freedom Method}

Another way to determine the dynamic response of structural members due to blast loads is using analytical approach. The basic analytical model used in most of loaded members' cases is the single-degree-of-freedom (SDOF) system. In this model, the structural components 
subjected to an external load were modeled as equivalent SDOF mass-spring systems with a nonlinear spring as shown in Figure 76.

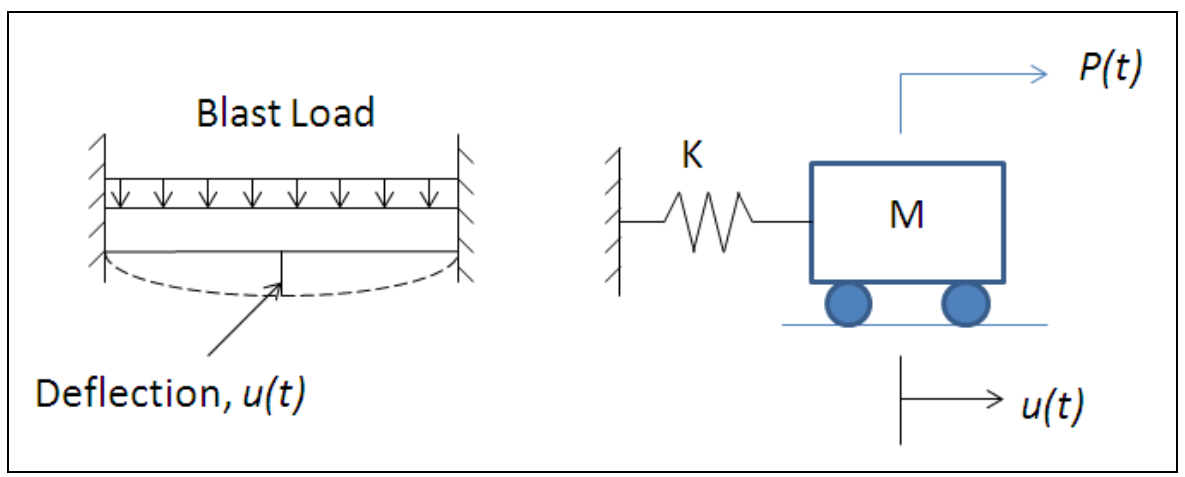

Figure 76: Beam subjected to blast load and Equivalent Spring-Mass System

The general equation of motion used to describe the dynamic response of the steel member subjected to blast loading is presented as follows:

$$
m \ddot{u}+c u+k u=P(t)
$$

Equation 32

where $m$ is the mass of the steel angle, $\ddot{u}$ is the acceleration, $c$ is the damping coefficient, $u$ is the velocity, $k$ is the stiffness of the element, $u$ is the displacement and $P(t)$ is the load acting on the element.

The equivalent independent ordinate $u$ was taken as the lateral displacement at the midspan of the structural members. Blast load is time dependent loading so the effect of the blast load on the structural members is considered to be a time dependent problem. Therefore the equation of motion is solved at each time step using the differential form of equation of motion as in Equation 33:

$$
m \Delta \ddot{u}+c \Delta \ddot{u}+k^{*} u-\Delta p=0
$$

Equation 33

where $\Delta \ddot{u}$ is the change in acceleration, $\Delta u$ is the change in velocity, and $\Delta p$ is the change in the applied load at each time step and $k^{*}$ is the effective stiffness. 


\subsubsection{Numerical integration}

Since the blast problem is a time transient problem, the equations should be solved at each time interval $\Delta t$. This is known as step-by-step integration method. Two of the most popular methods are the constant acceleration method and the linear acceleration method. As can be implied from the names, the constant acceleration method assumes that the acceleration is kept constant along the loading time while the linear acceleration method assumes that the acceleration changes linearly. The constant acceleration method is considered easier but less accurate than the linear acceleration method (Paz, 1980). In the developed SDOF model, the linear acceleration method was used. The linear acceleration integration method is explained in details in the next section.

\subsubsection{Linear acceleration step-by-step method}

Linear acceleration method is a second order differential equations method that is used in the numerical integration of the SDOF. The method is based on the assumption that the response acceleration varies linearly within a given time step. As a result, the response velocity is of a quadratic form and the response displacement of a cubic form as shown in Equation 35 and Equation 36. In this method, the material properties of the system $c_{i}$ and $k_{i}$ may include different forms of nonlinearities. So they are evaluated at the beginning of each time step, however, they remain constant until the next step. The only restriction in the analysis is that those coefficients; $c_{i}$ and $k_{i}$ are calculated at each time step and assumed to be constant within this time increment $\Delta t$. The linear acceleration method may be expressed as in Figure 77. 


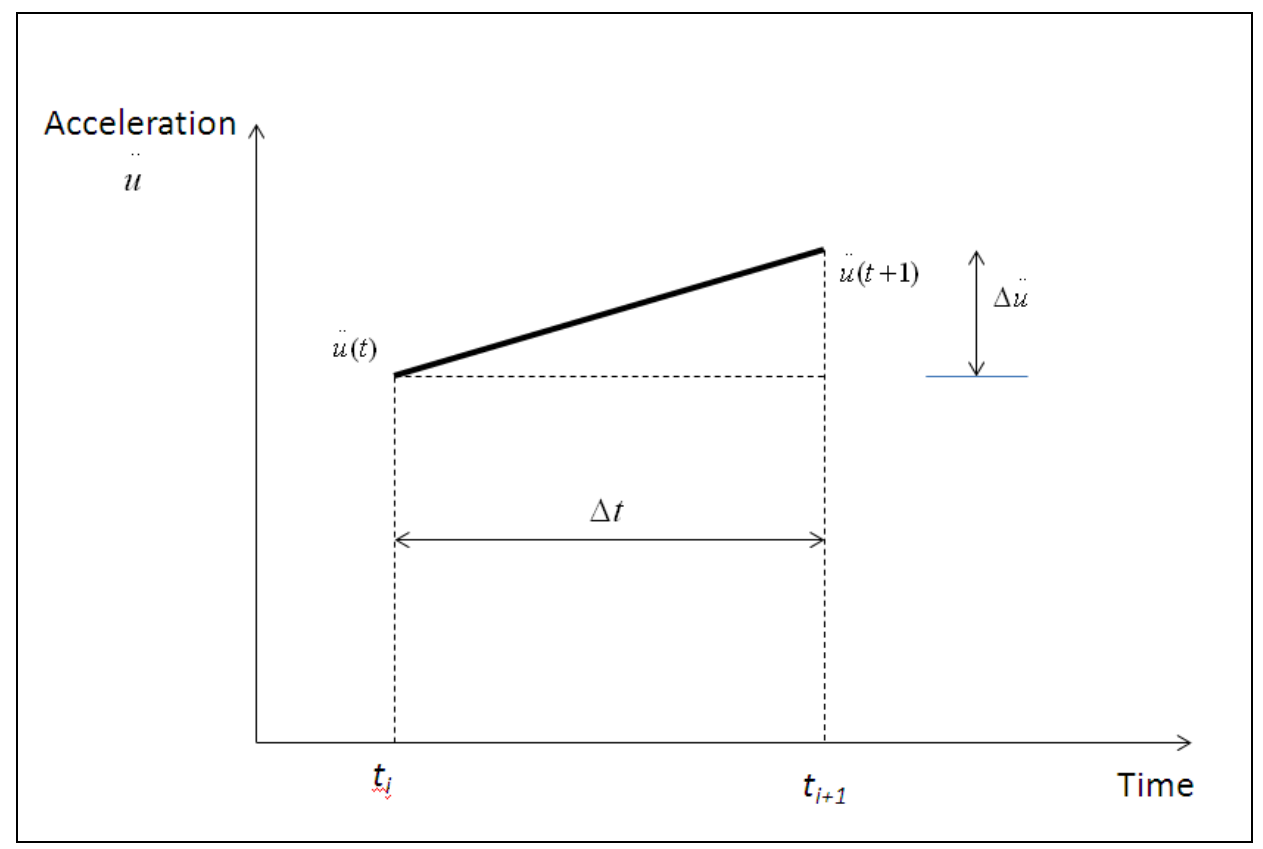

Figure 77: Assumed Linear variation of acceleration during time interval, (After Paz, 1980)

So assuming the acceleration to change linearly, the acceleration could be expressed as follow:

$$
\ddot{u}(t)=\ddot{u_{i}}+\frac{\Delta \ddot{u}}{\Delta t}\left(t-t_{i}\right)
$$

Equation 34

And by integrating Equation 34 once, Equation 35 is obtained representing the velocity of deformation and integrating it once more, Equation 36 is obtained indicating the displacement.

$$
\begin{gathered}
u(t)=\dot{u}_{i}+\ddot{u}_{i}\left(t-t_{i}\right)+\frac{1}{2} \frac{\Delta \ddot{u}}{\Delta t}\left(t-t_{i}\right)^{2} \\
u(t)=u_{i}+\dot{u}_{i}\left(t-t_{i}\right)+\frac{1}{2} \ddot{u}_{i}\left(t-t_{i}\right)^{2}+\frac{1}{6} \frac{\Delta \ddot{u}}{\Delta t}\left(t-t_{i}\right)^{3}
\end{gathered}
$$

Equation 36 
Substituting with Equation 34, Equation 35 and Equation 36 in Equation 33 leads to Equation 37.

$$
\Delta P_{i}^{*}=\Delta P_{i}+m\left[\frac{6}{\Delta T} \dot{u}+3 \ddot{u}\right]+c_{i}\left[3 \dot{u}+\frac{\Delta T}{2} \ddot{u}\right]
$$

Equation 37

where $\Delta \mathrm{P}_{\mathrm{i}}^{*}$ is the effective increment loading. The stiffness of the steel member is calculated at each time step by using the following equation. These set of equations were used in the developed SDOF model (Paz, 1980).

$$
\begin{array}{cr}
k_{i}^{*}=k_{i}+\frac{6 m}{\Delta T^{2}}+\frac{3 c_{i}}{\Delta T} & \text { Equation 38 } \\
\ddot{u}_{i+1}=\frac{1}{m}\left[P\left(t_{i+1}\right)-c_{i+1} u_{i+1}-R\right] & \text { Equation 39 }
\end{array}
$$

Where $R$ is the resistance of the member at a given time step. It is assumed to be $R_{i}=k_{i}{ }^{*} u_{i}$.

\subsubsection{Time interval}

As mentioned earlier, a blast load problem is a time transient problem. Moreover, blast loads are characterized by very high speed that makes the choosing of the time step critical since it has a big effect on the obtained results. It is very important for the time step to be suitable to track the peak pressure in the pressure profile. Also, the speed of the blast load depends on the charge weight and thus the time interval should match the speed of the blast wave that corresponds to the charge weight.

For the SDOF, there is another factor that needs to be satisfied to avoid inaccurate results for the element response. This factor is one of the restrictions for the use of the linear acceleration theory. It has been found from earlier studies, that the minimum time step to be used is $t / 10$ (Paz, 1980) where $t$ is the loading time where the load is applied. The other factor is the time step that was used should be small enough to predict the pressure profile to verify the results obtained from the proposed SDOF model, the results should be compared with those obtained from AUTODYN. So the other factor is to try to use the same time step used in 
AUTODYN in order to obtain a close pressure profile. In AUTODYN, the time step is calculated depending on the mesh size used in the model (Fairlie, 1998).

In this study, the time interval was equal to one hundred of the positive phase duration of the blast load $t_{d} / 100$. This is to satisfy the previous two requirements.

\subsubsection{Introduction to thin plates}

The steel sections introduced in this study are made of steel plates and L-shaped steel angles. The structural behaviour of the steel members depends on their dimensions or in other words, the ratio between the length of the steel section, $a$, to its thickness, $h$ plays a role in the structural behaviour of the steel plates and angles. Depending on this ratio, different theories may be applied to study the structural response of the steel members. The three main theories that could be used are thick plate theory, thin plate theory and membrane theory. Figure 78 shows the limits that judge when each of the three theories is applicable to the steel sections. If the ratio between the two dimensions is less than 8 , it is considered to be a thick plate. If the ratio lies between 8 and 80, then it is considered to be a thin plate. If it varies between 80 and100, then the membrane theory applies (Vantsel and Krauthammer, 2001).

It should be also noted that for a given steel member, the structural response could change from one condition to another. For example, it was found by previous researchers that the transition of a steel plate from bending to membrane response occurs when the deflection reaches $3 / 4$ of the thickness of the plate (Lee and Wierzbicki, 2005). When the plate deflection reaches approximately plate thickness, the membrane action takes over the bending action of a clamped plate. It should be noted that if the plate is not restrained at the end supports, then the assumption of experiencing an axial load on the membrane is no longer valid, and a separate solution must be developed (Lee and Wierzbicki, 2005). 


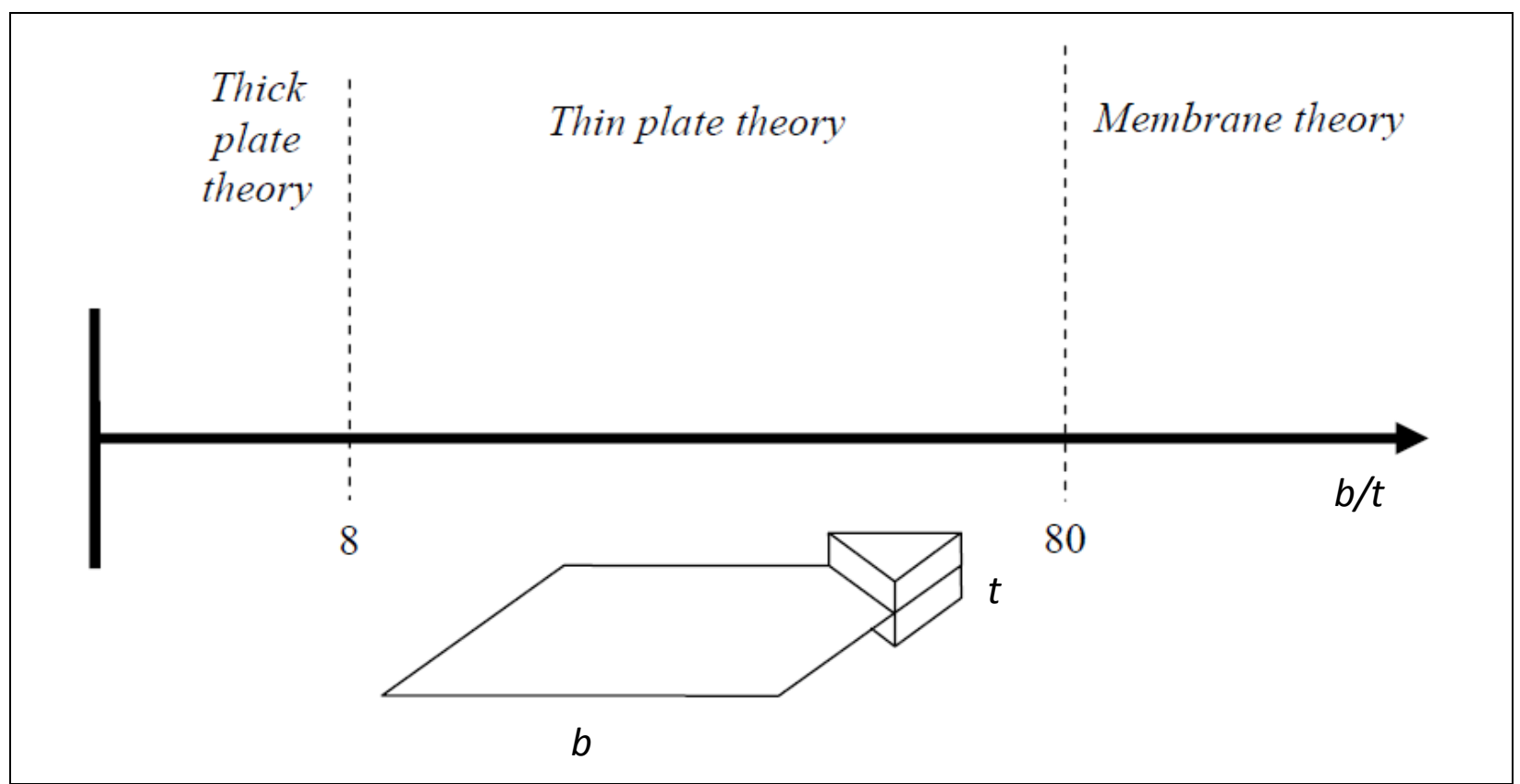

Figure 78: The distinguishing limits separating thick plate, thin plate, and membrane theory, (Vantsel and Krauthammer, 2001)

\subsubsection{Kirchhoff plate theory}

The range of steel plates that is being modelled in this study has the ratio that makes the steel plates lie in the thin plate's zone and thus the thin plate theory was applied. The thin plate theory is also called the "Kirchhoff" plate theory. Figure 79 shows the principal assumption when modelling a plate using Kirchhoff plate theory.

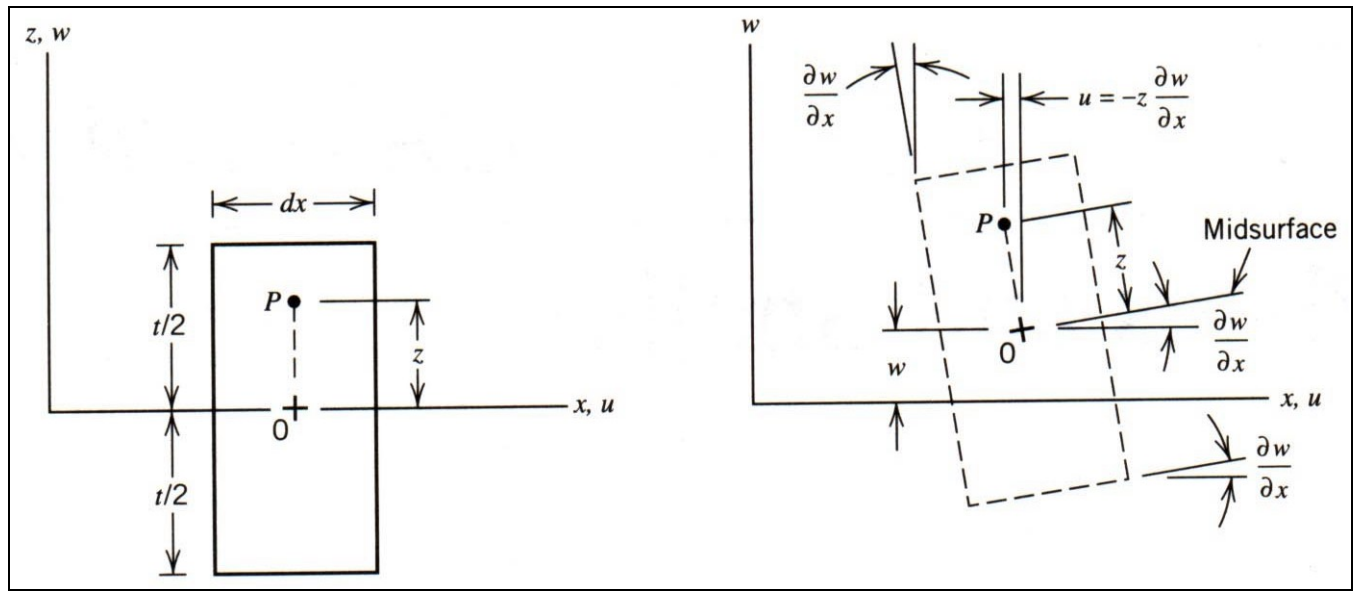

Figure 79: A differential slice from plate (Kirchhoff plate theory) 
Kirchhoff plate theory, the strain in the thickness direction for thin plates (Z-direction), is assumed to be 0 . Therefore, Equation 40 and Equation 41 can be used to calculate the principal and shear stresses in the plates:

$$
\begin{gathered}
\left\{\begin{array}{l}
\sigma_{x} \\
\sigma_{y}
\end{array}\right\}=-z \frac{E}{1-v^{2}}\left[\begin{array}{ll}
1 & v \\
v & 1
\end{array}\right]\left\{\begin{array}{l}
\partial^{2} w / \partial x^{2} \\
\partial^{2} w / \partial y^{2}
\end{array}\right\} \\
\tau_{x y}=-2 z G \frac{\partial^{2} w}{\partial x \partial y}
\end{gathered}
$$

Equation 41

Also according to Kirchhoff plate theory, the flexural rigidity of thin plates is expressed by:

$$
D=E I=E\left[\frac{b t^{3}}{12\left(1-v^{2}\right)}\right]
$$

Equation 42

Where $v$ is the Poisson's ratio, $b$ is the width of the plate, $t$ is the plate thickness.

\subsubsection{Elasto-plastic Behavior}

When a structural member is subjected to an applied load the element experiences deformation corresponding to the applied load. In SDOF model, the structural member is allowed to yield plastically. The member start deforming elastically until it reaches the elastic limit in tension $\left(y_{e t}\right)$ (zone \#1 in Figure 80). Beyond this point the member starts acting plastically (zone \#2 in Figure 80). When the member is unloaded, the behaviour of the member is again elastic (zone \#3 in Figure 80) until another reverse load is applied on the member producing compressive plastic yielding (zone \#4 in Figure 80). This behaviour where the response of the member transforms from elastic to plastic and vice-versa is known as elastoplastic behaviour. Figure 80 shows the resistance-displacement curve for elasto-plastic. 


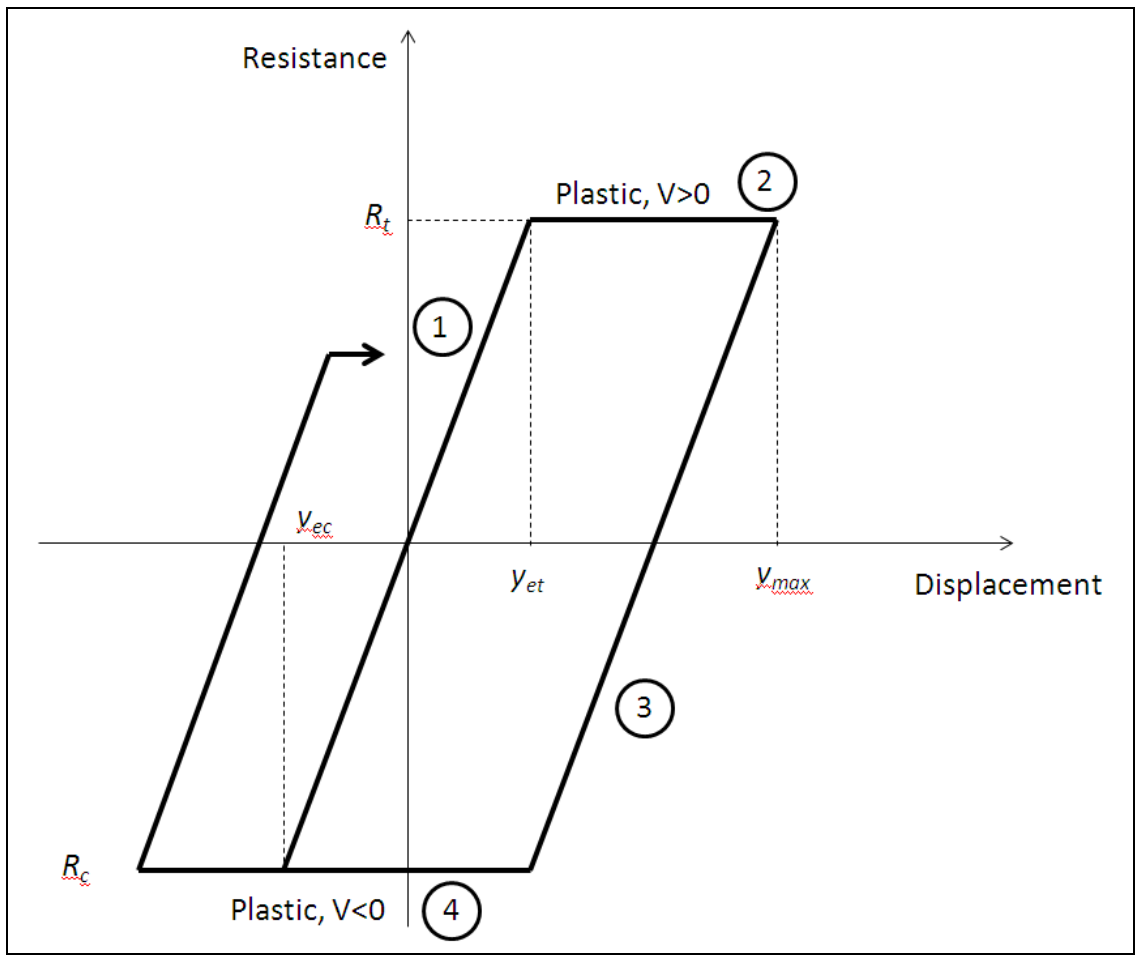

Figure 80: Force-displacement relationship for an elasto-plastic single degree of freedom

\subsubsection{Stiffness and Ultimate resistance}

In reality, the steel angles are welded to the main frame of the container. So the steel angles will be modelled as a fixed-fixed member in the SDOF model. The stiffness, $k$, and the ultimate moment resistance, $r_{u}$, for the steel angles were taken in accordance with UFC3-340-02 (2008). According to the UFC3-340-02 (2008), determining stiffness and resistance factors for one-way and two-way plate elements are based upon elastic deflection theory and the yield-line method. These methods are appropriate for defining the stiffness and ultimate load-carrying capacity of ductile structural steel plates. In applying these factors to steel plates, the modulus of elasticity should be taken equal to $29,600,000$ psi (204085 MPa). Table 12 shows the properties taken for the steel plates according to their loading condition; uniform and point loading. 
Table 12: Equivalent SDOF factors for beams with fixed ends

\begin{tabular}{|c|c|c|c|c|c|c|}
\hline $\begin{array}{c}\text { Loading } \\
\text { Diagram }\end{array}$ & Strain Range & $\begin{array}{c}\text { Load factor } \\
\left(K_{L}\right)\end{array}$ & $\begin{array}{c}\text { Mass } \\
\text { factor } \\
\left(K_{M}\right)\end{array}$ & $\begin{array}{c}\text { Load Mass } \\
\text { factor }\left(K_{L M}\right)\end{array}$ & $\begin{array}{c}\text { Maximum } \\
\text { Resistance, } R_{u}\end{array}$ & $\begin{array}{c}\text { Spring } \\
\text { Constants, } \\
(\mathrm{K})\end{array}$ \\
\hline \multirow{2}{*}{$\begin{array}{c}\text { Uniform } \\
\text { load }\end{array}$} & Elastic & 0.53 & 0.41 & 0.77 & $\frac{12 M_{P}}{L}$ & $\frac{384 E I}{L^{3}}$ \\
\cline { 2 - 7 } & Elasto-plastic & 0.64 & 0.50 & 0.78 & $\frac{8\left(M_{P}+M_{N}\right)}{L}$ & $\frac{384 E I}{5 L^{3}}$ \\
\hline
\end{tabular}

Where $E$ is the modulus of elasticity in MPa and $I$ is the moment of inertia of the section. For rectangular steel section, the moment of inertia is $b t^{3} / 12$ where $b(\mathrm{~mm})$ and $t(\mathrm{~mm})$ are the width and thickness of the section, respectively.

\subsubsection{Large Displacements and Membrane Forces "membrane action"}

When a section experiences bending stresses, the section starts to yield elastically. The stresses start at the edges of the section and reduce to zero at the neutral axis of the section as shown in Figure 81 (a). After reaching the yielding limit at the edges of the member, the rest of the section starts yielding and transition from elastic to plastic behaviour start taking place as shown in Figure 81 (b). By increasing the bending stresses, the whole section behaves plastically as in Figure 81 (c). Beyond this point, any more stresses will result in big deformations in the section and failure may occur.

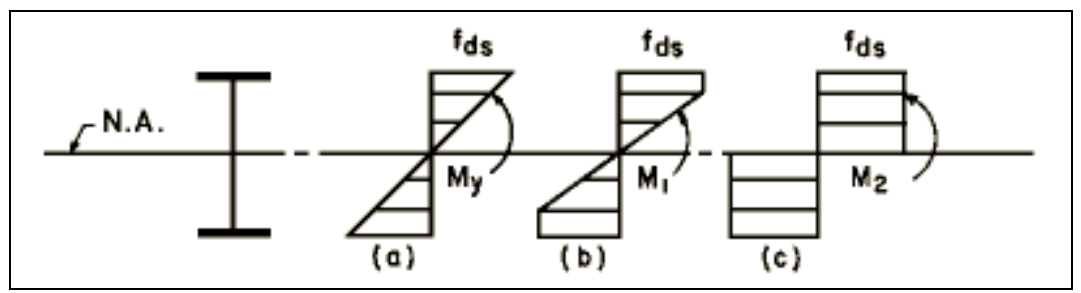

Figure 81: Theoretical Stress Distribution for Pure Bending at Various Stages of Dynamic Loading, (UFC3340-02, 2008) 
When dealing with blast loading, large deformations are expected to occur in the steel plates due to the applied load that makes the plate reaches the plastic limit. Two edge conditions may govern the deformation of plates in the plastic region. The first occurs when opposite edges are not built-in. In this case, elastic plate deflection theory and yield-line theory applies. The second involves tension-membrane action which occurs when at least two opposite edges are fixed or pinned. In this case, tensile-membrane action may occur once the member start yielding plastically (UFC3-340-02, 2008). This tensile membrane action is sometimes known as membrane (string) action where the membrane acts like a string.

In this study, the steel plates or angles of the side wall of the VSS container are assumed to be fixed connected to the main frame of the container. Therefore the steel members were assumed to be fixed-fixed members and therefore the tensile membrane action was taken into consideration. Figure 82 shows the tensile membrane actions that could occur in the steel plate due to large deformation.

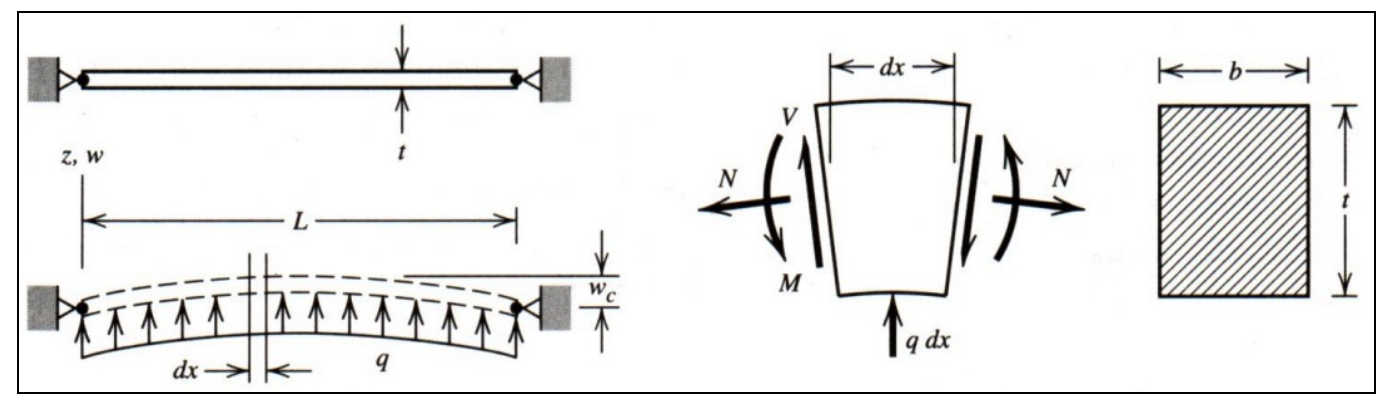

Figure 82: String action in thin plates due to large deformation (ASCE, 2011)

It was mentioned by previous studies that the membrane action starts occurring when the deflection of the steel plate is more than 0.75 to 1.5 the thickness of the steel plate (Seraji, 2013). And accordingly, the resistance of the section increases at this point when the tensile membrane action is taken into consideration. Equation 43 was introduced to predict the resistance of thin steel section due to the tensile membrane action assuming that the membrane action starts when the deflection is more than the thickness of the steel plate (ASCE, 2011). In this equation, the resistance of the section $\left(R_{u}\right)$ was assumed to be a function of the elastic resistance of the section $\left(R_{E}\right)$ depending on the deflection of the section $(\Delta)$. 


$$
R_{u}=\left(\frac{\pi}{2} \frac{\Delta}{t}\right) R_{E}
$$

Equation 43

Where, $\Delta$ is the midspan deflection, $t$ is the thickness and $R_{E}$ is the elastic resistance of the steel section.

\subsubsection{Damping}

Damping is an effect that reduces the amplitude of oscillations in an oscillatory system. Damping is represented by a factor known as damping ratio $(\zeta)$. This factor is a measure of how the system oscillation decays after a disturbance occurs to the system. Many systems exhibit oscillatory behavior when they are disturbed from their position of static equilibrium. Depending on the value of this damping ratio, the system can be one of the following:

- Overdamped $(\zeta>1)$ : The system decays exponentially to equilibrium without oscillating. Larger values of the damping ratio $\zeta$ return to equilibrium more slowly.

- Critically damped $(\zeta=1)$ : The system returns to equilibrium as quickly as possible without oscillating. This is often desired for the damping of systems such as doors.

- Underdamped $(0<\zeta<1)$ : The system oscillates (at reduced frequency compared to the undamped case) with the amplitude gradually decreasing to zero.

- Undamped $(\zeta=0)$ : The system oscillates at its natural resonant frequency $\left(\omega_{0}\right)$.

In this study, the system was assumed to be undamped where the system oscillates at its natural frequency. This is because the primary concern about the steel angles was the maximum deflection at mid span.

\subsubsection{Failure Criterion}

According to the UFC3-340-02 (2008), the failure of steel members occurs when the support rotation $(\theta)$ or the ductility ratio $(\mu)$ exceed a certain limit. Table 13 shows the failure limits for steel members according to UFC3-340-02 (2008). 
Table 13: Preliminary Design limits, UFC3-340-02 (2008)

\begin{tabular}{|c|c|c|}
\hline \multirow{2}{*}{ Deflection Magnitude } & \multicolumn{2}{|c|}{ Deformation } \\
\cline { 2 - 3 } & $\boldsymbol{\theta}_{\max }$ & $\boldsymbol{\mu}_{\max }$ \\
\hline Small & 2 & 5 \\
\hline Moderate & 4 & 10 \\
\hline Large & 12 & 20 \\
\hline
\end{tabular}

The calculated maximum dynamic deflection of the equivalent SDOF system is used to calculate the two maximum response parameters; the maximum support rotation and the ductility ratio. The portion of the span used to determine the support rotation for the structure member is always one-half the span length. This is true for fixed-simple beams even though the maximum deflection point for elastic response is not at mid-span. For this case, the maximum deflection is within approximately $15 \%$ of mid-span and moves closer to mid-span during elasto-plastic response. Since it is relatively complicated to determine the exact location, the maximum deflection point is assumed at mid-span when determining the support rotation. Figure 83 shows the deflection of a simply supported beam. The support rotation defined by $\theta$ and the deflection is given by the term $u$.

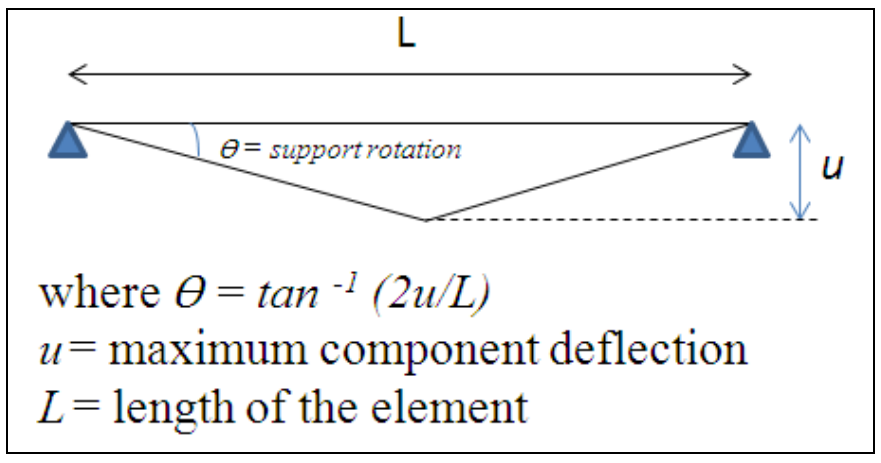

Figure 83: Deflection of simply supported beam

In regular design procedure, the maximum allowable ductility ratio for steel members is taken as equal to 10 , or end support rotations equals to 2 degrees. These limits are used in order not to reach the large deformation limits mentioned in Table 13. However, VSS containers are considered to be protective structures so big deformations are expected to occur in the structure. Therefore, in the design procedure, failure of the steel members was defined when the maximum 
allowable ductility exceeds 20 or end support rotations exceeds 12 degrees. Figure 84 shows the Resistance-Deflection curve where the deflection limits are defined as the UFC3-340-02 (2008).

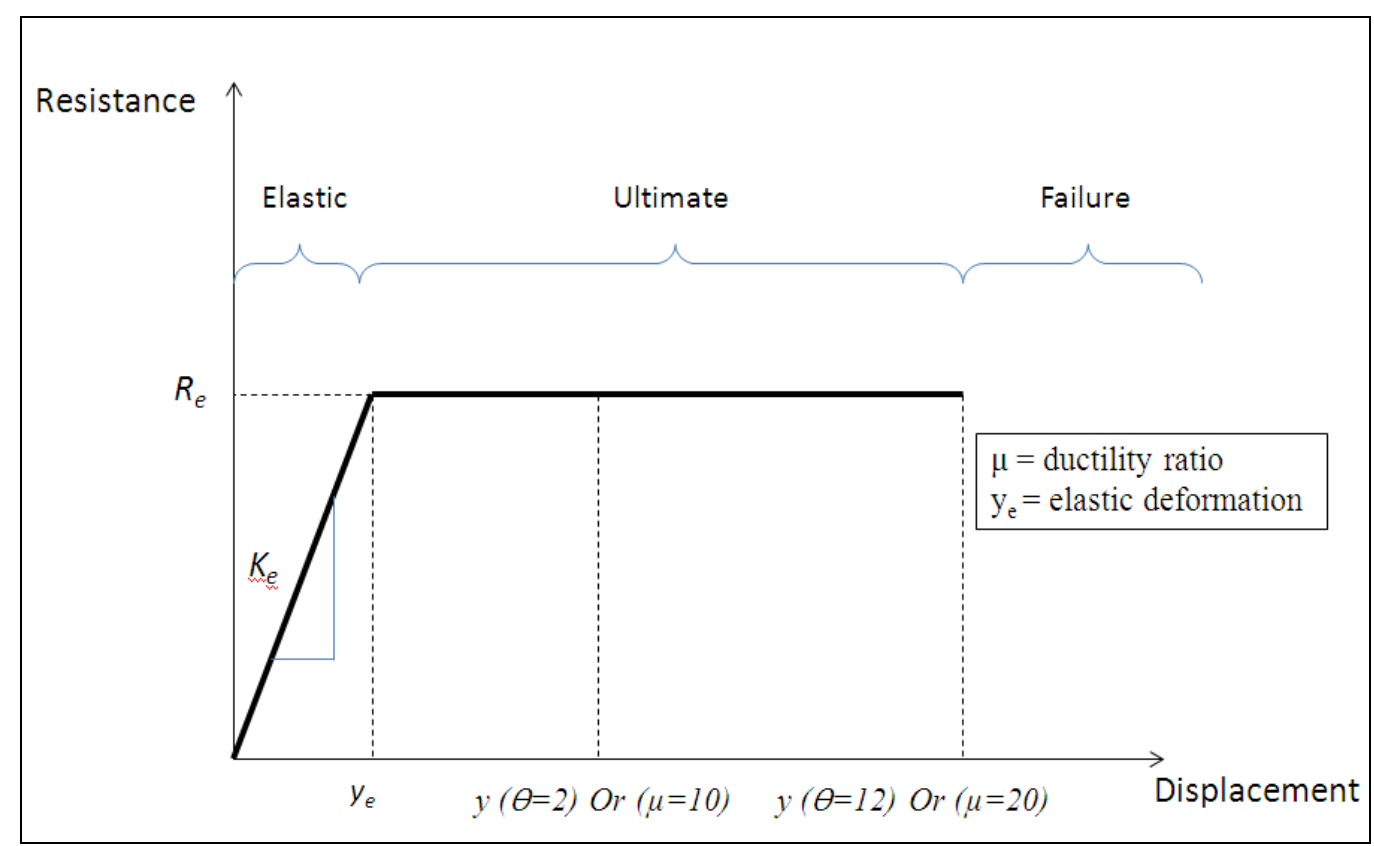

Figure 84: Resistance-Deflection curve for steel members

When large deformations start to occur in the steel member, the tension membrane action takes place. This occurs when the ductility ratio is more than 10 (end support rotations $=2$ degrees). In this zone, the resistance of the steel member increase due to the tensile membrane action until failure occurs to the beam when the ductility ratio reaches 20 (end support rotations $=12$ degrees). So the resistance-displacement curve could be derived as shown in Figure 85 . 


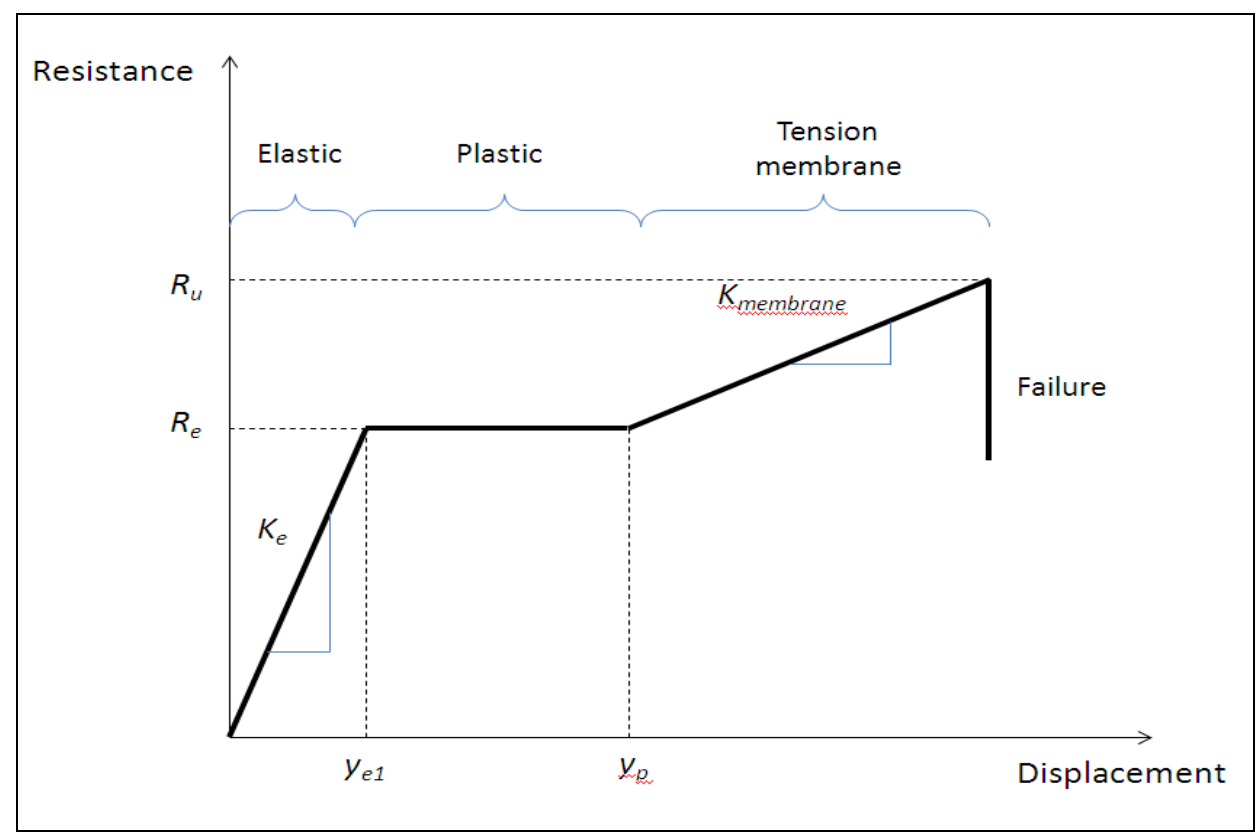

Figure 85: Stress strain curve for steel element showing the tensile membrane action

Note that the maximum allowable deflection in the steel member is calculated as the deflection that is corresponding to ductility ratio equals to 20 (end support rotations $=12$ degrees). For simplification, it was assumed that the tension membrane action start taking place in the member when the deflection of the member exceeds the elastic limit and the member starts behaving plastically. Therefore, the resistance-deflection curve will be assumed to be as shown in Figure 86. Appendix D shows the Alogarithm for step-by-step solution for elasto-plastic single degree of freedom system developed in this study. 


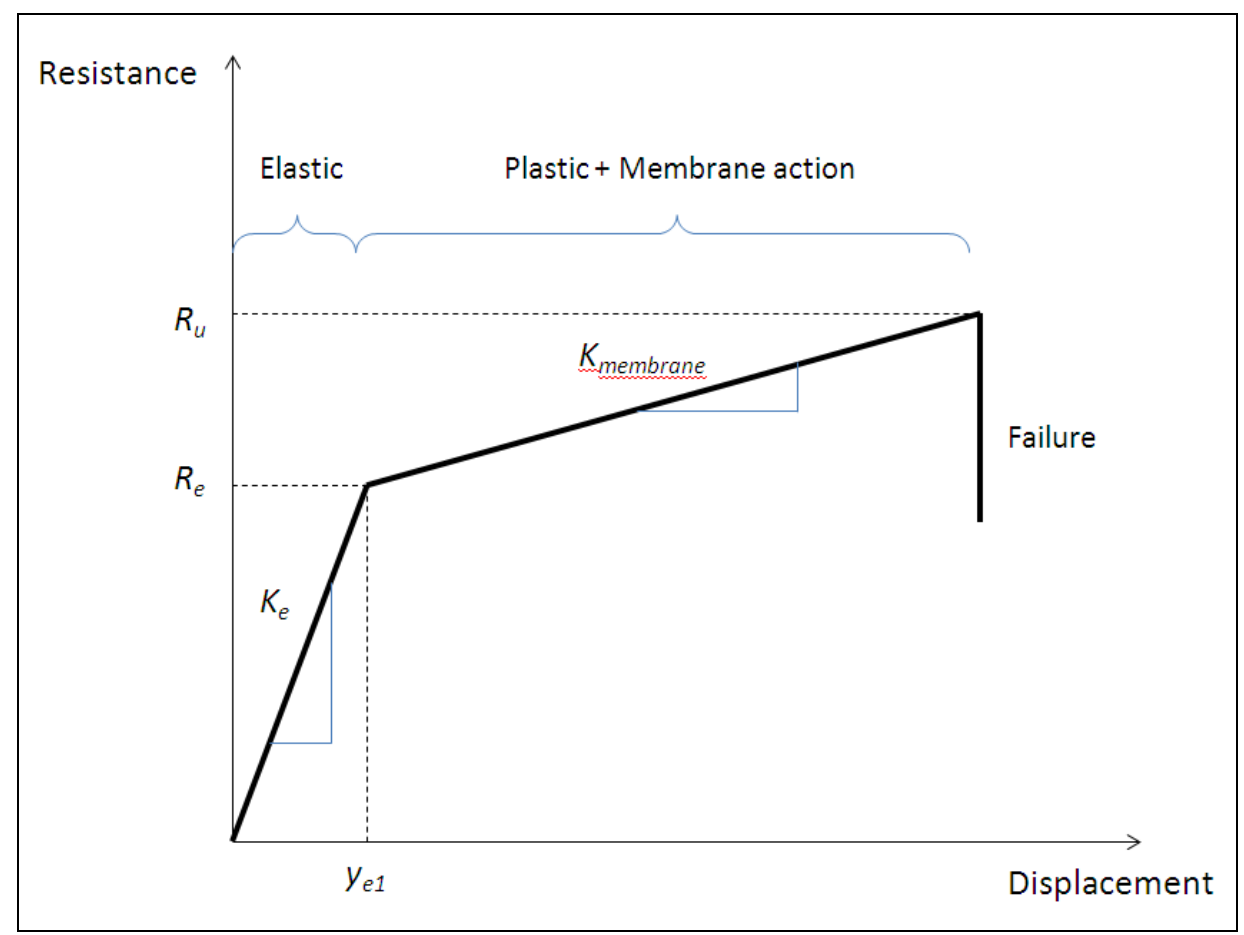

Figure 86: Simplified Resistance Curve with tensile membrane

\subsubsection{Pressure profile}

As mentioned in Chapter 4, the reflected pressure-time profile on the steel members changes from one case to another depending on the vent ratio of the structure as well as the orientation of the steel angle with respect to the blast wave. The obtained pressure profiles from the proposed equations mentioned earlier in Chapter 4 were used as an input to the SDOF model in order to study the structural response of the steel members due to internal explosion.

\subsection{Results obtained from SDOF model}

The results section is mainly a comparison between the structural response (displacement and velocity) of the steel angles obtained from AUTODYN and those obtained from the SDOF model. Different charges; 1, 5, 10 and $20 \mathrm{~kg}$ of TNT, as well as different container sizes; $2.0 \times 2.0,3.0 \times 3.0$ and $4.0 \times 4.0 \mathrm{~m}$, with different vent area ratios; 0.25 and 0.5 , were modelled. The responses of the steel plates were investigated for all the different studied cases. The deflection at the mid span of the steel plates was recorded in AUTODYN and compared to the results obtained from the SDOF model. This section presents some of the obtained results for different cases. The cases that will be addressed in this section are for different structural behaviours of 
steel plates; elastic, plastic, plastic with membrane action and failure in order to show the difference of those behaviours on the structural response.

Figure 87 shows an example for the output that is obtained from the 3D AUTODYN model. In the figure, the deformation of the steel angles can be seen as well as the stresses along the steel angles. In this model, the main frame of the container was kept as fixed in order to study the deformation of the steel angles as well as satisfy the fixed boundary conditions at the supports for the steel angles.

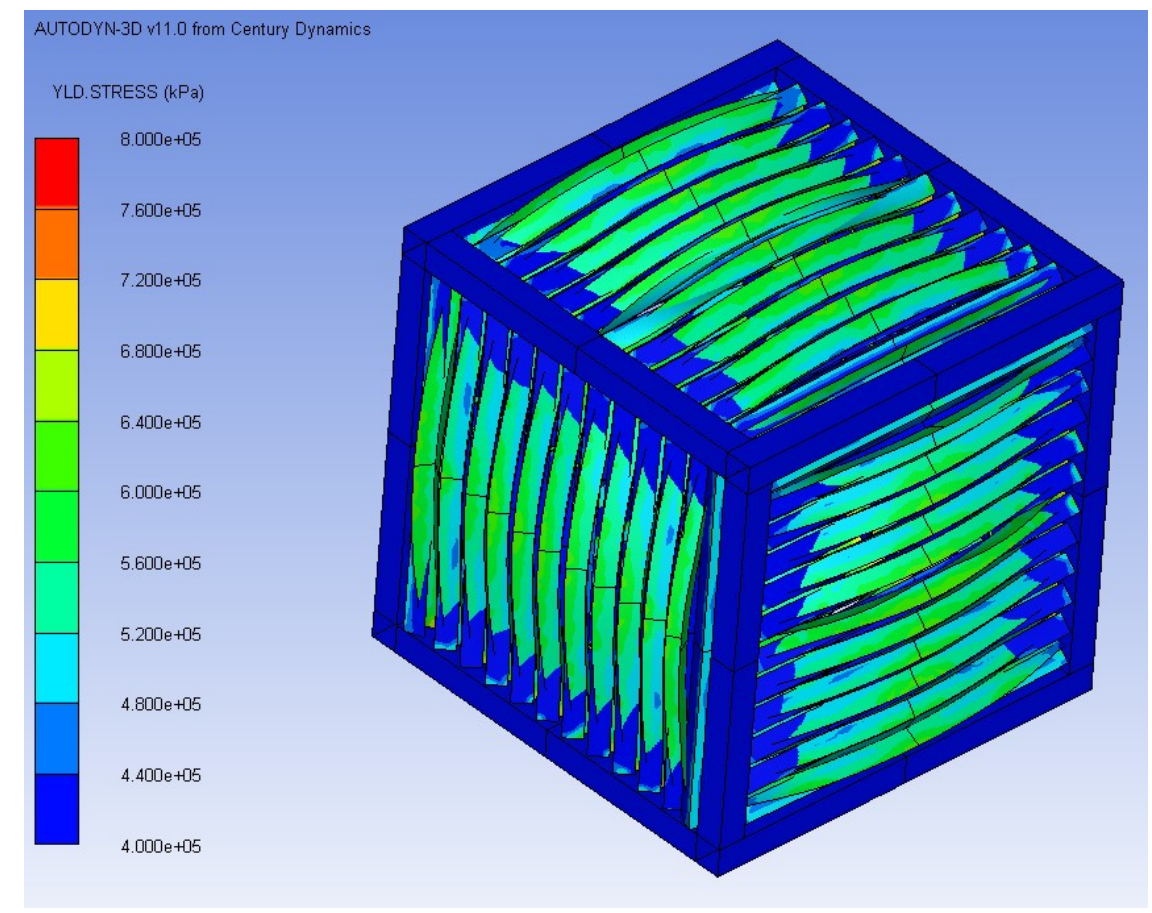

Figure 87: Stresses in 3D container

\subsection{1 $1 \mathrm{~kg}$ TNT charge in a $(2.0 \times 2.0 \mathrm{~m})$ container with 0.5 vent area ratio}

This case represents the behaviour of the steel plates for a vented Suppressive shield container of $2.0 \times 2.0 \mathrm{~m}$ size and with a vent ratio equals to 0.5 and subjected to a $1 \mathrm{~kg}$ of TNT charge in the center of the container. The thickness of the steel plates was chosen to be $50 \mathrm{~mm}$. In this case the steel plates were able to withstand the applied load without reaching the plastic zone. The deflection was recorded at the mid span of the steel plate and was found that the maximum deflection of the steel plate was $3.68 \mathrm{~mm}$ that is less than the allowable elastic deflection for the steel plate $(17.3 \mathrm{~mm})$. Figure 88 shows a comparison between the output from 
AUTODYN and the developed numerical model for the relation between the deflections at the mid span with respect to time.

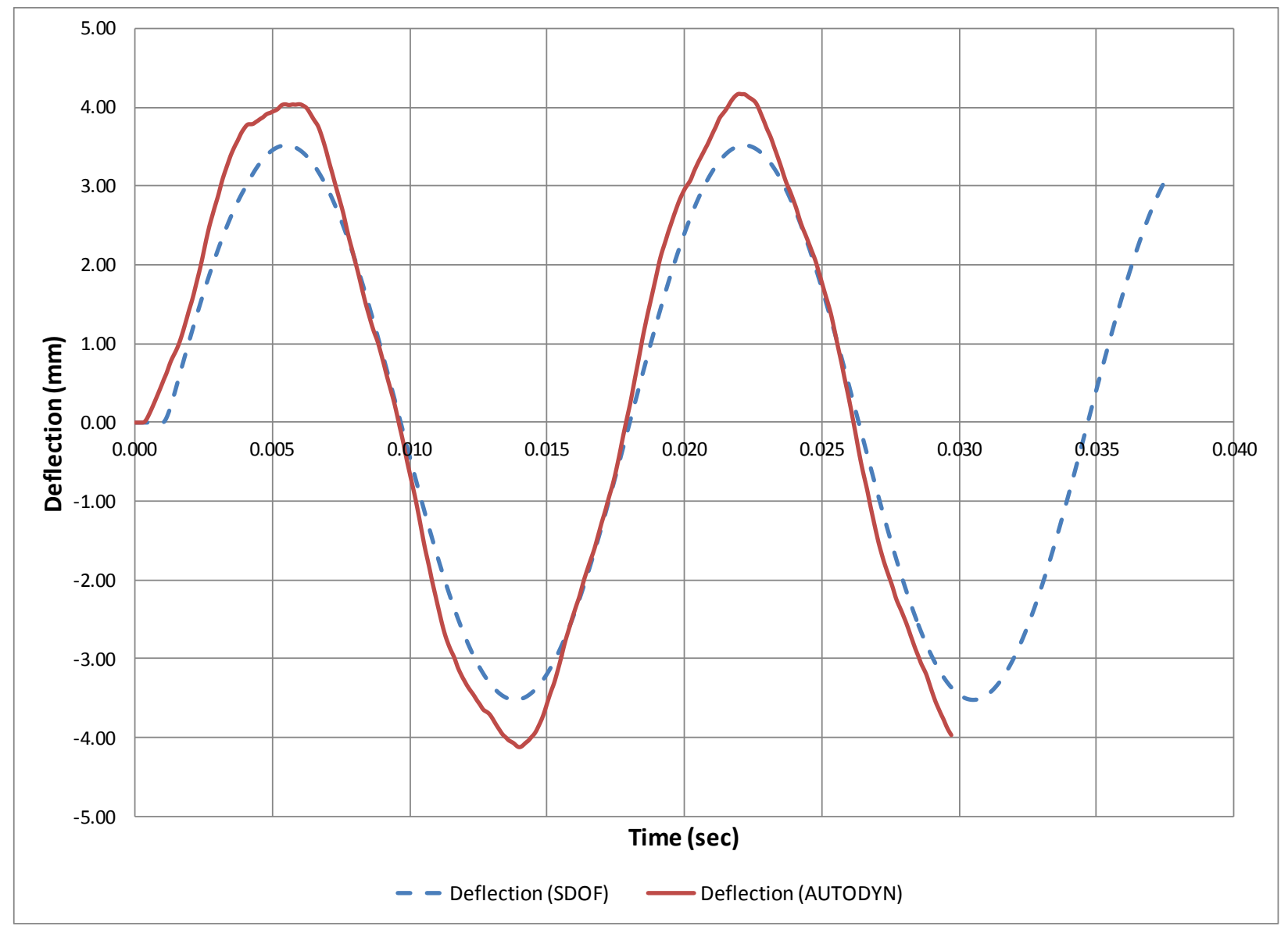

Figure 88: Comparison between obtained results from developed model and AUTODYN to predict displacement at mid span (50 $\mathrm{mm}$ thick)

The maximum deflection at the mid span of the angle was found to be $3.68 \mathrm{~mm}$ while the maximum deflection obtained from the 3D AUTODYN model was $4.1 \mathrm{~mm}$. Figure 89 shows the velocity at the mid span of the steel angle with respect to time for both obtained results from AUTODYN and from the numerical model. The developed SDOF model was able to obtain the velocities at mid span of the steel elements compared to that obtained from the AUTODYN as shown in Figure 89. 


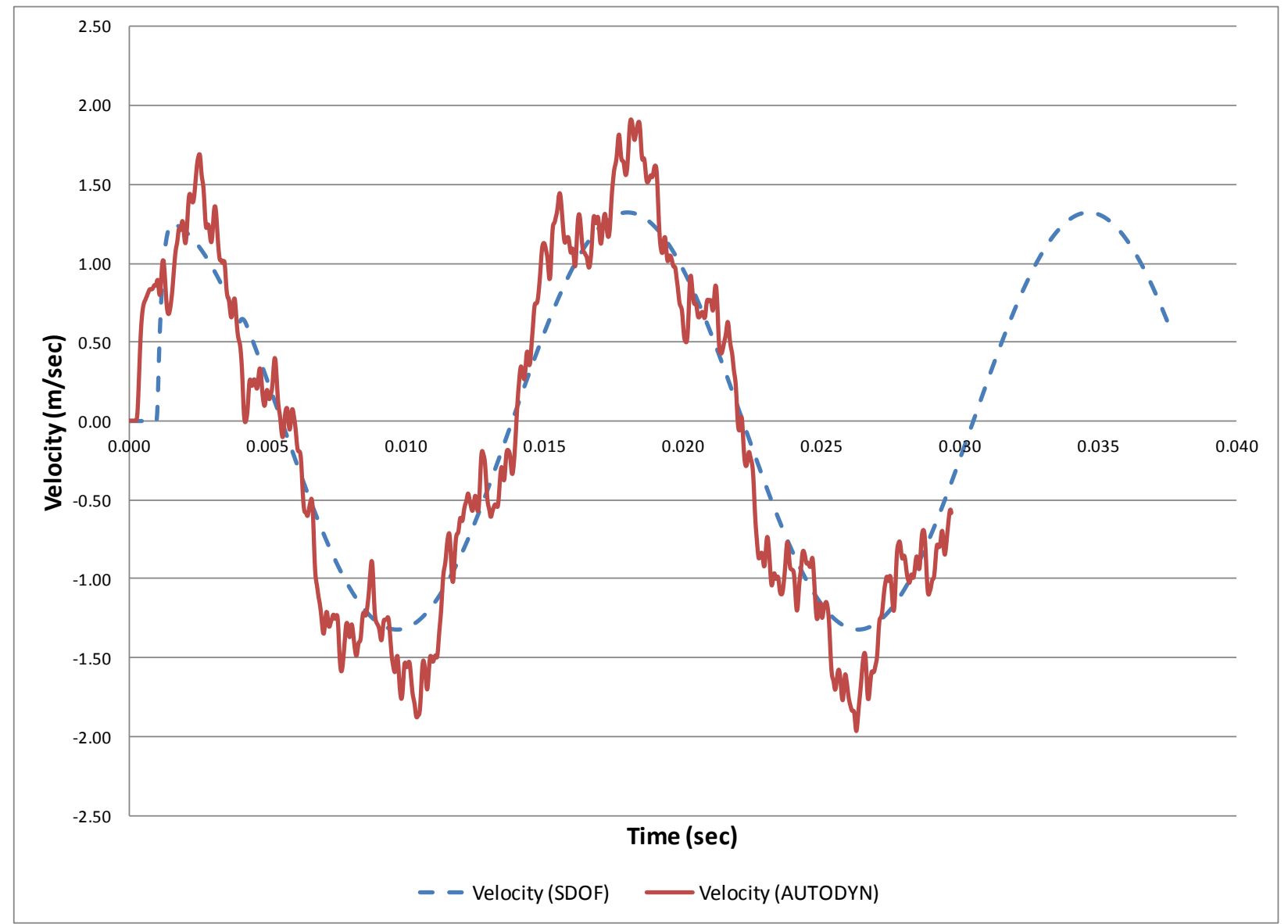

Figure 89: Comparison between obtained results from developed model and AUTODYN to predict velocity at mid span (50 mm thick)

To obtain a better understanding about the structural behaviour of the steel plates, the resistance-displacement curve is plotted as shown in Figure 90. The figure shows that the slope of the curve is constant that indicates that the plate is behaving elastically and no plasticity took place. This is because the thickness of the steel plate was over designed so the maximum deflection of the steel plate didn't exceed the elastic limit. 


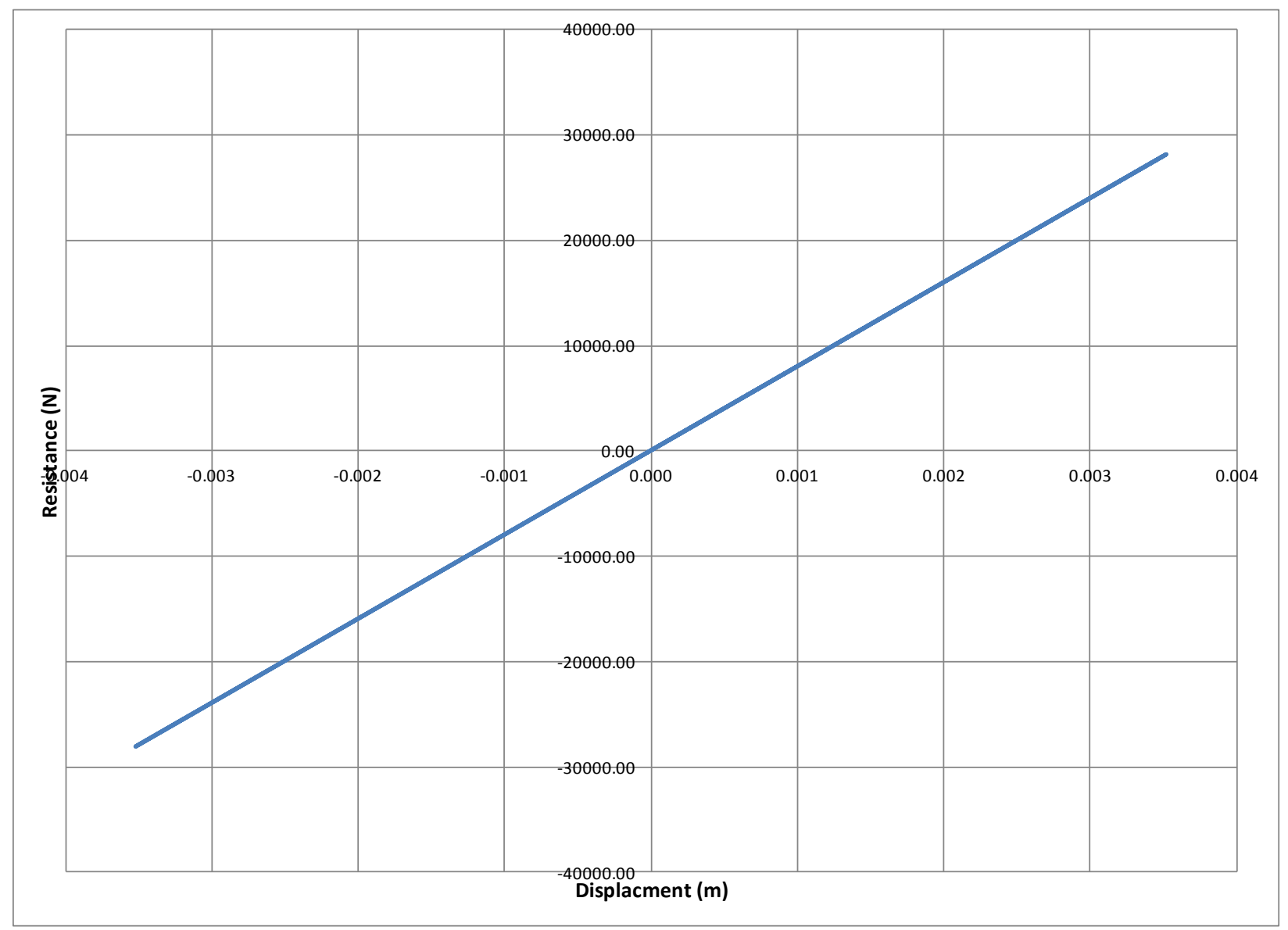

Figure 90: Resistance-Displacement curve (50 mm thick)

As previously discussed, the objective of this research is to obtain VSS containers that are transportable and to be able to contain an explosion inside. So the objective is to design the containers to be light in weight. Also, these structure are considered protective structures so the steel members of the side wall of the containers should be designed in a way that allow them to deform until they reach their plastic limit. So the next step was to try reducing the thickness of the steel plates and studying the behaviour of the steel plates due to the applied load.

\subsection{2 $1 \mathrm{~kg}$ TNT charge in a $(2.0 \times 2.0 \mathrm{~m})$ container with 0.5 vent area ratio (Plastic deformation)}

For the same previous case, the thickness of the plate was reduced to $10 \mathrm{~mm}$ instead of 50 $\mathrm{mm}$. Figure 91 shows the resistance-deflection curve for steel plate with $10 \mathrm{~mm}$ thick. 


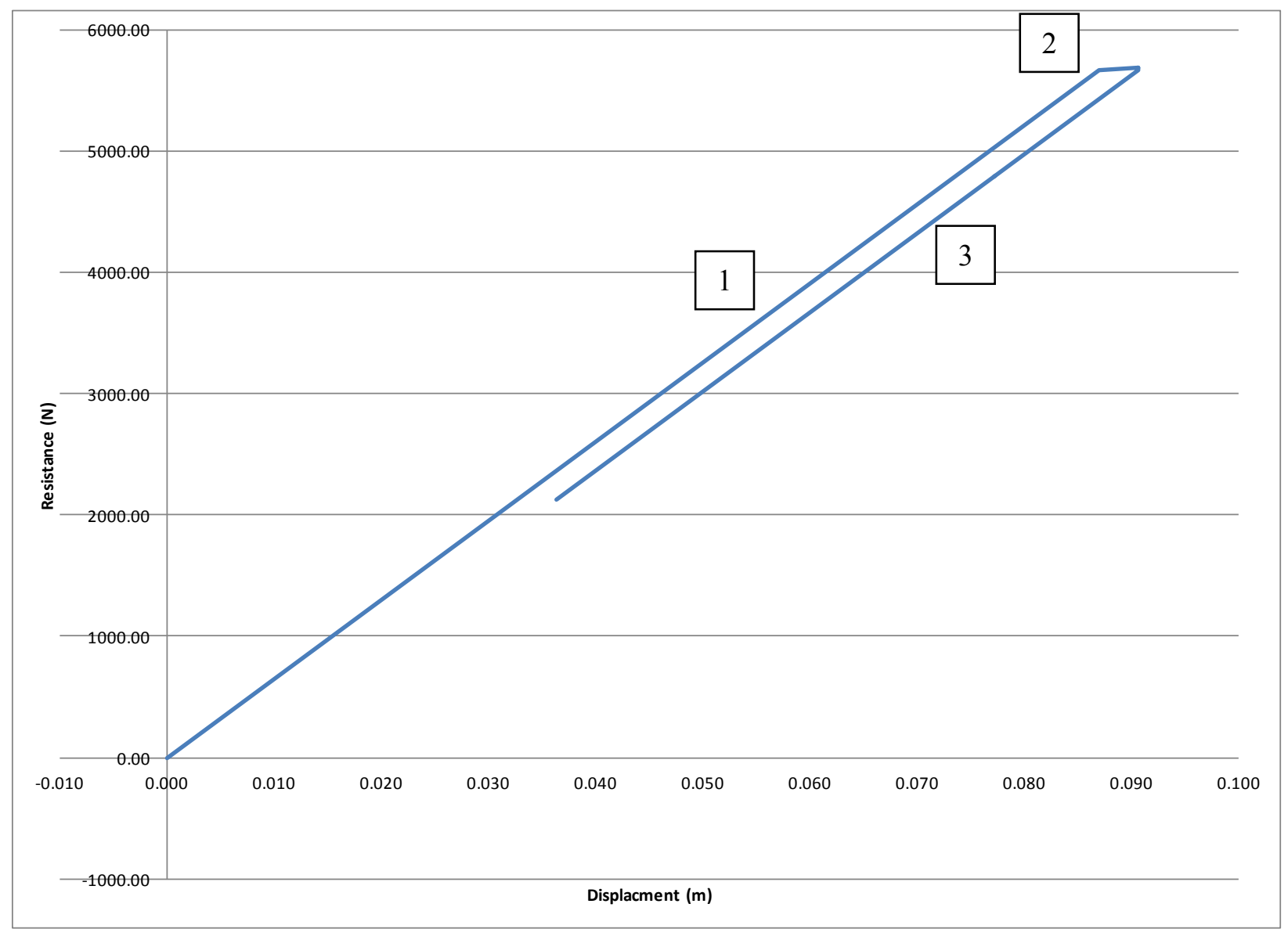

Figure 91: Resistance-deflection curve for steel plate (10mm thick)

It can be observed from the curve in Figure 91 that the steel plate behaved differently throughout the loading phase. The first zone in the curve (zone 1) simulates the elastic behaviour of the steel plate while the horizontal line (zone 2) indicates that the steel plate started behaving plastically. After the load passed the plate and the plate was unloaded, it returned back to elastic behaviour (zone 3). This curve indicates that the steel plate's deflection exceeded the elastic limit and thus the behaviour of the steel plate reached the plastic limit. If the thickness of the steel plate is reduced even more, tension membrane action starts taking place as will be shown in the next section. 


\subsection{3 $1 \mathrm{~kg}$ TNT charge in a $(2.0 \times 2.0 \mathrm{~m})$ container with 0.5 vent area ratio (Tension membrane action)}

Following up with the same example, the thickness of the steel plate was reduced from 10 to $8 \mathrm{~mm}$ thick. Figure 92 shows the resistance-displacement curve for this case. In this case, the steel plate started deforming elastically (zone 1). When the deflection of the steel plate exceeded the elastic deflection limit $\left(\Delta_{e}\right)$, the steel plate started behaving plastically and tension membrane action started taking place in the steel plate which increased the resistance capacity of the plate as indicated by the inclined line at zone 2 . The resistance in zone 2 is a combination of the ultimate resistance of the section and the resistance increase due to the membrane action. Zone 3 in the curve indicates the steel plate response when the blast wave passed the steel plate. The plate behaved again elastically. No failure took place in this case of loading; however, permanent deformation occurred in the plate due to reaching the plastic limit. 


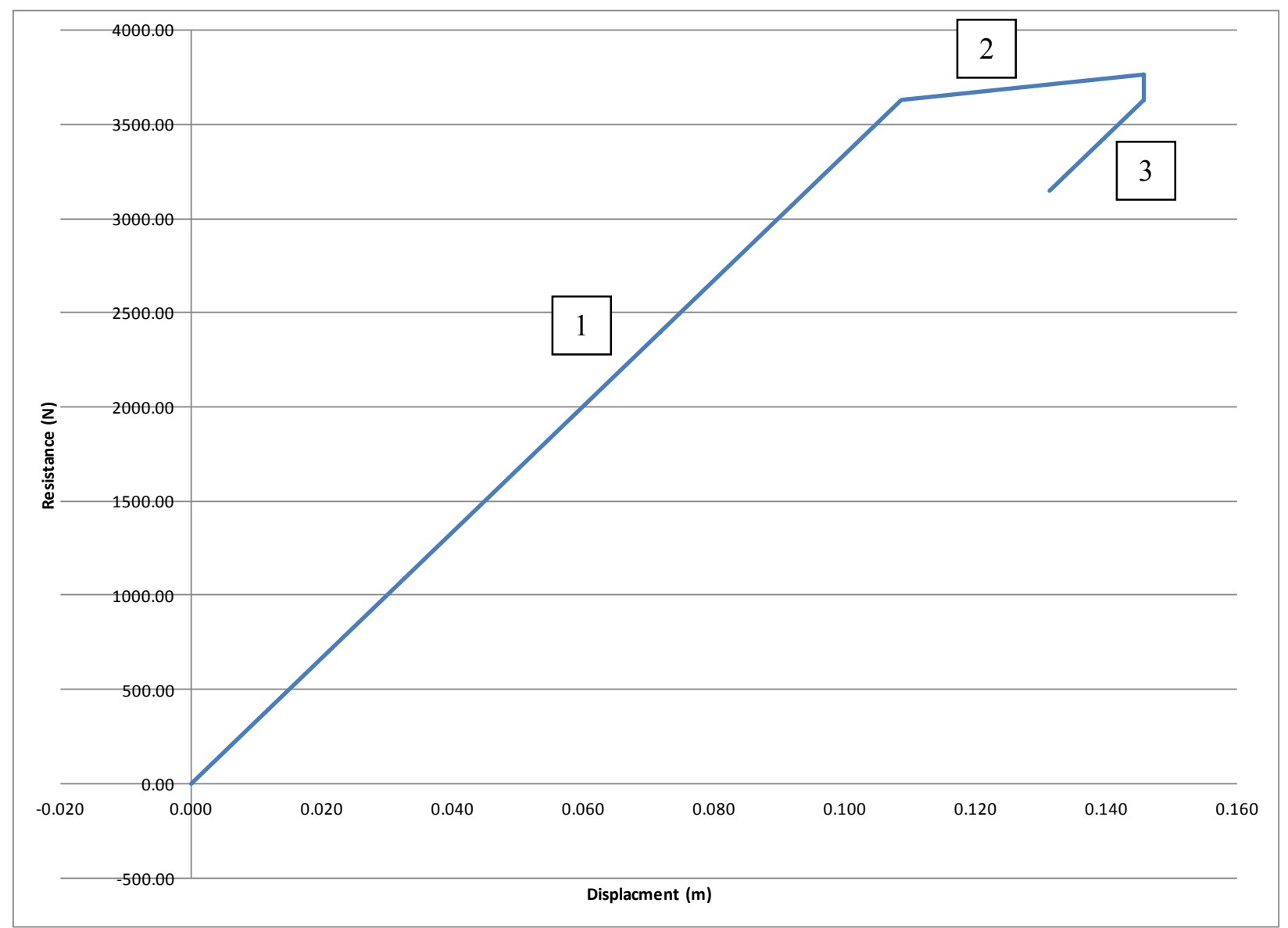

Figure 92: Resistance-deflection curve for steel plate (8 $\mathbf{~ m m ~ t h i c k ) ~}$

Figure 93 and Figure 94 show a comparison between the output from AUTODYN and the developed numerical model for the relation between the displacements and velocities at the mid span with respect to time, respectively. The steel angle deflection exceeded the elastic limit so permanent deformation occurred in the angle as shown in Figure 93. Figure 94 also shows that the velocity of the midspan of the steel angle rested back to zero after the load passed the steel angle and no more movements are taking place. 


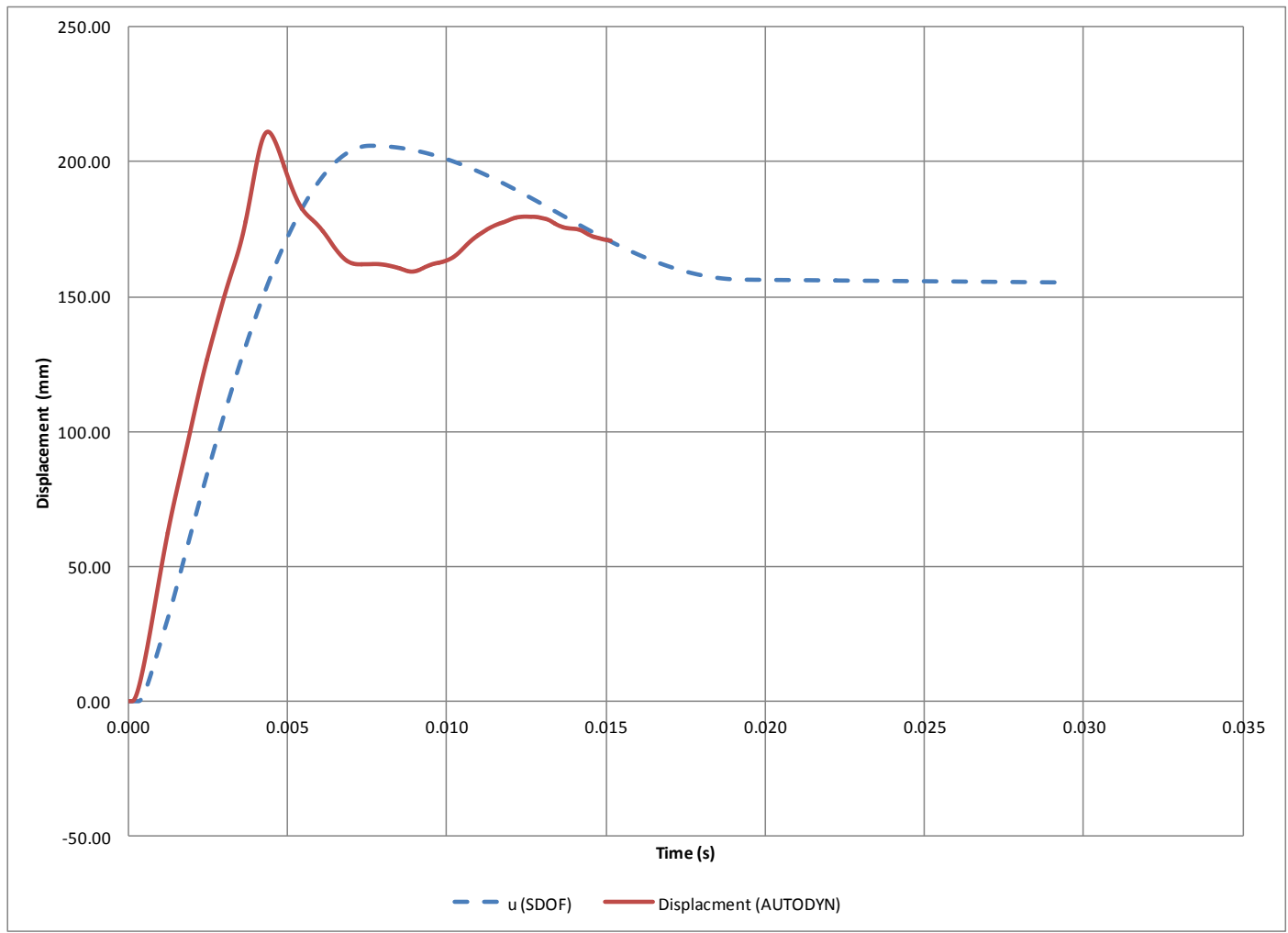

Figure 93: Comparison between obtained results from developed model and AUTODYN to predict displacement at mid span (8 $\mathrm{mm}$ thick)

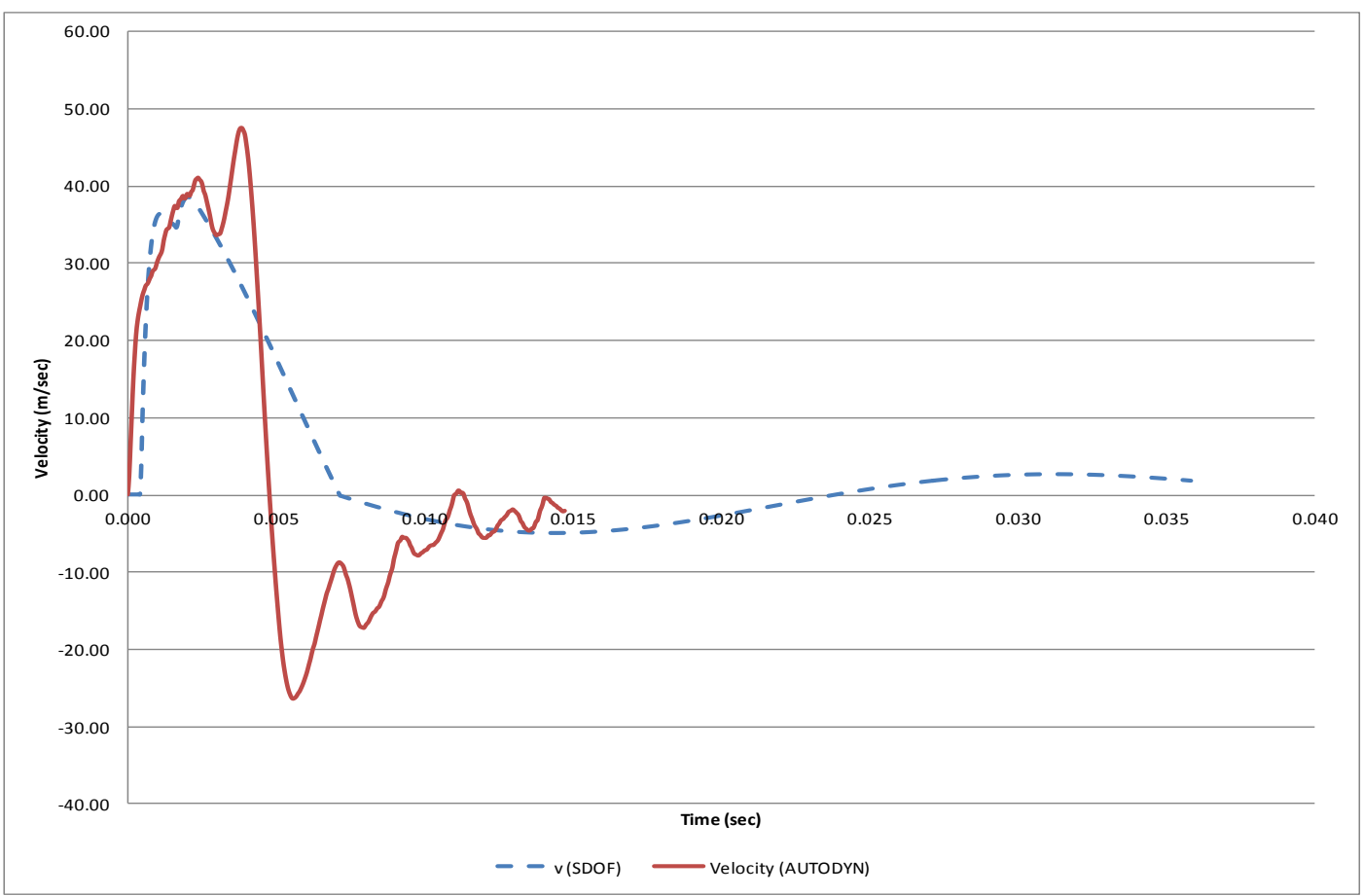

Figure 94: Comparison between obtained results from developed model and AUTODYN to predict velocity at mid span (8 mm thick) 


\subsection{4 $1 \mathrm{~kg}$ TNT charge in a $(2.0 \times 2.0 \mathrm{~m})$ container with 0.5 vent area ratio (Failure occurs)}

The resistance curve of the steel plate with $5 \mathrm{~mm}$ thickness is shown in Figure 95. It can be observed from the figure that the steel plate started to deform elastically until the deflection of the plate exceeded the elastic limit. At this point, tensile membrane action started to take place in the steel plate increasing the resistance of the plate. When the end support rotation exceeds 12 degrees, failure was assumed to take place in the steel plate according the design limits of the UFC3-340-02 (2008).

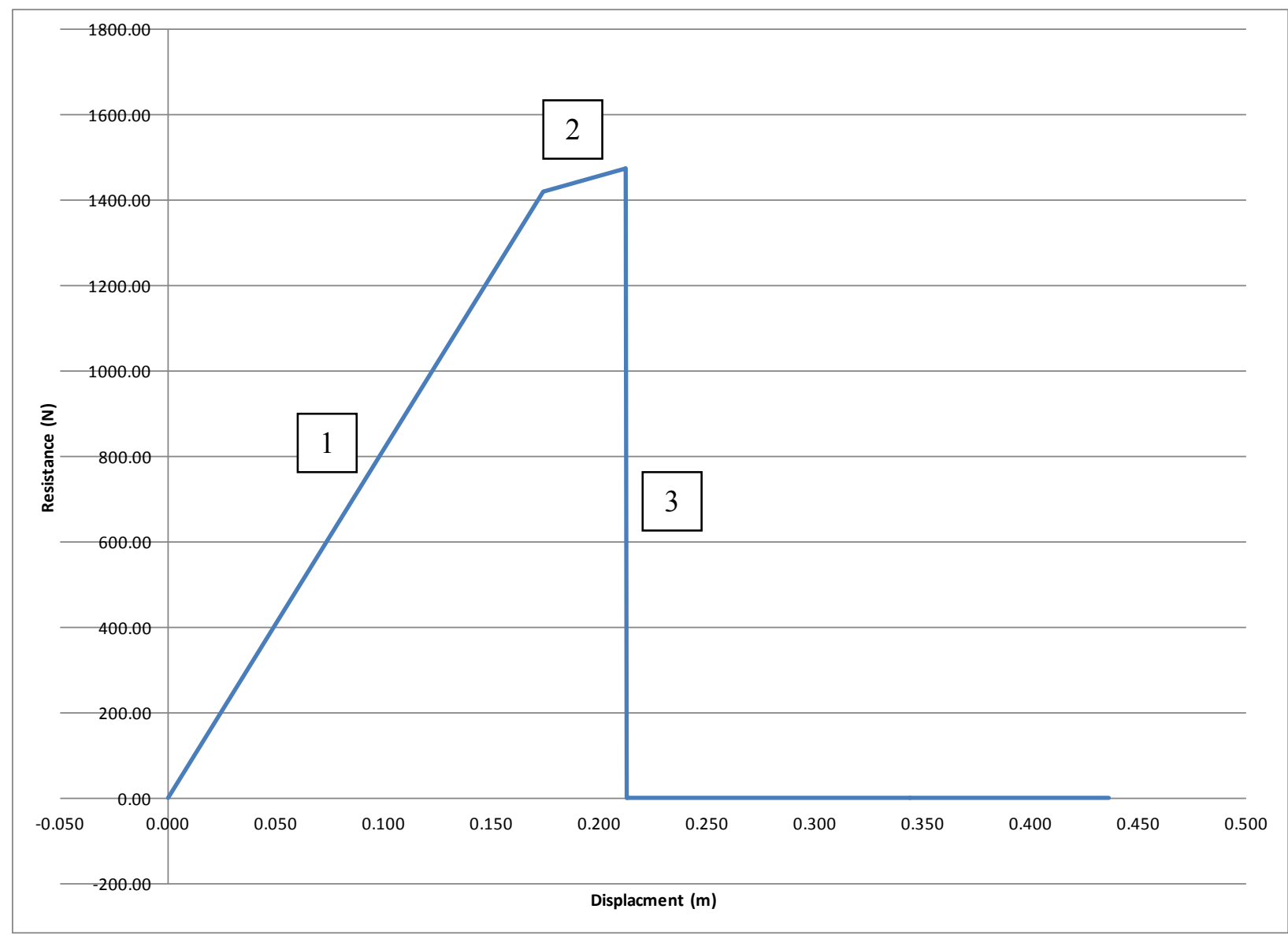

Figure 95: Resistance-deflection curve for steel plate (5 $\mathrm{mm}$ thick)

Figure 96shows a comparison between the output from AUTODYN and the developed numerical model for the relation between the displacements at the mid span with respect to time. In this case the steel plates were not able to withstand the applied load and failure occurred at the mid span of the steel plate. 


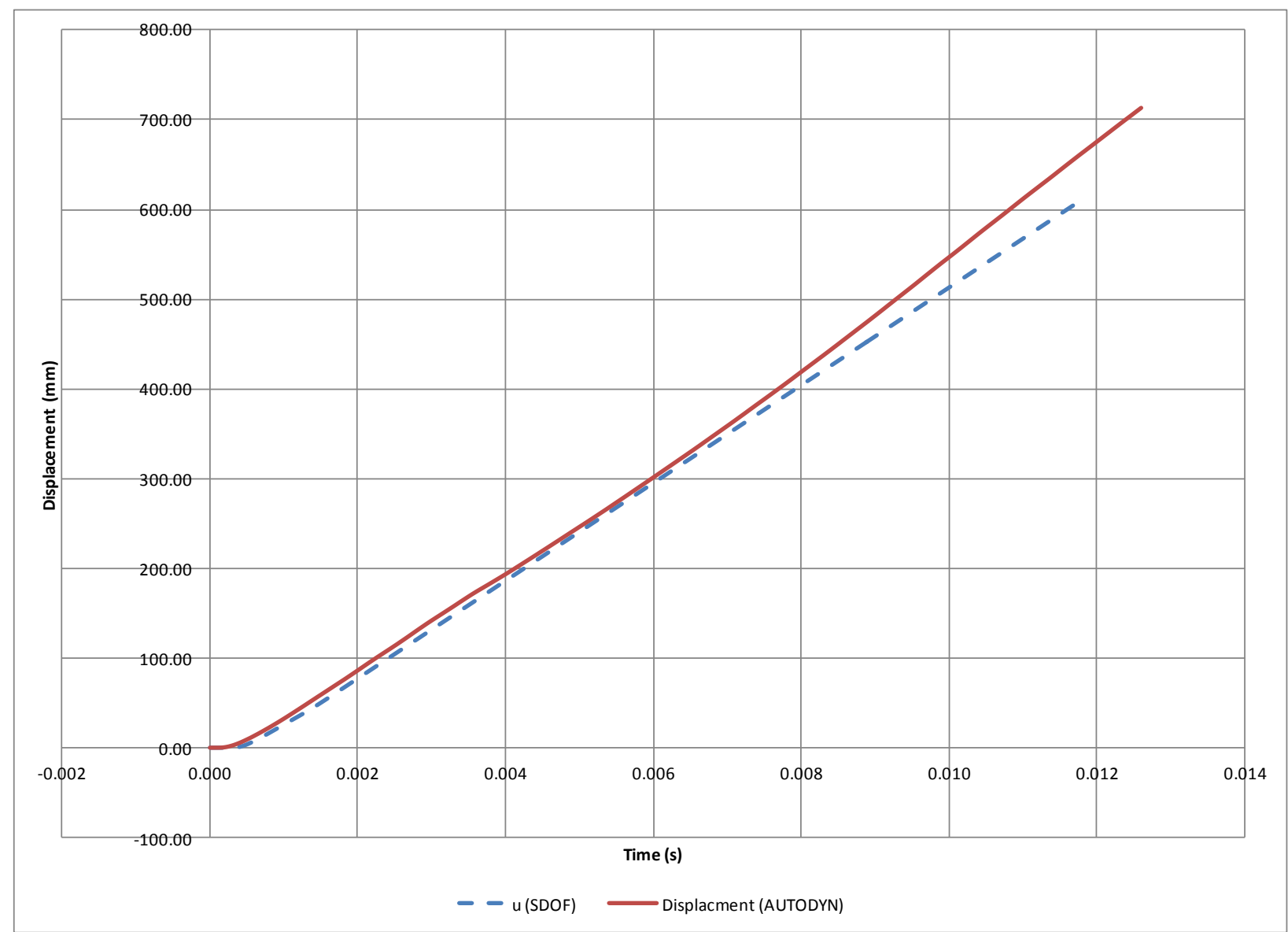

Figure 96: Comparison between obtained results from developed model and AUTODYN to predict displacement at mid span (5 mm thick)

Also, Figure 97 shows a comparison between the output from AUTODYN and the developed numerical model for the relation between the velocities at the mid span with respect to time. It can be seen that the velocity of the gauge at mid span is kept constant. 


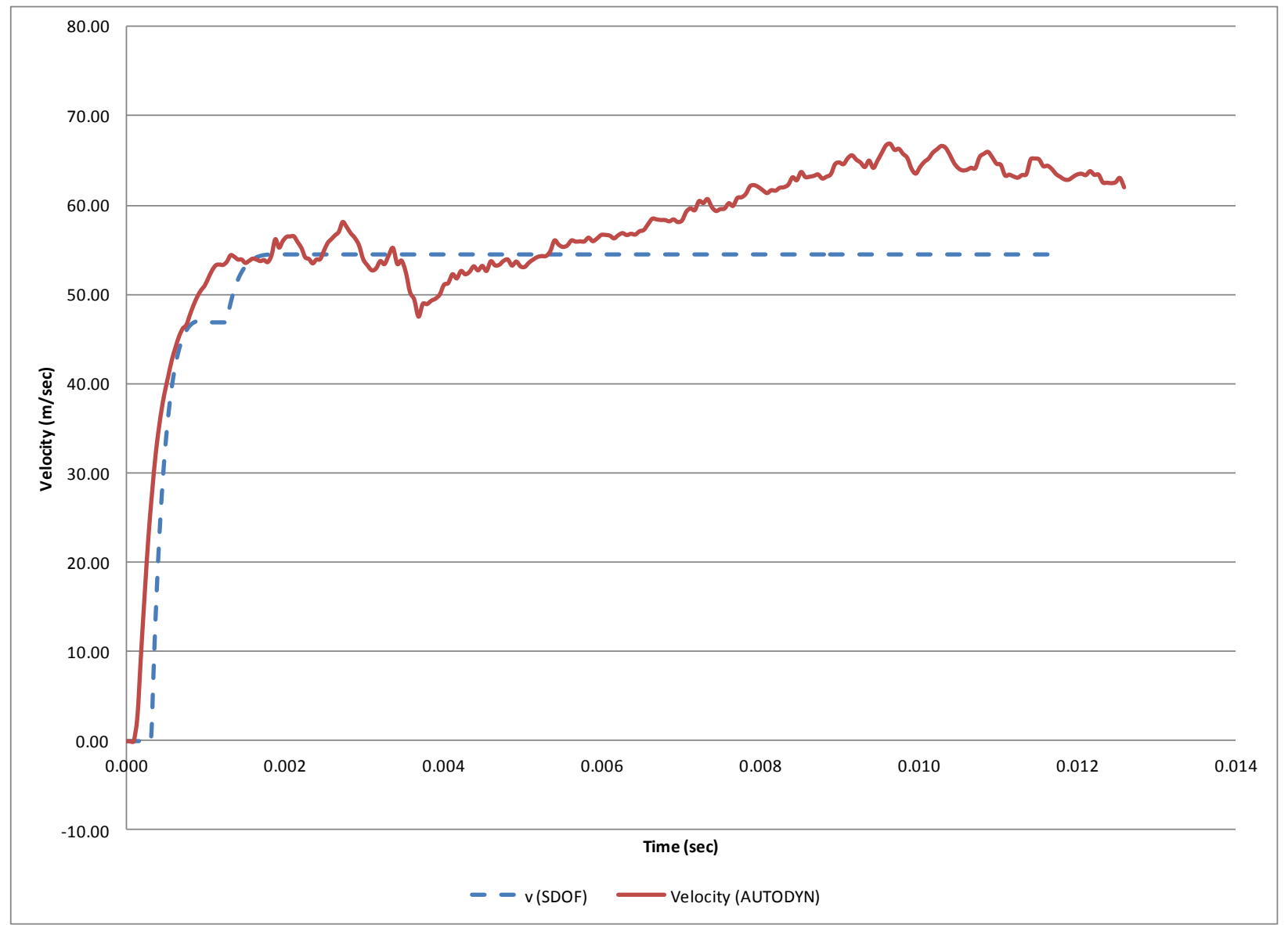

Figure 97: Comparison between obtained results from developed model and AUTODYN to predict velocity at mid span (5 mm thick)

The previous example was to investigate the response of single layer steel plate VSS section due to blast loading. As was illustrated from the results, the resistance of the steel plates mainly depends on their thickness $(t)$. And in order to increase the resistance capacity of the steel plates, the thickness should be increased. However, this will increase the weight of the VSS section and thus the VSS containers won't be economic to transport.

Another way to increase the resistance of the VSS section is to use steel angles with different orientations instead of steel plates. The moment of inertia of steel angles is much higher than that of the steel plate therefore; the required resistance can be achieved using angles without having to increase their thickness. Using steel angles with different orientations and sizes is discussed in the following section. 


\subsubsection{L-shaped steel angles}

This section addresses the use of L-shaped steel angles in the VSS section instead of steel plates. The L-shaped angles used are assumed to be equal leg angle. Using steel angles instead of steel plates will increase the weight of the container; however, the resistance of steel angles is higher than that of the steel plates and would lead to more blast resistant VSS containers. Figure 33 shows the configurations that are studied in this section and compared to the steel plate configuration VSS section (control configuration). The two sections consist of L-shaped side-byside angles but with different orientation with respect to the direction of the blast load as shown in the figure.

The two configurations; Single-layer side-by-side angle exterior-facing and Single-layer side-by-side angle interior-facing were investigated and their response to blast loading compared to that of the single layer steel plate configuration. The vent area ratio for the three sections was kept constant at 0.5 while the size of the container was maintained at $2.0 \times 2.0 \mathrm{~m}$ with a $1 \mathrm{~kg}$ TNT charge detonated at the center of the container. The thickness of the steel elements (plates and angles) was also kept the same and equal to $50 \mathrm{~mm}$. The use of single-layer steel angles in not practical as it will have direct exits for fragments to the exterior, but it was analysed to enable comparison of the performances of single-layer steel plate and steel angle configurations. The models for the three cases were developed in 3D AUTODYN and their deflections at the midspan monitored. Figure 98 shows a comparison between the deflections at mid-span for steel angle compared to the steel plates obtained from AUTODYN. 


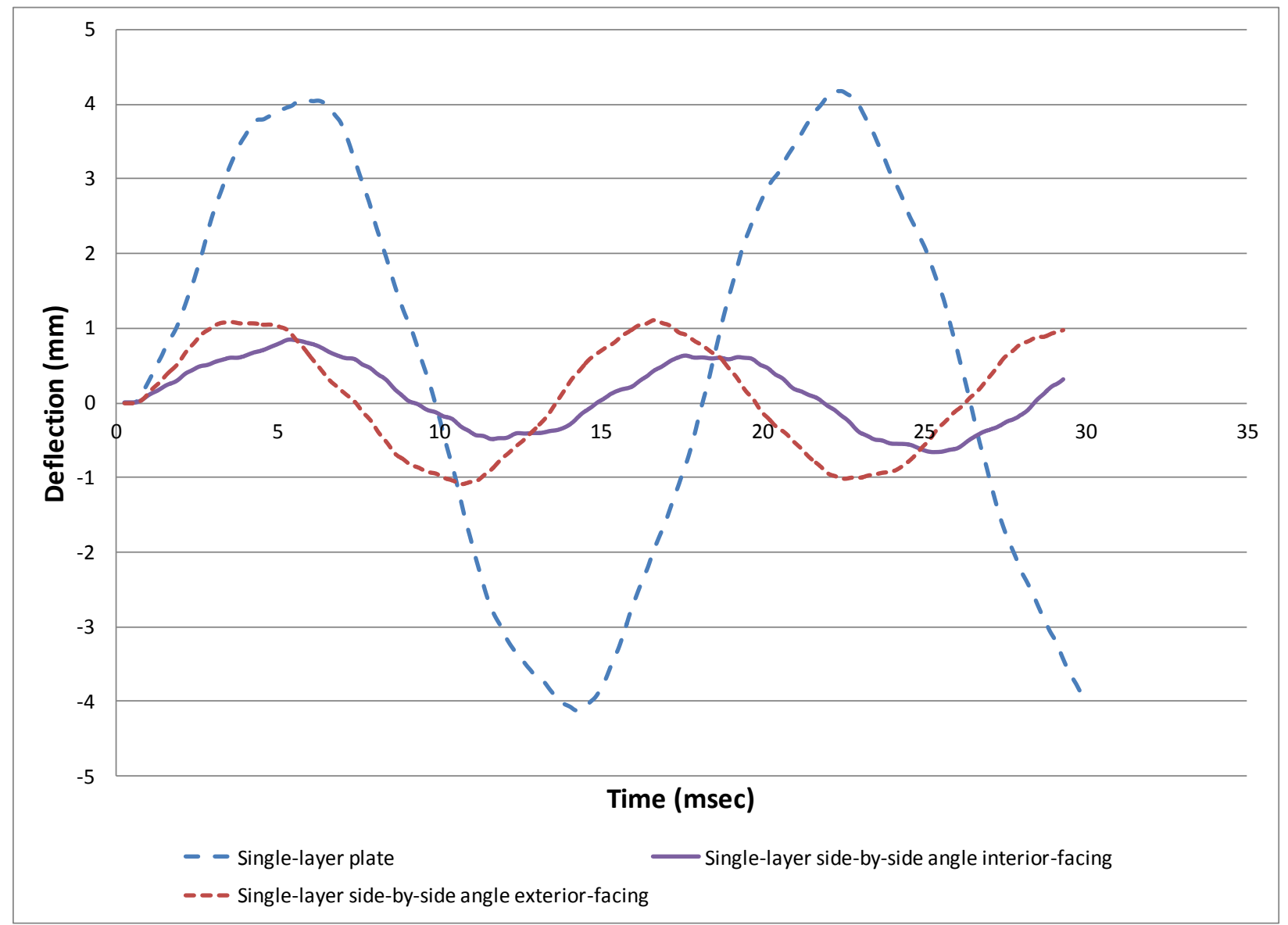

Figure 98: Deflection at mid span for single layer steel plate, Single-layer side-by-side angle exterior-facing, and Single-layer side-by-side angle interior-facing (AUTODYN)

It can be observed from the figure that the steel angles with both orientations responded with lower mid span deflections than the steel plates, as expected. Because the section of the steel angles and plates is $50 \mathrm{~mm}$ thick which is considered to be a thick section, the plates and the angles behaved elastically due to the applied blast load and no permanent deformations were observed. Also, for the Single-layer side-by-side angle exterior-facing, the steel angles experienced more deflection that the steel angles in case of Single-layer side-by-side angle interior-facing although the two sections have the same inertia. This is due to the orientation of the angles with respect to the direction of the blast load. The blast loading on the interior facing angles will tend to act longer in comparison with the exterior facing angles.. 


\subsubsection{Properties of steel angles}

In the previous section, the structural response for steel plates obtained from the SDOF model was addressed. In this section, the structural response for steel angles from the SDOF model will be discussed. For the steel angles, the properties of the section such as the moment of inertia $(I)$ and area $(A)$ were obtained from the of Steel Construction (CAN/CSA S16-01, 2008). The radii of gyration $r_{x}$ and $r_{y}$ were obtained from the section properties as well, but when the steel angle is rotated around its axis, the properties of the section changes. The angle of orientation of the steel angle with respect to the blast load affects the resistance of the section. It is assumed that the steel angles are oriented at 45 degrees as shown in Figure 99.

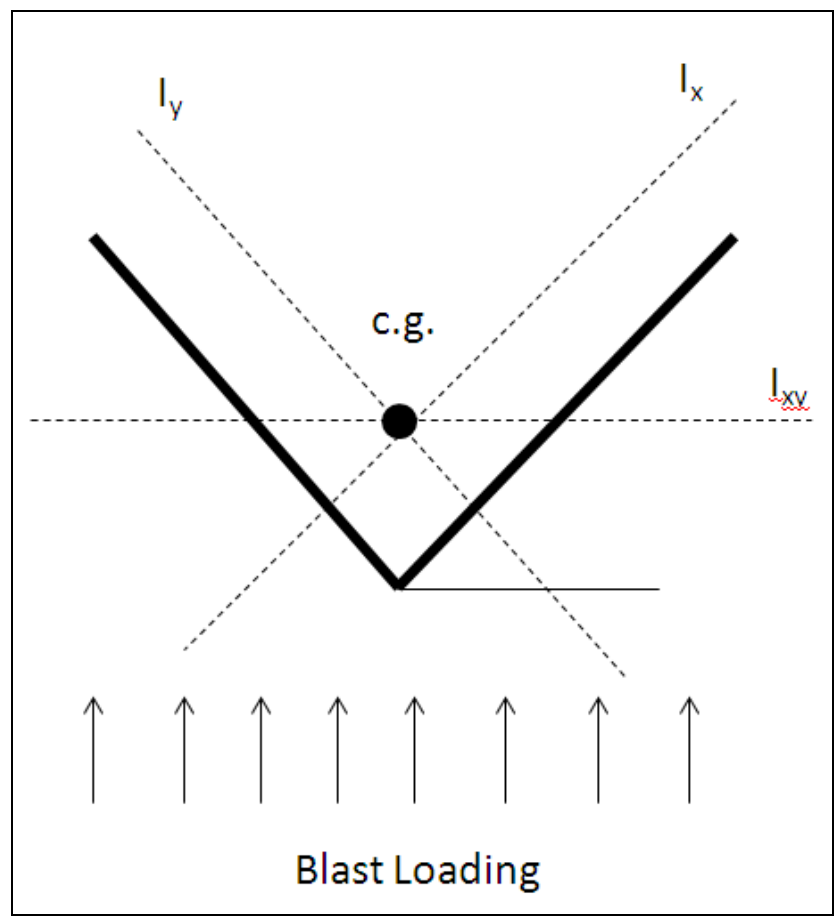

Figure 99: Orientation of steel angles with respect to blast loading (exterior facing configuration)

The moment of inertia of the section for the rotated section is calculated using Equation 44:

$$
I=r_{o}^{2} A
$$

Equation 44

Where $r_{0}$ is the radius of gyration and $A$ is the area of the section. 


\subsection{SDOF analysis of single-layer steel angle configuration}

The response of single-layer steel angle and single layer steel plate configurations to blast loading was addressed in a previous section. In this section, the SDOF method is used to analyse the single-layer steel angle configurations, however the input load is that for a steel plate but with geometric coefficients presented in chapter 5 .

\subsubsection{Single-layer side-by-side angle interior-facing}

The geometric coefficient in this case was found to be 1.70 (See Table 10). This factor is multiplied by the pressure profile obtained from the equations introduced in chapter 4 . To verify the developed model, the following case was studied. The studied case is a $(2.0 \times 2.0 \mathrm{~m}) \mathrm{VSS}$ container with $10 \mathrm{~kg}$ TNT in the center of the container. The VSS section is Single-layer side-byside angle interior-facing with 0.5 vent area ratio. Figure 100 and Figure 101 show a comparison for the output from the developed SDOF model and those obtained from AUTODYN for both velocity and displacement respectively.

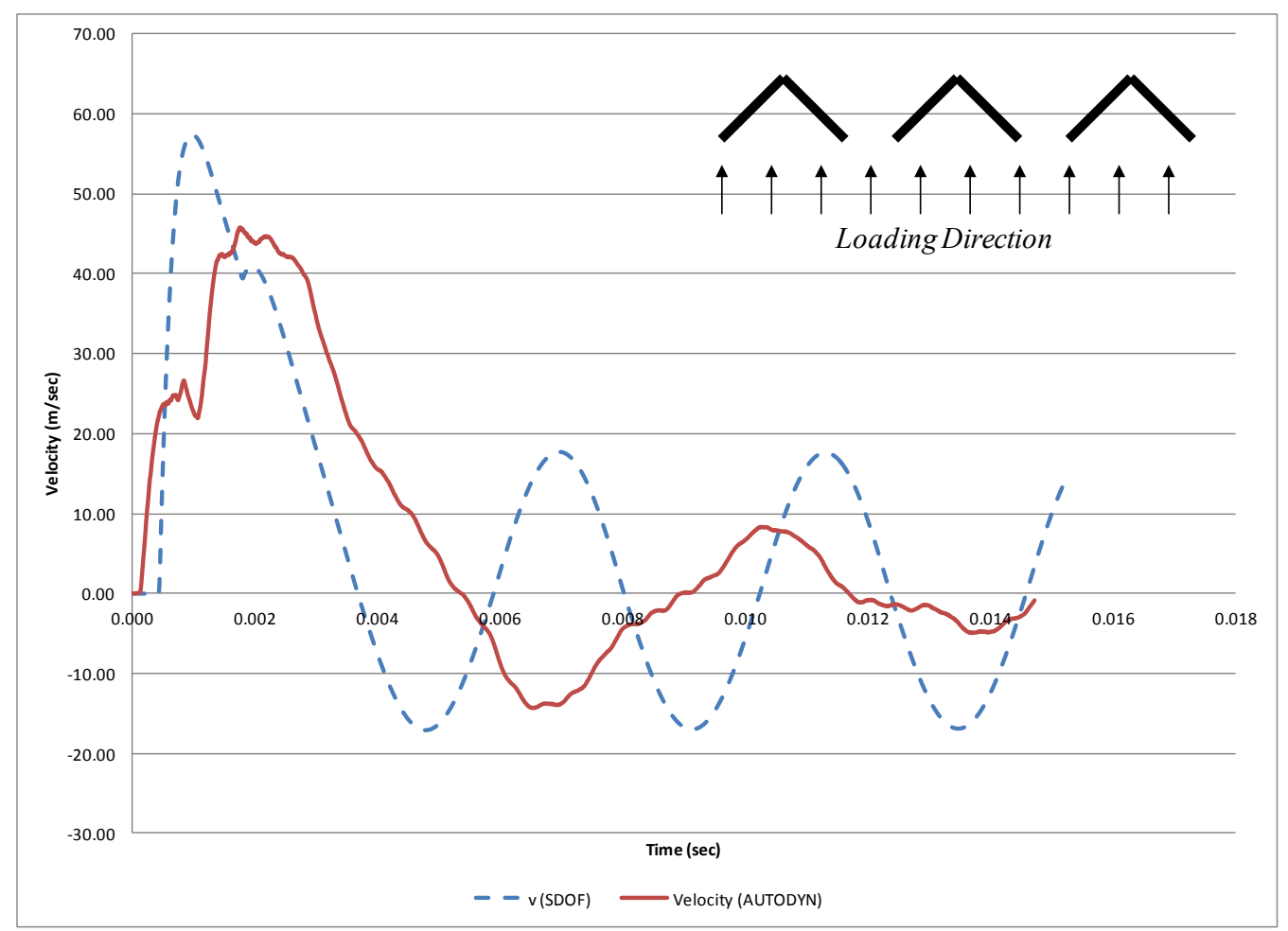

Figure 100: Velocity of steel angles for Single-layer side-by-side angle interior-facing VSS subjected to $10 \mathrm{~kg}$ TNT 


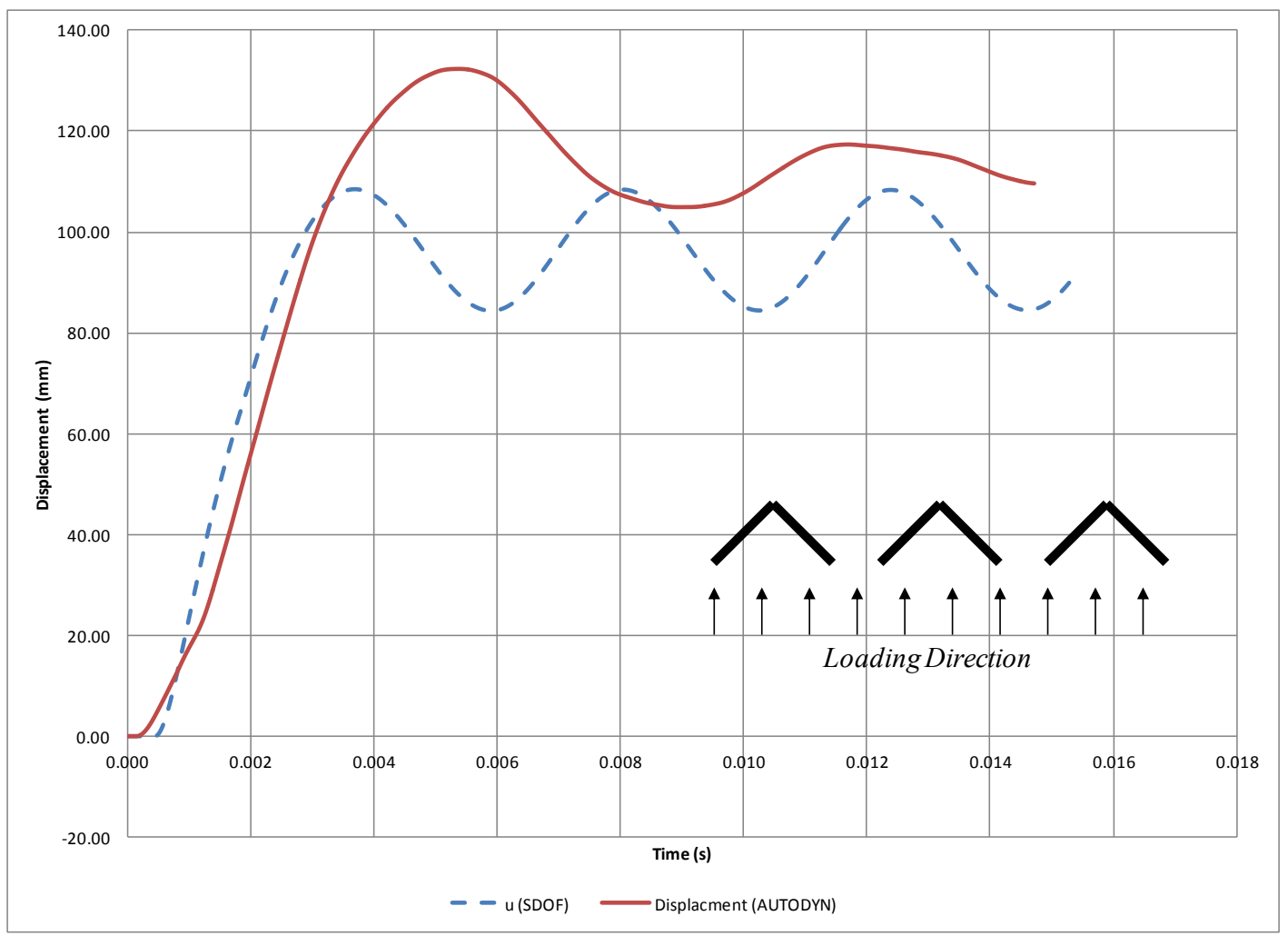

Figure 101: Deflection of steel angles for Single-layer side-by-side angle interior-facing VSS subjected to 10 kg TNT

It should be noted that the damping ratio in the SDOF model was taken equal to zero. This explains that the vibration of the steel beam does not decay to zero as in the case of the AUTODYN model that includes damping ratio in the calculations. Additionally, The SDOF was able to predict the stiffness of the angles and the maximum deflection. However after obtaining the maximum displacement, the natural period of vibration obtained from the SDOF wasn't the same as that obtained from AUTODYN. This is due to the deformation that occurred in the steel angles that changed their geometry from an angle to an irregular shape that affects its stiffness and thus affecting the properties of the section. These changes were not taken into account in the case of SDOF and that is the reason why the two curves does not match in the zone past the maximum deflection. 


\subsubsection{Single-layer side-by-side angle exterior-facing}

The same procedure was carried out for the case of the Single-layer side-by-side angle exterior-facing. For this configuration, the geometric coefficient that has to be multiplied by the modified Friedlander equation was 0.50 . The same comparison done in the previous case is presented in this section but for the Single-layer side-by-side angle exterior-facing. Figure 102 and Figure 103 show a comparison between the SDOF output and the AUTODYN output for both velocity and deflection, respectively. These results are for a 2.0×2.0 m Single-layer side-byside angle exterior-facing with 0.5 vent area ratio and with $1 \mathrm{~kg}$ of TNT in the center of the container. 


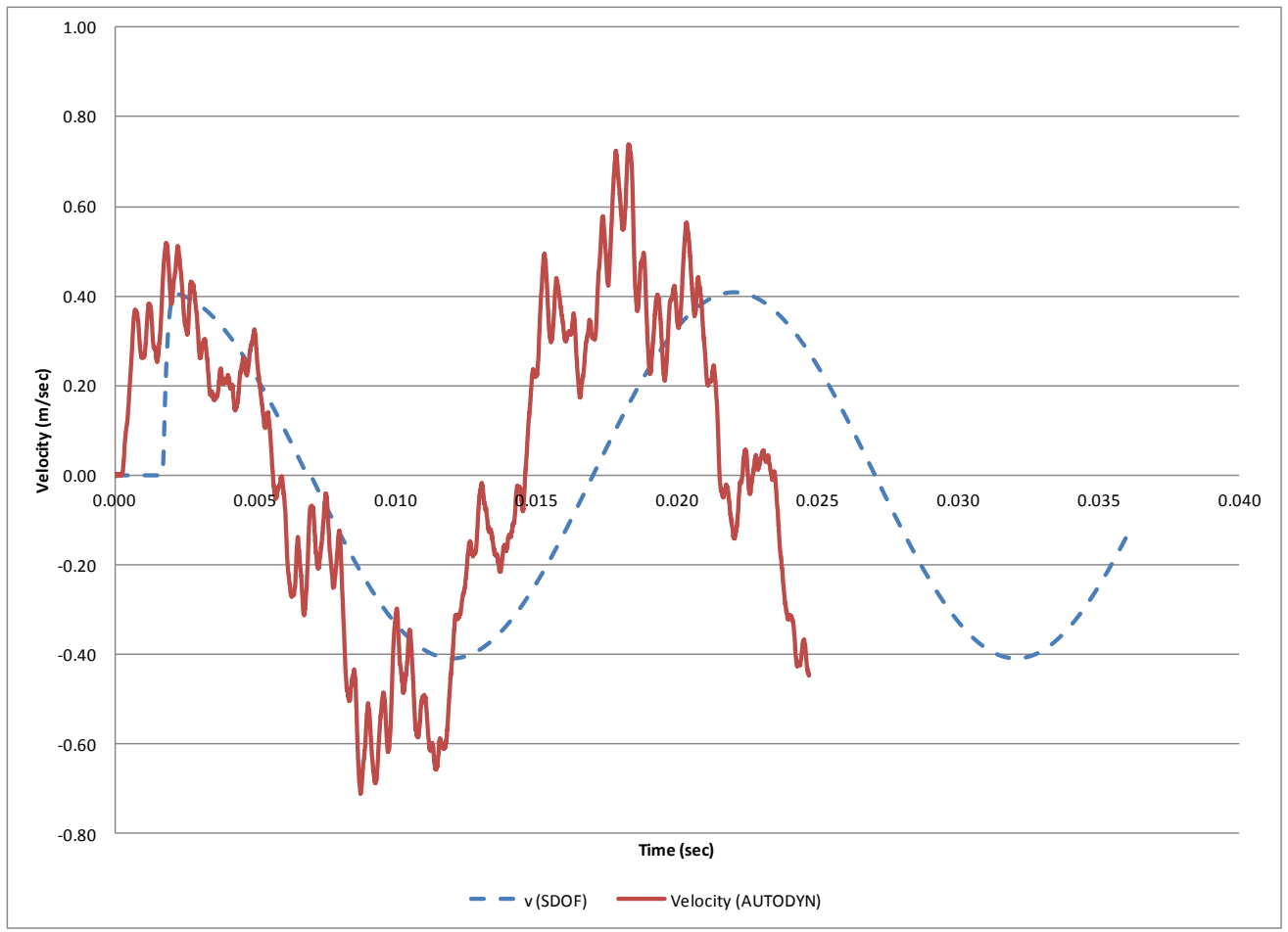

Figure 102: Velocity of steel angles for Single-layer side-by-side angle exterior-facing VSS subjected to $1 \mathrm{~kg}$ TNT

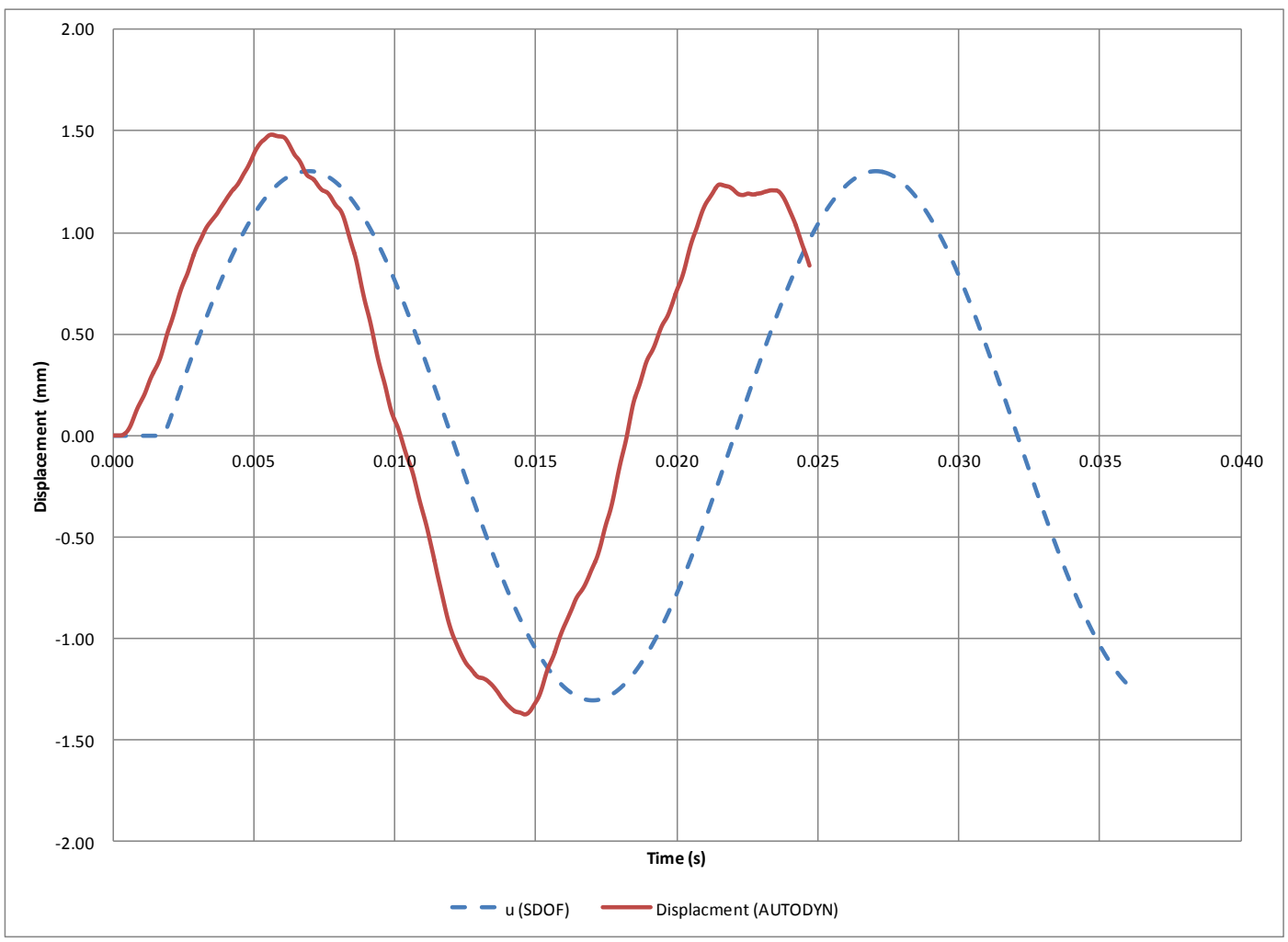

Figure 103: Deflection of steel angles for Single-layer side-by-side angle exterior-facing VSS subjected to $1 \mathrm{~kg}$ TNT 


\subsubsection{Double-Layer Plate Configuration}

The double layer plate configuration consists of two single layer plate configuration arranged in a staggered fashion as shown in Figure 104. The distance between the two layers of plates is indicated as $g$ and it was chosen to be half to the spacing between the plates in one of the layers $a$. The effective vent area ratio $\left(\alpha_{e f f}\right)$ for this section is 0.25 as was calculated by previous equations mentioned in Chapter 3.

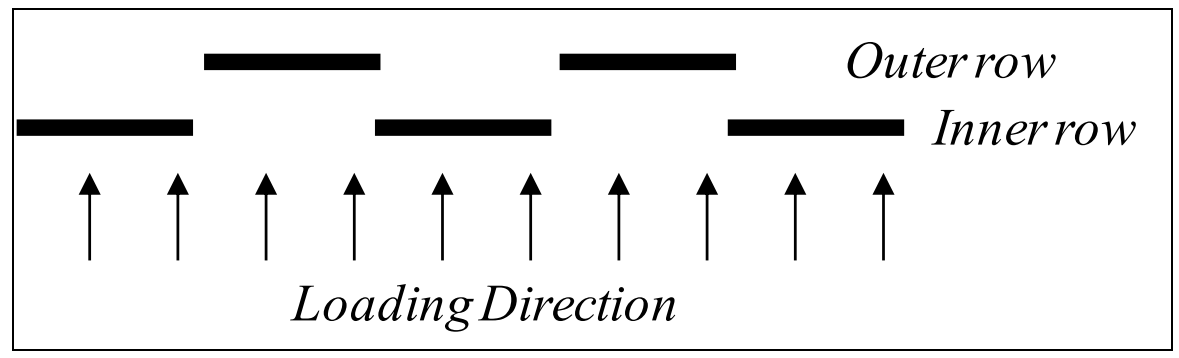

Figure 104: Double-layer plate configuration

The deflections of the plates were recorded for both the inner row and the outer row of plates for a $2 \times 2 \mathrm{~m}$ container with an internal charge equals to $20 \mathrm{~kg}$ TNT charge. Figure 105 shows the deflection of the steel plates for both rows obtained from AUTODYN and those obtained from the SDOF model. The curve shows that the outer row of plates experience larger deflection than those of the inner row plates. Although the steel plates are of the same size and orientation with respect to the blast wave, This is due to the jetting effect resulted the blast wave passing through the inner row of plates that increased the load on the outside row of plates. This increase in the load increased the deflection of the outer row of plates compared to those of the inner row. The figure also shows a good correlation between then deflections obtained from the SDOF model and those obtained from AUTODYN. 


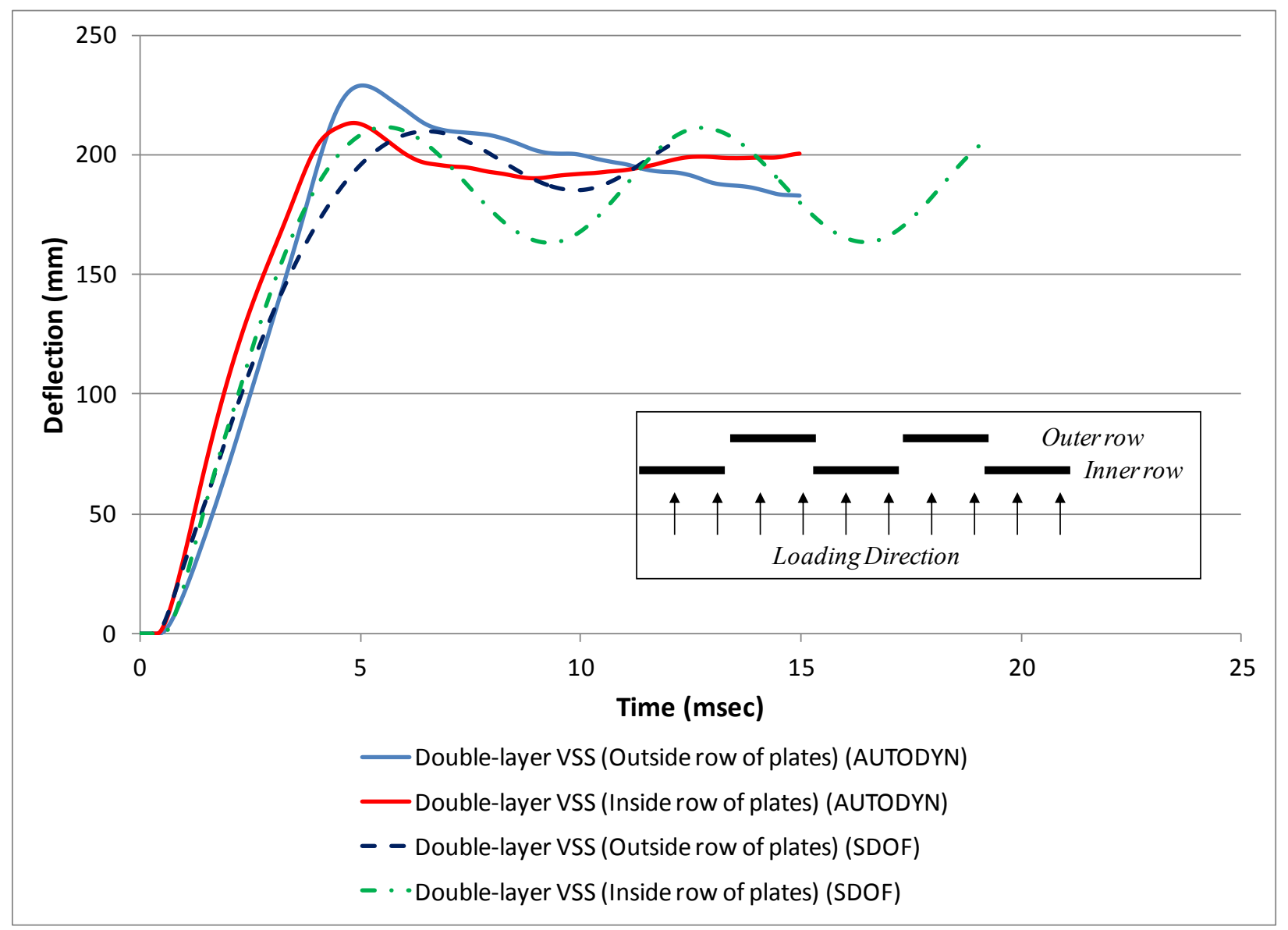

Figure 105: Deflection of steel plates in a Double-layer plate configuration

\subsubsection{Double-Layer Nested Side-By-Side Angle Configuration}

In Chapter 4, it was found that the Double-Layer Nested Side-By-Side Angle configuration had the best level of attenuation compared to the other studied VSS configurations. In this section, the structural response of the angles forming this configuration is studied. The studied case is the same one studied in the previous section for the Double layer plate configuration but with changing the geometric configuration. Figure 106 shows the Double-layer nested side-by-side angle section that was used to study the performance of this configuration. The effective vent area ratio for this section was 0.25 . The deflection of the steel angles was studied for both the inner and outer layer of steel angles forming the VSS section. Figure 107 shows the deflection of the steel angles in a Double-layer nested side-by-side angle configuration. 


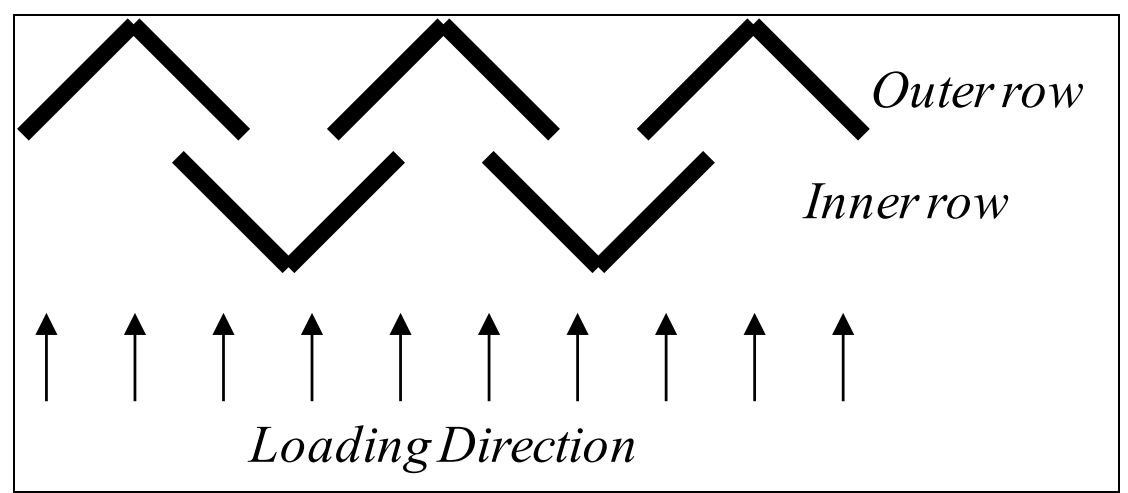

Figure 106: Double-layer nested side-by-side angle configuration

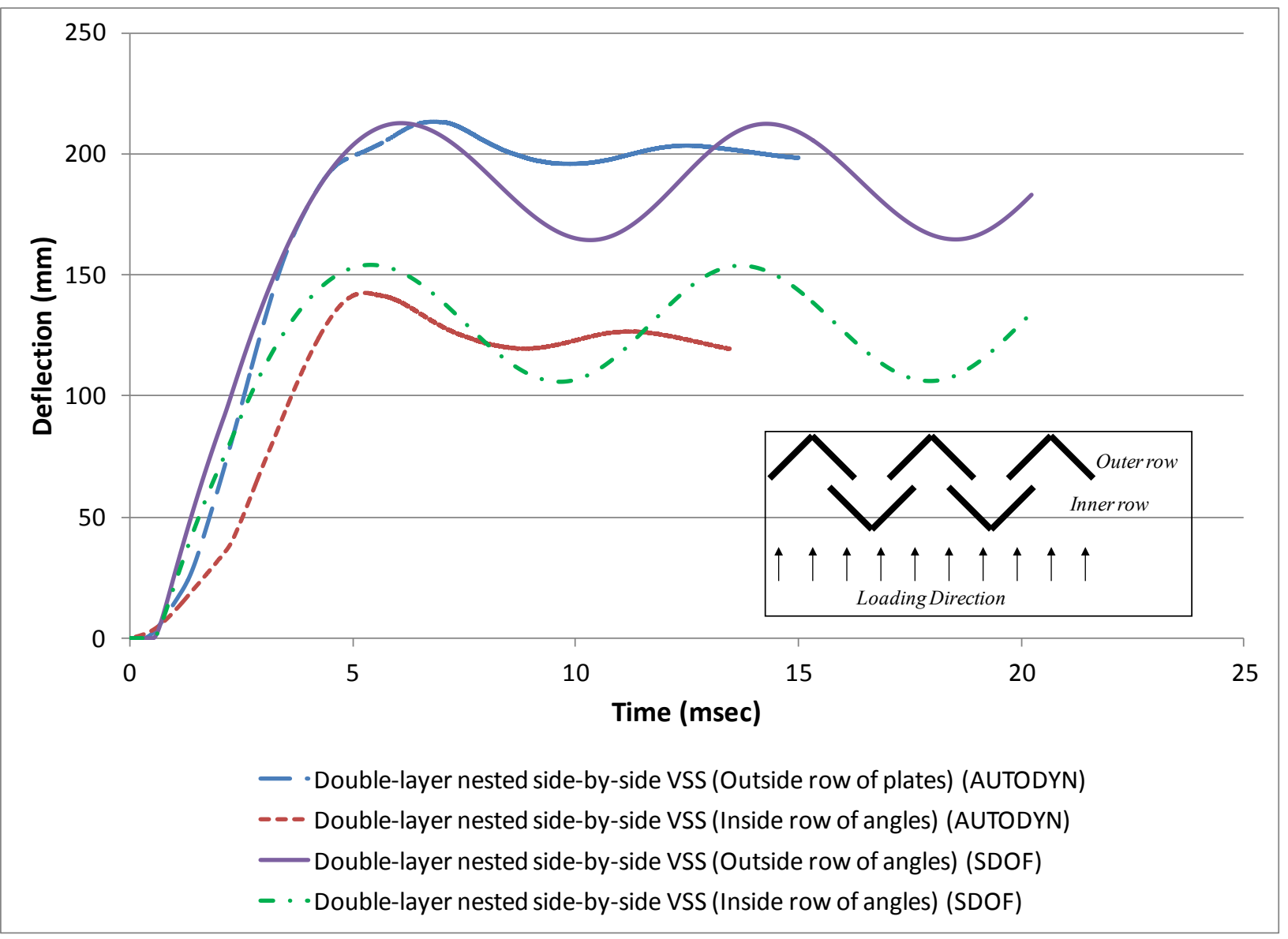

Figure 107: Deflection of steel angles in a Double-layer nested side-by-side angle configuration (AUTODYN) 
Figure 107 shows that the deflection of the outer row of steel angles experienced more deflection that the inner row of angles. The main reason behind this is that the geometry of the outer row of steel angles increases the duration of the load applied on the steel angle as was discussed before in Chapter 5. The other reason is again because of the extra load obtained from the wave jets from the first layer as in the case of the Double layer plate configuration (inner row).

Another observation that should be stated here is that the deflection of the outer row angles was almost the same as the deflection of the plates in case of the double layer plate configuration in spite of the higher resistance of the angles compared to that of the plates. This is because of the change of the pressure profile on the angles due to their geometric shape.

\subsection{Tension membrane}

Calculating the tensile membrane force was calculated by assuming that the steel member experience a vertical elongation due to the large deflection of the member. The elongation in the member is directly proportional to the lateral deflection of the steel beam as shown in Figure 108 below.

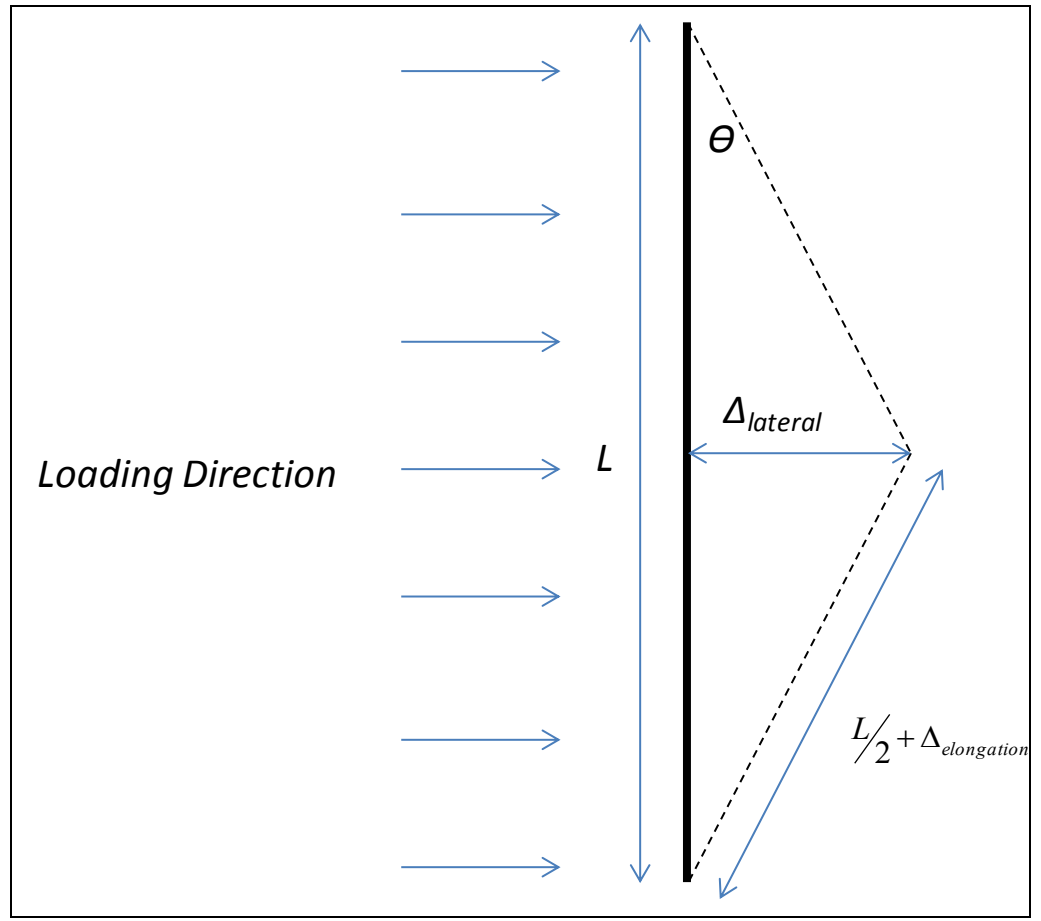

Figure 108: Deflection of the steel plates (angles) 
The Length $(L)$ is the original length of the steel plate or angle, $\left(L^{\prime}\right)$ presents the length of the steel plate after deformation and the $\Delta_{\text {lateral }}$ presents the lateral deflection of the member due to the applied load. The elongation in the steel plate can be calculated by the equation below:

$$
\left(\Delta_{\text {elongation }}\right)=\frac{(L / 2)}{\cos \theta}-(L / 2)=(L / 2)\left(\frac{1}{\cos \theta}-1\right)
$$

\section{Equation 45}

Where $\theta$ is the angle of rotation at the end supports of the steel plate and it is directly proportional with the lateral deflection $\left(\Delta_{\text {lateral }}\right)$. Assuming that the steel plate is under pure tension, the equation below is used to calculate the tension force in the member depending on the elongation that took place.

$$
T_{\text {membrane }}=\frac{\Delta_{\text {elongation }} E A}{L}
$$

Equation 46

Figure 109 shows the SDOF model output for the axial force versus time for the steel plate for different plate thickness; 50, 10,8 and $5 \mathrm{~mm}$ thick. The length of the steel plate is 2000 $\mathrm{mm}$ while the width is $200 \mathrm{~mm}$. The plate was subjected to an internal explosion of $1 \mathrm{~kg}$ TNT. The graph shows the effect of the tensile membrane action on the steel plate. 


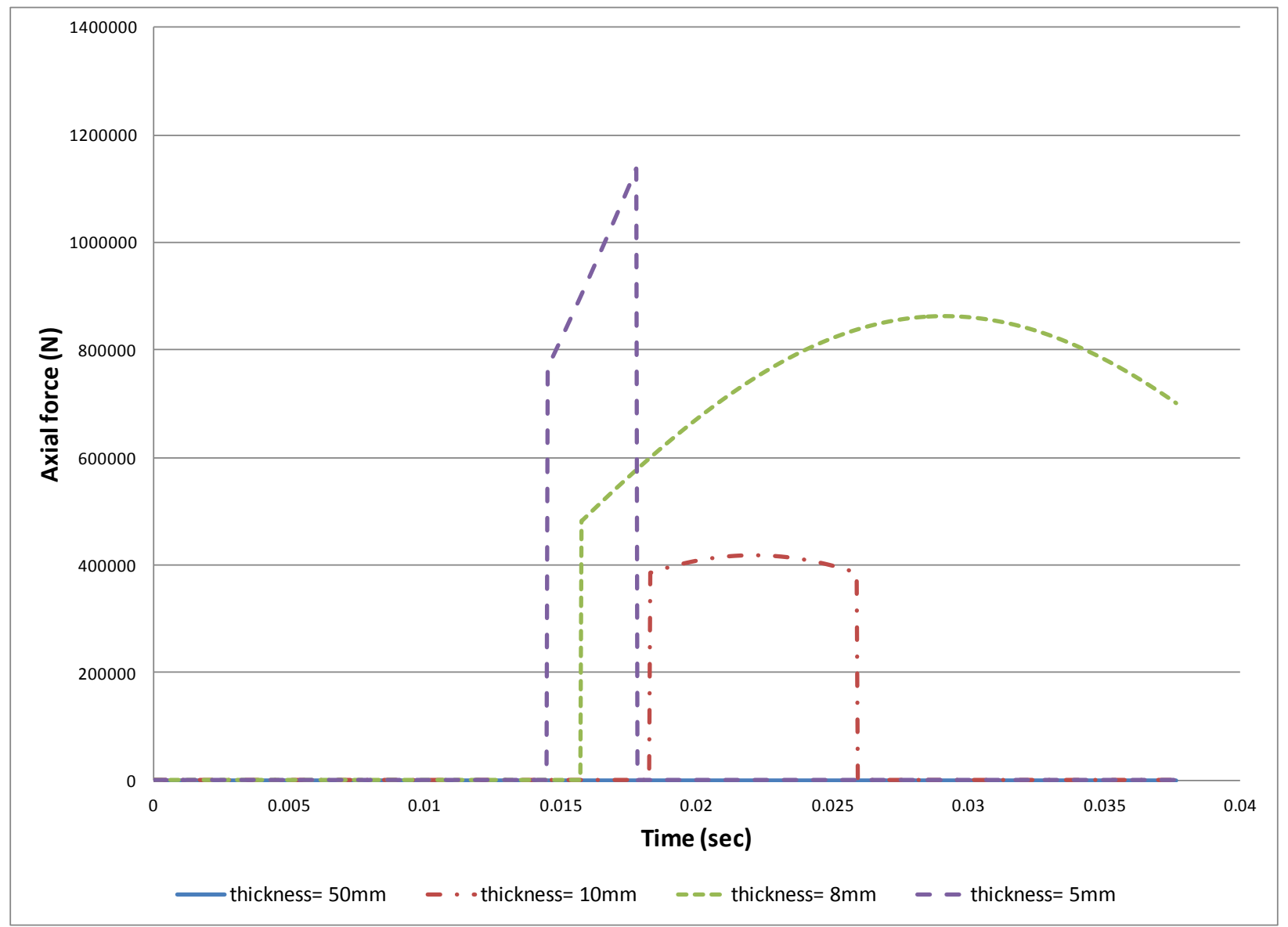

Figure 109: Tensile membrane force VS time

It can be seen from the figure that no membrane action took place in case of the steel plate equal $50 \mathrm{~mm}$. This is because the deflection of the steel plate was less than the elastic deflection of the plate and therefore, no plasticity took place. Reducing the thickness to $10 \mathrm{~mm}$ transferred the behaviour of the steel beam to plastic. Tensile membrane action took place in the steel plate but when the load passes the member and the member is unloaded, the tension force was reduced back to zero. Same phenomenon was observed for the $8 \mathrm{~mm}$ thick plate, however, in that case, the tensile force still applied on the steel plate because the deformation in the steel plate was permanent.

Reducing the thickness more than that leads to failure in the steel plate, so it can be seen from the figure that in case of the $5 \mathrm{~mm}$ thick steel plate, the tensile force kept increasing until failure occurs and then drops to zero. 


\subsection{Welding}

After studying the failure of the steel members of the VSS, it was found that the failure was due to the rotation at the end supports. Assuming that the steel angles of the side wall of the VSS section are fixed at both ends, it is expected to have reaction forces (shear and tension) as well as moment at the end supports. These reactions at the supports should be resisted by means of the connection between the steel angles forming the side wall of the VSS section and the main frame of the container. The connection between the steel members of the side wall of the container and the main frame of the container is assumed to be welding process. The welding connection should be able to withstand the forces at these connections. The goal of the design process is to determine the required type of welding and welding size needed to resist these reactions.

Support shears or reactions are a function the applied load and the maximum resistance attained by the structural element. However, for short duration blast loads, the support shears can be reasonably estimated by neglecting the applied load. The shear force and the moment were obtained using the equivalent static loads and these forces were used for the design or analysis of weld connections as per UFC3-340-02, (2008). In this approach, the ultimate flexural resistance of the component was applied as an equivalent static load to determine the maximum end reaction loads on the connections. The ultimate dynamic capacity of the connection should be equal to, or greater than the equivalent static reaction load. The ultimate dynamic connection capacity can be based on Load and Resistance Factor Design (LRFD), using the applicable dynamic yield strength of the connection material in place of the static yield strength. The applicable strength reduction factor (i.e., the $\phi$ factor) from LRFD design should typically be included in the dynamic connection strength. According to UFC3-340-02, (2008), the DIF for structural bolts and welds, which typically have high yield strength greater than approximately $620 \mathrm{MPa}(90,000 \mathrm{psi})$, is only on the order of 1.05. The following equations are used to predict the shear forces and the moments at the supports. 


$$
\begin{array}{r}
r_{u}=\frac{384 \Delta_{e} * E^{*} I}{5 L^{4}} \\
V_{s}=\frac{r_{u} * L}{2} \\
M_{u l t}=\frac{r_{u} * L^{2}}{12}
\end{array}
$$

Equation 48

Equation 49

(N.mm)

\section{Equation 47}

Where $r u$, is the equivalent static load in $\mathrm{N} / \mathrm{mm}$

Also, when the steel angles are overloaded by blast loads, their structural response often transform from flexural response to tension membrane, or catenary response. The tension reactions at the end supports should be taken into consideration. The tension force depends on the vertical displacement elongation that is experienced in the steel plate. The vertical elongation is given by the Equation 50:

$$
\Delta_{\text {elongation }}=L(\cos \theta-1) \quad(\mathrm{mm})
$$

\section{Equation 50}

Where $\theta$ is the angle of rotation at the end support. It can be seen from the elongation in the steel element depends on the end support rotations. However, this elongation occurs when the deflection of the steel plate exceeds the thickness of the plate.

After obtaining the elongation that is experienced in the steel plate, the axial force is computed by the Equation 51. The first term is due to the deformation that occurred in the steel plate while the second term is due to the effect of the moment that exerts additional tension force on one of the sides of the weld. 


$$
T=\frac{\Delta_{\text {elongation }} * E^{*} A}{L}+\frac{M}{t / 2}
$$

Equation 51

The kind of weld connecting the steel plates and the main frame of the container is the fillet weld as shown in Figure 110. The minimum size of the fillet weld that can be used is $3 / 4$ the plate thickness. This is to develop full plate strength (Blodgett, 2002). However, the size of the fillet weld is assumed to be equal to the thickness of the steel plate $t$ based on standard allowable. Fillet weld is supposed to resist both tension and shear forces. The resistance of the fillet to transverse load (shear force) depends on the length of the weld parallel to the direction of the load. While for the axial load, it is resisted by whole weld length around the steel plate.

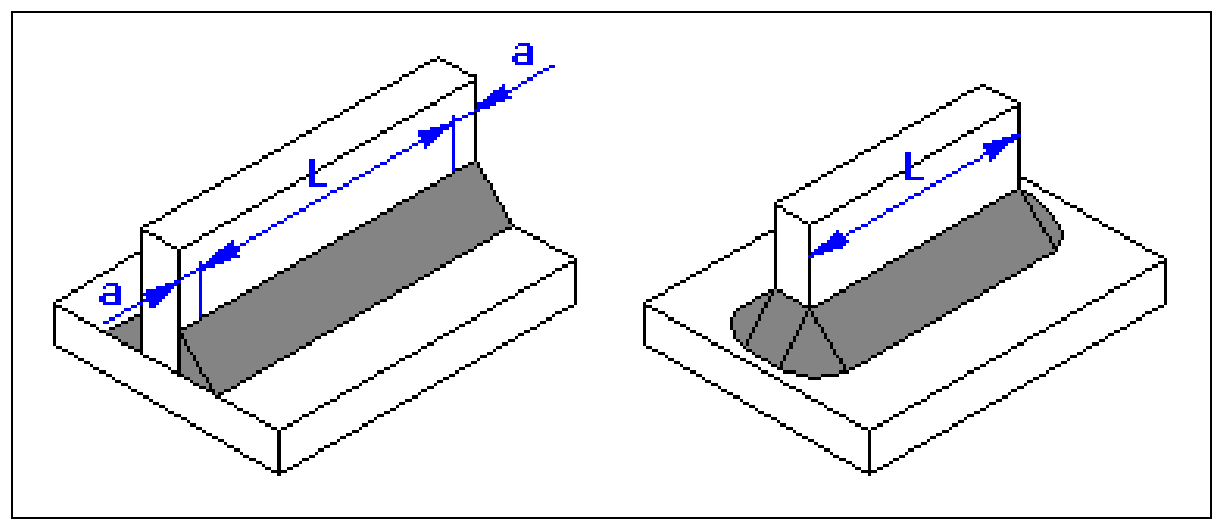

Figure 110: Fillet weld

Where $a$ is the fillet weld throat thickness defined as the height of the biggest isosceles triangle inscribed into a weld section without penetration. The value $a$ depends on the weld size. While $L$ is the length of the weld and it depends on the length of the member.

Fillet welds are most common and used in all structures. Weld sizes are specified in 1/16 inch increments. A fillet weld can be loaded in any direction in shear, compression, or tension. However, it always fails in shear. The shear failure of the fillet weld occurs along a plane through the throat of the weld, as shown in the Figure below. 


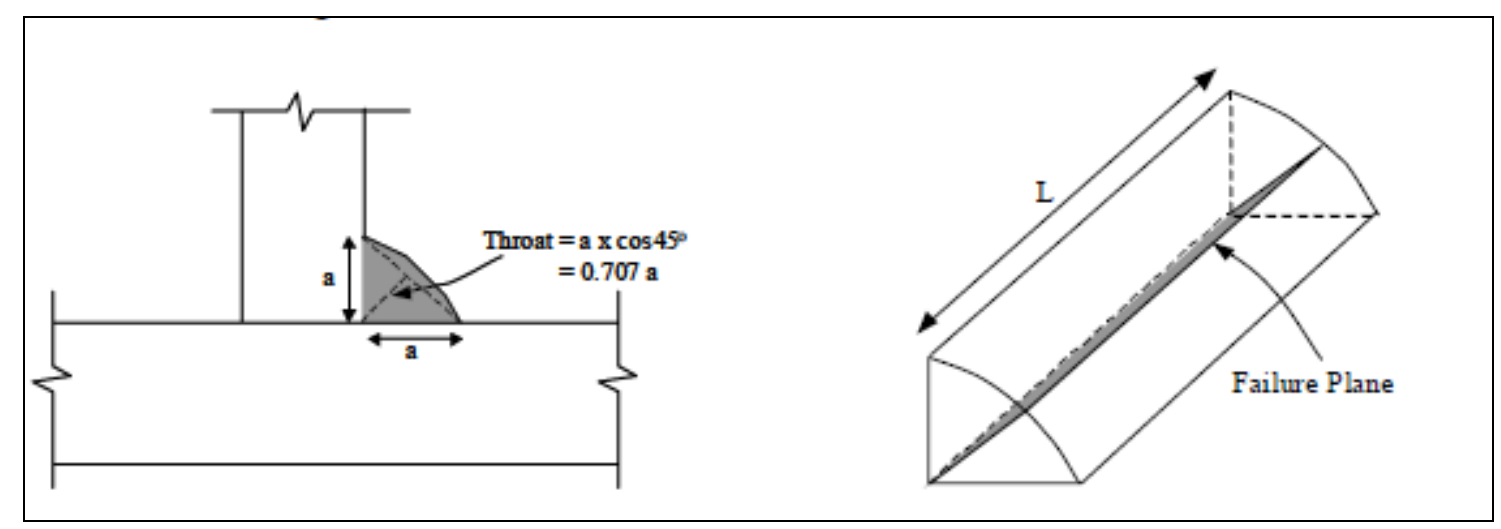

Figure 111: Failure plane for fillet weld due to shear force

It is strongly recommended to check weld metal and base metal strength. Smaller value governs. In most cases, the weld metal strength will govern. The equations used for the design of the weld connection for both the weld and base metal are shown in Equation 52 below:

$$
V_{r}=0.67 \phi_{w} A_{w} X_{u}\left(1+0.5 \sin ^{1.5} \theta\right) \quad \text { for the weld metal }
$$

\section{Equation 52}

Where $A_{w}$ is the area of weld $\left(\mathrm{mm}^{2}\right)$ and $X_{u}$ is the ultimate strength of the weld (MPa). Conservatively, the term $\left(1+0.5 \sin ^{1.5} \theta\right)$ is taken equals to 1 as mentioned in the Handbook of Steel Construction (CAN/CSA S16-01, 2004). Additionally, the shear strength of the base metal must also be considered (Equation 53):

$$
V_{r}=0.67 \phi_{w} A_{m} F_{u} \quad \text { for the base metal }
$$

Equation 53

Where $A_{m}$ is the shear area of effective fusion face $\left(\mathrm{mm}^{2}\right)$ and $F_{u}$ is the tensile strength of the welded element (MPa). Another factor that needs to be taken into consideration is the angular torsion that occurs on the thin plate due to the applied load.

The tension resistance of the weld is given by the Equation 54 below (Handbook of Steel Construction (CAN/CSA S16-01, 2008)):

$$
T_{r}=\phi_{w} \sqrt{\left(A_{n} F_{u}\right)^{2}+\left(A_{w} X_{u}\right)^{2}} \leq \phi A_{g} F_{y}
$$

\section{Equation 54}

Where $A_{n}$ is the nominal area of fusion face normal to the tensile force $\left(\mathrm{mm}^{2}\right)$. 
The weld was assumed to be all around the perimeter of the steel angle. For most of the studied cases, the size of the weld was $5 \mathrm{~mm}$ (3/16 inch). For some cases the size of the weld was increased to $20 \mathrm{~mm}(3 / 4 \mathrm{inch})$ in order to resist the reactions at the connection.

\subsection{Suggested VSS sections (Single-layer steel plate)}

The results shows that some VSS shields with specific container sizes can contain specific charges. The following table summarize the results listed before and it shows what sections can be used to contain a given TNT charge. Table 14 shows a summary for the results obtained. The term "failure" in the table indicates that a failure occurs to the steel member and that the section wasn't able to withstand the load. Also the table shows the weld size that was used to connect the steel plates with the main frame of the container.

Table 14: Summary for the deflections of mid-span for steel plates for different cases

\begin{tabular}{|c|c|c|c|c|c|c|}
\hline $\begin{array}{c}\text { TNT } \\
\text { Charge, } \\
(\mathrm{kg})\end{array}$ & $\begin{array}{l}\text { Size of the } \\
\text { container, }(\mathrm{m})\end{array}$ & $\begin{array}{l}\text { Vent } \\
\text { area } \\
\text { ratio }\end{array}$ & $\begin{array}{c}\text { Length of } \\
\text { steel plate, } \\
\quad(\mathrm{mm})\end{array}$ & $\begin{array}{l}\text { Min Steel } \\
\text { Plate } \\
\text { Thickness, } \\
(\mathrm{mm})\end{array}$ & $\begin{array}{c}\text { Max } \\
\text { deflection, } \\
(\mathrm{mm})\end{array}$ & $\begin{array}{l}\text { Weld type - } \\
\text { Size, }(\mathrm{mm})\end{array}$ \\
\hline 1 & $2.0 \times 2.0$ & 0.25 & 150 & 7 & 199.97 & $E 43 X X-5$ \\
\hline 1 & $2.0 \times 2.0$ & 0.50 & 100 & 7 & 191.00 & $E 43 X X-5$ \\
\hline 1 & $3.0 \times 3.0$ & 0.25 & 150 & 10 & 197.08 & $E 43 X X-5$ \\
\hline 1 & $3.0 \times 3.0$ & 0.50 & 100 & 10 & 195.93 & $E 43 X X-5$ \\
\hline 1 & $4.0 \times 4.0$ & 0.25 & 200 & 6 & 354.03 & $E 43 X X-5$ \\
\hline 1 & $4.0 \times 4.0$ & 0.50 & 200 & 5 & 405.00 & $E 43 X X-5$ \\
\hline 5 & $2.0 \times 2.0$ & 0.25 & 150 & 21 & 203.79 & Failure \\
\hline 5 & $2.0 \times 2.0$ & 0.50 & 100 & 19 & 211.26 & Failure \\
\hline 5 & $3.0 \times 3.0$ & 0.25 & 150 & 17 & 201.85 & $E 43 X X-5$ \\
\hline 5 & $3.0 \times 3.0$ & 0.50 & 100 & 16 & 205.44 & $E 43 X X-5$ \\
\hline 5 & $4.0 \times 4.0$ & 0.25 & 200 & 23 & 204.65 & $E 43 X X-5$ \\
\hline
\end{tabular}




\begin{tabular}{|c|c|c|c|c|c|c|}
\hline $\begin{array}{c}\text { TNT } \\
\text { Charge, } \\
(\mathrm{kg})\end{array}$ & $\begin{array}{c}\text { Size of the } \\
\text { container, (m) }\end{array}$ & $\begin{array}{l}\text { Vent } \\
\text { area } \\
\text { ratio }\end{array}$ & $\begin{array}{l}\text { Length of } \\
\text { steel plate, } \\
\quad(\mathrm{mm})\end{array}$ & $\begin{array}{l}\text { Min Steel } \\
\text { Plate } \\
\text { Thickness, } \\
(\mathrm{mm})\end{array}$ & $\begin{array}{c}\text { Max } \\
\text { deflection, } \\
(\mathrm{mm})\end{array}$ & $\begin{array}{l}\text { Weld type - } \\
\text { Size, (mm) }\end{array}$ \\
\hline 5 & $4.0 \times 4.0$ & 0.50 & 200 & 22 & 206.48 & $E 43 X X-5$ \\
\hline 10 & $2.0 \times 2.0$ & 0.25 & 150 & 30 & 206.13 & Failure \\
\hline 10 & $2.0 \times 2.0$ & 0.50 & 100 & 28 & 207.78 & Failure \\
\hline 10 & $3.0 \times 3.0$ & 0.25 & 150 & 15 & 210.49 & $E 43 X X-5$ \\
\hline 10 & $3.0 \times 3.0$ & 0.50 & 100 & 14 & 298.56 & $E 43 X X-5$ \\
\hline 10 & $4.0 \times 4.0$ & 0.25 & 200 & 24 & 204.43 & $E 43 X X-5$ \\
\hline 10 & $4.0 \times 4.0$ & 0.50 & 200 & 23 & 207.93 & $E 43 X X-5$ \\
\hline 20 & $2.0 \times 2.0$ & 0.25 & 150 & 71 & 121.50 & Failure \\
\hline 20 & $2.0 \times 2.0$ & 0.50 & 100 & 60 & 144.51 & Failure \\
\hline 20 & $3.0 \times 3.0$ & 0.25 & 150 & 55 & 153.65 & Failure \\
\hline 20 & $3.0 \times 3.0$ & 0.50 & 100 & 52 & 160.48 & Failure \\
\hline 20 & $4.0 \times 4.0$ & 0.25 & 200 & 28 & 407.66 & $E 43 X X-5$ \\
\hline 20 & $4.0 \times 4.0$ & 0.50 & 200 & 28 & 281.77 & $E 43 X X-5$ \\
\hline
\end{tabular}

It can be seen from the results that a charge of $20 \mathrm{~kg}$ TNT cannot be contained in any of the suggested sections. The section of the plate can be increased $71 \mathrm{~mm}$ which will result in having a very heavy section, however, when the steel plate is exceed to that limit, the failure occurs at the welding part because the it can't resist the end support reactions.

\subsection{Suggested VSS sections (Single-layer side-by-side angle interior-facing)}

As previously mentioned, using steel angles is more efficient that using steel plates in terms of attenuation and load resistance. The Single-layer side-by-side angle interior-facing was found to be better than the exterior facing configuration in terms of blast attenuation, so this 
section will be studied in this section to find out the appropriate sections that can be used to resist specific charges inside the container. The following table (Table 15) shows the sections that are proposed to be used to attenuate internal blast loads. The vent area ratio was kept constant equals to 0.5 for reducing the number of variables. The two parameters are the charge weight and the size of the container. The table also include the size of the weld that need to be used to support the steel angles.

Table 15: Summary for the deflections of mid-span for Single-layer side-by-side angle interior-facing for different load cases

\begin{tabular}{|c|c|c|c|c|}
\hline $\begin{array}{l}\text { TNT Charge, } \\
(\mathrm{kg})\end{array}$ & $\begin{array}{l}\text { Size of the container, } \\
(\mathrm{m})\end{array}$ & $\begin{array}{l}\text { Vent area } \\
\text { ratio }\end{array}$ & Steel angle & $\begin{array}{c}\text { Weld type - Size, } \\
(\mathrm{mm})\end{array}$ \\
\hline 1 & $2.0 \times 2.0$ & 0.50 & L19x19x3.2 & $E 43 X X-5$ \\
\hline 1 & $3.0 \times 3.0$ & 0.50 & L19x19x3.2 & $\mathrm{E} 43 \mathrm{XX}-5$ \\
\hline 1 & $4.0 \times 4.0$ & 0.50 & L19x19x3.2 & $\mathrm{E} 43 \mathrm{XX}-5$ \\
\hline 5 & $2.0 \times 2.0$ & 0.50 & $\mathrm{~L} 44 \times 44 \times 6.4$ & $\mathrm{E} 43 \mathrm{XX}-5$ \\
\hline 5 & $3.0 \times 3.0$ & 0.50 & L $44 \times 44 \times 6.4$ & $\mathrm{E} 43 \mathrm{XX}-5$ \\
\hline 5 & $4.0 \times 4.0$ & 0.50 & L44×44x6.4 & $E 43 X X-5$ \\
\hline 10 & $2.0 \times 2.0$ & 0.50 & L51x51x9.5 & $E 43 X X-5$ \\
\hline 10 & $3.0 \times 3.0$ & 0.50 & L51×51x9.5 & $\mathrm{E} 43 \mathrm{XX}-5$ \\
\hline 10 & $4.0 \times 4.0$ & 0.50 & L51×51x9.5 & $\mathrm{E} 43 \mathrm{XX}-5$ \\
\hline 20 & $2.0 \times 2.0$ & 0.50 & L102x102x19 & $E 43 X X-5$ \\
\hline 20 & $3.0 \times 3.0$ & 0.50 & L51x51x9.5 & $\mathrm{E} 43 \mathrm{XX}-5$ \\
\hline 20 & $4.0 \times 4.0$ & 0.50 & L76x76x9.5 & $E 43 X X-5$ \\
\hline
\end{tabular}

\subsection{Comparison between configurations}

From the obtained results, it can be concluded that the single and multilayer VSS containers could be used to attenuate blast pressure outside those containers. The attenuation of 
blast load of VSS containers depends mainly on the (effective) vent area ratio, so the required level of attenuation could be obtained by changing the vent area ratio. In this section, the performance of the studied VSS sections with the same vent area ratio is compared. The comparison is in terms of level of attenuation, weight, elimination of fragments and structural resistance. The weights of the four parameters are set to be equal due to the importance of the four of them. Each configuration gets a grade out of 1 for each parameter and a total grade of 4 is calculated for each geometric section.

From the results obtained in this study, it was found that the level of attenuation of VSS sections depends on the effective vent area ratio as well as the geometric configuration. In general, the double layer VSS sections showed a better attenuation than the single layer configurations. Therefore, the two double layer configurations got a grade of 1 . Following the two double layer configurations, the Single layer side-by-side angle (interior facing) shows a better performance than the other single layer configurations followed by the single layer plate configuration, so they get a grade of 0.8 and 0.7 , respectively. The Single layer side-by-side angle (exterior facing) comes in the last place with a grade 0.6 .

Elimination of fragments is very important objective of using VSS containers. All single layer VSS section allows direct paths for fragments to escape outside the VSS container which is considered a disadvantage of the single layer configurations. Therefore, all the single layer sections are given a grade of 0.5 out of 1 for this disadvantage. However, this disadvantage is not applicable in case of double layer VSS sections which completely prevent fragments from escaping outside. As a result, double layer VSS sections get a grade of 1 in this comparison.

The weight of the double layer VSS sections are almost the same weight of the single layer with the same vent area ratio. However, an additional $20 \%$ is added to the weight of the container in case of the double layer configurations to take into the account the effect of the overlap between the steel rows as they are arranged in a staggered arrangement as shown in Figure 112. Consequently, the grade of Double Layer Single plate configuration and the DoubleLayer Nested Side-By-Side Angle were reduced by 0.2 in the comparison than the single layer plate and the Single layer side-by-side angle, respectively. The single layer plate configuration is the lightest between the studied sections so it gets a grade of 1 . The two sections; Single layer 
side-by-side angle (exterior facing) and (exterior facing) are heavier than the single plate configuration so they get a grade of 0.9 .

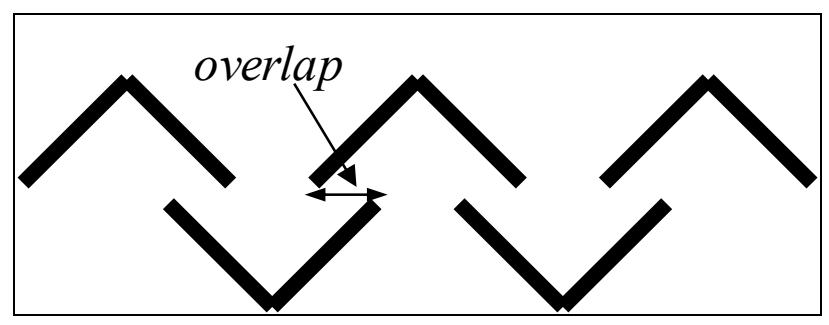

Figure 112: The effect of overlap on the weight

Structural resistance of steel angles in the Single layer side-by-side angle (exterior facing) and Single layer side-by-side angle (interior facing) configurations was found to be stronger than the steel plates used in the Single layer steel plate. Therefore, the steel plate configurations get a lower grade than those of the steel angle configurations. Table 16 shows a comparison between the different studied configurations.

Table 16: Comparison between VSS configurations

\begin{tabular}{|c|c|c|c|c|c|c|}
\hline Configuration & Geometric configuration & Attenuation & $\begin{array}{c}\text { Direct } \\
\text { Paths }\end{array}$ & Weight & $\begin{array}{c}\text { Structural } \\
\text { resistance }\end{array}$ & Total \\
\hline $\begin{array}{c}\text { Single layer } \\
\text { single plate }\end{array}$ & 0.7 & 0.5 & 1 & 0.4 & 2.6 \\
\hline $\begin{array}{c}\text { Single layer } \\
\text { side-by-side } \\
\text { angle } \\
\text { (exterior } \\
\text { facing) }\end{array}$ \\
\hline $\begin{array}{c}\text { Single layer } \\
\text { side-by-side } \\
\text { angle } \\
\text { (interior } \\
\text { facing) }\end{array}$
\end{tabular}


From the table, it can be concluded that the Double-Layer Nested Side-By-Side Angle configuration gets the highest grade between the studied configurations. So as a conclusion, this section is considered to be the most feasible configuration that could be used for VSS sections.

\subsection{Summary}

This chapter addressed the structural design of steel members of the VSS side walls. A SDOF model was developed to calculate the structural response the steel members due to internal explosion. The applied load was the pressure profile obtained earlier in chapter 4 . The response of the steel members were recorded and compared to the response obtained from AUTODYN 3D models. The SDOF showed a good prediction for the steel member response due to internal blast loading.

The SDOF was also capable to calculate the reactions at the end supports of the steel members. These reactions were then used in the design of the welding part that connects the steel members to the main frame of the container. Finally, some VSS containers were introduced to attenuate a specific amount of charge. The introduced sections included the geometric configuration, dimensions and welding sizes.

Finally a comparison was carried out between the studied VSS sections. This comparison included all the parameters that were studied in the thesis. The comparison concluded that the Double-Layer Nested Side-By-Side Angle showed the best configuration that can be used in VSS containers. This conclusion coincides with the same conclusion that was obtained by Elshafey (2008) where it was stated that this section is the optimum VSS section that could be used. 


\section{Chapter 7: Conclusions and Recommendation for Future Work}

\subsection{Introduction}

In this thesis, numerical and analytical investigations were carried out to determine the effect of internal explosion inside steel vented suppressive shield (VSS) containers on the structural members of the container. The thesis included both 2D and 3D modeling using AUTODYN commercial software. The $2 \mathrm{D}$ modeling was studied to investigate the propagation of the blast waves through different sections of suppressive shields as well as the interaction between blast waves and structural elements. From the 2D models, the pressure values were recorded outside the container as well as the pressure on the steel angles forming the side walls of the container. Subsequently, the 3D modelling part was the focus of the next stage, where different sections of the suppressive shield were modelled to investigate the structural response of the steel angles due to the applied load.

Finally, a Single degree of freedom (SDOF) analytical model was introduced and developed to predict the structural response of the side walls of the VSS container when subjected to internal explosion. The model was able to predict the structural response of the steel angles. The conclusions obtained from the study as well as the recommended future work are presented in the following sections.

\subsection{Conclusions}

The main conclusions obtained from the research can be summarized as follows:

- A reliable design methodology was developed to analyze and design the elements of Vented Suppressive Shield (VSS) containers. The developed method can be used to predict the structural response of the VSS elements without the use of Computational Fluid Dynamics (CFD) software packages.

- The developed design methodology includes a set of equations that were developed to predict the pressure and Impulse outside VSS shields. The pressure values obtained from the developed equations were in good agreement with those measured from previous experimental work. 
- The design methodology can be used to calculate the blast load profile on the side wall of vented structures.

- The equation proposed by Cox et al. (1978) was examined and found to be valid to calculate the effective vent area ratio for multilayer VSS systems, if the ratio of the gap between the layers to the gap between the layers of the VSS configuration equals to or less than 0.5 . If this condition is not satisfied, then the equation will not give an accurate calculation for the effective vent area ratio and thus the performance of the section cannot be predicted.

- Pressure profile on the elements of the VSS containers depends on the geometric shape of the VSS section. This effect was quantified by proposing geometric coefficients for different VSS sections, in order to predict the pressure profile on irregular VSS configurations.

- The developed SDOF model is able to predict the deflections of the VSS steel elements in comparison to the values obtained from AUTODYN output. The SDOF model takes into consideration the effect of tension membrane action that occurs in steel elements due to large deformations.

\subsection{Future Research Work}

This research developed a design methodology to analyze and design VSS elements of VSS containers. However further research work is required in order to optimise the design approach for these containers and verify the results experimentally. The following sections discuss several suggestions for future work that needs to be carried out.

\subsection{Effect of Fragments}

In most cases of stored dangerous goods, they are contained in solid cases. In case of accidental explosion, the solid parts fracture and fly with a very high speed known as fragments. The effect of fragments on the VSS container elements was not addressed in this thesis, however studying the effect of fragmentations is important as it may affect the design of the VSS sections in order to resist the effect of fragmentations. 


\subsection{Composite materials}

As mentioned before; the main objective of this research is to design a VSS section that is light in weight and can be used in the transportation process. In order to reduce the weight of the section, lighter materials could be used. Composite materials could be an efficient substitution for the steel material. The composite materials are less in density and at the same time possess a material resistance close to that of the steel.

\subsection{Analytical model}

The developed model was able to predict the behaviour of the steel angles and plates that are subjected to blast loads. The model assumed that the steel angles are connected with the main frame of the container by a fixed connection. However, in reality, the main frame is also exposed to a part of the load from the internal explosion that causes deformation in the main frame and thus it cannot be considered as a FULLY fixed support. More investigations need to be done to take into account the effect of the deformation of the main frame on the structural behaviour of the steel angles.

\subsection{Welding connection}

In some cases that were studied in the thesis, the weld part was not able to carry the load at the connections between the VSS elements and the main frame of the container. This is due to the high reactions that were experienced at the end supports. Further investigation need to be carried out in order to study the possibility of having a better connection between the steel angles and the main frame. 


\section{References}

Above top secrets, "Village Blown up in Fireworks Explosion in Denmark", Visited in May, 2013, Retrieved from: http://www.abovetopsecret.com/forum/thread94829/pg2

ASCE, "Blast Protection of Buildings (ASCE/SEI 59-11)", American Society of Civil Engineers, 2011.

Baker W. E., Westine P. S., “Methods Of predicting Blast Loads Inside And Blast Fields Outside Suppressive Structures" Department of the Army, November 1975.

Baker, W. E., Cox, P. A., Kulesz, J. J., Strehlow, R. A., \& Westine, P. S., “Explosion hazards and evaluation", 1983.

BBC News, "Dutch Fireworks Disaster", BBC, 14 May 2000, Visited in November, 2013. Retrieved from: http://news.bbc.co.uk/2/hi/europe/747566.stm.

BBC news, "Lorry blast kills 16 in Romania", Visited in August, 2012, Retrieved from: http://news.bbc.co.uk/2/hi/europe/3742161.stm

Birnbaum Naury K., Tancreto James, Hager Kevin, “Calculation of Blast Loading in the High Performance Magazine with AUTODYN-3D”, Naval Facilities Engineering Service Center, Port Hueneme, CA, August 1994.

Birnbaum, Naury K., Francis, Nigel J., \& Gerber, Bence I., "Coupled Techniques for the Simulation of Fluid-Structure and Impact Problems", Computer Assisted Mechanics and Engineering Sciences, 1999, Vol. 6, n. 3-4, pp. 295-311.

Blodgett, Omer W., "Design of welded structures", The James F. Lincoln Arc Welding foundation, 2002.

Børvik T., Hanssen A.G., Dey S., Langberg H., Langseth M., "On the ballistic and blast load response of a $20 \mathrm{ft}$ ISO container protected with aluminium panels filled with a local massPhase I: Design of protective system”, Engineering Structures 30 (2008), pp. 1605-1620. 
Børvik T., Hanssen A.G., Dey S., Langberg H., Langseth M., "On the ballistic and blast load response of a $20 \mathrm{ft}$ ISO container protected with aluminium panels filled with a local massPhase II: Validation of protective system”, Engineering Structures 30 (2008) 1621-1631.

Braimah Abass, Elshafey Mohamed, Abd El Halim O. Abd El Halim, and Contestabile Ettore, "Experimental Investigation of Aluminum Foam Lined Suppressive Shield Containment Vessels", Protective Structures, Vol. 3, Number 2, June 2012.

Braimah Abass, "Course CIVE 5707 notes, Effect of explosives on Structures", Lecture 2, Carleton University, 2012

Carbiener Kevin E., "Preliminary Results for a Russian Designed Explosive Resistant Container", Sandia National Laboratories, Livermore, CA, November 1996.

Carre G. L, Walker R. E., “Airblast Loading of a Large Metal Shipping Container”, U. S. Army Engineer Waterways Experiment Station, Vicksburg, Mississippi, July 1970

Century Dynamics Inc., "Interaction tutorial”, Revision 4.3, 2005.

Century Dynamics Inc., "Remapping tutorial”, Revision 4.3, 2005.

Century Dynamics Inc., "ANSYS AUTODYN in Workbench", 2005

CISC, "Handbook of steel Construction", Canadian Institute of Steel Construction, Eighth edition, December, 2004.

CNN Wire Staff. "19 Killed in Fireworks Explosion in Northeast China", Cable News Network (CNN), 16 August 2010, Visited in November, 2013, Retrieved from:http://www.cnn.com/2010/WORLD/asiapcf/08/16/china.fireworks.explosion/index.html

Cox P. A., Esparza E. D., "Preliminary Design of a Suppressive Structure for a Melt-Loading Operation", Southwest Research Institute, May 1976.

Cox P. A., Westine P. S., Kulesz J. J., Esparza E. D., “Analysis and Evaluation of Suppressive Shields", Southwest Research Institute, January 1978 
CSB, "Explosives Manufacturing Incident", Investigation report no. 98-001-I-NV, U.S. Chemical Safety and Hazard Investigation Board, 1998.

Demotix, "Explosion at the Evangelos Florakis Naval Base in Cyprus - Zygi", Visited in January, 2013, Retrieved from: http:/www.demotix.com/photo/752688/explosion-evangelosflorakis-naval-base-cyprus-zygi

Department of the Army, U.S. Army Corps of Engineering, "Suppressive Shields Structural Design and Analysis Handbook”, Huntsville Division, November 18, 1977.

Eco container, "Exploded axonometric view of a typical 20' ISO Shipping Container", Visited in December, 2012, Retrieved from: http://www.ecocontainerhome.com/resources/explodedaxonometric-view-of-a-typical-20-iso-shipping-container/

Esparza E. D., Baker W. E., Oldham G. A., "Blast pressures Inside and Outside Suppressive Structures", EM-CR-76042, Report No. 8, Department of the Army, December 1975.

El-Magd, E. "Mechanical properties at high strain rates", Le Journal de Physique IV 4. C8 (1994): C8-149.

Elshafey Mohamed, "Experimental and Numerical investigations for the development of safe transportation of hazardous materials", Ph.D. thesis, Department of Civil and Enviromental Engineering Department, Carleton University, 2008.

Elshafey M. M., Contestabile E., Abd El Halim A. O., and Isgor O. B., “An Experimental Study Improving the Safety of Transportation of Dangerous Goods", Transportation Research Record 2009.

Royal Wolf, "Explosive Magazines", Visited in November 2013, Retrieved from:http://www.royalwolf.com.au/specialty-shipping-containers/explosive-magazines/

Fairlie G. E., "The Numerical Simulation of High Explosives using AUTODYN - 2D \& 3D", Explo '98, Institute of Explosive Engineers 4 ${ }^{\text {th }}$ Biannual Symposium, September 1998.

Fišerová Darina, "Numerical Analyses of Buried Mine Explosions with Emphasis on Effect of Soil Properties on Loading", PHD Thesis, Cranfield University, January 2006. 
Gould Kenneth E., Tempo Kaman, "High-Explosive Field tests", Explosion Phenomena and Environmental Impacts", contract no: DNA 001-79-C-0053, October 1981.

Hancock, J. W. and Mackenzie, A.C, "On the mechanism of ductile failure in high strength steels subjected to multi-axial stress-states", Journal of Mechanism and Physics of Solids, 1976, pages 147-169.

IMO, "International Maritime Dangerous Goods Code (IMDG CODE)", International Maritime Organization, 1998.

Katsanis, David J., "Vented Suppressive Shielding (VSS) In Explosive Operations", Shielding Technologies, Inc., pp. 1681-1692.

Kasemets, Juri T., "Quantity-Distance and Explosives", Explosives Regulatory Division Explosives Safety and Security Branch Minerals and Metals Sector, Natural Resources of Canada, March 2008.

Kingery, C. N. and Bulmash, G., "Airblast Parameters from TNT Spherical Air Bursts and Hemispherical Surface Bursts", ARBRL-TR-02555, April 1984.

King, P.V., Becher, A.F., and Henderson, W.P., "Blast Suppressive Shielding”, United States Patent number 4248342, February 3rd, 1981.

Kinney, G. F. and Graham, K. J., 1985, “Explosive Shocks in Air”, Springer-Verlag, New York Inc., NY, USA.

Kivity Y, Florie C, Lenselink H, "Response of protective structures to internal explosion with blast venting", - MSC World Users Conference, Arlington, VA, 1993.

Kloster Mark S., "Coupled Lagrangian and Eulerian Approach to Detonation and Fragmentation Problems", Research Office Naval Postgraduate School Monterey, September 2001.

Koger, D. M. and McKown, G. L., "Category 5 Suppressive Shield Test Report", Edgewood Arsenal Technical Report EM-TR-76001, Oct 1975. 
Lee et al, "JWL Equation of State Coefficients for High Explosives", Lawrence Livermore Lab, UCID-16189, January 1973.

Lee Y. W. and Wierzbicki T., "Fracture prediction of thin plates under localized impulsive loading. Part I: dishing", International Journal of Impact Engineering 2005, 31(10): 1253-1276.

Majzoobi, G.H., Ghom, A., "Optimization of autofrettage in thick walled cylinders", Journal Achievements in Materials and Manufacturing Engineering, Volume 16, 2006.

Maienschein J. L., "Estimating Equivalency of Explosives through a Thermo-chemical Approach", 12th International Conference Symposium, San Diego, California, July 8, 2002.

Maruyama Xavier, K., "Explosive Detection Technologies for Airline Security", Institute for Joint Warfare Analysis and Dept. of Physics Naval Postgraduate School, Monterey, California, 2000.

Naval Facilities Engineering Service Center, "AUTODYN-3D”, Naval Facilities Engineering Service Center, Port Hueneme, CA, AUG 1994.

Needham, Charles E. “Blast Wave Propagation”, Springer Berlin Heidelberg, 2010.

Lam, Nelson, Priyan Mendis, and Tuan Ngo, "Response spectrum solutions for blast loading" Electronic Journal of Structural Engineering 4 (2004): 28-44.

New York Times news, "Fireworks Truck Explodes shattering a Bridge in China", Visited in May, 2013, Retrieved from: http://www.nytimes.com/2013/02/02/world/asia/fireworks-truckexplodes-on-bridge-in-china.html?_r=0

Olson, M. D., G. N. Nurick, and J. R. Fagnan, "Deformation and rupture of blast loaded square plates - predictions and experiments", International Journal of Impact Engineering 13.2 (1993): 279-291.

Paik Jeom Kee, Thayamballi Anil K., Lee Sang Kon, Kang Sung Jun, “A semi-analytical method for the elastic-plastic large deflection analysis of welded steel or aluminum plating under combined in-plane and lateral pressure loads", Thin-Walled Structures 39 (2001) 125-152. 
Palley Igor, Gary Allan Harpell, and Max Wilhelm Gerlach, "Blast resistant and blast directing containers and methods of making" U.S. Patent No. 6,991,124, 31 Jan. 2006.

Paz, Mario, "Structural dynamics: theory and computation”, Springer, 1997.

Provencher M., "Road transport of explosives small or large quantities", Transport Canada, Transport Dangerous Goods Directorate, Ottawa, Ontario, Canada, May 2005

Unified Facilities Criterial (UFC 3-340-02), "Structures to Resist the Effects of Accidental Explosions", US Army Corps of Engineers, Naval Facilities Engineering Command, and Air Force Civil Engineer Support Agency, 2008.

US Chemical storage, "Convertible ISO shipping containers", Visited in May, 2013, Retrieved from: http:/www.uschemicalstorage.com/convertible-iso-shipping-containers/

US Department of Defense, "DOD Ammunition and Explosives Safety Standards: General Quantity-Distance Criteria for Accidental Detonations", NUMBER 6055.09-M, Volume 3, Department of Defense, United States of America, February 29, 2008.

Rajendran, A. M., Grove, D.J., Dietenberger, M. A., Cook, W. H., “A Dynamic Failure Model for Ductile Materials", Air Force Armament Laboratory, February 1991.

Richard J. Mainiero and James H. Rowland III, "A Review of Recent Accidents Involving Explosives Transport", NIOSH, Pittsburgh Research Laboratory, Pittsburgh, PA, 2009.

Royal Golf news, “Australia's largest Shipping \& Storage Container Provider", Visited in May, 2013, Retrieved from: http:/www.royalwolf.com.au/defence/explosive-magazines/

Seraji Mahmood, Wan Badaruzzaman W.H., Osman S. A., "Membrane action in profiled steel sheeting dry board (PSSDB) floor slab system", Journal of Engineering Science and Technology, Vol. 8, No. 1 (2013) $57-68$.

Shipping containers website, "ISO Shipping Containers", Visited in May 2013, Retrieved from: http://www.shippingcontainers24.com/general/iso-standardization/\#axzzlplRZFDxI

Sochet I., "Blast effects of external explosions", "Eighth International Symposium on Hazards, Prevention, and Mitigation of Industrial Explosions, Yokohama, Japan, 2010. 
Swisdak Jr, Michael M., "Simplified Kingery airblast calculations", Naval Surface Warfare Center Indian Head Div MD, 1994.

Ventsel, Eduard, and Theodor Krauthammer, "Thin plates and shells: theory: analysis, and applications", CRC press, 2001.

Wharton R.K, Formby S.A, Merrifield R, “Air blast TNT equivalence for a range of commercial blasting explosives", Journal of Hazardous Materials, Vol. 79, December 2000, Pages 31-39.

Zukas, Jonas, "Introduction to hydrocodes", Access Online via Elsevier, 2004. 


\section{Appendix A: Previous Accidents}

\section{1) Fireworks factory explosion in northeast China}

On August $16^{\text {th }}, 2010$, an explosion occurred in a fireworks factory in northeast China. The factory was not in operation when the explosion occurred so the exact number of workers in the factory at the time of the explosion was unknown. The explosion was so strong that the shockwave was felt up to five kilometers away (CNN World, 2010). The explosion caused the death of 33 people and the injury of 153. A preliminary investigation showed that the factory was illegally producing fireworks, as its production permit was confiscated in June 2010 by the local work safety department. Figure 113 shows the smoke and fire remaining after the explosion (CNN World, 2010).

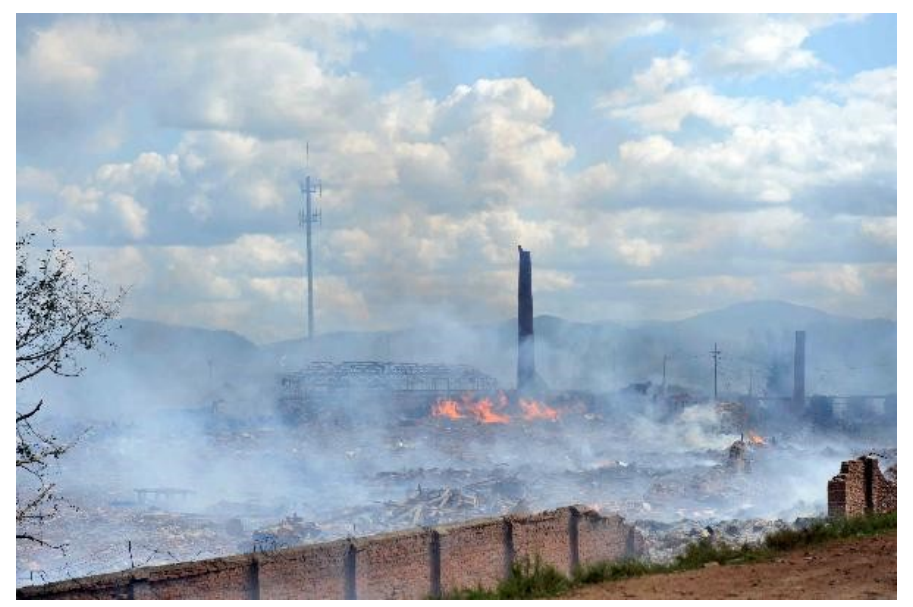

Figure 113: Smoke and fire remaining after the explosion (CNN news, 2010)

\section{2) Enschede fireworks explosion}

The Enschede fireworks explosion occurred at the fireworks depot on May 13, 2000, in the eastern part of the Netherland. The fire began in the work area of the central building where about $900 \mathrm{~kg}$ of fireworks was stored. The fire then extended to two containers that had been illegally stored outside the building. The fire eventually led to their ignition causing the explosion of 177 tons of fireworks. The explosion resulted in the destruction of buildings and infrastructure within a 40 hectare area around the warehouse. About 400 houses were incinerated 
and over 1,500 houses suffered minor damage. 1,250 residents were rendered homeless and thousands more had to be evacuated. The total loss resulting from this accident including houses and lives was believed to be more than 450 million US dollars (BBC news, 2000).

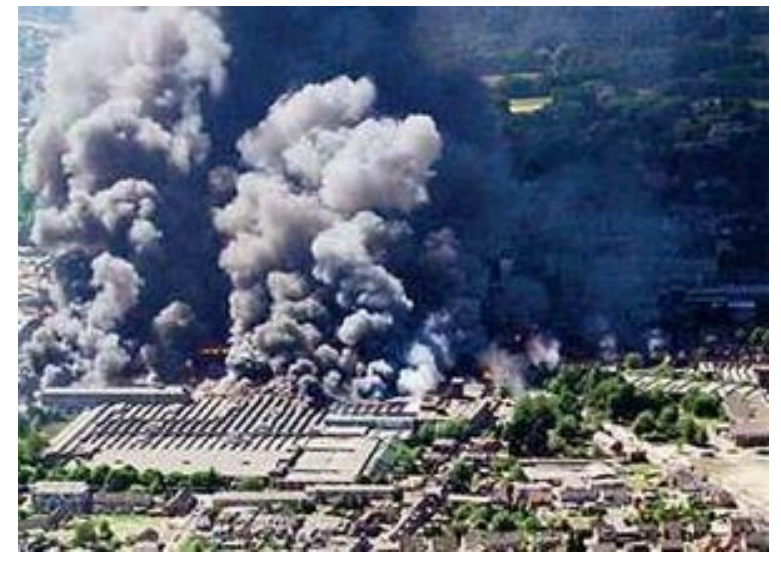

Figure 114: Smoke from the fire, (BBC news, 2000)

\section{3) Kean Canyon explosives manufacturing plant explosion}

Kean Canyon explosives manufacturing plant is owned by Sierra Chemical Company. Two massive explosions occurred a few seconds apart in the manufacturing plant on January $7^{\text {th }}$, 1998, killing four workers and injuring six others. The initial explosion occurred in a room of one of the buildings where workers made explosive "boosters". Another building that was located 70m away contained Pentaerythritol Tetranitrate (PETN) which is a highly unstable high explosive which is often transported wet to mitigate the potential of unplanned explosion. The initial explosion resulted in the initiation of the PETN explosives inside and resulting in a secondary explosion. This explosion caused a crater of 12 meters in diameter with a depth of 2 metres. In this accident, the explosives in the second building are considered as secondary explosive; however they had more effect than the primary explosives (the boosters). Figure 115 shows the fire after the massive explosion (U.S. Chemical safety and hazard investigation board, 1998). 


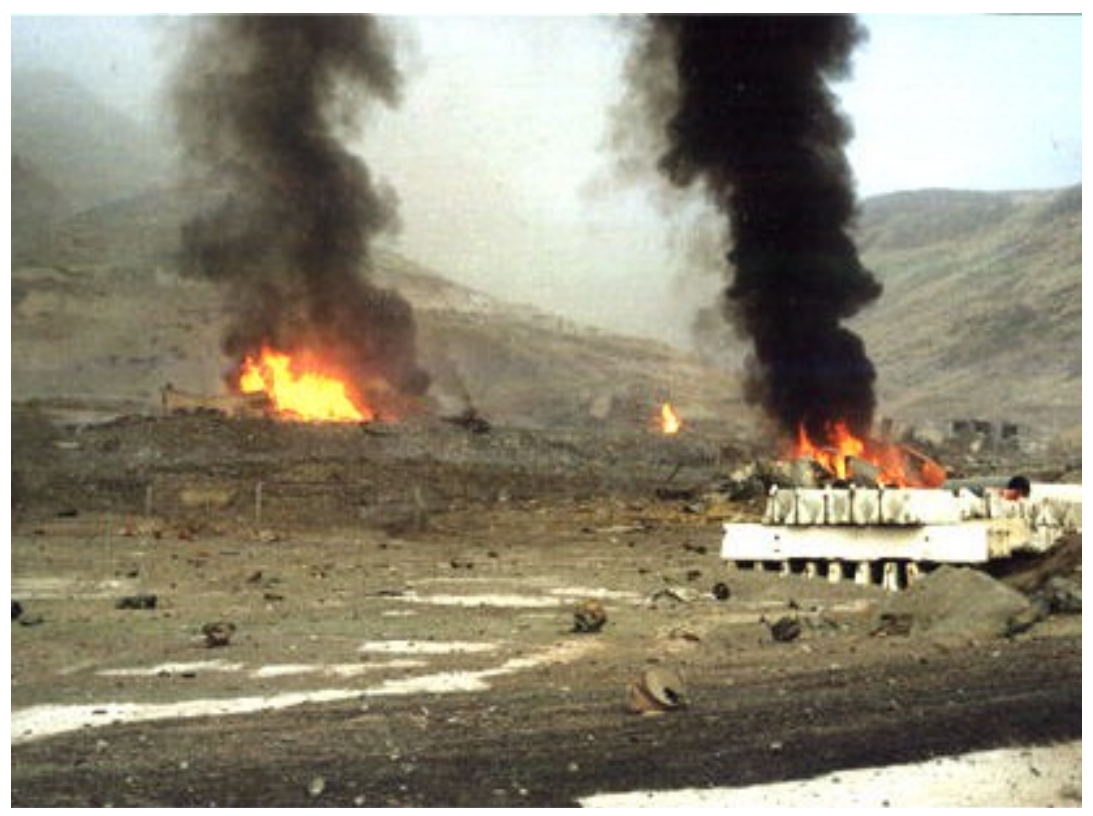

Figure 115: Sierra Chemical facility burns after massive explosions (U.S. Chemical safety and hazard investigation board, 1998)

\section{4) Seest fireworks explosion}

The fireworks accident in Seest happened on November 03, 2004, when a fireworks factory exploded in Seest, a suburb of Kolding, Denmark. The post-event investigation concluded that the factory stored more fireworks than was allowed by regulation. The cause of the accident was attributed to two workers who accidentally dropped a box containing fireworks while working inside a container. The impact caused the fireworks to ignite. When the fire crew arrived they initially thought that they were dealing with a simple container fire. However, the fire was too intense, and they were unsuccessful in extinguishing it before the explosion took place. In this accident, one firefighter died and seven members of the rescue team as well as 17 locals were injured. The evacuation of 2,000 people from the immediate surroundings of the factory saved many lives. Eight fire and rescue vehicles were also destroyed. 


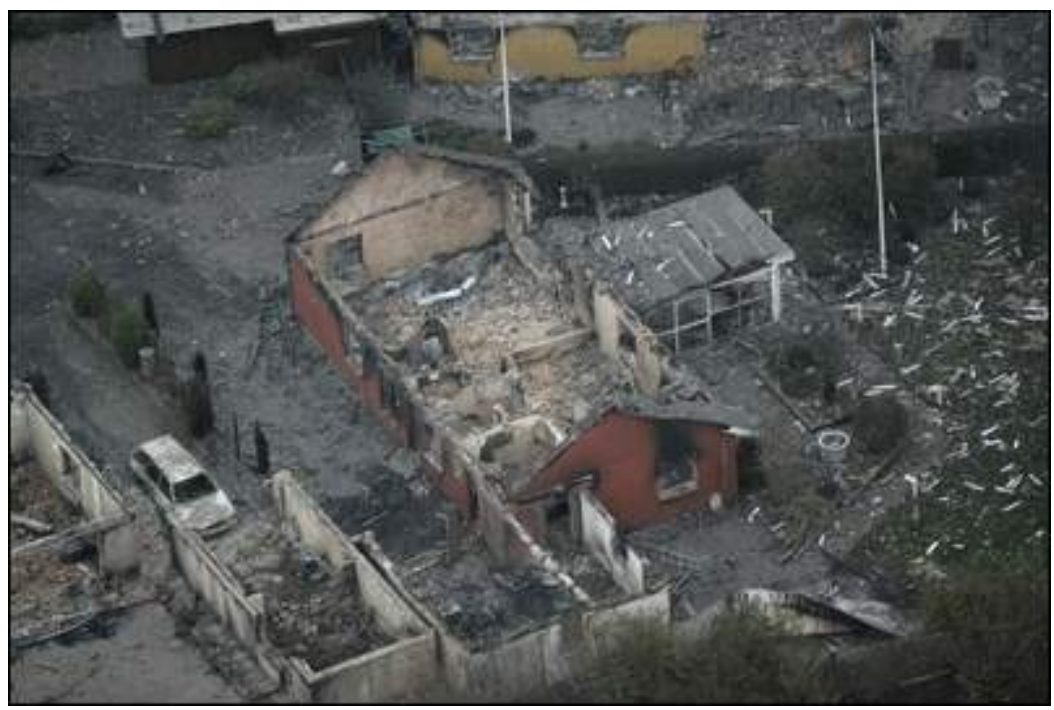

Figure 116: Seest fireworks explosion (Above top secrets, 2006)

\section{5) Truck Explosion in Romania}

On May 24, 2004 a truck carrying more than 22 tons of "nitrous fertilizers" was involved in a single vehicle accident $50 \mathrm{~km}$ northeast of Bucharest, Romania. The driver tried to extinguish the fire; failing, he asked for help at a nearby village. A few cars stopped and some curious on-lookers watched the fire together with a television news crew. Firefighters arrived at the accident site and while preparing to fight the fire, the truck exploded (BBC news, 2004). The truck had burnt for about an hour after the original truck accident. The truck explosion killed 20 people, including 7 firefighters, 2 journalists, and 8 on-lookers. This accident not only caused loss of lives but also affected the traffic flow for about 2 weeks for reconstruction. The truck explosion left a $6.5 \mathrm{~m}$ deep and $42 \mathrm{~m}$ wide crater. The loss of life and damage to the road could have been reduced if the accident site was evacuated after the nitrous fertilizer started to burn. This accident is a prime example of regulations failure (BBC news, 2004). 


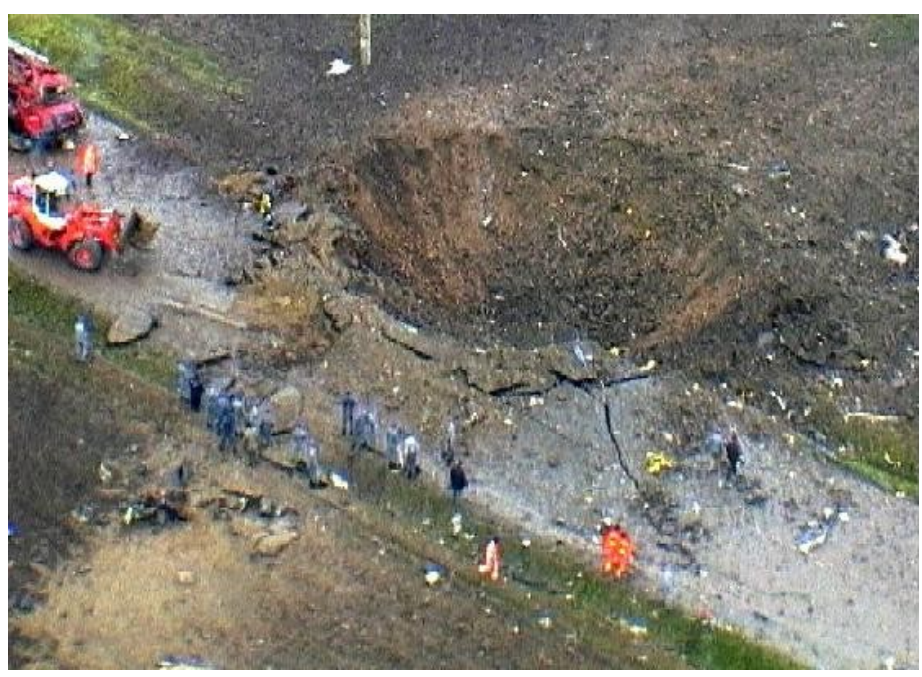

Figure 117: Crater resulted from explosion (BBC news, 2004)

\section{6) Truck Explosion in Walden, Ontario}

On August 5, 1998 an explosives truck carrying 18,000 $\mathrm{kg}$ of blasting explosives went off the road and struck a rock face near Walden, Ontario, Canada. The accident started a fire and other drivers passing by stopped to help the driver exit the truck. The truck exploded about 35 minutes after the accident. The explosion caused two minor injuries, threw fragments of the truck up to $2.74 \mathrm{~km}$, and damaged several houses. At the time the truck exploded firefighters were in contact with the Canadian Transport Emergency Centre (CANUTEC) and were advised against approaching the scene. This accident is a good example of how to deal with accidents involving explosives, in contrast to the accident in Romania. The evacuation of the accident site prevented fatalities or serious injuries. Figure 118 shows the damage that occurred due to the explosion while Figure 119 shows a shift in the asphalt due to the power of the explosion. 


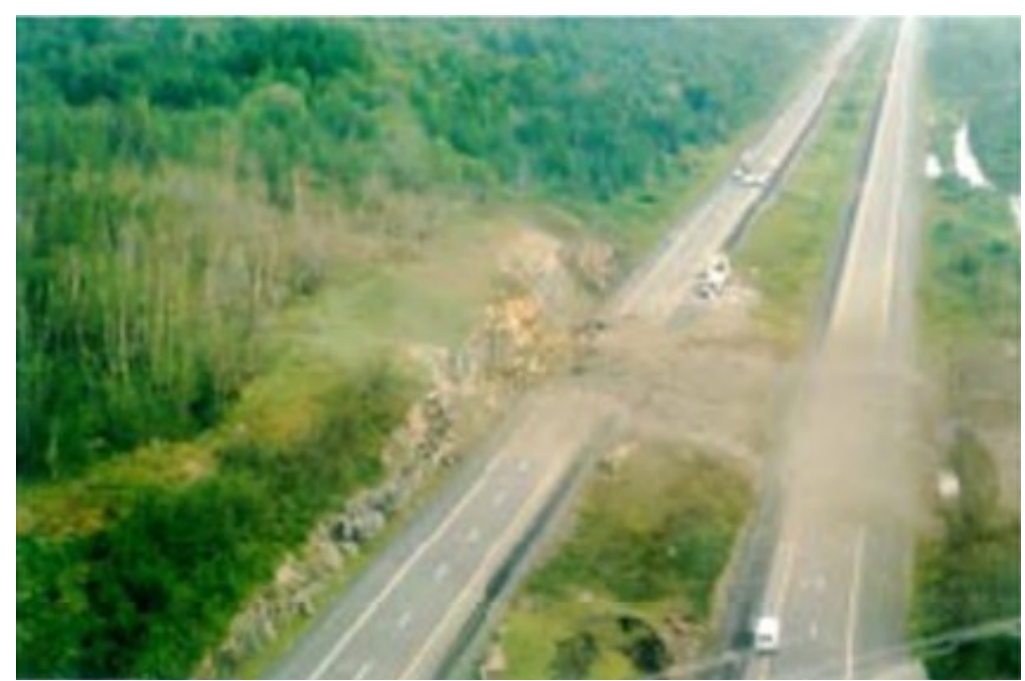

Figure 118: Aerial photograph of the accident site (Mainiero et al, 2009)

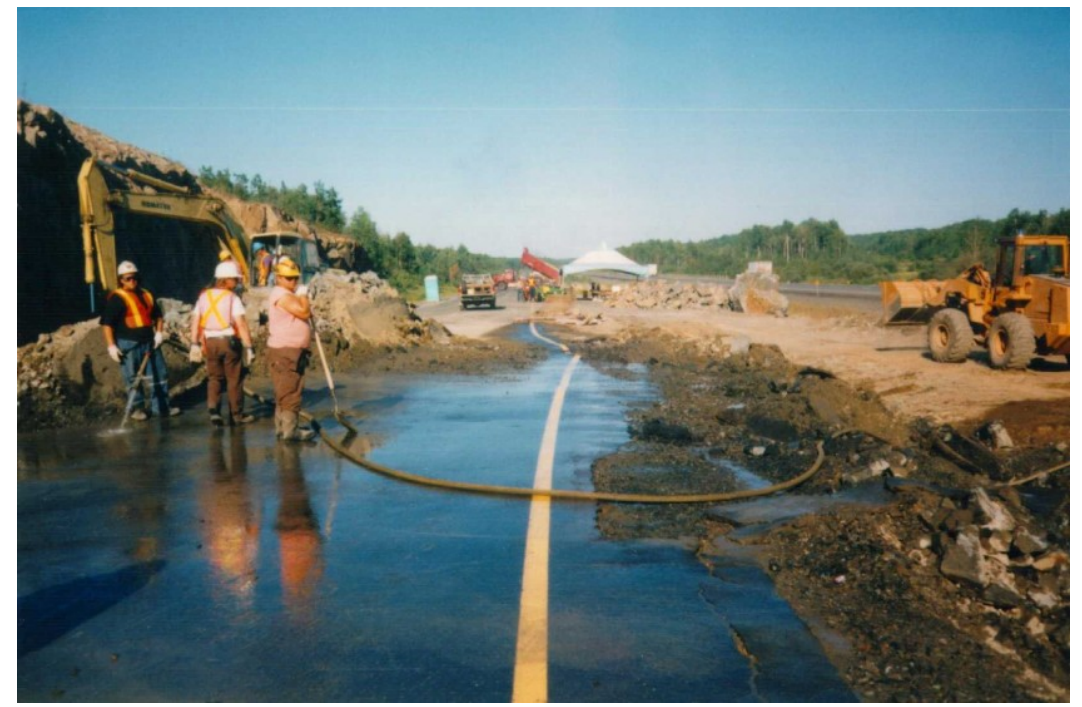

Figure 119: Highway asphalt shifts as a result of the huge explosion (Provencher, 2005) 


\section{Appendix B: Computational methods}

$$
\begin{aligned}
& F U N C T I O N=E X P\left(A+B^{*} L N(Z)+C^{*}(L N(Z))^{\wedge} 2+D^{*}(L N(Z))^{\wedge} 3+E^{*}(L N(Z))^{\wedge} 4\right. \\
& \left.+F^{*}(L N(Z))^{\wedge} 5+G^{*}(L N(Z))^{\wedge} 6\right)
\end{aligned}
$$

\begin{tabular}{|c|c|c|c|c|c|c|c|c|}
\hline & $Z$ value & A & B & $\mathrm{C}$ & $\mathrm{D}$ & $\mathrm{E}$ & $\mathrm{F}$ & $\mathrm{G}$ \\
\hline \multirow{2}{*}{$\begin{array}{c}\text { Arrival time } \\
\left(\mathrm{msec} / \mathrm{Kg}^{\wedge}(1 / 3)\right)\end{array}$} & $0.06-1.5$ & -0.7604 & 1.8058 & 0.1257 & -0.0437 & -0.031 & $\begin{array}{c}- \\
0.00669\end{array}$ & 0 \\
\hline & $1.5-40$ & -0.7137 & 1.5732 & 0.5561 & -0.4213 & 0.1054 & $\begin{array}{c}- \\
0.00929 \\
\end{array}$ & 0 \\
\hline \multirow{3}{*}{$\begin{array}{c}\text { Incident } \\
\text { pressure }(\mathrm{kPa})\end{array}$} & $0.2-2.9$ & 7.2106 & $\begin{array}{c}- \\
2.1069\end{array}$ & -0.3229 & 0.1117 & 0.0685 & 0 & 0 \\
\hline & $2.9-23.8$ & 7.5938 & $\begin{array}{c}- \\
3.0523 \\
\end{array}$ & 0.40977 & 0.0261 & $\begin{array}{c}- \\
0.01267 \\
\end{array}$ & 0 & 0 \\
\hline & $\begin{array}{l}23.8- \\
198.5 \\
\end{array}$ & 6.0536 & $\begin{array}{c}- \\
1.4066\end{array}$ & 0 & 0 & 0 & 0 & 0 \\
\hline \multirow{2}{*}{$\begin{array}{c}\text { Reflected presure } \\
(\mathrm{kPa})\end{array}$} & $0.06-2$ & 9.006 & 2.6893 & -0.6295 & 0.1011 & 0.29255 & 0.13505 & $\begin{array}{c}0.01973 \\
6 \\
\end{array}$ \\
\hline & $2.0-40$ & 8.8396 & -1.733 & -2.64 & 2.293 & -0.8232 & 0.14247 & -0.0099 \\
\hline \multirow{3}{*}{$\begin{array}{c}\text { Positive phase } \\
\text { duration } \\
\left(\operatorname{msec} / \operatorname{Kg}^{\wedge}(1 / 3)\right)\end{array}$} & $0.2-1.02$ & 0.5426 & 3.2299 & -1.5931 & -5.9667 & -4.0815 & -0.9149 & 0 \\
\hline & $\begin{array}{l}1.02- \\
2.80 \\
\end{array}$ & 0.544 & 2.7082 & -9.7354 & 14.3425 & -9.7791 & 2.8535 & 0 \\
\hline & $2.8-40$ & -2.4608 & 7.1639 & -5.6215 & 2.2711 & $\begin{array}{c}- \\
0.44994 \\
\end{array}$ & 0.03486 & 0 \\
\hline \multirow{4}{*}{$\begin{array}{c}\text { Incident Impulse } \\
\left(\mathrm{kPa} \cdot \mathrm{msec} / \mathrm{Kg}^{\wedge}(1 / 3)\right. \\
)\end{array}$} & $0.2-0.96$ & 5.522 & 1.117 & 0.6 & -0.292 & -0.087 & 0 & 0 \\
\hline & $\begin{array}{l}0.96- \\
2.38\end{array}$ & 5.465 & -0.308 & -1.464 & 1.362 & -0.432 & 0 & 0 \\
\hline & $\begin{array}{l}2.38- \\
33.7 \\
\end{array}$ & 5.2749 & $\begin{array}{c}- \\
0.4677 \\
\end{array}$ & -0.2499 & 0.0588 & $\begin{array}{c}- \\
0.00554 \\
\end{array}$ & 0 & 0 \\
\hline & $\begin{array}{l}33.7- \\
158.7 \\
\end{array}$ & 5.9825 & -1.062 & 0 & 0 & 0 & 0 & 0 \\
\hline $\begin{array}{c}\text { Reflected Impulse } \\
\left(\mathrm{kPa} \cdot \mathrm{msec} / \mathrm{Kg}^{\wedge}(1 / 3)\right. \\
)\end{array}$ & $0.06-40$ & 6.7853 & 1.3466 & 0.101 & 0.01123 & 0 & 0 & 0 \\
\hline \multirow{2}{*}{$\begin{array}{l}\text { Shock front velocity } \\
(\mathrm{km} / \mathrm{sec})\end{array}$} & $0.06-1.5$ & 0.1794 & -0.956 & -0.0866 & 0.0109 & 0.0699 & 0.01218 & 0 \\
\hline & $1.5-40$ & 0.2597 & -1.326 & 0.3767 & 0.0396 & -0.0351 & 0.00432 & 0 \\
\hline
\end{tabular}

Where A, B, C, D, E, F, G are the coefficients given below:

After obtaining the previous parameters, the pressure profile is drawn using the equation below:

$$
P(t)=\text { Coefficient } * P_{s o}\left(1-\frac{t}{t_{d}}\right) e^{\frac{-b}{t_{d}}}
$$




\section{Appendix C: Results for the prediction of pressure profiles}

This appendix illustrates the verification of the developed equations to calculate the pressure on the steel angles of VSS sections. The verification was done by comparing the pressure profile obtained from the introduced equations and those obtained from AUTODYN. The following cases are for single layer steel plate VSS containers. The differences between the showed cases are the TNT charge inside the container, the size of the container and the vent area ratio of the section. The pressure was calculated steel plates

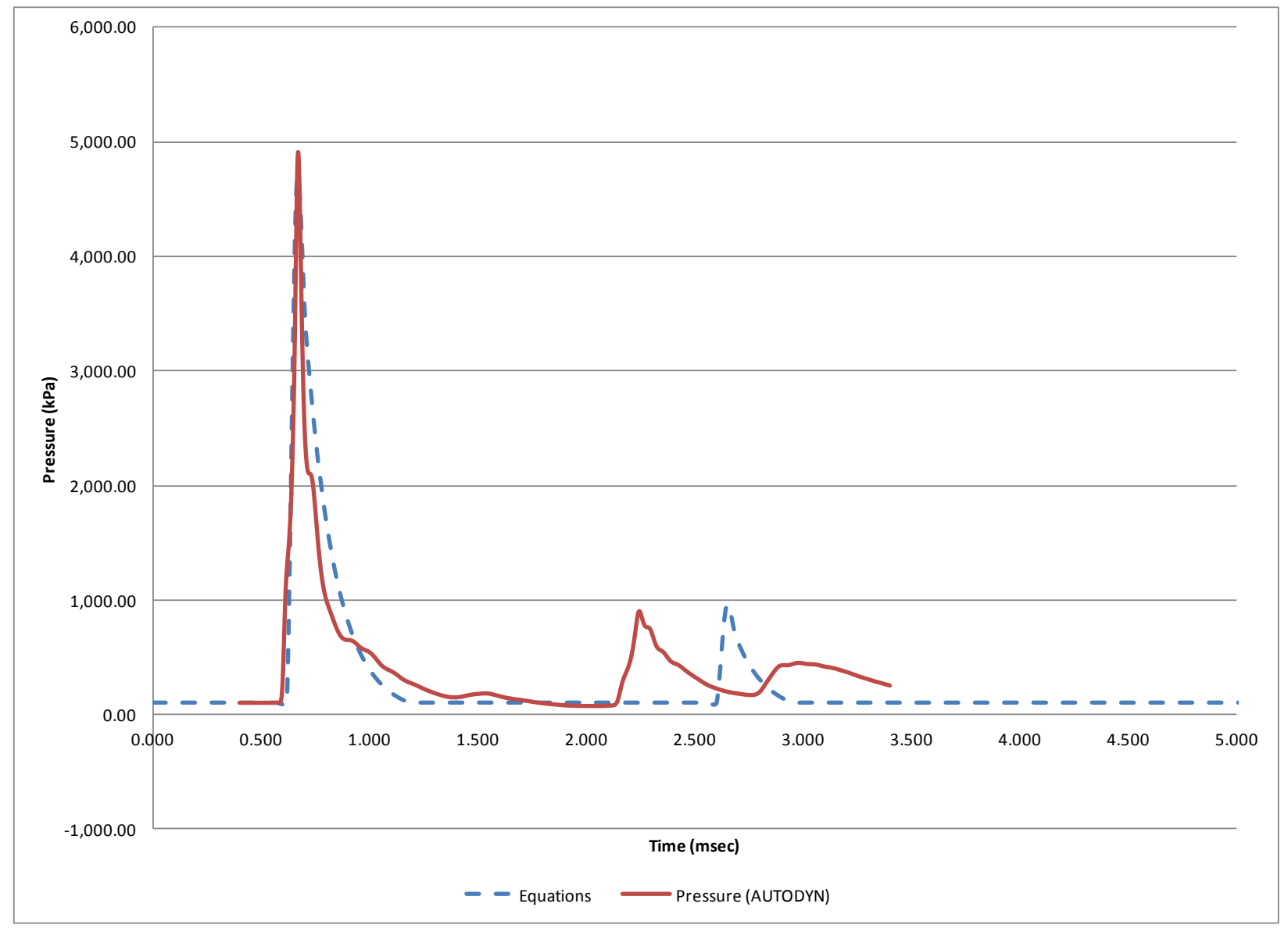

Figure 120: Pressure profile for $1 \mathrm{~kg}$ TNT charge in a $(2.0 \times 2.0)$ container with 0.25 vent area ratio 


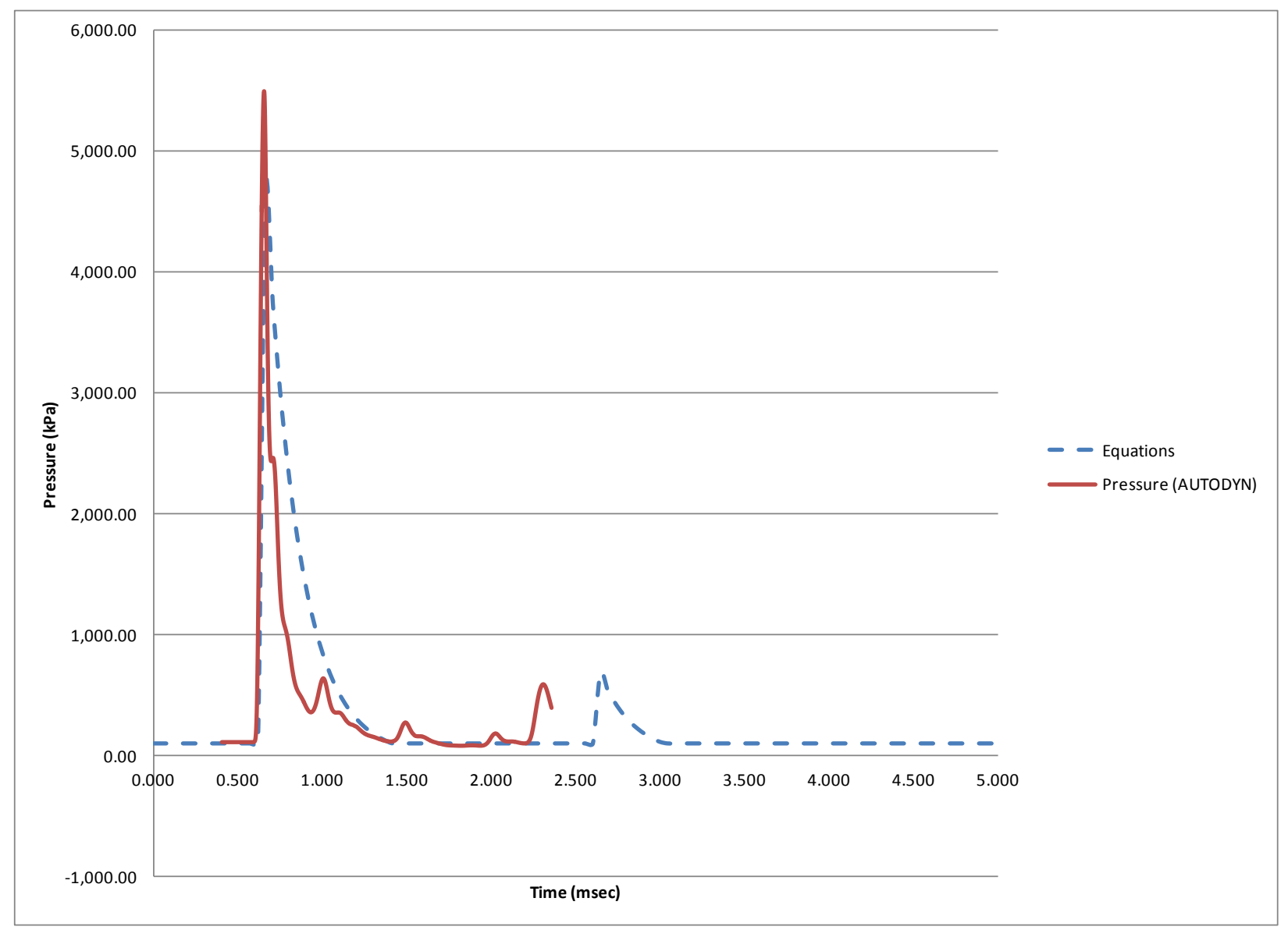

Figure 121: Pressure profile for $1 \mathrm{~kg}$ TNT charge in a $(2.0 \times 2.0)$ container with 0.5 vent area ratio 


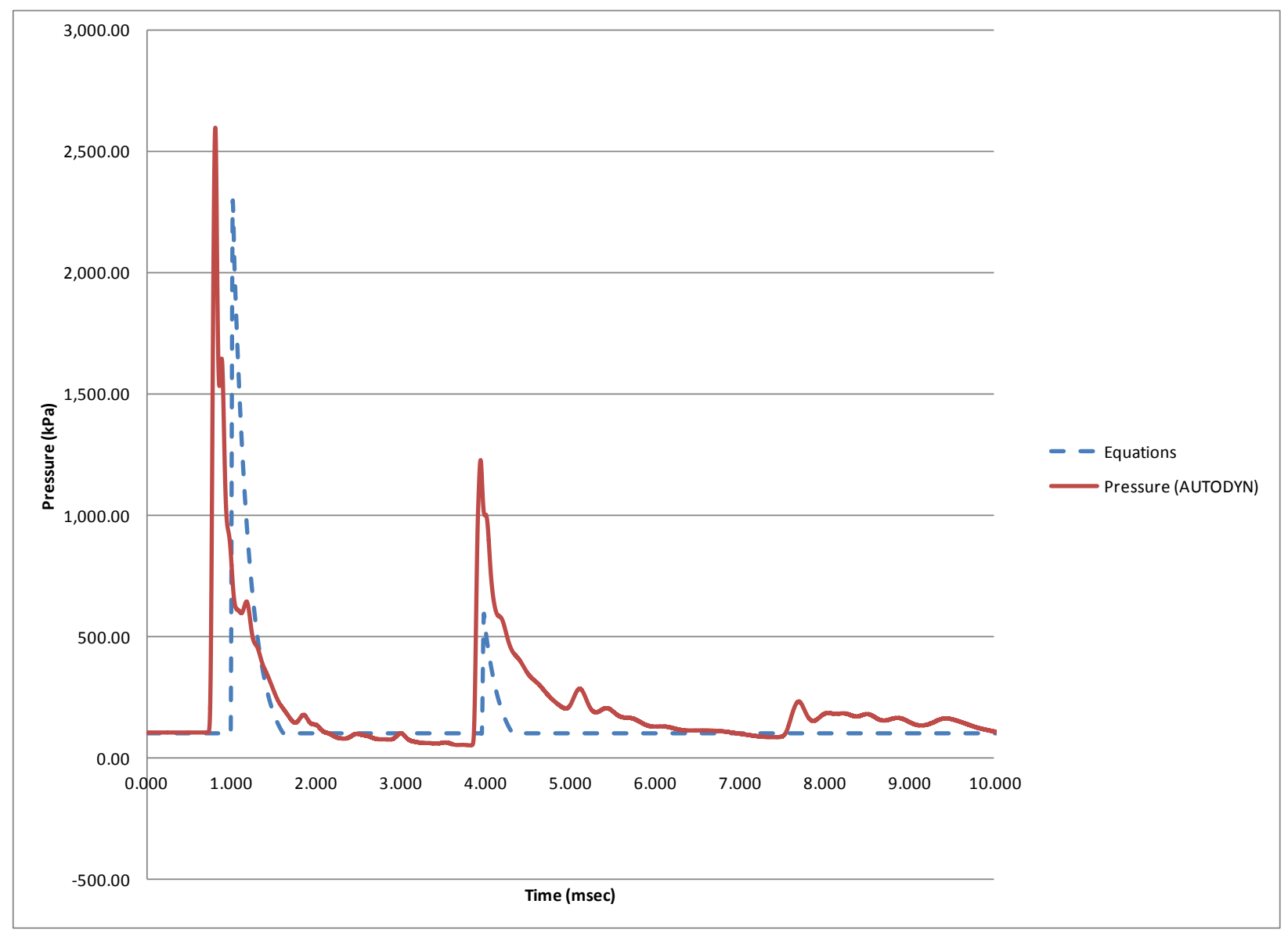

Figure 122: Pressure profile for $1 \mathrm{~kg}$ TNT charge in a $(3.0 \times 3.0)$ container with 0.25 vent area ratio 


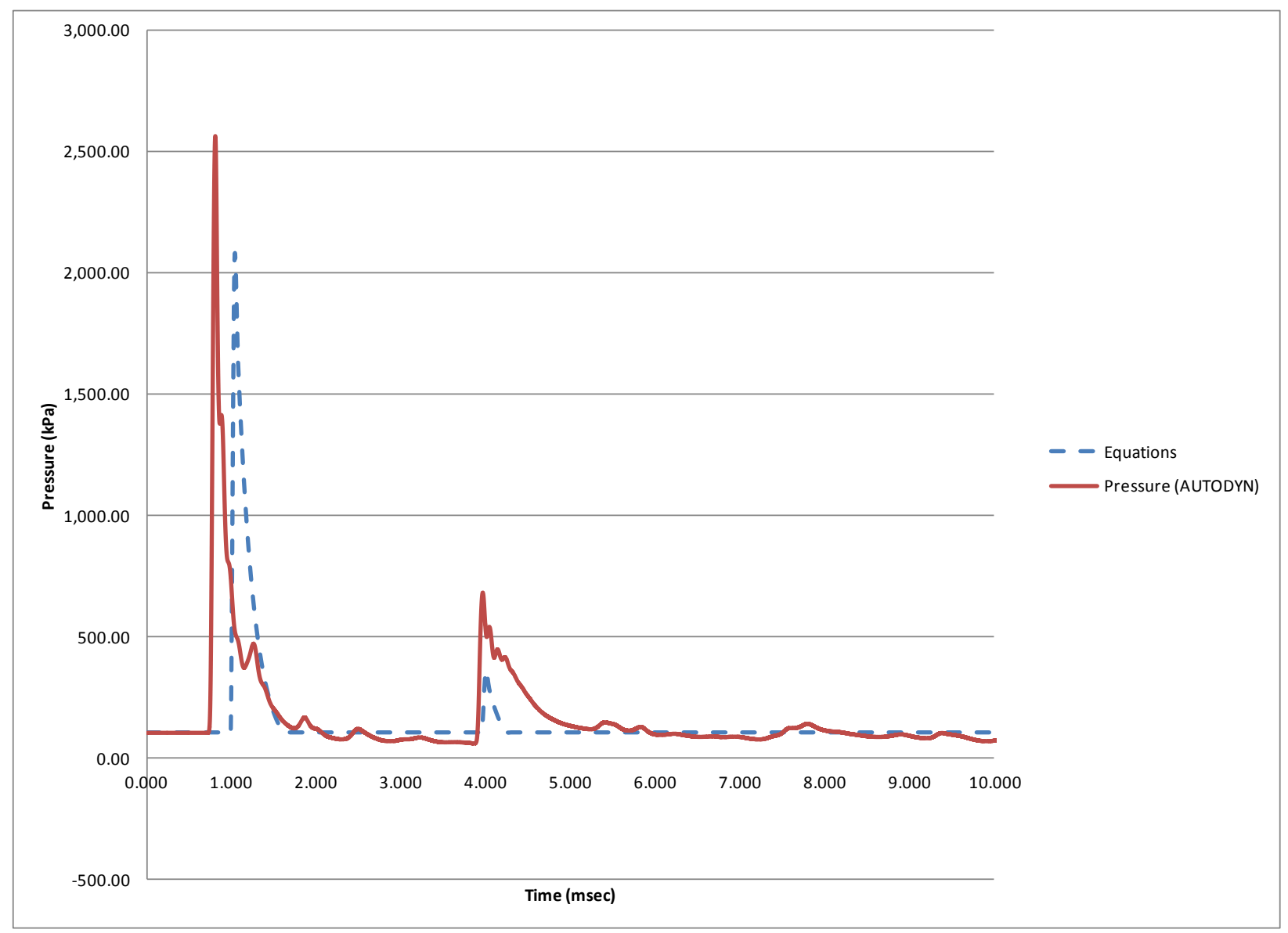

Figure 123: Pressure profile for $1 \mathrm{~kg}$ TNT charge in a $(3.0 \times 3.0)$ container with 0.5 vent area ratio 


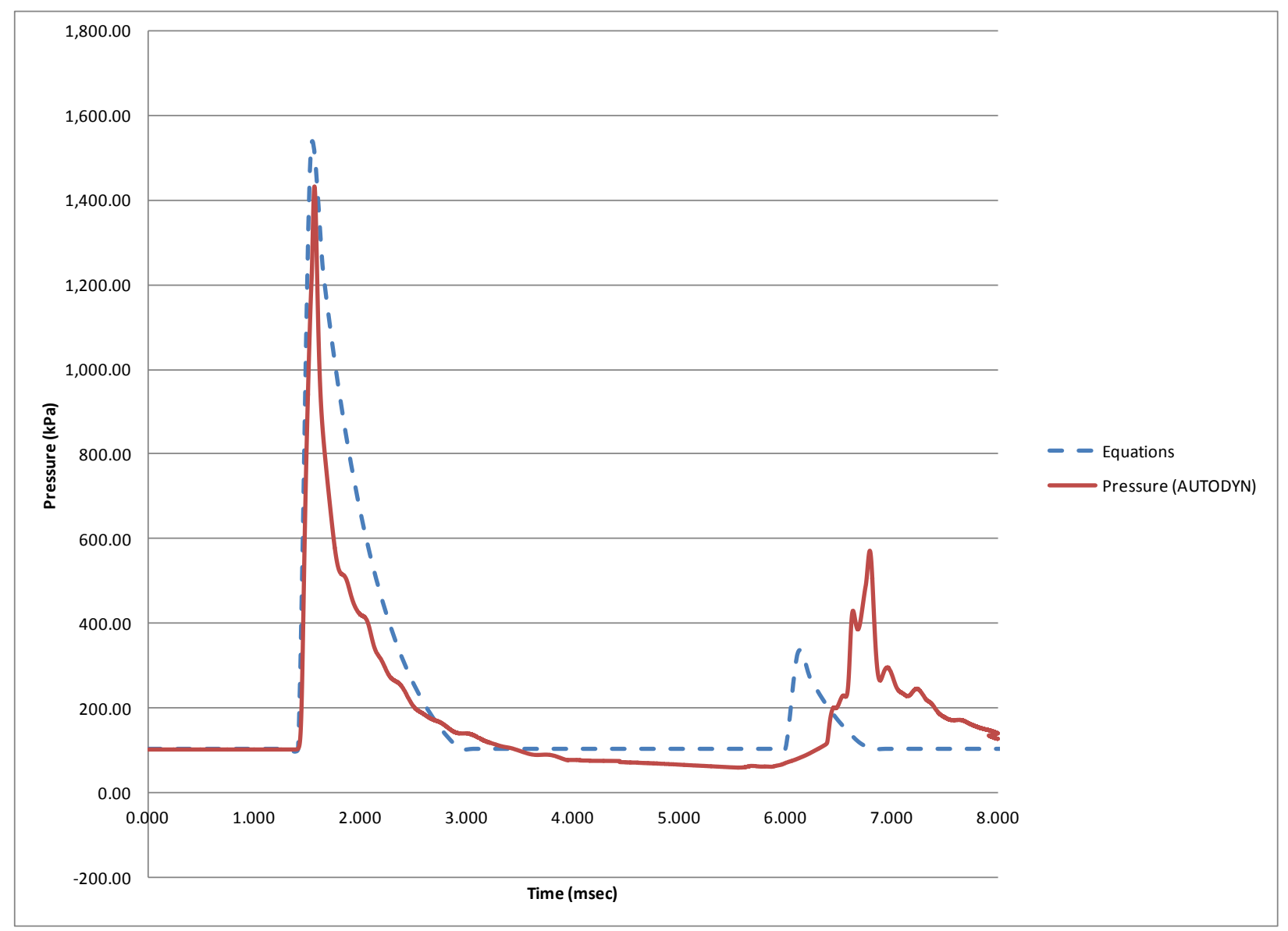

Figure 124: Pressure profile for $1 \mathrm{~kg}$ TNT charge in a $(4.0 \times 4.0)$ container with 0.25 vent area ratio 


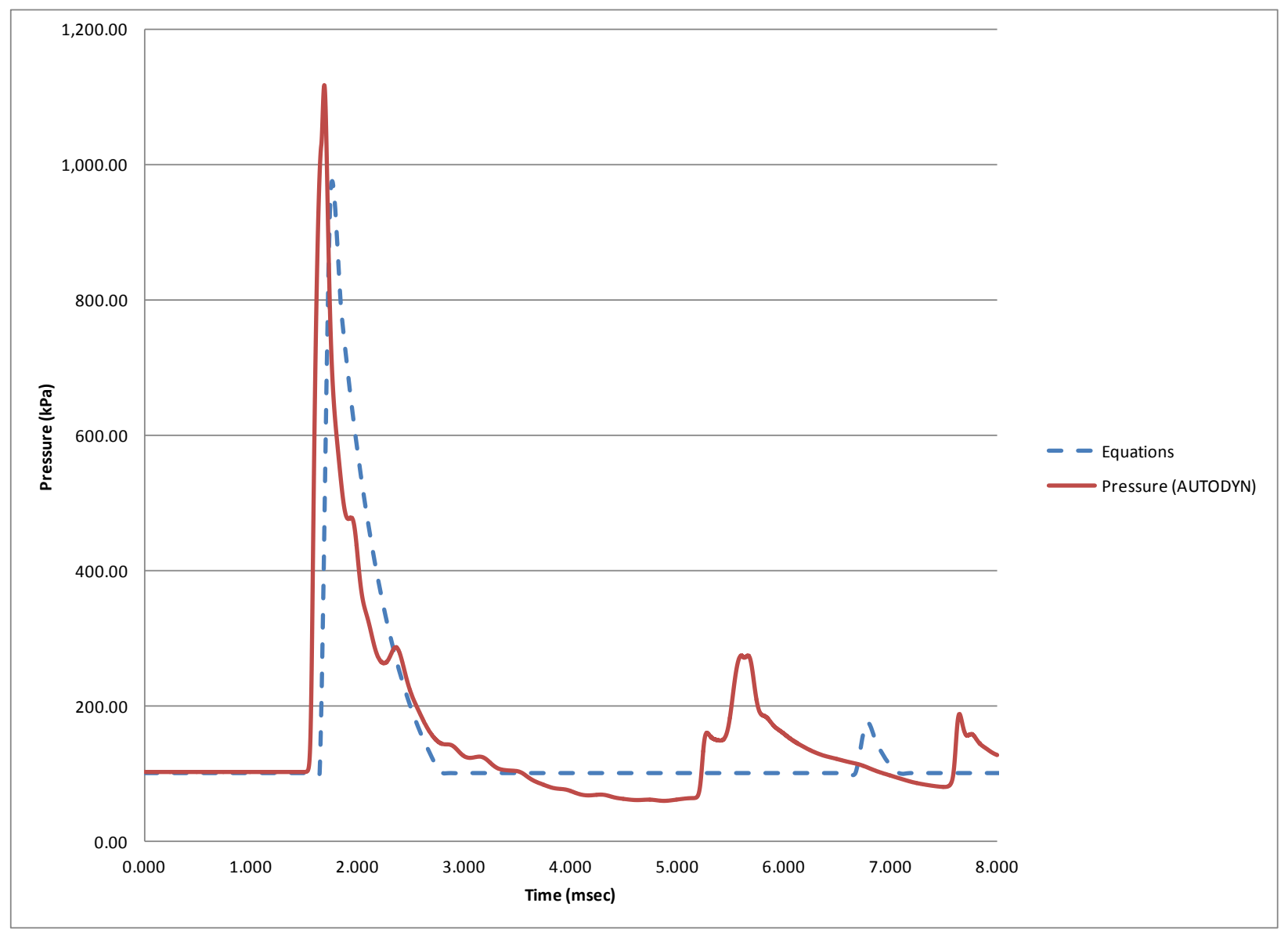

Figure 125: Pressure profile for $1 \mathrm{~kg}$ TNT charge in a $(4.0 \times 4.0)$ container with 0.5 vent area ratio 


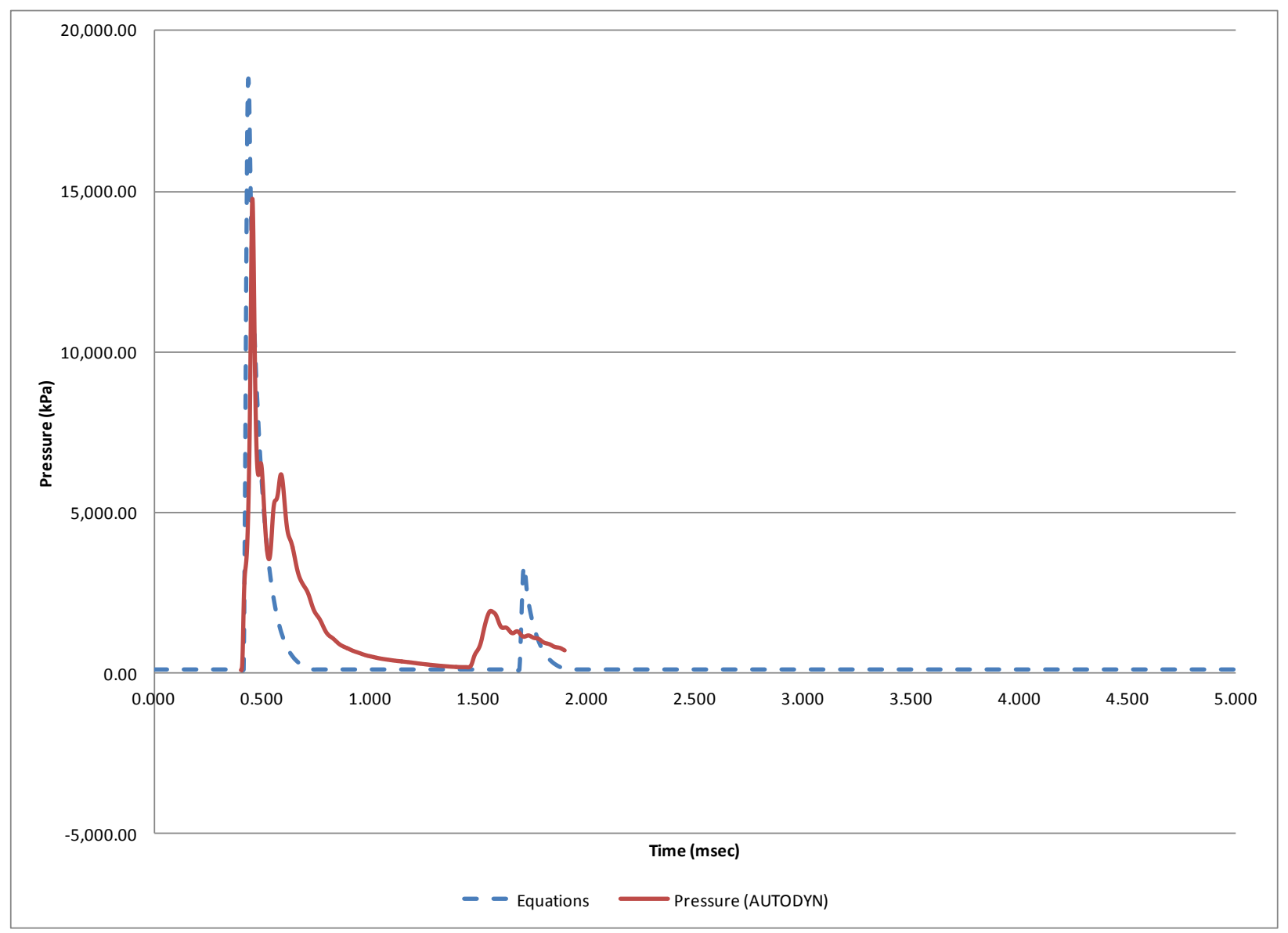

Figure 126: Pressure profile for $5 \mathrm{~kg}$ TNT charge in a $(2.0 \times 2.0)$ container with 0.25 vent area ratio 


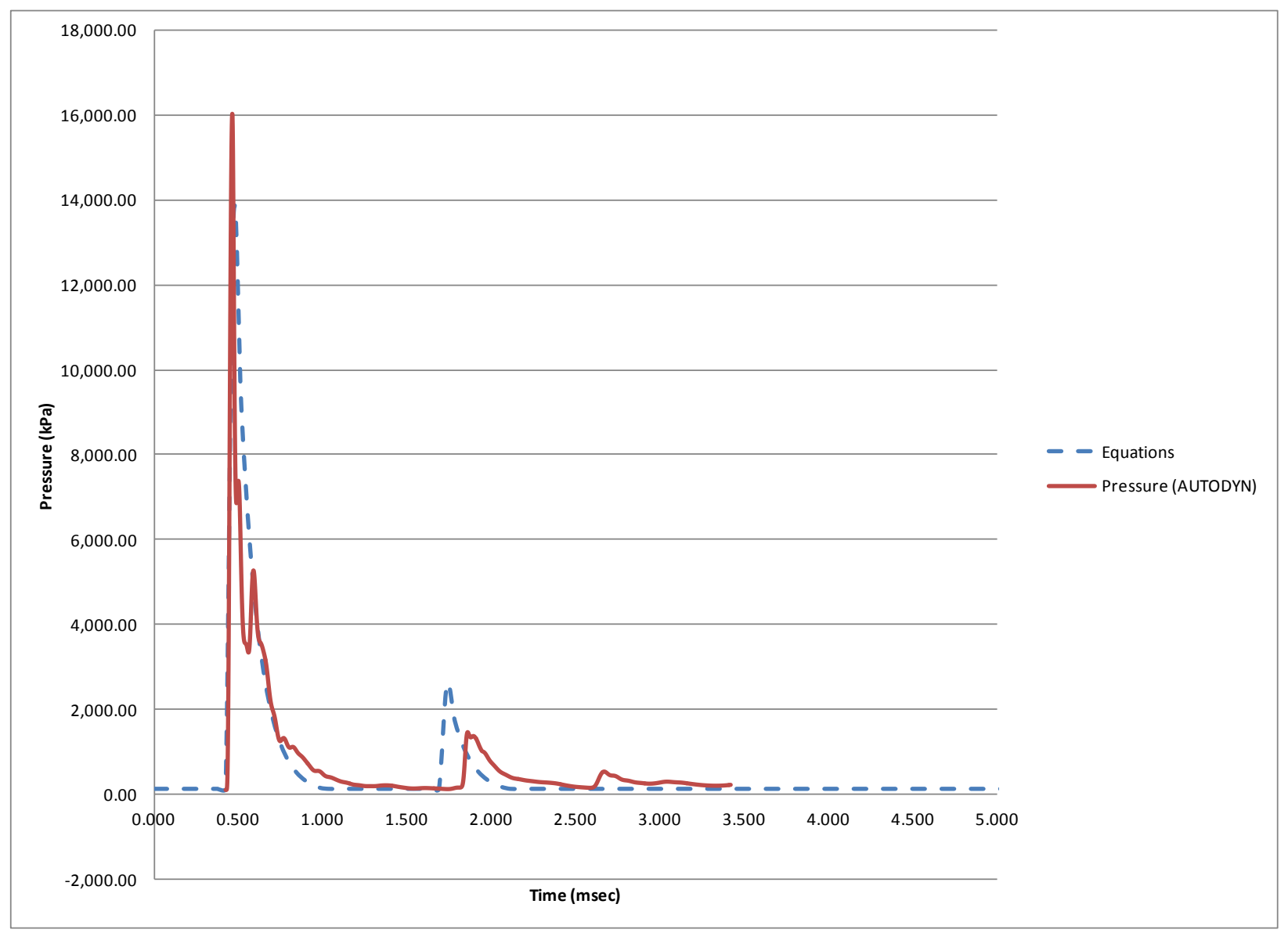

Figure 127: Pressure profile for $5 \mathrm{~kg}$ TNT charge in a $(2.0 \times 2.0)$ container with 0.50 vent area ratio 


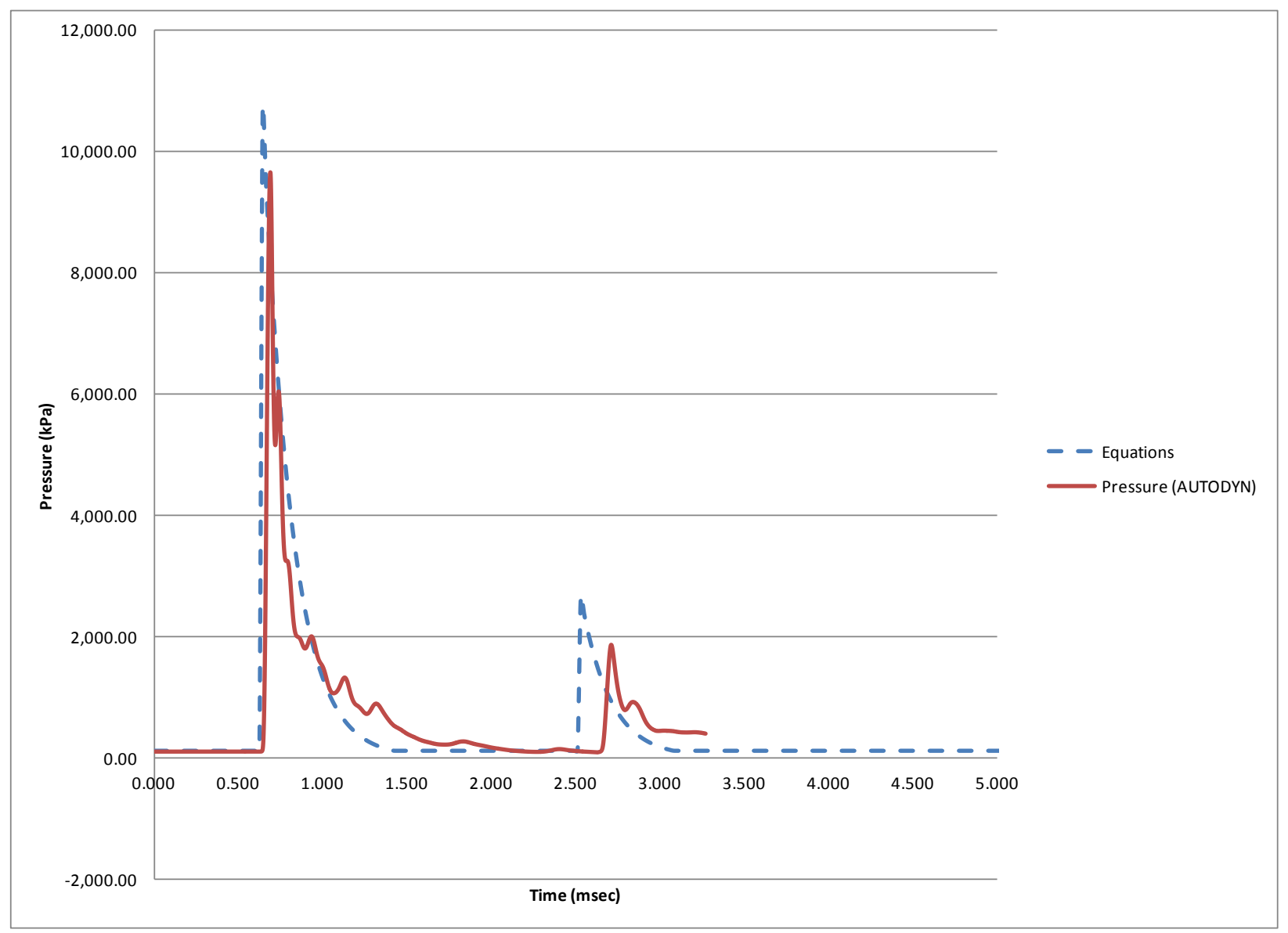

Figure 128: Pressure profile for $5 \mathrm{~kg}$ TNT charge in a $(3.0 \times 3.0)$ container with 0.25 vent area ratio 


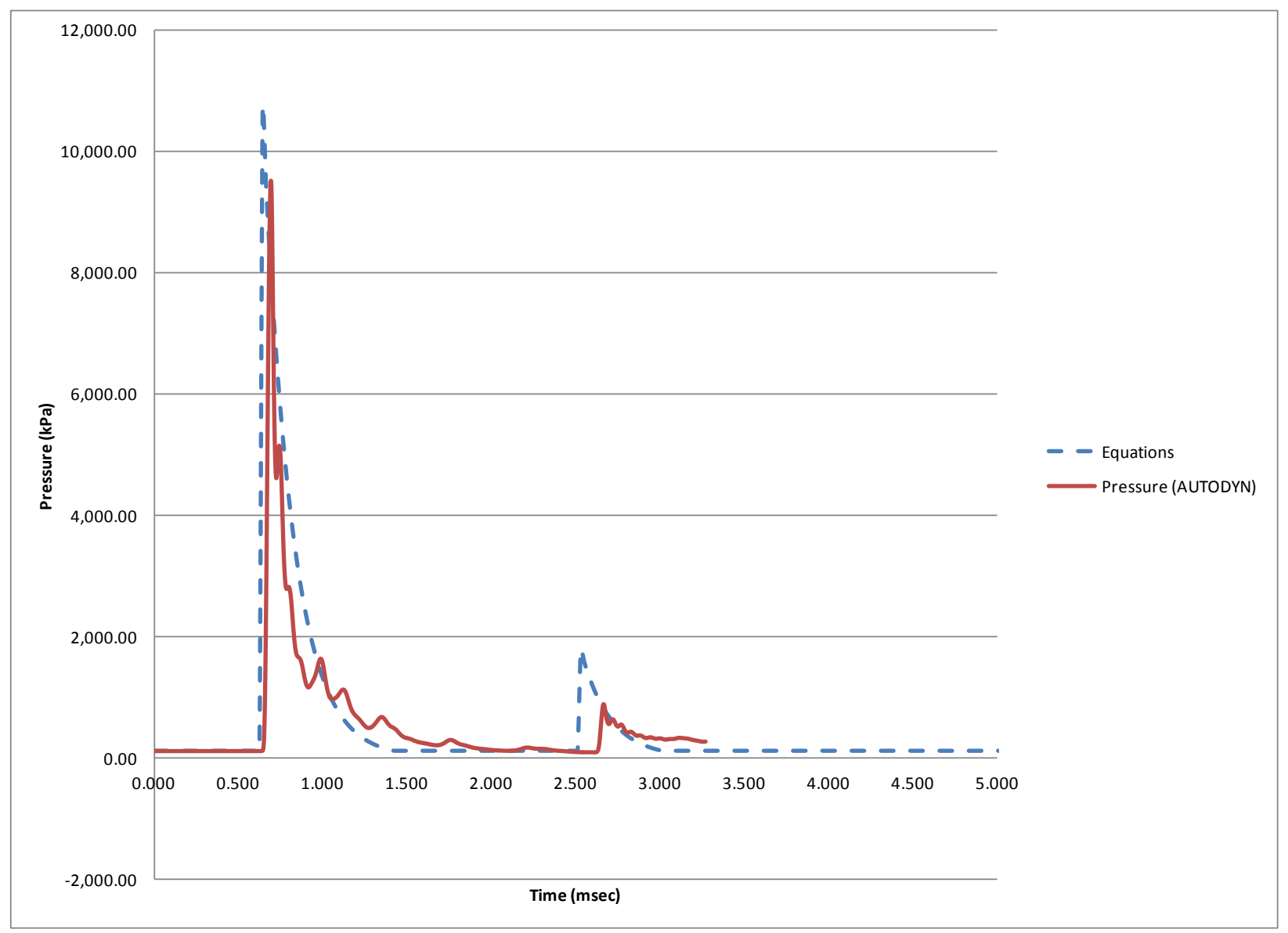

Figure 129: Pressure profile for $5 \mathrm{~kg}$ TNT charge in a $(3.0 \times 3.0)$ container with 0.50 vent area ratio 


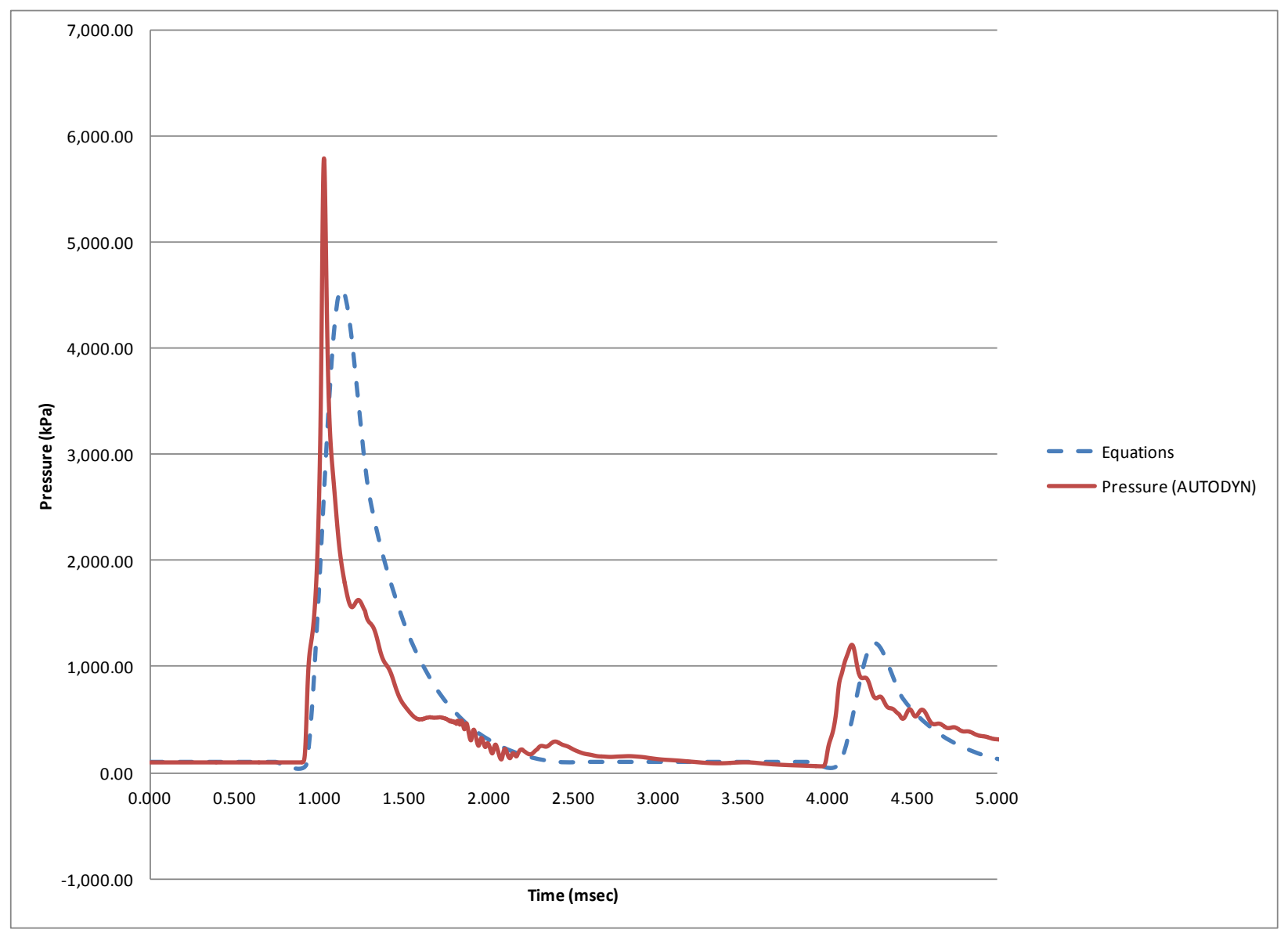

Figure 130: Pressure profile for $5 \mathrm{~kg}$ TNT charge in a $(4.0 \times 4.0)$ container with 0.25 vent area ratio 


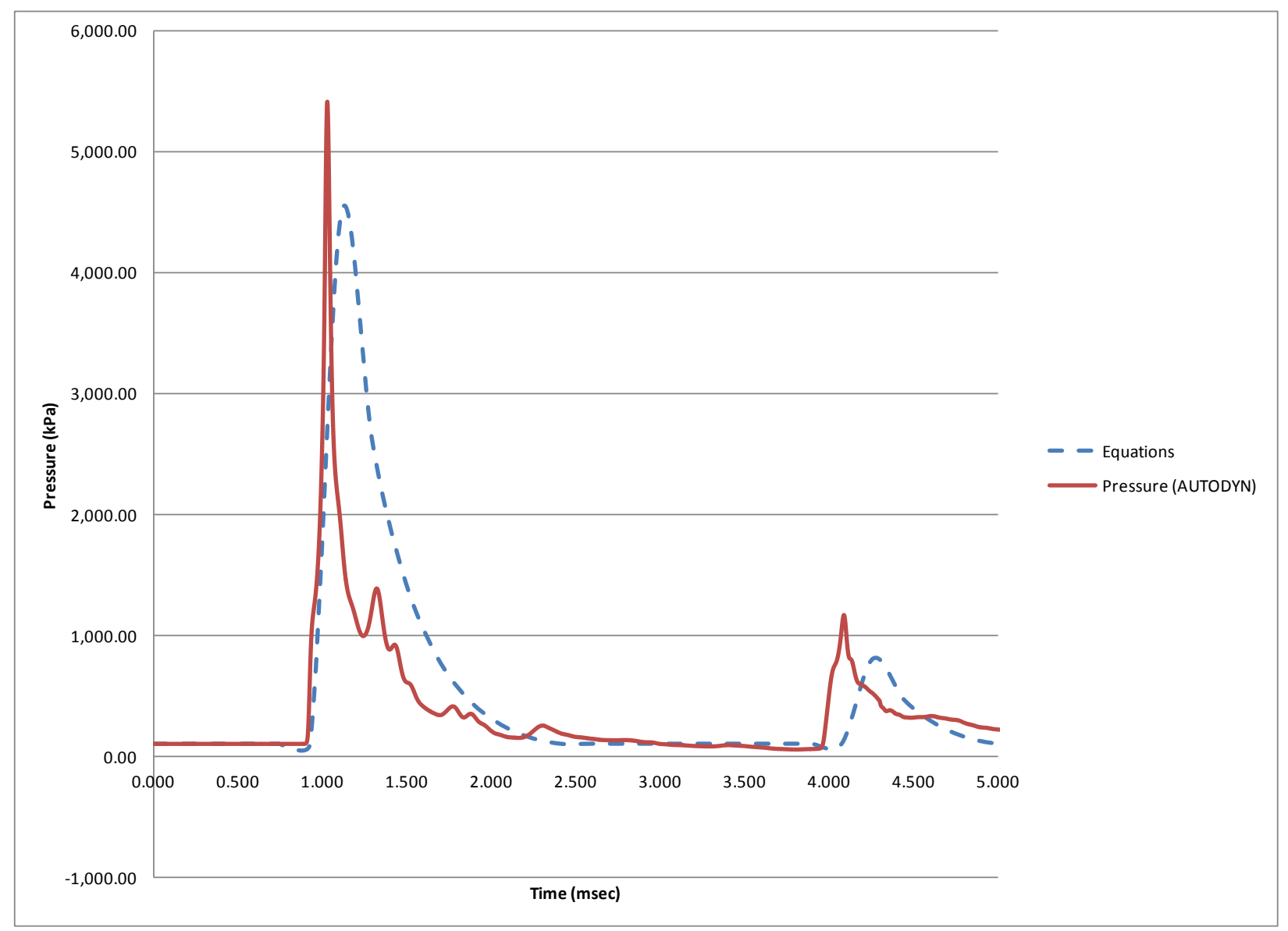

Figure 131: Pressure profile for $5 \mathrm{~kg}$ TNT charge in a $(4.0 \times 4.0)$ container with 0.50 vent area ratio 


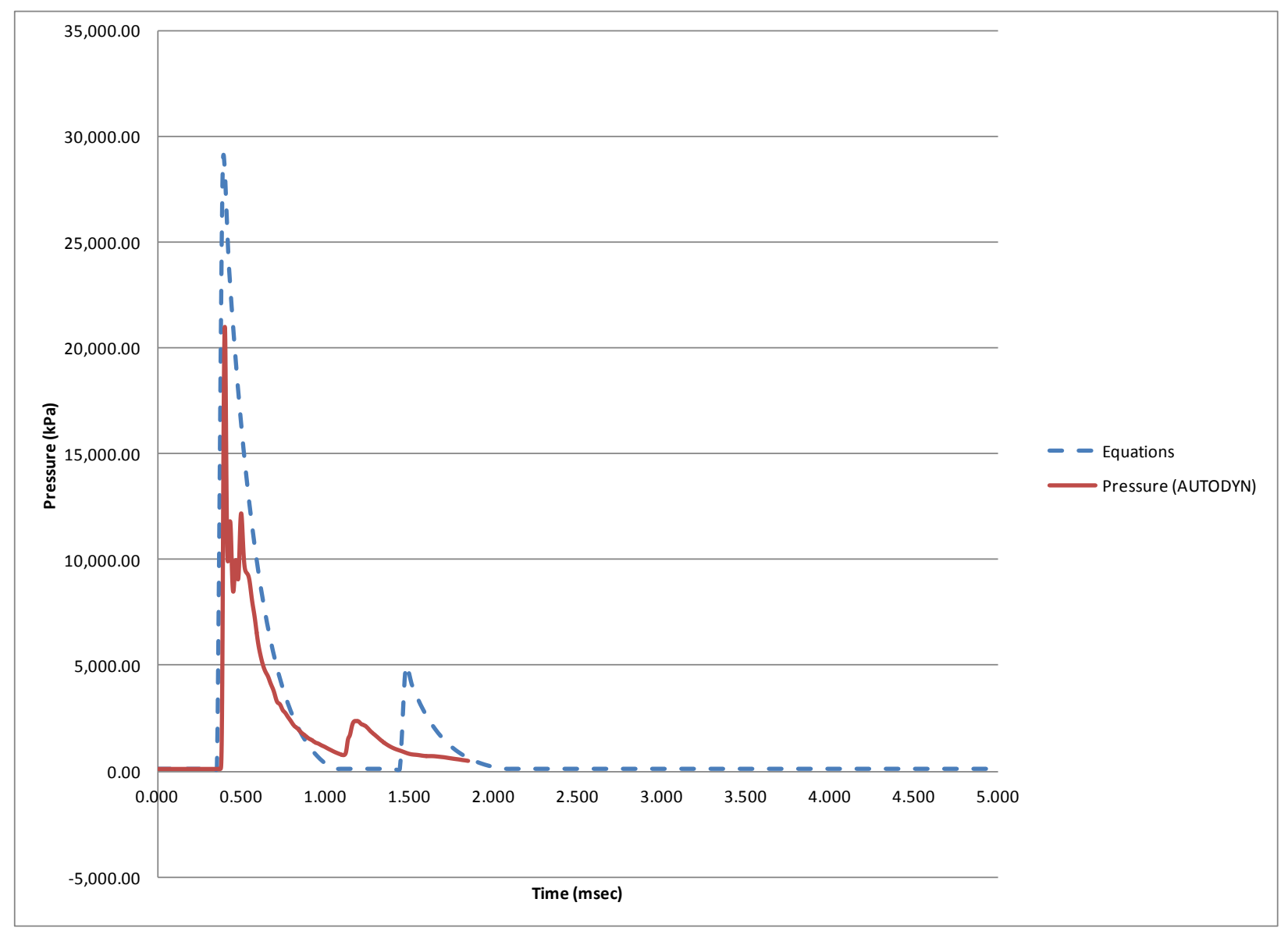

Figure 132: Pressure profile for $10 \mathrm{~kg}$ TNT charge in a $(2.0 \times 2.0)$ container with 0.25 vent area ratio 


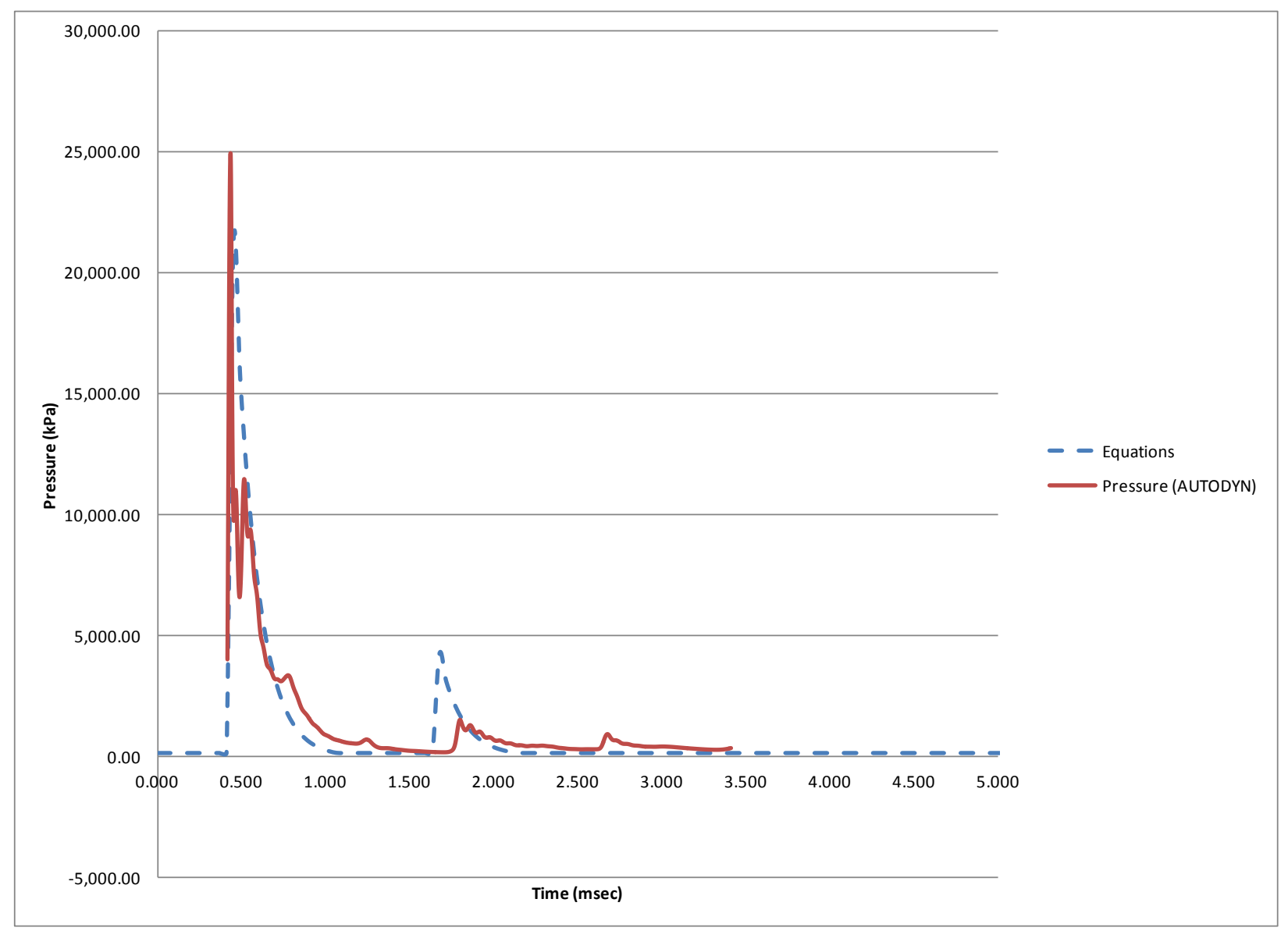

Figure 133: Pressure profile for $10 \mathrm{~kg}$ TNT charge in a $(2.0 \times 2.0)$ container with 0.50 vent area ratio 


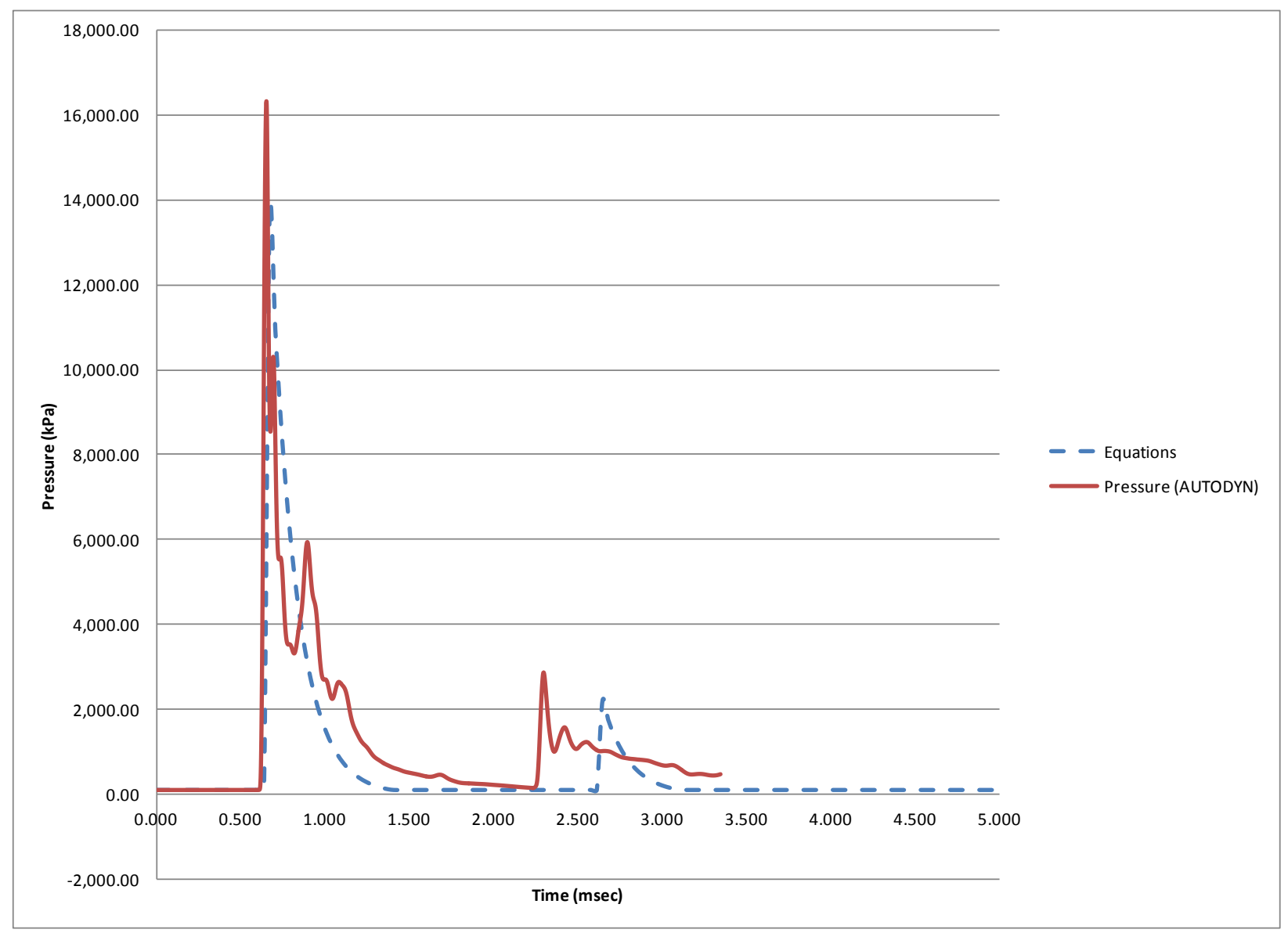

Figure 134: Pressure profile for $10 \mathrm{~kg}$ TNT charge in a $(3.0 \times 3.0)$ container with 0.25 vent area ratio 


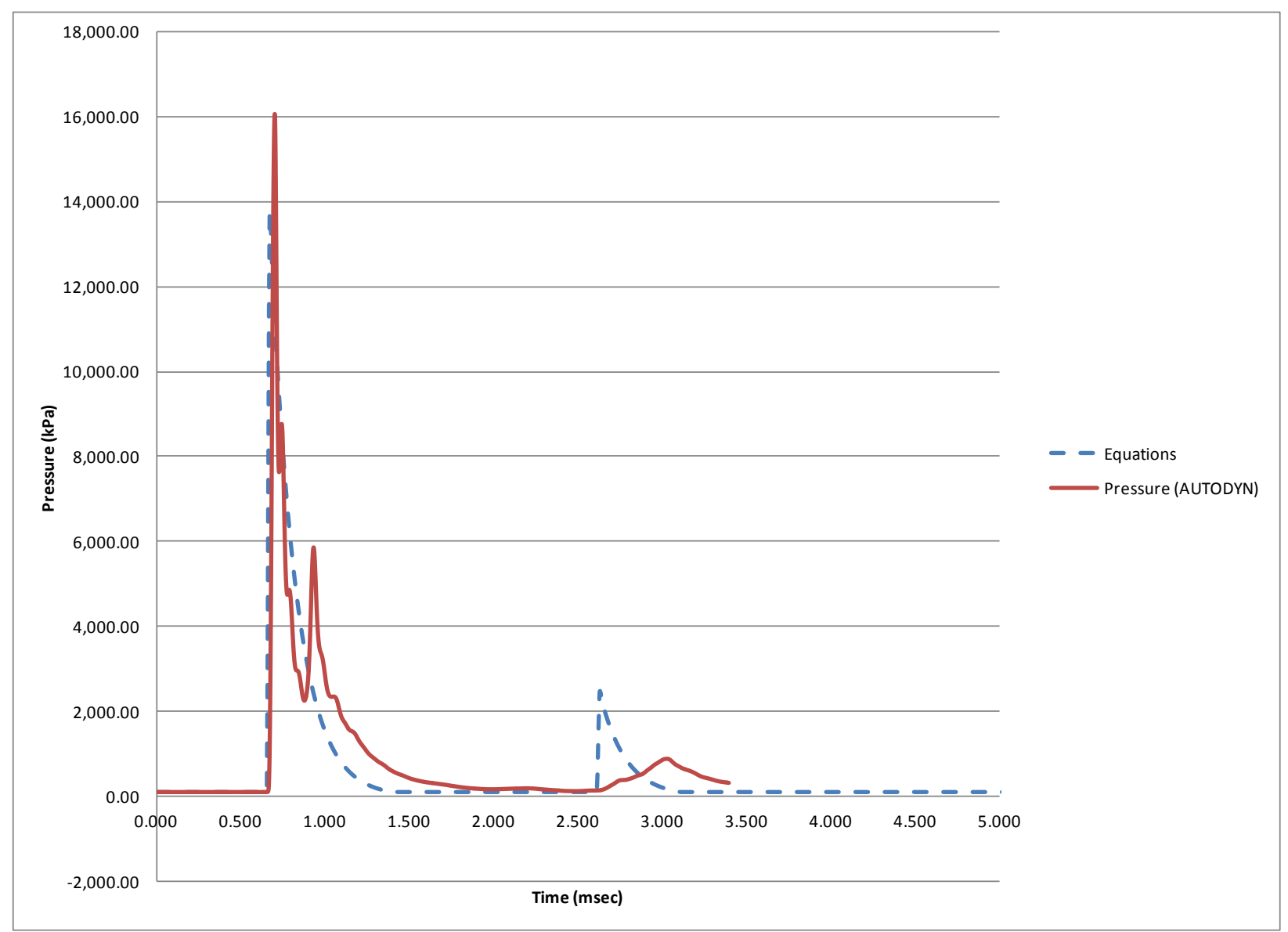

Figure 135: Pressure profile for $10 \mathrm{~kg}$ TNT charge in a $(3.0 \times 3.0)$ container with 0.50 vent area ratio 


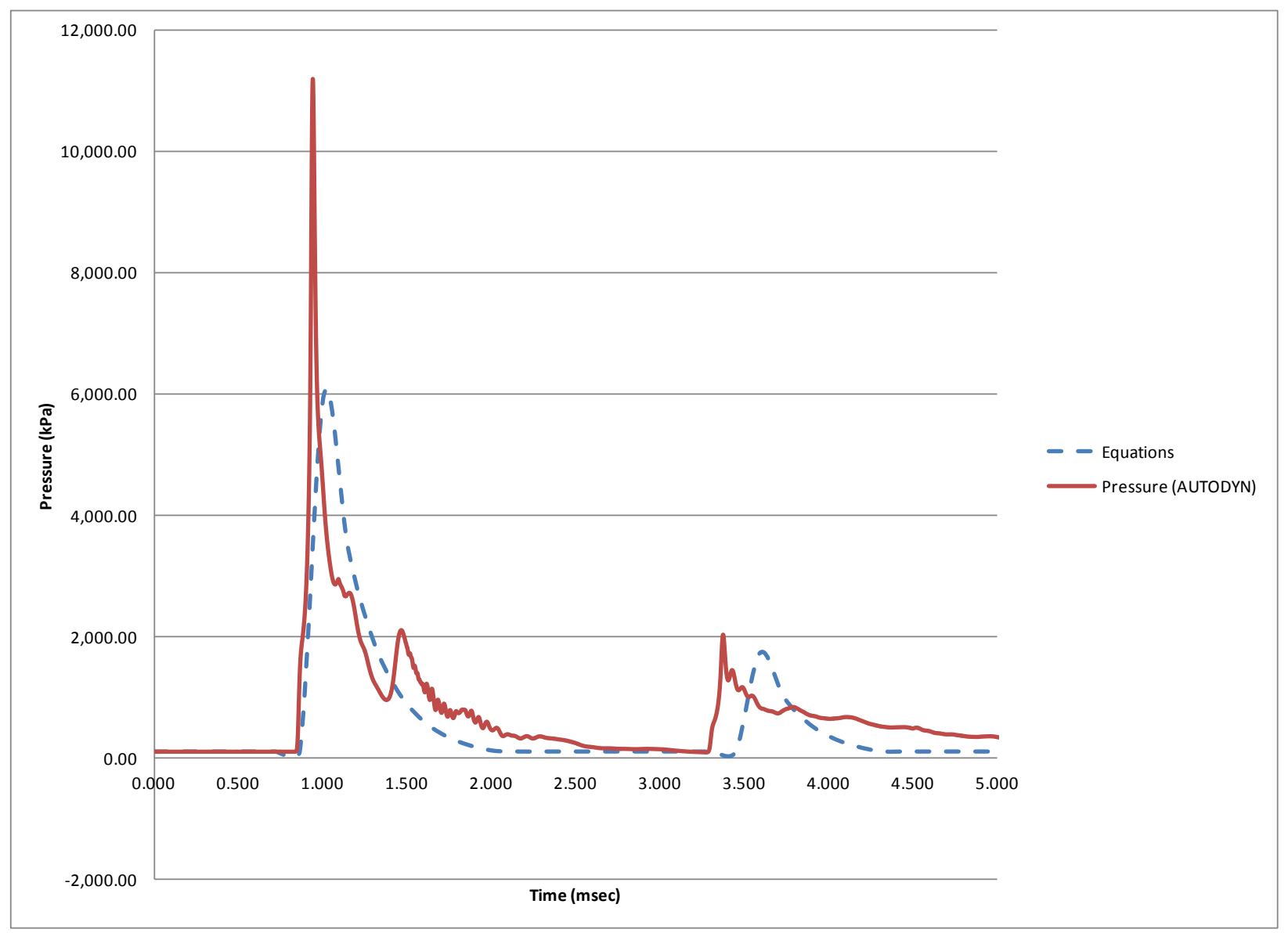

Figure 136: Pressure profile for $10 \mathrm{~kg}$ TNT charge in a $(4.0 \times 4.0)$ container with 0.25 vent area ratio 


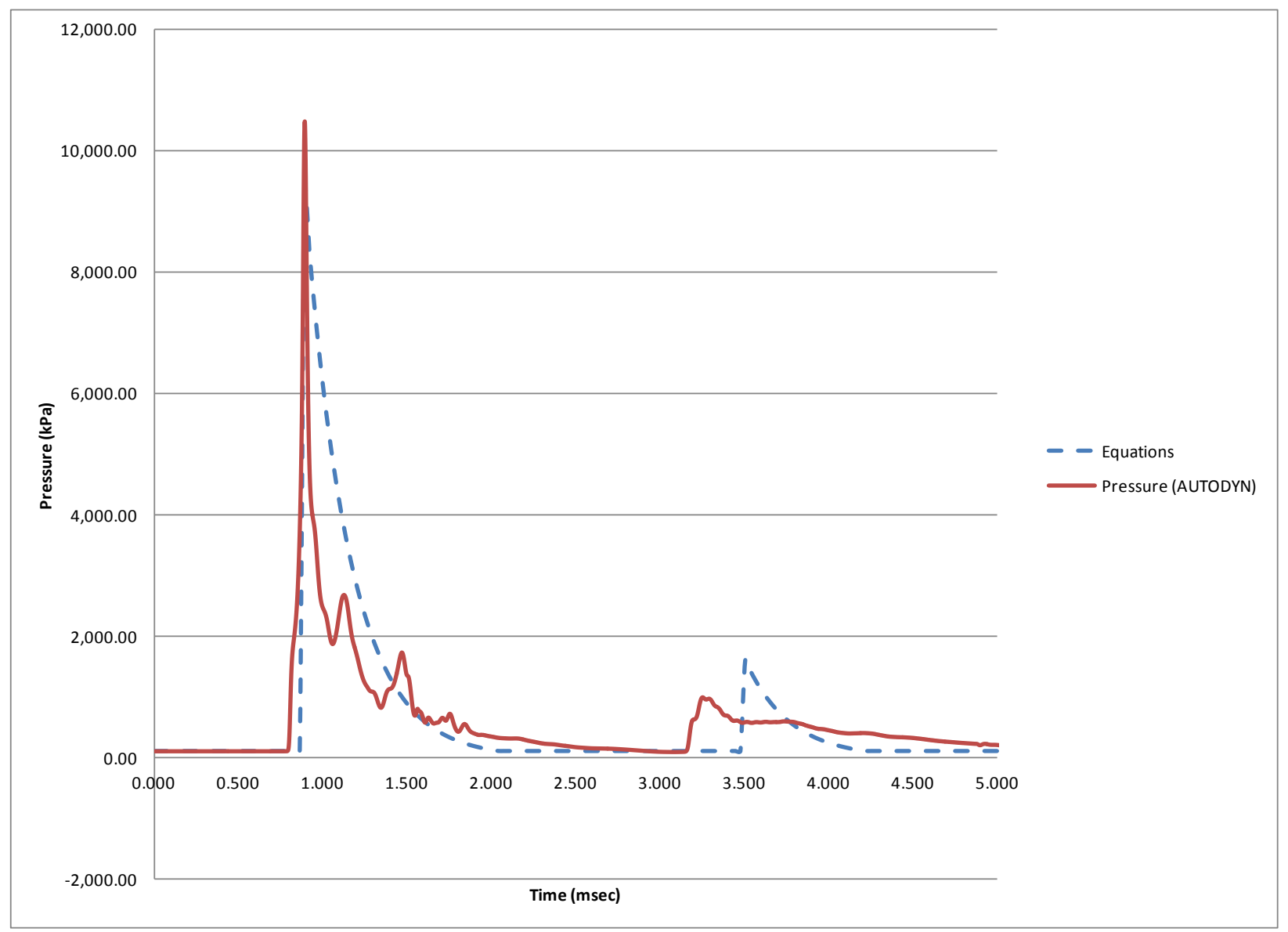

Figure 137: Pressure profile for $10 \mathrm{~kg}$ TNT charge in a $(4.0 \times 4.0)$ container with 0.50 vent area ratio 


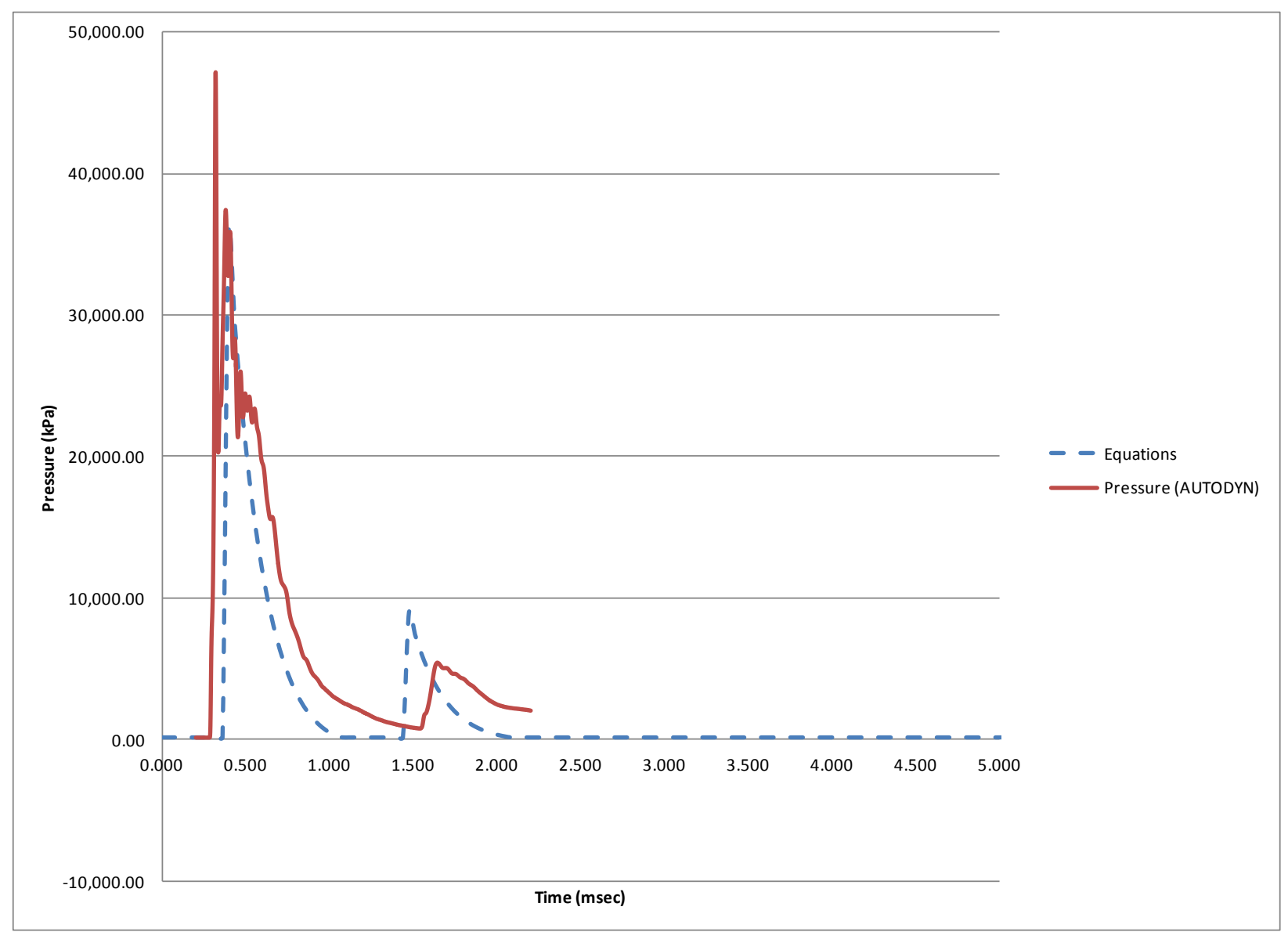

Figure 138: Pressure profile for $20 \mathrm{~kg}$ TNT charge in a $(2.0 \times 2.0)$ container with 0.25 vent area ratio 


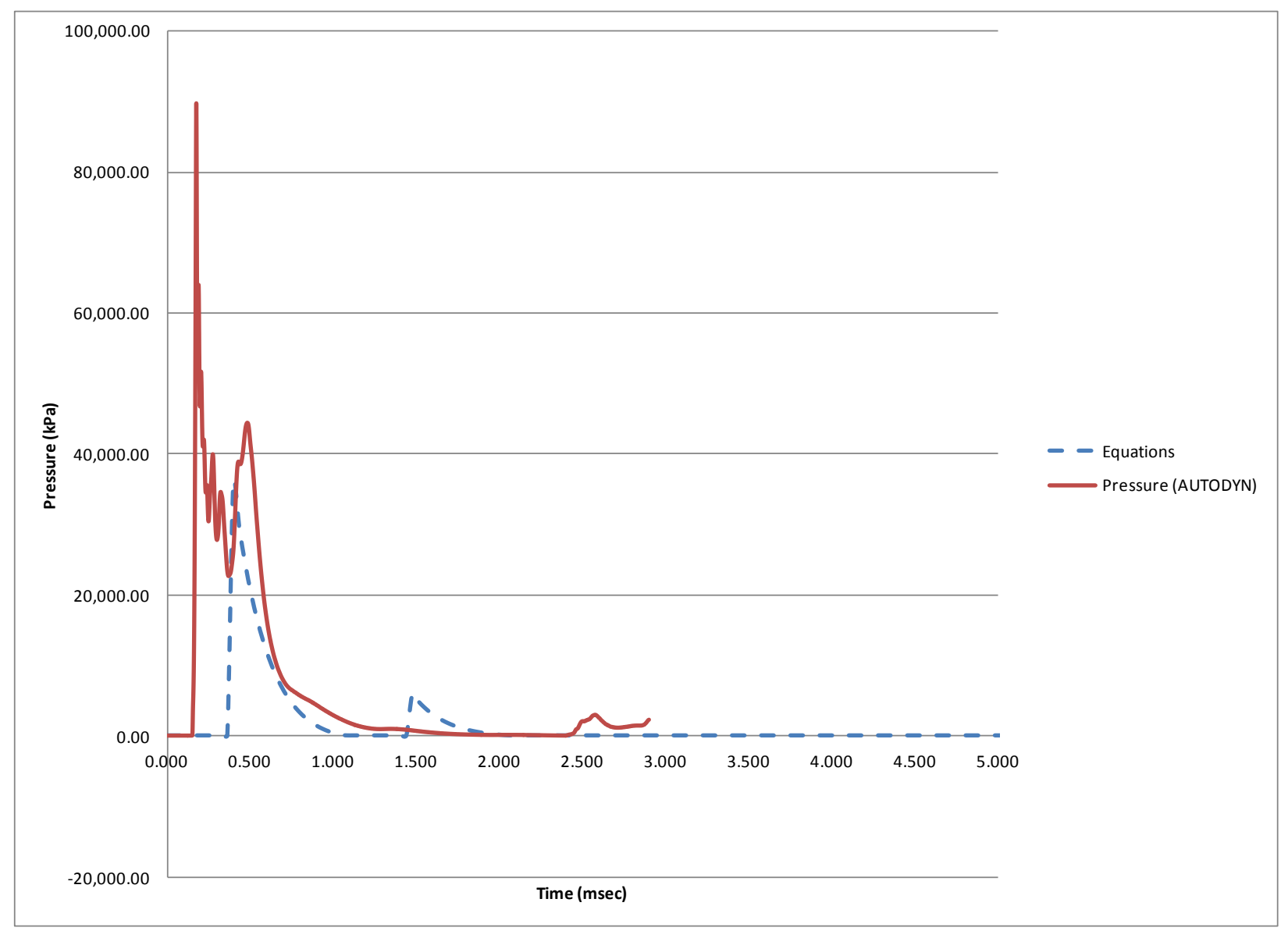

Figure 139: Pressure profile for $20 \mathrm{~kg}$ TNT charge in a $(2.0 \times 2.0)$ container with 0.50 vent area ratio 


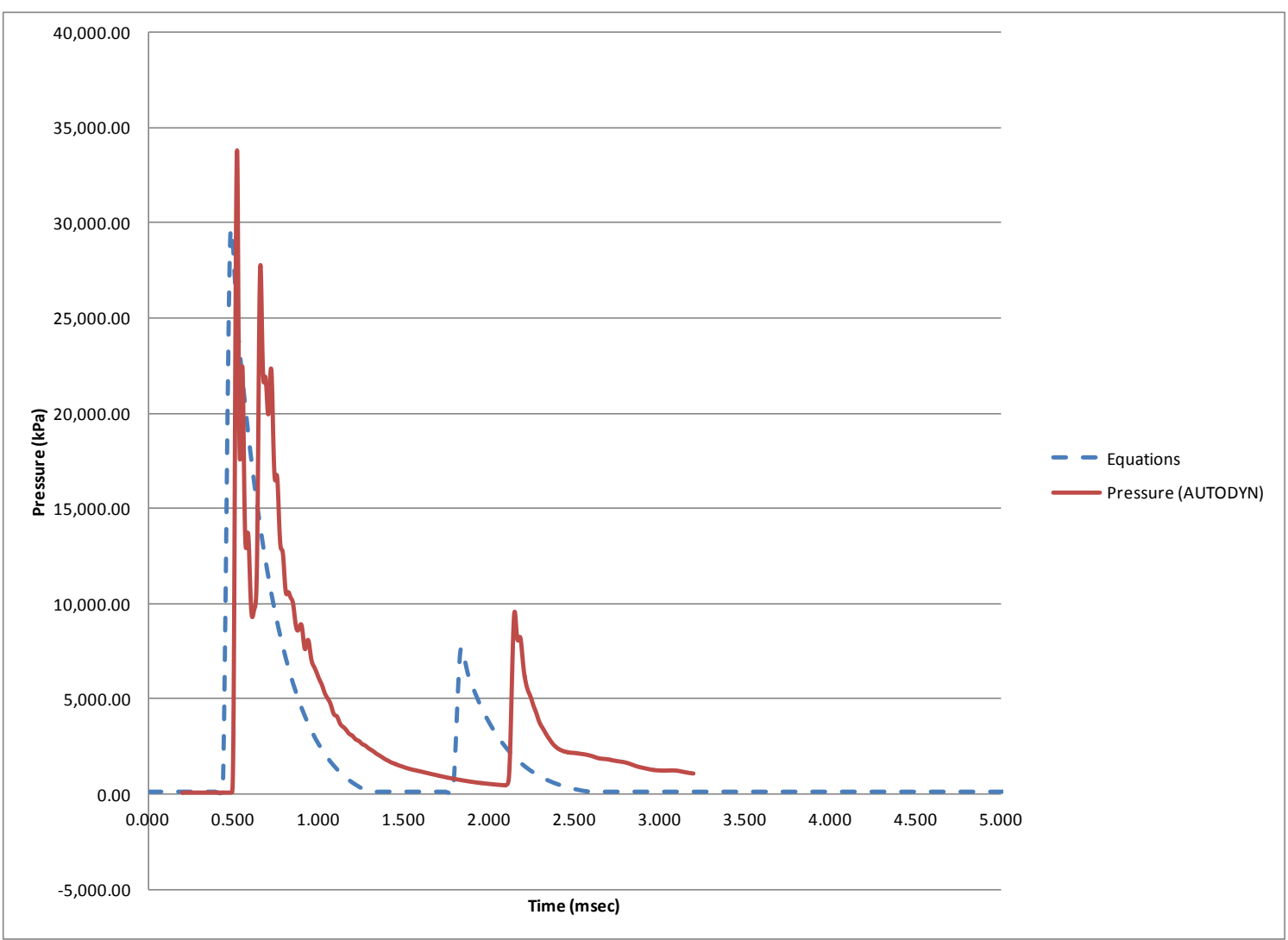

Figure 140: Pressure profile for $20 \mathrm{~kg}$ TNT charge in a $(3.0 \times 3.0)$ container with 0.25 vent area ratio 


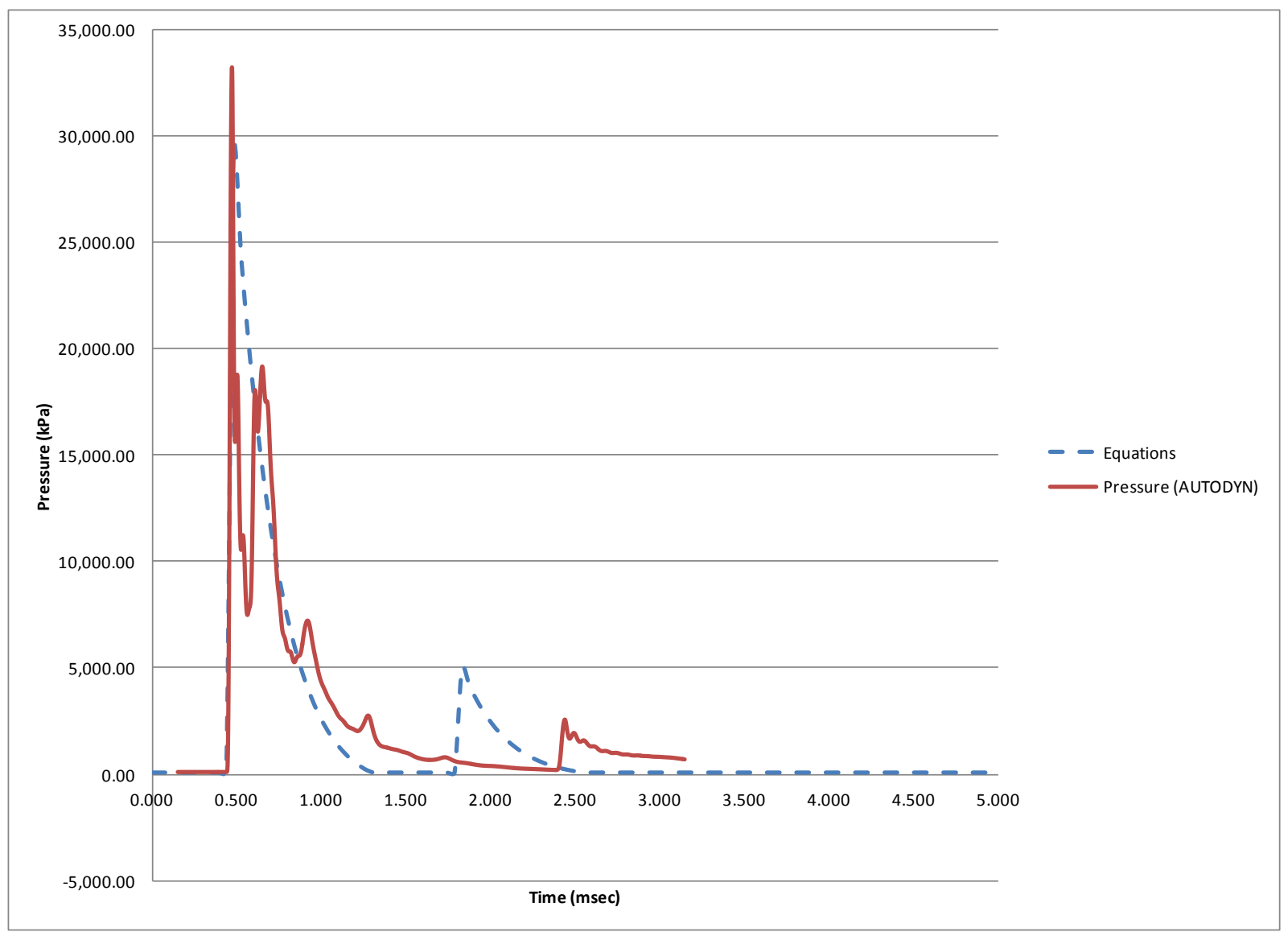

Figure 141: Pressure profile for $20 \mathrm{~kg}$ TNT charge in a $(3.0 \times 3.0)$ container with 0.50 vent area ratio 


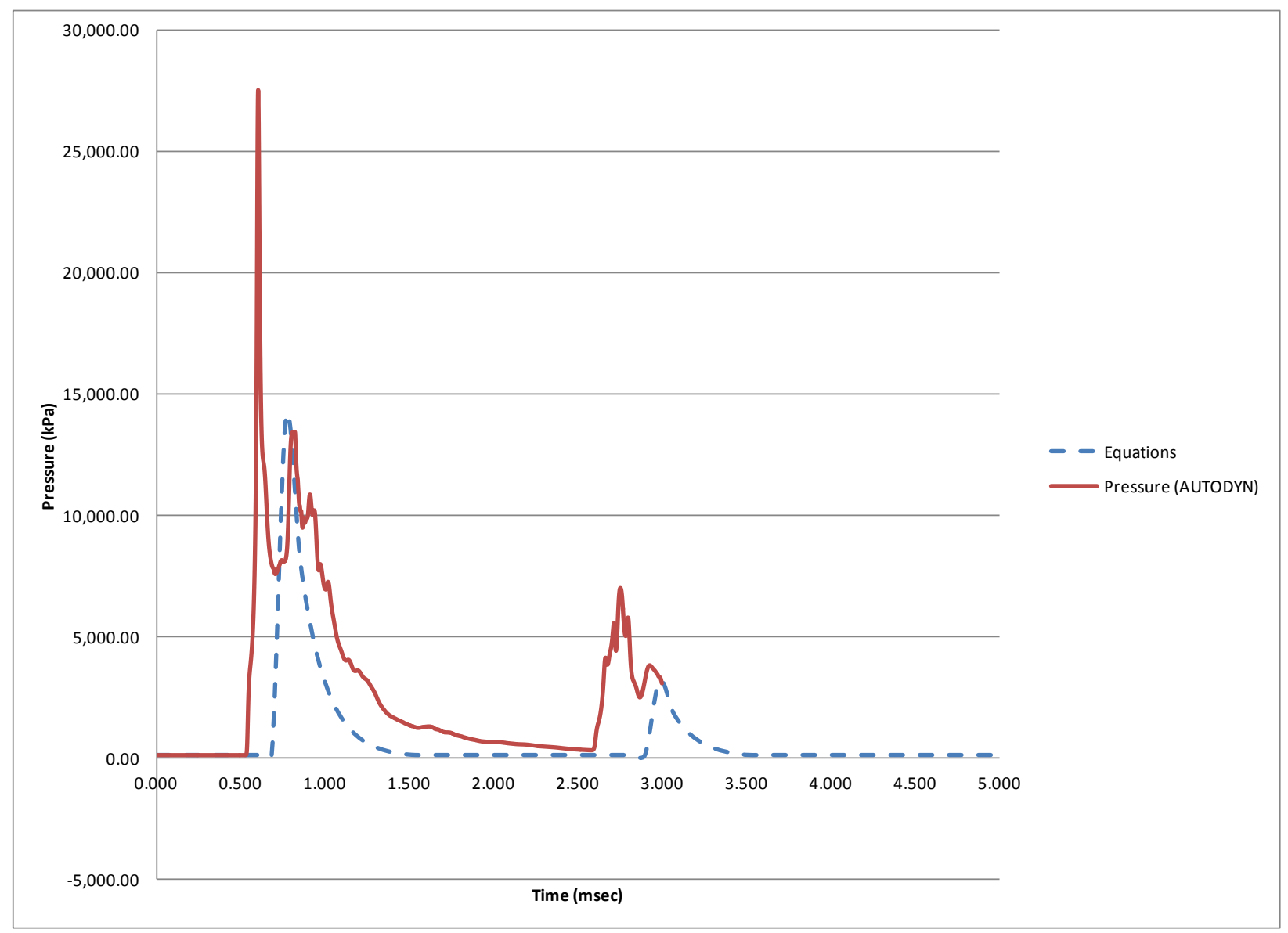

Figure 142: Pressure profile for $20 \mathrm{~kg}$ TNT charge in a $(4.0 \times 4.0)$ container with 0.25 vent area ratio 


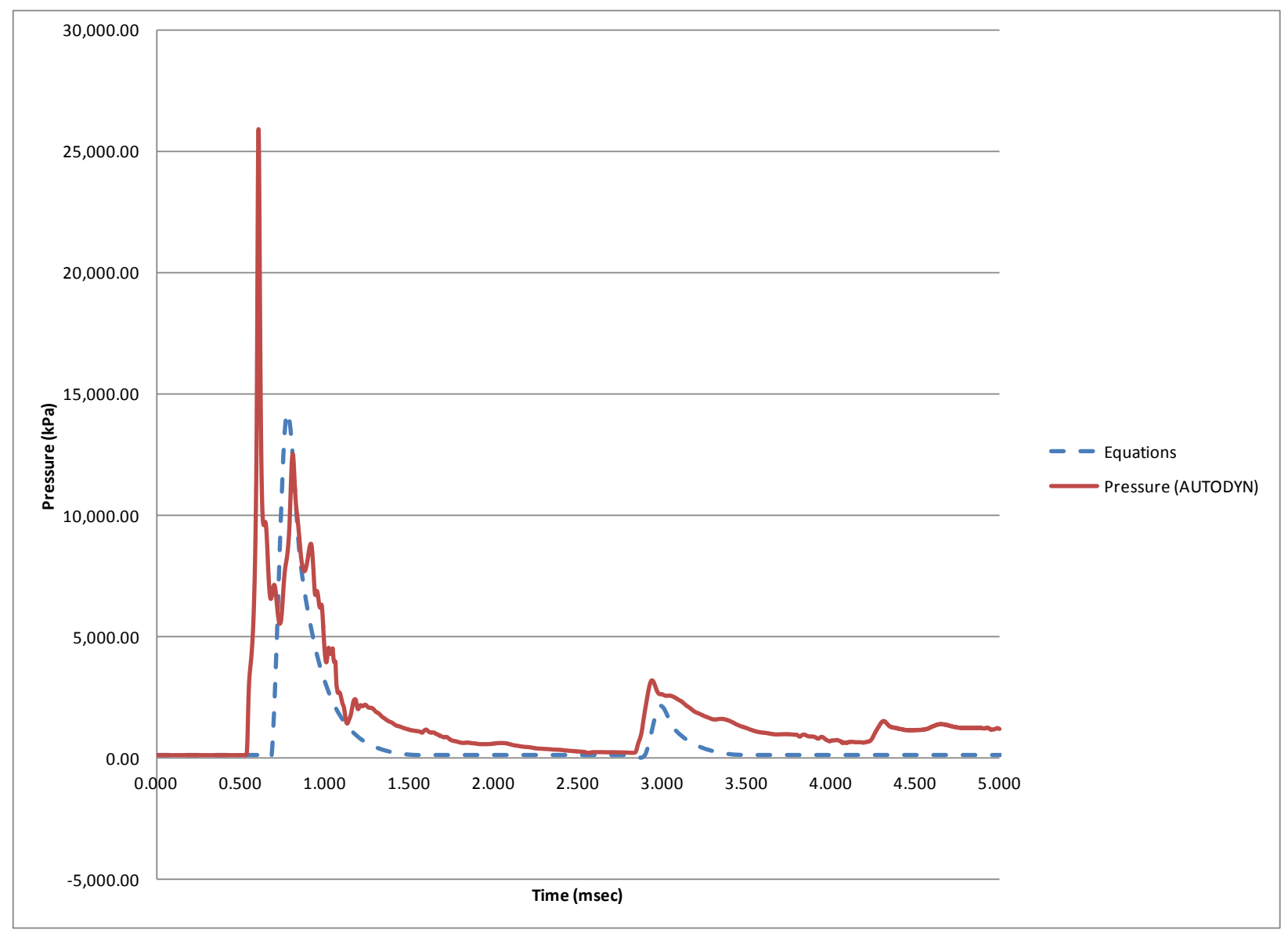

Figure 143: Pressure profile for $20 \mathrm{~kg}$ TNT charge in a $(4.0 \times 4.0)$ container with 0.50 vent area ratio 


\section{Appendix D: Alogarithm for step-by-step solution for elasto-plastic single degree of freedom system}

In this section, a step by step solution for the developed SDOF model is presented.

Compute the properties of the section:

$m$ : Mass of the section $(\mathrm{kg})$

$k$ : Stiffness of the section $(\mathrm{N} / \mathrm{mm})$

$c$ : damping ratio

$R_{t}:$ Resistance in tension $(\mathrm{N})$

$R_{c}:$ Resistance in compression $(\mathrm{N})$

Calculate initial acceleration:

$\ddot{u}_{i}=\frac{P\left(t_{i=0}\right)}{m} \quad\left(\mathrm{~mm} / \mathrm{msec}^{2}\right)$

Calculate initial yield points:

$u_{\max }($ tension $)=\frac{R_{t}}{k} \quad(\mathrm{~mm})$

$u_{\min }($ compression $)=\frac{R_{c}}{k} \quad(\mathrm{~mm})$

For each time step:

Check if the system remains in the previous state of elastic or plastic behaviour using the following code:

Key $=0$ (elastic)

Key $=1$ (Plastic in tension) 
Key $=-1$ (Plastic in compression)

Key $=2$ (Large deformation occur to the element; membrane action takes place)

Key $=3$ (Failure)

Calculate the displacement and the velocity at the mid span of the section. Depending on the deflection and the velocity, the behaviour of the member is defined.
$u_{i}>\Delta_{\max }$
$\mathrm{KEY}=3$
$u_{i}>u_{\max }$ and $v_{i}>0$
KEY=1
$u_{i}>u_{\max }$ and $v_{i}>0$ and membrane
$\mathrm{KEY}=2$
$u_{i}<u_{\min }$ and $v_{i}>0$
$\mathrm{KEY}=-1$
Otherwise
$\mathrm{KEY}=0$

The change in the force applied on the member is explained as below: $\Delta P_{n}^{*}=\Delta P_{n}+m\left(\frac{6}{\Delta t} \dot{u}_{n}+3 \ddot{u}\right)+c\left(3 \dot{u}_{n}+\frac{\Delta t}{2} \ddot{u}\right)$

The stiffness of the system is given as:

$k_{n}^{*}=\left(\frac{6 m}{\Delta t^{2}}+\frac{3 c}{\Delta t}+k\right)$

where the stiffness $(\mathrm{k})$ is given by the following equations:
If $\mathrm{KEY}=0$,
$k_{i}=k_{e}$
If $\mathrm{KEY}=1$,
$k_{i}=0$
If $\mathrm{KEY}=-1$,
$k_{i}=0$ 
If $\mathrm{KEY}=2$,

$$
k_{i}=\frac{R_{i}}{u_{i}}
$$

The change in displacement is given as:

$$
\Delta u_{n}=\frac{\Delta p_{n}{ }^{*}}{k_{n}{ }^{*}}
$$

The change in velocity is given as:

$$
\Delta \dot{u}=\frac{3}{\Delta t} \Delta u_{n}-3 \dot{u_{n}} \frac{\Delta t}{2} \ddot{u}_{n}
$$

The acceleration of the mid span deformation is calculated at the end of each time interval using the dynamic equation of equilibrium

$$
\ddot{u}_{i+1}=\frac{1}{m}\left[P\left(t_{i+1}\right)-c_{i+1} \dot{u}_{i+1}-R\right]
$$

at which the value of $\mathrm{R}$ depends on the behaviour of the steel plate at a given time step. The following equations are used to calculate the resistance $\left(R_{i}\right)$
If $\mathrm{KEY}=3$,$$
R=0
$$
If $\mathrm{KEY}=0$,

$$
R_{i}=R_{t}-\left(u_{\max }-u_{i}\right) * k_{i}
$$
If $\mathrm{KEY}=1$,
$R_{i}=R_{t}$
If $\mathrm{KEY}=-1$,

$$
R_{i}=-\left(R_{t}\right)
$$
If $\mathrm{KEY}=2$,

$$
R_{i}=\left(\left(\frac{u-t}{t}\right) * R_{t}\right)+R_{t}
$$

Calculate the elongation in the steel member due to the tension force due to large deformation: 
$\Delta_{\text {elongation }}=\frac{L}{2}(\cos \theta-1)$

Calculate the tension force in the member:

$$
T=\frac{\Delta_{\text {elongation }}{ }^{*} E^{*} A}{L}+\frac{M}{t / 2}
$$

where $\mathrm{M}$ is the moment at end supports assuming an equivalent static load on the steel section. The equivalent static load and end moment are calculated using the following equations:

$$
\begin{aligned}
& M_{u l t}=\frac{r_{u} * L^{2}}{12} \\
& r_{u}=\frac{384 \Delta_{e} * E * I}{5 L^{4}}
\end{aligned}
$$

